\title{
The use of personal development plans : effects, purposes and supporting conditions
}

Citation for published version (APA):

Beausaert, S. A. J. (2011). The use of personal development plans : effects, purposes and supporting conditions. [Doctoral Thesis, Maastricht University]. Universitaire Pers Maastricht. https://doi.org/10.26481/dis.20111019sb

Document status and date:

Published: 01/01/2011

DOI:

10.26481/dis.20111019sb

Document Version:

Publisher's PDF, also known as Version of record

\section{Please check the document version of this publication:}

- A submitted manuscript is the version of the article upon submission and before peer-review. There can be important differences between the submitted version and the official published version of record. People interested in the research are advised to contact the author for the final version of the publication, or visit the DOI to the publisher's website.

- The final author version and the galley proof are versions of the publication after peer review.

- The final published version features the final layout of the paper including the volume, issue and page numbers.

Link to publication

\footnotetext{
General rights rights.

- You may freely distribute the URL identifying the publication in the public portal. please follow below link for the End User Agreement:

www.umlib.nl/taverne-license

Take down policy

If you believe that this document breaches copyright please contact us at:

repository@maastrichtuniversity.nl

providing details and we will investigate your claim.
}

Copyright and moral rights for the publications made accessible in the public portal are retained by the authors and/or other copyright owners and it is a condition of accessing publications that users recognise and abide by the legal requirements associated with these

- Users may download and print one copy of any publication from the public portal for the purpose of private study or research.

- You may not further distribute the material or use it for any profit-making activity or commercial gain

If the publication is distributed under the terms of Article $25 \mathrm{fa}$ of the Dutch Copyright Act, indicated by the "Taverne" license above, 
The use of

Personal Development Plans

in the workplace

Effects, purposes and supporting conditions

Simon A.J. Beausaert 


\section{ico}

(C) Simon A.J. Beausaert, Maastricht, The Netherlands, 2011

ISBN: 9789461590749

Cover: Jo Frenken, Jan Van Eyck Academy

All rights reserved

Printed and bound by Datawyse, Maastricht, The Netherlands 


\section{The use of}

\section{Personal Development Plans}

in the workplace

\section{Effects, purposes and supporting conditions}

Proefschrift

Ter verkrijging van de graad van doctor

aan de Universiteit Maastricht

op gezag van de rector magnificus

prof. mr. G. P. M. F. Mols

volgens het besluit van het College van Decanen

in het openbaar te verdedigen

op woensdag 19 oktober 2011 om 16:00 uur

door

Simon A.J. Beausaert

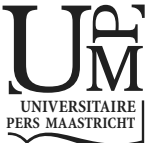




\section{Promotores}

Prof. dr. M. S. R. Segers

Prof. dr. W. H. Gijselaers

\section{Beoordelingscommissie}

Prof. dr. A. De Grip (chair)

Dr. P. van den Bossche, University of Antwerp/Maastricht University

Prof. dr. R. Poell, University of Tilburg

Prof. dr. K. Smith, University of Bergen, Norway

Prof. dr. C. van der Vleuten 


\section{Acknowledgements}

This book that contributed to my learning process and personal development as a researcher and teacher can be seen as a kind of personal development plan which was supported by many inspiring mentors and coaches. I would like to thank everyone that contributed to this book in his or her way.

Although I find it hard to express my gratitude in words, I would like to dedicate a special thank you to:

Henny Dankers, for her logistical support, for listening and caring, for being my second mother;

Jeannette Hommes, for always being interested in my research, for helping me with finding companies who were willing to participate in my research, for giving the trainings to the supervisors, for mentioning relevant articles;

Piet van den Bossche, for providing me with a survival guide 'A Belgian living and working in Maastricht', for the very inspiring discussions, for the tips and tricks;

Janine van der Rijt, for her friendship and moral support, for the exchange of useful ideas, and for the tips and tricks. How was NY? Hilarious;

Bart Rienties, for being my roommate through the good and the bad times, for listening, for taking care of sufficient breaks and drinks, for involving me in various of your projects;

Amber Dailey, for being my other roommate through the good and te bad times, for helping me finding the right English words, for listing when you felt I needed it the most, for the wonderful cup;

Jan Nijhuis, for the many discussions on statistical issues, for informing me on relevant evolutions in the Netherlands, for the numerous one-liners during lunch;

Bas Giesbers, Danielle Townsend-Prevoo, Diana Baas, Paul Jacobs, Catherine Gabelica, Katerina Bohle Carbonell, Maike Gerken, Alexandra Niculescu, Anna Chatzimichali, Ellen Nelissen, Gwen Noteborn, Tamerius Cohen, Beverly Wilson-Wunsch, Martin Rehm, Wilfred van Dellen, Oscar van den Wijngaard, Shirley Thomas, Sonja Zaar, Nga Pham, Rinske Franssen, and Laura Pirani, for their friendship, support, reflective discussions;

Didier Fouarge, for showing me how to think as an economist, for our fruitful discussion related to the study discussed in Chapter 3;

My thesis students, for their inspiring ideas and interesting discussions;

Kari Smith and the colleagues from the University of Bergen: Kari K., Vibeke, Ketil, Marit, Ingrid, Kari B., John... for offering me a warm and quiet environment to write, for listening, for their friendship, for making my stay in Bergen that pleasant and unforgettable; 
The organizations I worked with, their HRM managers and the employees who participated in the research. A special thank you goes to Ton Claessen, Maud Vossen, Wil Vink, Joyce Heijne and Ilona Kacanic from GITP;

Tijs Boussier, for proof-reading this book;

Jo Frenken, for designing the wonderful cover and Petra van der Jeught, for introducing me to Jo;

Wim Gijselaers, my second supervisor, for his critical view and devoted feedback on my writings, for pushing me to seek my own limits, for providing me with the resources I needed;

Mien Segers, my supervisor, for being a coach and friend, for her involvement, for the inspiring brainstorms, for her expertise and management skills, and preparedness to solve all my questions, at any time, for helping this project succeed. Mien, I owe you a very big thank you;

My friends and my "train-buddies", for detracting me and pretending they were really interested in what I was studying;

My family and in particular my grandmother and grandfather, thank you for always believing in me and surrounding me with your love;

My partner, for being my everything, for loving and carrying me, for being there anyplace, anywhere, anytime, for detracting me, for coping with me in times of stress and hard work, for mentioning once in a while that it is 'just a research' (isn't it?), for helping me with the lay-out;

My mum and dad - although I realize that words can not express what you mean and meant to me, and although I know I do not mention or show this enough, I will still give it a try - for their love and caring, for being interested, for creating the opportunities and the chances that made me the person I am today, thank you! 


\section{Table of Content}

\section{CHAPTER 1 INTRODUCTION}

1. Continuing professional development and PDPs: Practice and theory development

2. A Personal Development Plan (PDP): Definition ..............................................18

3. Overview of the dissertation and research questions ..................................20

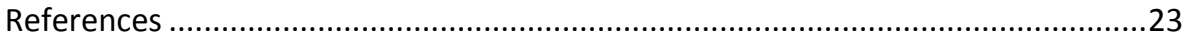

\section{CHAPTER 2 THE USE OF PERSONAL DEVELOPMENT PLANS IN THE WORKPLACE: A LITERATURE REVIEW}

Based upon Beausaert, S., Segers, M., van der Rijt, J., Gijselaers, W. (2011). The use of Personal Development Plans in the workplace: A literature review. In P. van den Bossche, W. Gijselaers, \& R. Milter (Eds.), Building learning experiences in a changing world, Advances in Business Education and Training III (pp. 235-265). Dordrecht: Springer.

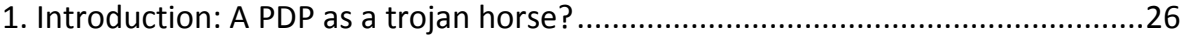

1.1. A Personal Development Plan: Toward a definition ...................................26

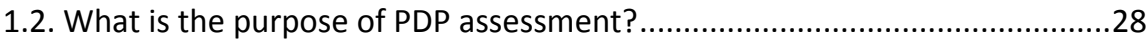

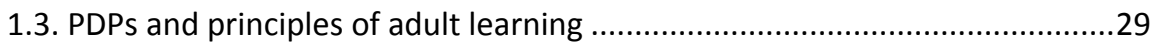

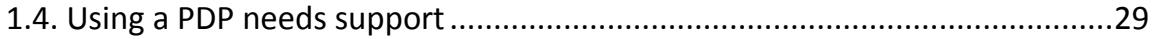

1.5. The goal of this literature review .......................................................... 30

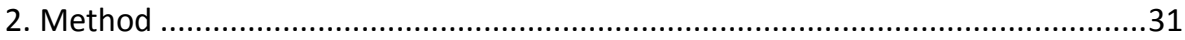

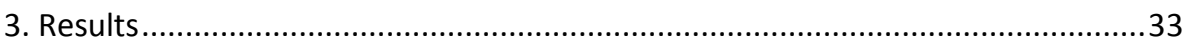

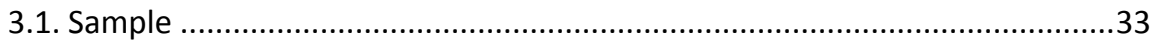

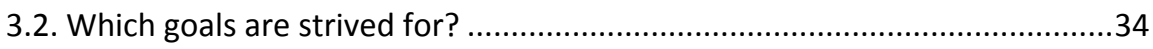

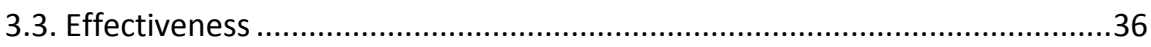

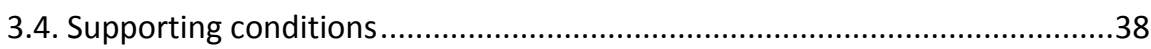

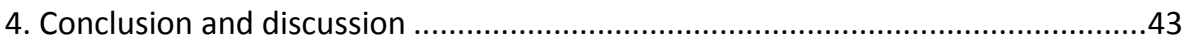

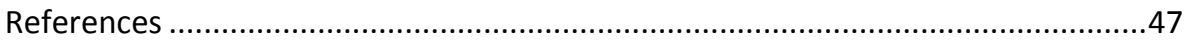

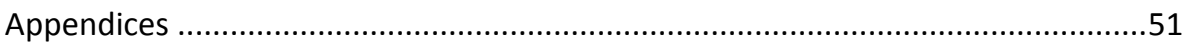

CHAPTER 3 THE EFFECT OF USING A PERSONAL DEVELOPMENT PLAN (PDP) ON THE PHARMACY ASSISTANT'S LEARNING AND PERFORMANCE

Based upon Beausaert, S., Segers, M., Fouarge, D., \& Gijselaers, W. (submitted). The effect of using a Personal Development Plan (PDP) on the pharmacy assistant's learning and performance. 


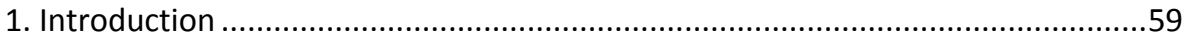

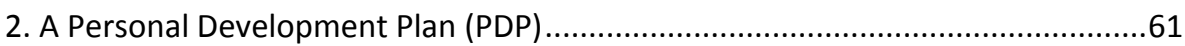

3. The effectiveness of PDPs: Previous research and hypotheses .............................62

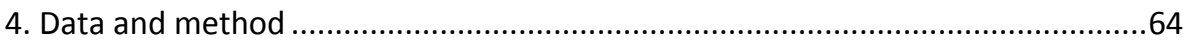

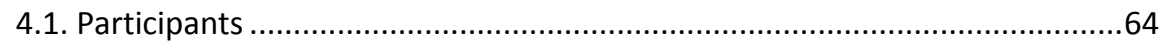

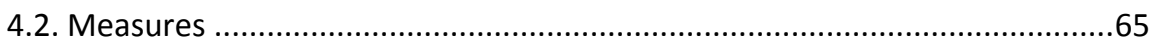

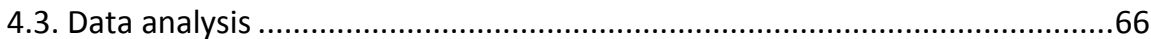

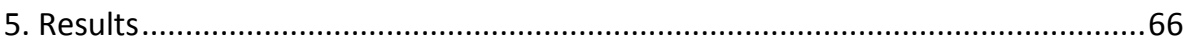

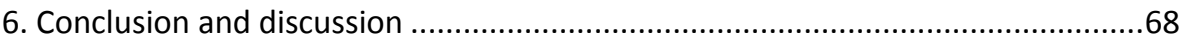

6.1. Implications for future research .........................................................

6.2. Implications for practice .......................................................................71

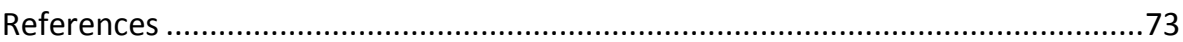

CHAPTER 4 USING A PDP FOR DIFFERENT PURPOSES: ITS INFLUENCE ON UNDERTAKING LEARNING ACTIVITES AND JOB PERFORMANCE

Based upon Beausaert, S., Segers, M., \& Gijselaers, W. (2011). Using a Personal Development Plan for different purposes: Its influence on undertaking learning activities and job performance. Vocations and Learning.

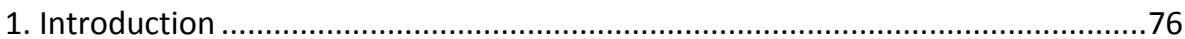

1.1. A Personal Development Plan (PDP): What? ...............................................78

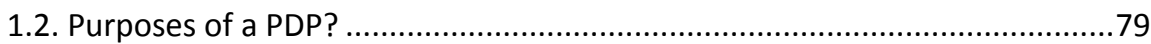

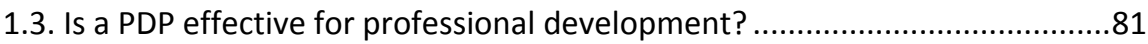

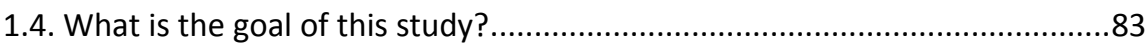

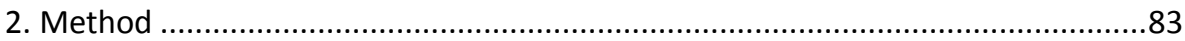

2.1. The participating organizations and their employees...................................83

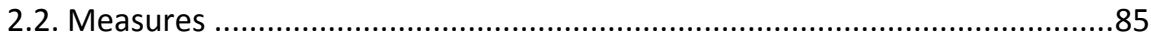

2.3. Procedure

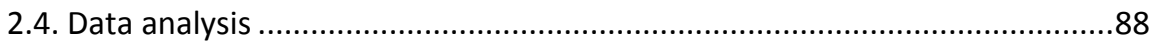

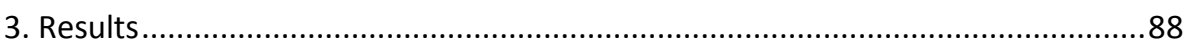

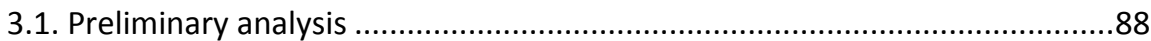

3.2. Hypothesis 1: Perceiving the PDP as a learning and development tool will predict the employee's undertaking of learning activities and the employee's performance significantly positively

3.3. Hypothesis 2: Perceiving the PDP as a selection and certification tool will predict the employee's undertaking of learning activities and the employee's performance significantly negatively.

3.4. Hypothesis 3: In the case both learning/development and promotion/ selection purposes are taken into account, perceiving the PDP as a learning and development tool will be the most powerful predictor of the employee's undertaking of learning activities 
3.5. ANOVA: Differences between organizations ............................................93

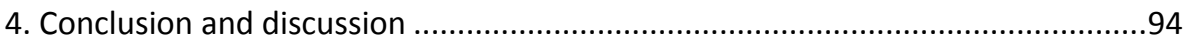

4.1. Limitations and future research ............................................................96

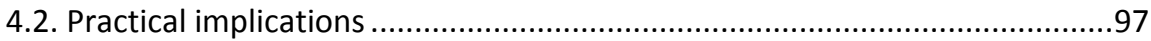

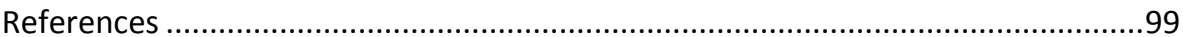

CHAPTER 5 THE PERSONAL DEVELOPMENT PLAN QUESTIONNAIRE (PPQ): THE DEVELOPMENT AND VALIDATION OF AN INSTRUMENT TO ASSESS EMPLOYEES' PERCEPTION OF THE PDP PRACTICE

Based upon Beausaert, S., Segers, M., Gijselaers, W. (2011). The Personal Development Plan Practice Questionnaire (PPQ): The development and validation of an instrument to assess employees' perception of the personal development plan practice. International Journal of Training and Development, 15 (5). Published online.

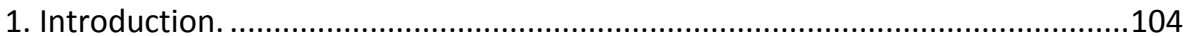

1.1. A Personal Development Plan: Toward a definition ................................105

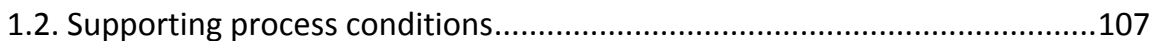

1.2.1. Contextual supporting process conditions ......................................107

1.2.2. Individual supporting process conditions ...........................................110

2. Questionnaire development: Constructs and measures ...............................111

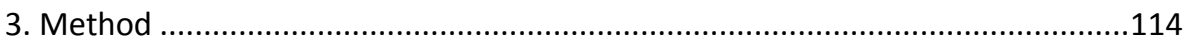

3.1. Sample: Contexts and participants ..........................................................114

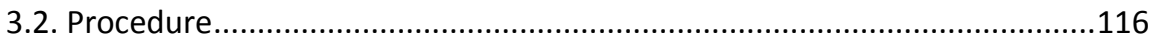

3.3. The instrument: Measures.........................................................................116

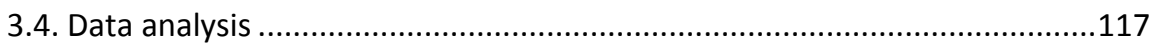

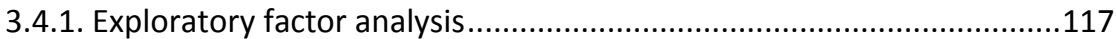

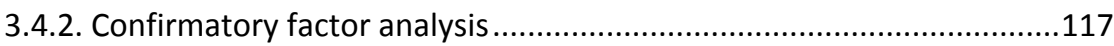

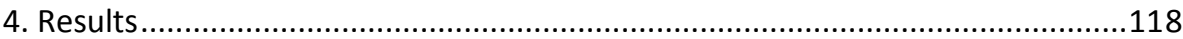

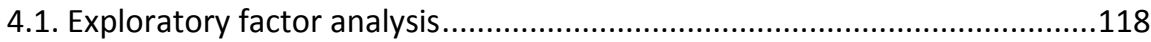

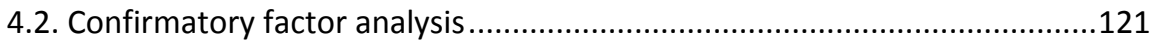

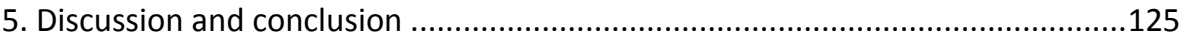

5.1. Limitations and suggestions for future research .....................................126

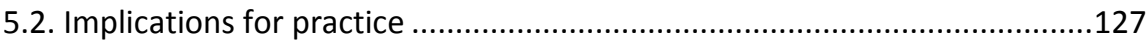

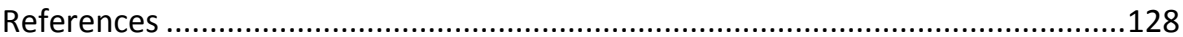

CHAPTER 6 USING A PDP: THE ROLE OF SUPPORTING ASSESSMENT CONDITIONS

Based upon Beausaert, S., Segers, M., \& Gijselaers, W. (accepted with revisions). Using a PDP: The role of supporting assessment conditions.

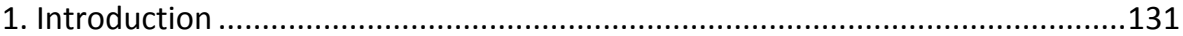

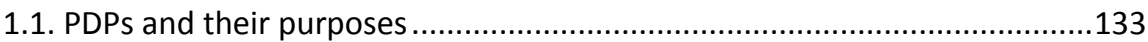

1.2. The PDP practice: Supporting conditions...............................................134

1.2.1. Supporting condition 1: The employee's learning and reflection..........134 
1.2.2. Supporting condition 2: Providing information and feedback. .134

1.2.3. Supporting condition 3: The motivating supervisor. 136

2. Method .137

2.1. The participating organization and its employees..... 137

2.2. Measures 138

2.3. Method of analysis. 140

3. Results. 141

3.1. Preliminary analysis 141

3.2. The influence of the PDP components on the outcome variables 141

3.3. The mediating role of the extent to which employees undertake learning activities 144

4. Conclusion and discussion 146

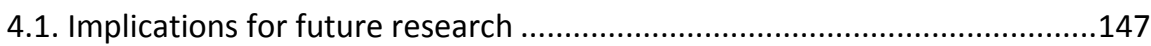

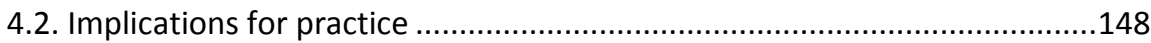

References 150

\section{CHAPTER 7 GENERAL CONCLUSION AND DISCUSSION}

1. Beyond popularity: Looking for empirical evidence 154

2. Results: Empirical evidence on the effects, purposes and supporting conditions of PDPs 155

2.1. Is the PDP effective for the undertaking of learning activities and the employee's performance? 155

2.2. Which goals is a PDP used for and how does the employee's perception of those goals influences the employee's undertaking of learning activities and performance? 157

2.3. Do three supporting conditions - the employee's learning and reflection, the instruction and feedback given by the supervisor, and the way the supervisor motivates the employee - influence the undertaking of learning activities, expertise-growth and performance? 158

3. Practical implications for Human Resource Management and development ....160

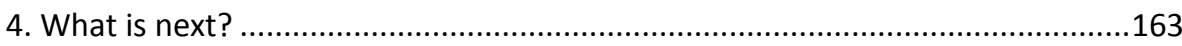

References 167

\section{ABOUT THE AUTHOR}

1. Biography 169

2. List of publications and presentations 169

\section{SAMENVATTING}


1. De populariteit voorbij: Op zoek naar empirisch bewijs.

2. Resultaten: Empirisch bewijs voor effectiviteit, doelen en ondersteunende condities

2.1. Is het gebruiken van een POP effectief voor het ondernemen van leeractiviteiten en de prestatie van de werknemer?

2.2. Voor welke doelen wordt een POP gebruikt en hoe beïnvloedt de perceptie van de werknemer van deze doelen het ondernemen van leeractiviteiten en de prestatie?

2.3. Beïnvloedt de POP-praktijk, gekenmerkt door drie inherente ondersteunende condities (leren en reflecteren van de werknemer, instructie en feedback gegeven door de leidinggevende en de manier waarop de leidinggevende motiveert) het ondernemen van leeractiviteiten, expertisegroei en prestatie? 176

3. Praktische implicaties voor Human Resource Management en development...178

4. Wat nu?. 180

References 184 


\section{List of Figures and Tables}

$\begin{array}{lll}\text { Figure } 1.1 & \text { The relations researched in Chapter } 3 & 20\end{array}$

$\begin{array}{lll}\text { Figure } 1.2 & \text { The relations researched in Chapter } 4 & 21\end{array}$

Figure 1.3 The relations researched in Chapter 622

Table 2.1 Search terms and their combinations 31

Table 2.2 Number of references in different contexts 34

Table 2.3 Integrated table: Goals, effects and supporting process conditions 42

Table 2.4 Number of references according to the research method 51

Table 2.5 Studies sorted according to the number of participants 52

Table 2.6 PDP Assessment in the workplace: Which goals are being strived for? 53

Table 2.7 Which goals is PDP assessment effective for? 54

Table 2.8 Supporting process conditions: Object of study 55

Table $2.9 \quad$ Supporting process conditions mentioned in literature 56

Table 3.1 Descriptive statistics and ANOVAs for users and non-users of a PDP 66

Table 3.2 Summary of logistic regression analysis for PDP use predicting Undertaking learning activities, controlling for background variables

Table 3.3 Summary of hierarchical regression analysis for PDP use predicting Planning future learning activities, controlling for background variables 68

Table 4.1 Overview of the different scales and their descriptives $\quad 87$

Table $4.2 \quad$ Correlations 88

Table 4.3a Hierarchical regression analysis of the independent personal learning and development purposes scale on the dependent variables

Table 4.3b Hierarchical regression analysis of the independent organizational learning and development purposes scale on the dependent variables

Table 4.3c Hierarchical regression analysis of the independent Promo tion and selection purposes scale on the dependent variables

Table 4.4 Hierarchical regression analysis of all purpose components on the dependent variables

Table 4.5 Analysis of variance between Organization 1 and

Organization 2 
Figure 5.1

Table 5.1

Table 5.2

Table 5.3

Figure 5.2

Table 5.4

Table 5.5

Figure 6.1

Table 6.1

Table 6.2

Table 6.3a

Table 6.3b

Table 6.3c

Table 6.4

Table 6.5

Figure 7.1

Table 7.1

Table 7.2
Conceptual model

113

Overview of the variables

114

Exploratory factor analysis PDP practice: Pattern matrix

119

Descriptives for the different scales, resulting from the exploratory factor analysis

121

Confirmatory factor analysis: Factors and their items

123

Confirmatory factor analysis: Factors and their items

Descriptives for the different scales, resulting from the confirmatory factor analysis

Hypothetical model for the effect of the PDP practice components on the outcome variables: undertaking learning activities, expertise-growth, flexibility and performance

Overview of the different scales and their descriptives

Correlations

Hierarchical regression analyses of the learning and

reflection component on the dependent variables:

undertaking learning activities, expertise-growth and performance

Hierarchical regression analyses of the instruction and feedback component on the dependent variables: under taking learning activities, expertise-growth and performance

Hierarchical regression analyses of the motivating supervisor component on the dependent variables:

undertaking learning activities, expertise-growth and performance

Mediation analysis with the PDP component learning and reflection as independent variable

Mediation analysis with the PDP component the motivating supervisor as independent variable

Overview and integration of the three main research questions

Steps to take when implementing a PDP

Comparing the organizational characteristics of the organizations involved in the research project 



\section{CHAPTER 1 INTRODUCTION}

In a non-profit governmental organization 30,000 employees are working in 13 different regions. In this case we will focus on one of those regions where 1400 employees are located in five different offices in four different cities. While it is a very large, bureaucratic organization, it is described by employees and management as a warm family business because most employees who start working there, enjoy the work and are so dedicated to the organization that they keep working for the organization for the rest of their lives. This has led to an average age of the employees of 49 years. This implies that in 2012 (and the following years) the organizations will encounter a large stream out. As a consequence, talent management and continuing professional development of the junior staff are high on the strategic agenda. Furthermore, as an effect of computerization, reorganizations are regularly implemented. This increases the need for taking care of the continuing professional development of employees.

Most employees working in the organization annually undergo an assessment cycle. Formally the assessment cycle consist of a performance interview, a development interview and an appraisal interview with the supervisor. In that assessment process the supervisor and the employee can make use of a Personal Development Plan (PDP). The PDP lines up the competencies the employee still needs to develop (looking forward) through evaluating his or her current strengths and weaknesses (looking back). In the PDP-form the following questions are asked: 'What are your weaknesses?', 'What are your strengths?', 'What do you want to accomplish?', 'Which competencies do you still need to develop?' and 'How do you want to develop those competences?' The answers to these questions can be used to nourish the dialogues with the supervisor, in which the supervisor will try to stimulate the employee's reflection on the PDP. The dialogue with the supervisor and the employee's reflection is crucial and forms the core of using a PDP. Reflection has several purposes. First, the employee should get a better insight in what he or she wants (e.g. making promotion or keep doing the same job and learning 
how to deal with new technologies). Second, keeping in mind the goal of the employee, reflection should lead towards insights in the employee's strengths and weaknesses that will help or hinder him or her in accomplishing the defined goal. Most employees are not aware of their strengths and weaknesses, since they were never encouraged to explicitly think about them, for example during dialogues with colleagues or supervisors. Third, reflection should help the employee to keep track of learning activities undertaken, ranging from following a training to looking something up on the internet, and make sure employees think about what they learned during those learning activities and how. Most employees only think of learning activities as undertaking training. Most of them are not aware of the fact that they learn everyday and how they do this. Furthermore, to be able to transfer what was learned off-the-job, follow-up reflection is crucial. The PDP supports the employee in tracking, structuring and thinking about the undertaken learning activities and consequently, consciously plan future learning activities if necessary. In the end the employee will have worked on his or her professional development and be able to accomplish the set goals.

Nevertheless, while a smaller group of employees is very enthusiastic and motivated to use the tool, most supervisors and employees perceive doing the interviews and using the tool as a burden. They are very skeptical about the effects of the tool and about the reflection the tool should nourish. Following remarks are not uncommon: 'Using a personal development plan leads to nothing. It is frustrating and not motivating. I fill in the form against my will every year again. Why are they torturing us with this tool? It's not that I do not know what to do with my time. Furthermore, my supervisor is not at all occupied with my development.' In other words, many supervisors and employees believe they do not need a tool in order to have good conversations with their employees and supervisors, respectively. It seems as if they think they do not need a PDP in order to talk about their learning and they believe they do not need to be 'forced' to reflect. Consequently, the tool is not strictly used by every supervisor. Most of the experienced supervisors develop their own way of conducting the interviews and using the instrument. As a consequence, a lot of variation exists in how the conversations are conducted and how the tools are used, which of course does not make it any clearer for the employees. Next, many supervisors, especially the younger ones, are not really familiar with the rationale behind the interviews and the forms and are at a loss how to conduct these kind of conversations.

In sum, while the HR department has high expectations of performance interviews and personal development plans and a group of employees enjoy using a PDP, some of the employees and supervisors do not really see the advantages. As a consequence, the tool is not properly implemented and used and brings perceived ineffectiveness, frustration and window dressing. 
This example shows that a tool is only as powerful as it is implemented. Although literature on the subject argues many benefits of the tool, experiences with it, as illustrated in the example as well as a few former research studies, indicate that positive effects can be jeopardized by the way the PDP is implemented. This observation was the starting point for the research reported in this book. In this first chapter we will firstly look into the reasons for using PDPs in the labour market from both a practical and a theoretical point of view. Secondly, we will define what a PDP is, exactly. Finally, we will give an overview of the dissertation and introduce the different research questions that the following chapters will answer.

\section{Continuing professional development and PDPs: Practice and theory}

Organizations in general have been facing and dealing with a variety of challenges. The current labour market is characterized by a growing number of short-term contracts, a shortage of skilled workers in some industries, an ageing workforce and increasing employee mobility. As McDowall and Fletcher formulate it (2004, p. 8): "It is in an organization's best interest to focus on effective staff development strategies, since companies strong on training and development may be preferred (by employees) over those offering the greatest rewards. One technique for developing individuals is through the social and motivational aspects of appraisal or review processes." They define appraisal processes in relation to the formal rating of performance, whereas the term review is used to refer to human resource practices that support employees' professional growth or development. Investment in human capital does not only serve the purpose of the attraction and retention of high quality employees, however, it is also described as a crucial strategic tool for organizations to be competitive in an environment that is characterized by ongoing innovation. Continuous innovation demands for continuous development of employees' expertise and flexibility towards changing circumstances since employees nowadays rarely fulfill the same job or function in the same organization for the rest of their lives.

The increasing emphasis on the strategic role of learning and development has challenged Human Resource departments to develop and implement tools that support employees in the undertaking of learning activities that contribute not only to an improved performance but also to the employee's expertise growth and flexibility. Popular techniques are multi-source or 360-degree feedback, career discussions with managers and Personal Development Plans (PDPs). However, as McDowall and Flatcher (2004) indicate, most studies to date have tended to concentrate on performance or rewards rather than development, perhaps reflecting the tradi- 
tional practice of retrospective assessment (Nathan, Mohrmann, \& Milliman, 1991).

In this PhD thesis, we focus on Personal Development Plans, a strategic developmental tool used by Human Resource departments to stimulate employees' formal (e.g. training) and informal (e.g. reading a book, peer feedback discussions) learning, which in turn is assumed to improve expertise-growth and performance. In general a PDP can be described as an assessment tool embedded in a larger assessment cycle of development and appraisal interviews, used to gather and document information about the competencies the employee worked on and is planning to further develop (London, 1997; van de Wiel, Szegedi, \& Weggeman, 2004).

PDPs have come into widespread use, not only in the fields of medicine and education, but also in business contexts and government offices (Beausaert, Segers, van der Rijt, \& Gijselaers, 2011). For example, a survey in the Netherlands among companies in the region of Limburg indicated that $89 \%$ of the companies are using PDPs in the context of talent management (GITP, 2008). In the UK, different authors stress the strong recommendation by the government to use PDPs in order to stimulate the continuous professional development of health service (Evans, Ali, Singleton, Nolan, \& Bahrami, 2002; Bullock, Firmstone, Frame, \& Bedward, 2007).

Despite the popularity of PDPs in the workplace, relatively little is known about the actual use of the tool and its impact on the employee's learning and development. Empirical evidence on the impact or effectiveness of PDPs is limited (e.g. Austin, Marini, \& Desroches, 2005; Evans, et al., 2002; Zeichner \& Wray, 2001). As a consequence, the implementation of the tool by Human Resource Management (HRM) is accompanied by different assumptions and hypotheses. While the idea behind PDPs is that they should stimulate the employees' learning and professional development, practice shows that in many cases the opposite seems to be true. Employees perceive the tool as ineffective, part of an annual ritual dance or an alibi serving as cover-up to mask that when it comes to personal development organizations don't really care much (Leggett \& Bunker, 2006; Stone, 1998; Challis, 2001).

In sum, PDP assessment in the workplace has become popular, yet, little is known about the practical use of the tool. In order to contribute to the optimization of PDP practices and enhance their effectiveness, it is clear that more research is needed.

\section{A Personal Development Plan (PDP): Definition}

The concept Personal Development Plan (PDP) is related to various concepts such as portfolio, (continuing professional or personal) development plan, logbook or 
personal professional profile. The two most commonly used terms are personal or professional development plan and portfolio assessment. With respect to the latter, different connotations can be discerned. In many literature sources, portfolio (assessment) refers to a report system that is used for organizational accountability and learning (Schmitz \& Schillo, 2005). Furthermore, a portfolio is described as a record that provides evidence of acquisition of skills, knowledge, attitudes, understanding and achievements (Brown, 1995; Redman, 1994) or competencies of employees (Smith \& Tillema, 1998). On the one hand it demonstrates development or 'continuing' acquisition; it is prospective (Brown, 1995, McMullan et al., 2003). On the other hand a portfolio is retrospective, considering reflection takes a central place in constructing one (Brown, 1995; Snadden, Thomas, Griffin, \& Hudson, 1996).

Portfolios originally were used as a showcase by photographers, painters, architects and brokers (Lyons \& Evans, 1997; Mathers, Challis, Howe, \& Field, 1999). Later, they were implemented in secondary schools and higher education to support student learning and to inform certification. One section of the portfolio was dedicated to the professional development of the students, referred to as the PDP (e.g. Driessen, van Tartwijk, van der Vleuten, \& Wass, 2007). Gradually teachers, nurses and general practitioners also started using portfolios. In contrast to its use in school settings, however, making improved professional rather than educational performance visible in a portfolio is not easy. This is also reflected in the discussion on how to operationalize outcomes of training, a discussion which has been going on for years. Instead, portfolios in professional settings are used as a tool used to present information about the competencies the employee has been working on and is planning to further develop and for this reason labeled as a Personal Development Plan. The PDP can be defined as a tool that:

- Gives an overview of the competencies the employee worked on in the past and which competencies the employee is planning to work on in the future;

- Is composed by the employee himself (self-direction by the employee) although the format of the PDP is mostly fixed;

- Is used as basis for or to structure the conversations with the supervisor or the coach, who provides the employee with feedback and stimulates the employee's reflection;

- $\quad$ Serves for taking different decisions, going from planning an individual training program to whether or not giving someone a promotion.

In this book we will research the Personal Development Plan as defined by these four characteristics. The main aim of the project is to research under which circumstances a PDP is most effective for continuing professional development. 


\section{Overview of the dissertation and research questions}

Before turning to the research questions a literature review is conducted in order to define a PDP and to get an overview of the available empirical research on the goals, the effects and the personal (e.g. the employee's competency to reflect) and environmental (e.g. the supervisor who is supporting the employee in using the tool) supporting conditions of PDPs (Chapter 2). Second, the first and main research question is researched: 'Is the PDP effective for the undertaking of learning activities and the improvement of the employee's performance?' (Chapter 3). In this study pharmacy assistants who are using a PDP were compared with pharmacy assistants who are not (Figure 1).

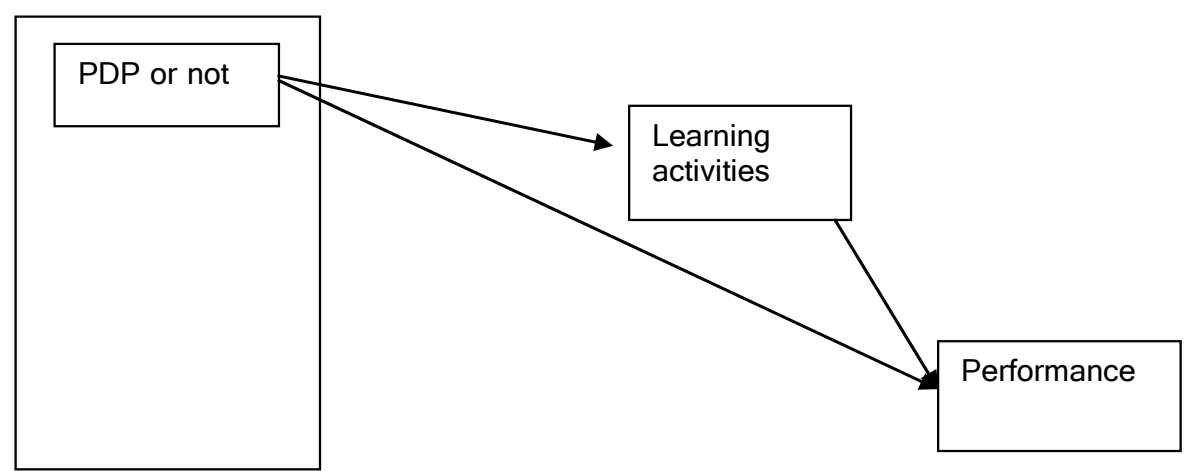

Figure 1

The relations researched in Chapter 3

However, the literature review in Chapter 2 shows that PDPs are used for different goals and that personal and environmental supporting conditions may influence the effectiveness of the tool. This led to our second and third research questions: 'Which goals is a PDP used for and how does the employee's perception of those goals influence the undertaking of learning activities and performance?' (Chapter 4) and 'Does the PDP practice, characterized by three supporting conditions (the employee's learning and reflection, the instruction ${ }^{1}$ and feedback given by the supervisor, and the way the supervisor motivates the employee), influence the undertaking of learning activities, expertise-growth and performance?' (Chapter 6).

The study described in Chapter 4, in which the influence of the perceived goals was researched and which was conducted in both a profit and a non-profit organization, indicated that perceiving the tool both as a learning and development tool and as a selection and certification tool influences the undertaking of learning activities and performance positively. However, perceiving the PDP as a learning and development tool had a stronger impact (Figure 2).

1 In non-educational journals 'Instruction' was replaced by 'Information'. 


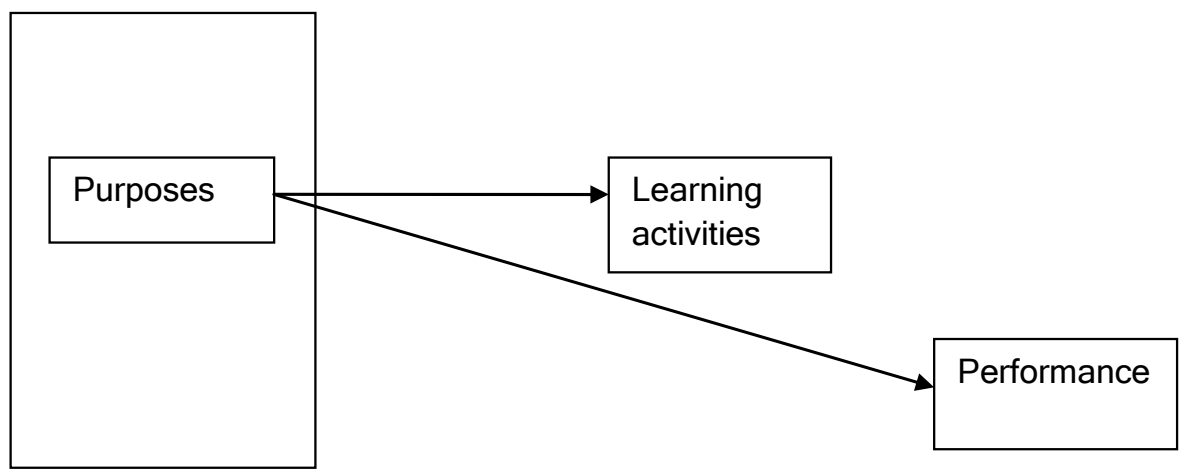

Figure 2

The relations researched in Chapter 4

In order to formulate an answer to the next research question 'Does the PDP practice influence the undertaking of learning activities, expertise-growth and performance?' (Chapter 6) an instrument that measures the PDP practice had to be developed and validated first (Chapter 5 ). The validation study conducted in two profit organizations and one non-profit organization resulted in the PDP Practice Questionnaire (PPQ), consisting of three relevant scales, measuring three supporting conditions of the PDP practice: the employee's learning and reflection (personal condition), the supervisor's instruction and feedback (environmental condition) and the way the supervisors motivate the employee (environmental condition). The newly developed questionnaire was used in the study described in Chapter 6 in order to measure the PDP practice in a non-profit organization. Next, the effects on the undertaking of learning activities, expertise-growth and flexibility and performance were measured. In general it was found that a motivating supervisor, his or her instructions and feedback and reflection by the employee on the basis of his/her PDP influence whether a PDP leads to the measured output variables. Next, evidence was found for the mediating role of undertaking learning activities in the relation between the PDP practice (learning and reflection and motivating supervisor) and two output variables (expertise-growth and performance) (Figure 3). Finally, Chapter 7 summarizes the concepts, results, implications for future research and the practical implications of this research project. 


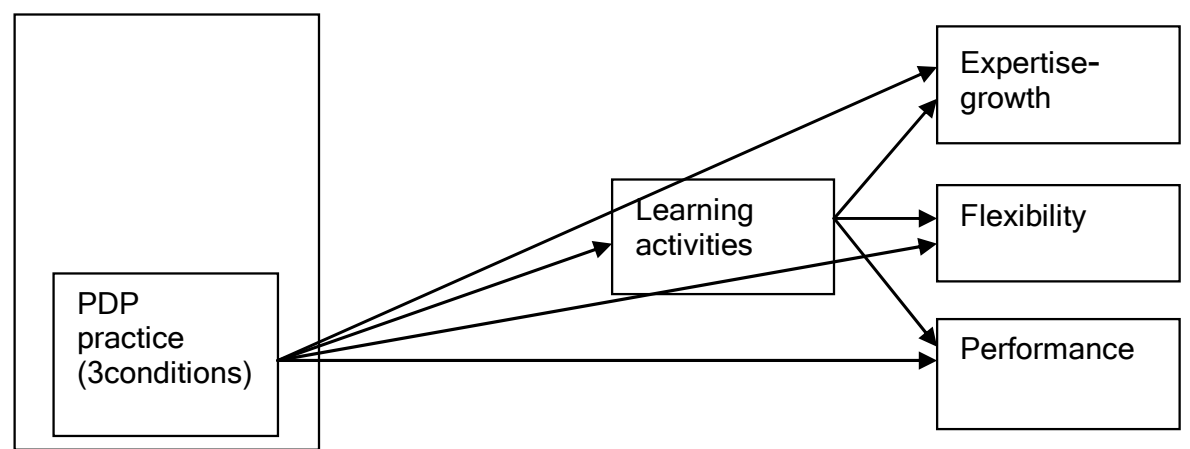

Figure 3

The relations researched in Chapter 6

The following chapters are based on journal articles and a book chapter and discuss each research question separately. Considering every article is written to be read on its own, repetitions and overlap across chapters are inevitable. 


\section{References}

Austin, Z., Marini, A., \& Desroches, B. (2005). Use of a learning portfolio for continuous professional development: A study of pharmacists in Ontario (Canada). Pharmacy Education, 5, 175-181.

Beausaert, S., Segers, M., van der Rijt, J., Gijselaers, W. (2011). The use of Personal Development Plans in the workplace: A literature review. In P. van den Bossche, W. Gijselaers, \& R. Milter (Eds.), Building learning experiences in a changing world, Advances in Business Education and Training III (pp. 235-265). Dordrecht: Springer.

Brown, R. (1995). Portfolio development and profiling for nurses, $2^{\text {nd }}$ edn. Lancaster: Quay Publications.

Bullock, A., Firmstone, V., Frame, J., \& Bedward, J. (2007). Enhancing the benefit of continuing professional development: A randomized controlled study of personal development plans for dentists. Learning in Health and Social Care, 6 (1), 14-26.

Challis, M. (2001). Portfolios and assessment: Meeting the challenge. Medical Teacher, 23, 437-440.

Driessen, E.W., van Tartwijk, J., van der Vleuten, C.P.M., \& Wass, V.J. (2007). Portfolios in medical education: Why do they meet with mixed success? A systematic review. Medical Education, 41, 12241233.

Evans, A., Ali, S., Singleton, C., Nolan, P., \& Bahrami, J. (2002). The effectiveness of personal education plans in CPD: An evaluation. Medical Teacher, 24 (1), 79-84.

GITP (2008). HR(D) in Limburgs perspectief. [HRD in Limburg, the Netherlands]

Leggett, M., \& Bunker, A. (2006). Teaching portfolios and university culture. Journal of Further and Higher Education, 30, 269-282.

London, M. (1997). Job feedback: Giving, seeking, and using feedback for performance improvement. Mahwah, NJ: Erlbaum.

Lyons, N., \& Evans, L. (1997). Portfolio: A tool for self-directed learning at work. Paper presented at the Self-directed Learning: Past and Future Symposium (Montreal, Quebec, Canada, September 1997).

Mathers, N.J., Challis, M.C., Howe, A.C., \& Field, N.J. (1999). Portfolios in continuing medical education effective and efficient? Medical Education, 33, 521-530.

McDowall, A., \& Fletcher, C. (2004). Employee development: An organizational justice perspective. Personnel Review, 33: 8-29.

McMullan, M., Endacott, R., Gray, M.A., Jasper, M., Miller, C.M.L., Scholes, J., \& Webb, C. (2003). Portfolios and assessment of competence: A review of the literature. Journal of Advanced Nursing, 41, 283-294.

Nathan, B.R., Mohrmann, A.M., \& Milliman, J. (1991). Interpersonal relations as a context for the effects of appraisal interviews on performance and satisfaction: A longitudinal study. Academy of Management Journal, 34, 352-369.

Redman, W. (1994). Portfolios for development: A guide for trainers and managers. London: Kogan Page. Schmitz, C.C., \& Schillo, B.A. (2005). Report Carding: A model for foundation portfolio assessment. Americain Journal of Evaluation, 26, 518-531.

Smith, K., \& Tillema, H. (1998). Evaluating portfolio use as a learning tool for professionals. Scandinavian Journal of Educational Research, 42, 193-205.

Snadden, D., Thomas M.L., Griffin, E.M., \& Hudson, H. (1996). Portfolio-based learning and general practice vocational training. Medical Education, 30, 148-152.

Stone, B.A. (1998). Problems, pitfalls, and benefits of portfolios. Teacher Education Quarterly, 25 (1), 105-114.

Van de Wiel, M.W.J., Szegedi, K.H.P., \& Weggeman, M.C.D.P. (2004). Professional learning: Deliberate attempts at developing expertise. In: H.P.A. Boshuizen, R. Bromme, H. Gruber (Eds.) Professional Learning: Gaps and transitions on the way from novice to expert (pp. 181-206). Dordrecht, the $\mathrm{Ne}-$ therlands: Kluwer.

Zeichner, K., \& Wray, S. (2001). The teaching portfolio in US teacher education programs: what we know and what we need to know. Teaching and teacher education, 17, 613-621. 



\section{CHAPTER 2}

\section{THE USE OF PERSONAL DEVELOPMENT PLANS IN THE WORKPLACE: A LITERATURE REVIEW ${ }^{2}$}

It is evident that in the current knowledge economy learning does not stop after graduation. Learning for a profession is only a starting point for learning in the profession. One tool to enhance learning in the profession is a personal development plan (PDP). Although this tool is very popular, to date, there is no review study available to inform researchers and practitioners about effects and conditions enhancing the effectiveness. Therefore, we conducted a systematic narrative literature review, selecting empirical studies to research PDPs in the workplace. The purpose of this review-study was to analyze the literature on the goals that PDP assessment is being used for in the workplace, its effectiveness to reach those goals and the desirable supporting conditions that will enlarge its effectiveness. The results highlight nine clusters of goals PDP assessment is used for, namely: professional development; reflective learning; providing evidence; documenting; certification, selection and promotion; external mobility; coaching; stimulating confidence; and organizing. Furthermore, the small amount of empirical evidence on the effectiveness of PDP assessment ( $N$ studies $=54$ ) indicates that the instrument is effective for personal or continuing professional development, stimulating reflection and improving professional practice. There nevertheless exists a gap between the popularity of this tool and the availability of evidence of its effectiveness. Moreover, almost all studies researching PDPs took place in an educational or healthcare setting. Finally, only the support received from a coach or supervisor and the time and resources provided have been researched as supporting process conditions and were found to have positive effects. In most other included articles the supportive conditions are no object of study but are theoretically discussed and argued upon. It is clear that to inform Human Resource Development professionals using PDPs, more studies systematically investigating the effects of the tool and the supporting process conditions are necessary.

2 Based upon Beausaert, S., Segers, M., van der Rijt, J., Gijselaers, W. (2011). The use of Personal Development Plans in the workplace: A literature review. In P. van den Bossche, W. Gijselaers, \& R. Milter (Eds.), Building learning experiences in a changing world, Advances in Business Education and Training III (pp. 235-265). Dordrecht: Springer. 


\section{Introduction: A PDP as a trojan horse?}

The dynamic nature and the aging population of today's knowledge economy has forced organizations to put the issues of talent management and employability high on the strategic agenda. After all, the talents of employees, and more specifically the competencies they possess, drive the success of the current business (Guthridge, Komm, \& Lawson, 2008). Moreover, employable professionals or employees who possess "the qualities and competencies required to meet the changing needs of employers and customers, and thereby help to realize his or her aspirations and potential in work" (CBI, 1999, p. 1), are the organizations' greatest asset. To that end, organizations implement various tools (e.g. annual appraisal interviews, in-company training, 360-feedback) to support the development of these talents and competencies and to extract the most from their employees. In this respect, organizations to a growing extent make use of a Personal Development Plan (PDP) to foster workplace learning.

This literature review aims at providing an overview of the goals PDPs are used for, their effects and the supporting conditions which are desirable in order to optimize the use of the tool. In the following paragraphs we will first define a PDP. Second, we will discuss the different goals the tool is used for. Third, we will link the PDP with the principles of adult learning. Fourth, we will elaborate on the necessity of support when using a PDP. Finally, the goal and research questions of this literature review will be discussed.

\subsection{A Personal Development Plan: Toward a definition}

In literature the concept Personal Development Plan (PDP) is referred to by different synonyms: portfolio, (continuing professional or personal) development plan, logbook or personal professional profile. The two most commonly used terms are personal or professional development plan and portfolio assessment. With respect to the latter, different connotations can be discerned. In many literature sources, portfolio (assessment) refers to a report system that is used for organizational accountability and learning (Schmitz \& Schillo, 2005). Therefore, in order to avoid misunderstandings about the concept, we will use the term Personal Development Plan or PDP.

The PDP idea is complex and has been interpreted in many ways (Wildy \& Wallace, 1998). The same terms are often used to indicate instruments with different characteristics and ranges of application. In spite of the differences, those instruments have several characteristics in common ${ }^{3}$. Based on the literature a PDP can

3 For clarity reasons we will use the term 'personal development plan' or 'PDP' in this article, even if the literature source we are referring to uses one of the synonyms we used as search terms in the systematic literature search process (see methodology section). 
be defined as an assessment tool, used by employees in organizations, that (Brown, 1995; McMullan et al., 2003; Redman, 1994; Seng \& Seng, 1996; Smith \& Tillema, 1998; Snadden, Thomas, Griffin, \& Hudson, 1996):

- gives an overview of the competencies the employee worked on in the past and which competencies the employee is planning to work on in the future and how;

- should be composed by the employee himself, mostly in consultation with the supervisor;

- can be used as a basis/structure for conversations with the supervisor or coach, who provides the employee with feedback and stimulates the employee's reflection; and

- $\quad$ serves as a decision-making tool, from planning an individual training-program to assessing the suitability of a promotion.

The above mentioned characteristics of a PDP indicate that when an employee sums up the competencies he ${ }^{4}$ has already developed, he becomes aware of the competencies he possesses and which direction he wants to take in his professional career. Furthermore the employee may see this as an opportunity to develop other competencies he is still lacking to reach a certain goal (e.g. promotion). In that case using a PDP should lead towards undertaking learning activities. As a consequence it can be expected that the employee's expertise will grow and his performance will improve. The supervisor plays an important role in stimulating the employee's reflection (Boud, Keogh, \& Walker, 1985) (see 1.4. Using a PDP needs support). In the box below we present an example of an organization in which the employees are using a PDP as a tool within an assessment cycle.

Consider a non-profit governmental organization in the Netherlands, employing 30.000 workers in 13 different regions. In one of those regions, 1400 employees are located in 5 different offices in 4 different cities. While the organization is a very large and bureaucratic organization, it is described by employees and management as a warm family business because the majority of employees who start working there, enjoy the work and are so dedicated to the organization that they keep working for it for the rest of their lives. Consequently, the average age of the employees is 46.6 years. In 2012 the organization will therefore be faced with a very large stream out. It is for this reason that talent management and continuing professional development of the staff is high on the strategic agenda.

Most employees working in the organization undergo an assessment cycle on an annual basis. The assessment cycle consists of a performance interview, a de-

$4 \quad$ For clarity reasons we will use the term 'he' when we refer to the employee, while it should be 'she or he'. 
velopment interview and an assessment interview with the supervisor. In this assessment cycle the supervisor guides the employee in using a Personal Development Plan (PDP). The PDP format asks for the competences the employee needs to develop (looking forward) through evaluating the strengths and weaknesses of the employee (looking back). In the form the following questions are posed: 'What are your weaknesses?', 'What are your strengths?', 'What do you want to accomplish?', 'Which competencies do you still need to develop?', 'How do you want to develop those competencies and in which timeframe?' and 'Which support is needed?'. The answers to these questions are used to nourish the interviews with the supervisor, in which the supervisor will try to stimulate the employee's reflection on his or her PDP.

\subsection{What is the purpose of PDP assessment?}

A PDP is a tool used to gather and document information about the competencies the employee worked on and is planning to further develop. In this respect, it can be defined as an assessment tool. Gathering and documenting information by means of a PDP can serve two main purposes. Commonly a distinction has been made between two main purposes: professional development and certification/selection/accountability (Smith \& Tillema, 2001). When a PDP is used to develop, learning takes a central role. Conversely, when a PDP is used for certification, presenting oneself is most important (Beck, Livne, \& Bear, 2005; Bradshaw \& Hawk, 1996; Bunker \& Leggett, 2004; Lyons \& Evans, 1997; Orland-Barak, 2005; Smith \& Tillema, 2001). Smith and Tillema (2001) refer to two dimensions. The first continuum goes from the "certification (promotion, selection) purposes" to the "learning, developmental purposes". The second continuum makes a difference between composing a PDP on a voluntary basis and on a mandatory basis. The authors reported that there was more professional development when sustained PDP use was voluntary than when it was mandatory (Smith \& Tillema, 2001). In contrast, other research showed that staff members did not develop a [teaching] PDP unless they were obliged to (mandatory) (Bunker \& Leggett, 2004). In line with the distinction made between the two main purposes, a difference has been made between several types of portfolios. A working portfolio is used for reflection, while a documentary portfolio and a show portfolio are used for summative assessment. The difference between a documentary portfolio and a show portfolio lies in the fact that a show portfolio only displays the best of someone's work (Bunker \& Leggett, 2004). Another similar difference has been made between a product or showcase portfolio and a process portfolio (Moore \& Bond, 2002). 


\subsection{PDPs and principles of adult learning}

A PDP fits well the assumptions of the theory of adult learning (Knowles, 1975). First, the assessment tool assumes that an employee is curious and self-motivated to develop (Joyce, 2005). Therefore, it stimulates the employee to take responsibility for his or her own learning (Lyons \& Evans, 1997). In other words, a PDP supports a self-directed way of learning; the employees should regulate their learning processes themselves. Self-directed learning is: "a process in which individuals take the initiative in evaluating their learning outcomes, diagnosing learning needs, formulating learning goals, and selecting appropriate learning tasks" (Kicken, Brand-Gruwel, van Merriënboer, \& Slot, 2008). Second, it allows the employee to use his previous experiences as rich resources for his or her learning. Based on experience the employee has already gathered a lot of (tacit) knowledge of which he is often unaware. By reflection on those experiences the employee's knowledge can be brought to the surface and developed. Alsop (2002) describes reflections as: "the images of our experiences, revisited for the purpose of learning" (p.203). Kolb (1984) too stated that learning occurs through "concrete experiences" (e.g. performing a job) and reflecting on what has been experienced, which can be stimulated during a meeting with the supervisor. Third, the PDP allows the employee to develop readiness to learn from life tasks and problems. By undertaking life tasks and problems the employee is provided with stepping stones to stimulate his learning.

\subsection{Using a PDP needs support}

Using a PDP needs to be supported. An employee needs to be supported in a noncontrolling way that empowers self-development and should be provided with behavioral choices for learning (London \& Smither, 1999). In turn, this leads to an increased intrinsic motivation, as the employee experiences a sense of selfcompetence and feels in control of his own behavior. Feedback seeking and participation in learning activities should be encouraged and rewarded as well (London, Larsen, \& Thisted, 1999; London \& Smither, 1999). Support can be offered by a coach, a mentor or a supervisor. In organizations the assessment process in which a PDP is used is mostly supported by the supervisor. This is in line with research that found that in using a dossier, training and reflective PDP external feedback was most valued when it was given by a superior (Smith \& Tillema, 2003). Furthermore, it was found that employees are more likely to engage in developmental activities such as trainings when they have supervisors that are supportive of their efforts (London et al., 1999).

A supervisor should be available in order to have sufficient contact with the employee and provide feedback (Wasylyshyn, 2003). The lack of interaction with the supervisor is mostly due to time limitations, incompatible work schedules, and 
physical distance (Noe, 1988). Additionally, it is found that when the supervisor provides his or her employees with a clear goal, a formal framework (including meetings), specific guidelines and standards; the effects of personal development plan assessment will be larger (e.g. Guaglianone, 1995; Noe, 1996; Roberts, 2003). Support can also be given by colleagues (peers). Peers often have a better view on the job performance and development of their colleagues than their supervisors (Beck et al., 2005). Furthermore, employees value exchanging ideas, hearing different viewpoints and exchanging feedback with their colleagues (Tigelaar et al., 2006).

Finally, a supportive environment contributes to the success or failure of the use of a PDP. The more supportive the environment, the more employees are open to receiving guidance in their professional development (Johnston \& Thomas, 2005; Joo, 2005; London et al., 1999). A supportive environment includes the provision of time and resources. Time is needed to compose a PDP and discuss it with the supervisor during assessment interviews. Resources provided could be print- and web-based resources that help construct a PDP, and provision of training in using the tool (Austin, Marini, \& Desroches, 2005; Bradshaw \& Hawk, 1996; Daniel \& Stallion, 1995; Tillema, 1998).

\subsection{The goal of this literature review}

The PDP stems originally from portfolios that were used as a showcase by photographers, painters, architects and brokers for a long time (Lyons \& Evans, 1997; Mathers, Challis, Howe, \& Field, 1999). Later, students in secondary schools and higher education began using portfolios to support their learning and facilitate certification. One section was dedicated to the professional development of the students, referred to as the PDP (e.g. Driessen, van Tartwijk, van der Vleuten, \& Wass, 2007). Recently, PDPs have become increasingly used in organizations as well. Because the PDP holds the promise to be a useful tool in managing the employee's continuing professional development, it can count on a lot of interest (e.g. Firssova, 2006). For example, there exists a vast amount of theoretical and practice-based literature on this subject (e.g. Wright, Knight, \& Pomerleau, 1999) and conferences and international workshops or seminars on portfolio assessment are popular (for example, The 5th International ePortfolio Conference 2007, 27-29 September 2007).

Despite the popularity of PDPs in the workplace, relatively little is known about the actual use of the tool and its impact on the employee's learning and development. Empirical evidence on the impact or effectiveness of PDPs is limited (e.g. Austin, Marini, \& Desroches, 2005; Evans, Ali, Singleton, Nolan, \& Bahrami, 2002; Zeichner \& Wray, 2001). As a consequence, the implementation of the tool by Human Resource Management (HRM) is accompanied by different assumptions and 
hypotheses. While the idea behind PDPs is that it should stimulate the employees' learning and professional development, practice shows that the opposite seems to be true in many cases. Employees perceive the tool as ineffective, part of an annual ritual dance or an alibi serving as cover-up to mask that when it comes to personal development organizations don't really care much (Leggett \& Bunker, 2006; Stone, 1998; Challis, 2001). By implementing PDPs, a Trojan horse enters the organization. The instrument is brought into the organization with great expectations, but once the instrument is implemented the belly of the Trojan horse opens and what we find is frustration, resistance, window dressing, perceived ineffectiveness and irrelevance and a perceived waste of time.

In sum, PDP assessment in the workplace has won popularity. Yet, little is known about the common use of the tool. In order to contribute to the optimization of PDP practices and enhance their effectiveness, it is clear that more research is needed. A comprehensive overview of the state-of-the-art research on this issue is a fruitful starting point for developing new lines of research. In this narrative literature review we first question the goals PDPs are used for. Second, we present an overview of the empirical evidence on the effectiveness of the tool to reach these goals. Third, we describe the supporting process conditions that encourage the effectiveness of PDPs.

\section{Method}

Our literature search started in 2009. Because of their relevance to the subject under review, the following databases were selected: EBSCO (CINAHL, ERIC and PsycINFO), Emerald Insight, InformaWorld, OVID (Medline)/Pubmed, Oxford Journals, ScienceDirect (Elsevier), SpringerLink, SwetsWise, Wiley InterScience, and Web of Science. The search terms used are registered in Table 1.

Table 1

Search terms and their combinations

\begin{tabular}{lr}
\hline Search terms & Hits \\
\hline Portfolio (title) & 3000 \\
Portfolio assessment (title) & 749 \\
Portfolio (title) and organization & 580 \\
Portfolio (title) and workplace & 50 \\
\hline Professional development plan (title) & 89 \\
PDP (title) & 53 \\
\hline
\end{tabular}


To include only articles that have PDP assessment as the main object of study, we searched for the keywords in the 'Title' only. When we searched for the term portfolio (title), a great amount of the articles that the search query delivered concerned portfolio management (organizational level). To exclude those articles we added the term assessment. The search for portfolio assessment resulted in $\mathbf{7 4 9}$ hits. In addition, most articles were situated within an educational context (students). To exclude those articles, we added the terms organization or workplace. Those searches resulted in 580 and 50 hits. Furthermore we looked for the two most used synonyms of portfolio assessment, namely PDP and professional development plan, which resulted in 89 and 53 hits. The other synonyms found in literature - 'logbook', 'personal activity plan', 'profile' and 'personal diary' - delivered no relevant hits (e.g. Bullock et al., 2007). All articles we included in the literature review discussed tools that had the four characteristics we used to define a PDP in common (see p. 3; 1.1. A Personal Development Plan: Toward a definition). In order to find more articles, we also looked at the reference lists of the articles found. Finally we looked for other articles written by the same authors, concerning the same topic and went through our personal library.

The journal articles and presented papers found were retrieved for more detailed evaluation on the basis of the abstracts and put through the test of the inclusion and exclusion criteria until the final studies were identified. The inclusion criteria were: the assessment tool as the object of study, the PDP as studied resembles the four characteristics we used to define the tool (see above), workplace/ organization, postgraduate students and empirical research. Exclusion criteria were: education (school), (undergraduate or graduate) students, descriptive articles, articles published before 1995 (only three empirical articles), articles discussing the validity and reliability of the instrument ${ }^{5}$, articles about portfolio management and book chapters/book reviews/abstracts/ supplements. Following application of the inclusion and exclusion criteria, 57 references were selected for review. Three of them we were unable to retrieve, either via web libraries or personal contact.

First, by making use of a format, the selected references ( $N$ studies $=54$ ) were categorized according to the distinction that was made by Tillema and Smith (2001) between the certification (promotion, selection) purpose ( $\mathrm{N}$ studies $=9$ ) and the learning, developmental purpose $(\mathrm{N}$ studies $=31$ ). Fourteen references were related to both categories. Second, a closer analysis of both categories was completed and resulted in nine clusters. The clusters were evaluated for interrater reliability. A second rater randomly selected ten references, defined the operationalized goal and divided them into the nine categories, making use of the same

Twenty-seven references focused on the validity, reliability and/or assessment criteria of portfolio assessment; which is not the scope of this literature review (e.g. van der Schaaf, Stokking, \& Verloop, 2005). 
format. Focusing on the ultimate goal of the tool discussed, the rater came up with the same categorization.

Finally, we want to remark that the method sections of the qualitative as well as the quantitative researches were often poorly described and limited to frequencies and means. Only three references described effect sizes concerning the effects of PDP assessment (Bullock et al., 2007; Hrisos, Illing, \& Burford, 2008; Tillema, 2001), two references reported effect sizes when comparing different types of portfolios (Beck et al., 2005; Smith \& Tillema, 2003) and finally three references discussed effect sizes concerning different perceptions of the tool between different groups (Little \& Hayes, 2003; Smith \& Tillema, 2001; Sullivan, 2004). Therefore we have chosen to execute a narrative review ${ }^{6}$ and not a statistical meta-analysis.

\section{Results}

\subsection{Sample}

Concerning the sample of literature, almost all articles used the word 'portfolio' ( $\mathrm{N}$ studies $=46$ ), while only a minority of articles used the terms 'personal/professional/practice development plan' ( $\mathrm{N}$ studies $=5$ ), 'personal education plan' ( $\mathrm{N}$ studies $=2$ ) or 'professional growth plan' ( $\mathrm{N}$ studies $=1$ ).

In addition, nearly all studies were conducted in an educational setting (teachers) $(\mathrm{N}$ studies $=32$ ) or a health setting $(\mathrm{N}$ studies $=21)$. Three references were situated within a management context (see Smith \& Tillema, 1998; Tillema, 2001) (Table 2). It is assumed that the concentration of articles on the subject within the educational field and health context is because the instrument has been used in those contexts for much longer (Mathers et al., 1999). It is only in the last decennium that PDPs have been increasingly used in profit and non-profit organizations for purposes other than certification or selection as well, which may explain the lack of research in those settings (Smith \& Tillema, 2001).

6 A literature review is defined as: "The selection of available documents (both published and unpublished) on the topic, which contain information, ideas, data and evidence written from a particular standpoint to fulfil certain aims or express certain views on the nature of the topic and how it is to be investigated, and the effective evaluation of these documents in relation to the research being proposed" (Hart, 1998). 
Table 2

Number of references in different contexts

\begin{tabular}{lr}
\hline Context $^{T^{\prime}}$ & Number of references \\
\hline Education & $\mathbf{3 2}$ \\
Teachers & 22 \\
Administrators (in & 10 \\
schools)/educational lead- & \\
ers/principals & \\
Health & $\mathbf{2 1}$ \\
Pharmacists & 2 \\
General Practitioners/surgeons & 11 \\
Physicians & 1 \\
Dentists & 1 \\
Nurses & 5 \\
Dietetics & 1 \\
Management & $\mathbf{3}$ \\
\hline Total & $\mathbf{5 6}$ \\
\hline
\end{tabular}

The sample consists mostly of qualitative research ( $\mathrm{N}$ studies $=41$ ) or a combination of qualitative and quantitative research methods ( $\mathrm{N}$ studies $=23)^{8}$. More specifically; interviews ( $\mathrm{N}$ studies $=25$ ), portfolio analysis ( $\mathrm{N}$ studies $=16$ ) and focus groups ( $\mathrm{N}$ studies $=5$ ) were the most used qualitative research methods. Questionnaires ( $N$ studies $=21$ ) and surveys ( $N$ studies $=13$ ) were the most used quantitative methods. For a detailed overview, we refer to Table 3 in the Appendices.

Furthermore, the studies were often conducted with a low number of participants. Thirty-seven studies had less than 50 participants, 11 studies had between 50 and 100 participants and only 14 studies had more than 100 participants. See Table 4 in the Appendices for a detailed overview.

\subsection{Which goals are strived for?}

In order to classify the references we made use of a format based on the work of Smith and Tillema (2001). We made a distinction between two purposes: certification/selection/accountability and development. Nine references were connected to the first group of goals, while 31 references were classified under the developmental goal. Fourteen references were connected to both categories. That last observation shows that in a relatively large amount of studies no clear distinction is made between certification and development. In other words, formative assessment and

\footnotetext{
Note that two articles are situated in two different contexts.

Those studies are integrated more than once in the table.
} 
summative assessment are often integrated, while it is mentioned that the PDP will have no effect if this distinction is not made (Bunker \& Leggett, 2004).

Next, the analysis of the selected studies resulted in the following nine clusters of goals (see Table 5 in the Appendices for a detailed overview):

(1) professional development;

(2) reflective learning;

(3) providing evidence;

(4) documenting;

(5) certification, selection and promotion;

(6) external mobility;

(7) coaching;

(8) stimulating confidence; and

(9) organizing.

The most researched goal, professional development ( $\mathrm{N}$ studies $=42$ ), also refers to the broader context in which the PDP is used in the workplace. Professional development can be defined as: "A process of lifelong learning for all individuals [and teams] which enables professionals to expand and fulfil their potential" (Evans et al., 2002). Professional development incorporates personal development. "Personal development cannot be separated from professional development; each rests upon the other. Show me how well you share of yourself, understand your own personal processes and are able to communicate this to others, and I'll know how good or bad your nursing care is" (Barber, 1992, p. 309; in McMullan et al., 2003).

As the employee needs to learn to develop his professional competences, many authors who mention 'professional development' as a goal also discuss the goal reflective learning ( $\mathrm{N}$ studies $=21$ ). Learning occurs more easily when reflection is stimulated. Reflection is defined by Daudelin (1996, p. 39) as: "the process of stepping back from an experience to ponder, carefully and persistently, its meaning to the self through the development of inferences; learning is the creation of meaning from past or current events that serves as a guide for future behaviour". In other words, reflection takes place if an employee uses theoretical insights to have a critical view on practice, creates insights into their own strengths and weaknesses and identifies learning needs.

The third goal, providing evidence ( $\mathrm{N}$ studies $=10$ ) refers to the employee who has to document or demonstrate his competencies (cluster $4, \mathrm{~N}$ studies $=5$ ) and is considered accountable for his own learning. The difference between cluster 3 and 4 can be explained by the intention of the employer who can use the instrument to control the formal and informal learning of the employee (cluster 3 ) or not (cluster 4).

The fifth goal, certification, selection and promotion ( $\mathrm{N}$ studies $=5$ ) refers to all kinds of different internal mobility and/or promotion, and incorporates 'receiving an award'. The goals recertification, licensure and tenure are included in this clus- 
ter as well. In contrast to the fifth cluster, the sixth cluster external mobility ( $\mathrm{N}$ studies $=4$ ) is characterized by an external selection process: the PDP is used to prepare job applications or job interviews.

The seventh cluster, coaching ( $\mathrm{N}$ studies $=1$ ) concerns stimulating and optimizing the received coaching the employee enjoys. Making use of a PDP formalizes the coaching the employee receives and subsequently improves the quality of the support. More specifically, making use of a PDP helps the coach to structure the coaching activities and prepare the coaching sessions. The employee's background, educational history and viewpoints are incorporated in the PDP and provide the coach with additional input for coaching activities (Firssova, 2006).

Cluster eight, stimulating confidence ( $N$ studies $=1$ ) refers to using the tool to boost the employee's confidence; by using a PDP, the employee becomes aware of his weaknesses and is able to undertake actions to remedy them. Finally, cluster nine, organizing ( $\mathrm{N}$ studies $=1$ ) refers to using the instrument as an agenda, to organize the learning activities an employee has already undertaken and will undertake in the future (Wildy \& Wallace, 1998).

In addition to the goals that were researched in the different studies, the goal to motivate by enhancing the employee's self-responsibility (e.g. Mathers et al., 1999) was also mentioned, but unlike the others the goal was no object of study.

As can be seen in the tables in Appendices, different goals are mentioned in the same article. The goals professional development and reflective learning are often mentioned together, as well as providing evidence; certification, selection and promotion; document or demonstrate and external mobility.

\subsection{Effectiveness}

We researched the literature on the effectiveness of PDPs as instruments to accomplish the goals they are working toward. In this context effectiveness is defined as the extent to which PDP assessment enhances the fulfilment of the goals aimed for. Only five of the references concluded that the instrument was not effective for learning, providing evidence, reflection or documenting (e.g. Orland-Barak, 2005). As shown in Table 6, the effectiveness of PDP assessment was mostly studied with regard to the following goals: personal or continuing professional development ( $\mathrm{N}$ studies $=17$ ), stimulating reflection ( $\mathrm{N}$ studies $=18$ ) and improving the professional practice or the performance ( $\mathrm{N}$ studies $=5$ ). Those clusters of goals are closely related. After all, employees need to learn by reflecting, to develop professionally and perform better. In addition to the goals for which a PDP is originally implemented (Table 5, the nine clusters), more (unintended) effects are accomplished or described, but not the object of study. These goals are also incorporated in Table 6 (see Appendices). 
In the following paragraphs we will discuss and elaborate on a couple of exemplary studies that studied the effectiveness of PDPs for professional development $(\mathrm{N}$ studies $=17)$, reflection ( $\mathrm{N}$ studies $=18$ ) and performance $(\mathrm{N}$ studies $=5$ ), the three most researched goals of PDPs. The example articles are selected because of their detailed descriptions of the research methods and the methods of analysis and/or the combination of different research methods.

Effective for professional development. (Continuing) Professional development is defined as: "the systematic maintenance, improvement, and broadening of knowledge and skills and the development of personal qualities necessary for the execution of professional and technical duties throughout the practitioner's working life" (Friedman \& Philips, 2004). Within the context of medicine it can be defined as "a process of lifelong learning for all individuals and teams which enables professionals to expand and fulfil their potential, and which also meets the needs of patients and delivers the health care priorities of the NHS" (Department of Health, 1998, p. 6, in Evans et al., 2002). Evans et al. (2002) researched the use of PDPs to stimulate the continuing professional development of General Practitioners (GPs). The main research question in this study was: Are PDPs effective for continuing professional development? Effectivity was operationalized as the perceived changes in practice and development. For this research, questionnaires were administered, measuring the demography of the GPs who use PDPs, how they identify their learning needs, what methods they use and what support they receive, their views on the use of PDPs and whether they perceive changes in their own practice and in their personal development. In addition, to explore and understand GPs' reasons for undertaking PDPs, completed PDPs from 68 GPs were analyzed. Furthermore, 19 of them were selected for semi-structured in-depth interviews to explore issues in detail. The study concludes that PDPs are effective in stimulating the continuing professional development and personal development of GPs, on the basis that it leads to changes in patient care.

Effective for reflection. By analyzing the PDPs of five medical school teachers, Tigelaar et al. (2006b) studied how a PDP stimulated reflection on the various aspects of teaching functioning. Those various aspects were based on Korthagen's model (2004) for teacher reflection in which a distinction is made between mission, identity, beliefs, competencies, behaviours and environment. Tigelaar et al. found that PDP assessment is effective for reflection. However, the research showed that this reflection is often not very profound. Teachers do not reflect easily on their motivation, feelings, thoughts and their personality. The authors therefore conclude that reflection needs to be stimulated by supportive coaches. 
Effective for performance. Wildy and Wallace (1998) conducted a study that researched whether using a PDP improves the professional knowledge and practice of school leaders $(\mathrm{N}=73$ ). The school leaders' professional knowledge and practice were operationalized as "a means of helping school leaders to understand their own accountability relationships and to account for their practice to peers" ( $p$. 124). In this study three different methods were combined, namely: PDP-analysis, surveys and observations. Evidence was found that administrators who use a PDP, develop their professional knowledge and connect it with their own practice. It was also found that the school leaders perceive the goal for which PDP assessment is used very differently, ranging from using a PDP to meet the standards to using a PDP to show the acquired competences.

Next to the goals before mentioned, a variety of other goals are studied. For an overview of those goals we refer to Table 6 in the Appendices.

\subsection{Supporting conditions}

In Table 7 (in Appendices) we give an overview of the supporting process conditions studied in the selected empirical studies. Only a limited amount of evidence is available regarding the supporting process conditions. Moreover, although supporting conditions are described in the different studies, they are not the object of study and accordingly, these studies show no empirical evidence of the role of supporting conditions in enhancing the effectiveness of PDPs. For a detailed overview of the supporting conditions that were described but not object of study, we refer to Table 8 (in the Appendices). We will focus on the supporting conditions that were the object of the research. Below we make a distinction between contextual supporting process conditions (conditions present in the working environment) and individual supporting process conditions (conditions characterizing the employee who is using the tool).

Contextual supporting process conditions. Most references indicated that the formal and fixed structure of the tool in the form of templates needs to be backed up by support. Support can be offered by different persons, namely by a coach, a mentor or a supervisor. Support can also be given by colleagues (peers). In the conversations with a coach or peer, feedback plays a central role (Tigelaar, Dolmans, Wolfhagen, \& van der Vleuten, 2004). Additionally, introductory sessions that focus on the goal, structure, use (also technical), standards and also offer guidelines can have a positive influence on how the employee makes use of a PDP. It is important to provide the employees with this information in advance. Furthermore, not only the user of a PDP needs to be supported, but the mentor or coach who offers the support needs help in his guiding role as well. To strengthen the PDP process men- 
tors need to be supported in understanding their role. They have to assist the employee in selecting and developing documentation, in linking the documentation to one or more standards and in learning from mistakes (Bradshaw \& Hawk, 1996). Finally, the learning environment that is characteristic for an organization will influence the way the employee perceives PDP assessment and will have an influence on how he uses it.

As mentioned earlier, 11 references researched the vital role of a coach in providing support. Three of these studies have been discussed in a methodologically clear way. Bullock, Firmstone, Frame and Bedward (2007) researched the implementation and impact of PDPs used by dentists. The impact of the PDP was operationalized in terms of the GP's process of development and practice. They conducted an experiment with 78 dentists, divided into an experimental group ( $N=42$ ) which was supported by a tutor in developing a PDP, and a control group ( $N=45)$. The researchers found that dentists who received support from a tutor undertook more courses (23\% versus $18 \%$ ), discussions with colleagues and trainings (43\% versus 32\%). Furthermore, the experimental group selected learning activities more in tune with their learning needs (40\% versus $32 \%$ ) and reported a higher impact of the learning activities ( $21 \%$ versus $15 \%$ ). The research also highlighted that the influence of the coach's support is stronger in case of informal activities than formal activities. This can be explained by the fact that a PDP provides the dentist with a certain focus while he reads and hereby enlarges the educational benefits.

By taking questionnaires analyzing the PDP construction process and taking semi-structured in depth-interviews, Firssova (2006) found that making use of a PDP also enlarges the coaching repertoire and the perceived efficiency and effectiveness of coaching. "The PDP helped to structure the coaching activities, supported preparation of the coach for coaching sessions and served as an additional feedback channel. The coach got broader access to the general background, educational history and viewpoints of the PDP-maker which provided additional input for coaching activities" (Firssova, 2006, p. 11).

Research by Snadden and Thomas (1998) indicated that if confidence is low and the relationship with the trainer is good, the GP is most likely to use the PDPinstrument effectively. The researchers conducted semi-structured interviews to come to that conclusion. In relation to confidence the authors stated: "Confidence was low on entering practice and was one of the factors that acted in a positive way to encourage the adoption of the PDP ... In addition to this trust, facilitation of the exploration of difficult areas and the generation of a curriculum based on experience between both GP and trainer was required" (Snadden \& Thomas, 1998, p. 404).

Four references discussed the role of peers as a possible source of support and how they could contribute to the professional practice of their colleagues, for example because of the possibility to integrate different viewpoints. Moore and Bond 
(2002) and Tigelaar et al. (2006) explicitly studied the role of social interactions with peers and found positive results by taking interviews and, in the case of Tigelaar et al., also analyzing PDPs. The latter authors state: "Most of the teachers found the peer meetings valuable because sharing experiences, hearing different viewpoints, thinking along with others and receiving feedback made them take a fresh look at their own teaching and, in some cases, stimulated them to improve practice" (Tigelaar et al., 2006, p. 375). Often the term 'collaboration' is used to point out the interactions with the mentor and/or colleagues, also called peer learning/sharing or collegiality (Seng \& Seng, 1996).

The feedback that is offered during the meetings with the coach/supervisor or colleagues plays an important role. Four references focused on the feedback that is provided as a result of using a PDP, which was found to be a positive and essential part of the process for growth and professional development (e.g. Smith \& Tillema, 1998/2003). Combining PDP analysis, semi-structured questionnaires and in-depth interviews, Smith and Tillema (2003) found that in using a dossier, training and reflective PDP, external feedback is valued the most when it is given by a superior.

Furthermore training lessons ( $\mathrm{N}$ studies $=1$ ) and providing technical support ( $\mathrm{N}$ studies $=4$ ) were found to have a positive effect; although in most cases there were not enough resources to provide training on a regular basis (e.g. Dornan, Carroll, \& Parboosingh, 2002).

Also seen as a supporting condition is the assessment structure that is offered by the organization or the supervisor ( $N$ studies $=2$ ). It is found that when the employees are provided with a clear goal, a formal framework (including meetings), specific guidelines and standards, the effects of PDP assessment will be larger (e.g. Guaglianone, 1995).

Next, three references highlighted that a supportive learning environment is found to be stimulating. Johnston and Thomas (2005) found that principals perceived the PDP as a tool for professional development if it was situated within a larger supportive social network of professional practice. By contrast, if there was hardly any sense of community, PDP assessment was not stimulating the development of the practice.

Finally, the effectiveness of PDP assessment depends on the available resources ( $\mathrm{N}$ studies $=3$ ), the time provided ( $\mathrm{N}$ studies $=7$ ) and on whether the use is voluntary or mandatory ( $\mathrm{N}$ studies $=1$ ). Research indicates that PDP assessment that is voluntary has more effect on the professional development of the individual (Beck et al., 2005; Smith \& Tillema, 2001; Swallow, Clarke, lles, \& Harden, 2006). Nevertheless, the question remains: What do you do with less motivated employees? (Swallow et al., 2006). For example, Bunker and Leggett (2004) found that staff did not develop a PDP unless they were obliged to. 
Individual supporting process conditions. Next to the above mentioned supporting conditions that are part of the organizational structure, a limited amount of articles ( $\mathrm{N}$ studies $=16$ ) distinguished a group of personal characteristics that influence the effectiveness of the instrument. Austin et al. (2005, p. 181) even describes the PDP as an instrument that requires: "an idealized type of individual who knows how to self-reflect, is open to change, interested in his own development and knows how to organize himself and his environment to support learning (self-directedness, autonomy)". By conducting an action-research in post-graduate nursing practice Joyce (2005) found that confidentiality in committing experiences to paper is an issue when using a PDP. Dornan et al. (2002) questioned 439 physicians. They found support for reflective learning using an e-PDP. Acceptability and use were influenced by the physicians' individual learning style.

Smith and Tillema (2003) found the perceived feasibility - i.e. whether or not an individual can produce the required information - to be one of the major causes for differences in PDP use. The quantitative and qualitative research they conducted with 89 teachers showed that the PDP type that gave space and opportunity for self-directed learning was regarded as more feasible than the other PDP types. Other researchers indicated that a lack of motivation can cause difficulties in composing a PDP (Bahrami, Rogers, \& Singleton, 1995). To study this, they questioned 40 GPs. Lastly, Smith and Tillema (1998) concluded that high quality PDPs can only be expected after sustained use. They executed a study with principals ( $N$ studies $=35$ ) and a study with managers ( $\mathrm{N}$ studies $=14$ ) by taking questionnaires and interviews. The studies focused on the use of a PDP as a tool for providing functional feedback.

Furthermore, the importance of possessing writing skills (Cayne, 1995), being flexible (e.g. Evans et al., 2002), believing in the process (e.g. Pearson \& Heywood, 2004) and having confidence/trust in the environment (e.g. Pitts, Curtis, While, Holloway, 1999) are believed to be valuable characteristics of the individual. Also, when the PDP assessment is in line with the employee's learning needs and when there is a clear connection with the day-to-day practice, the perceived benefit will lead towards an increased enthusiasm and personal satisfaction, which will in turn stimulate personal development (Austin et al., 2005; Bahrami et al., 1995; Bullock et al., 2007). 
Chapter 2

Table 3

Integrated table: Goals, effects and supporting process conditions

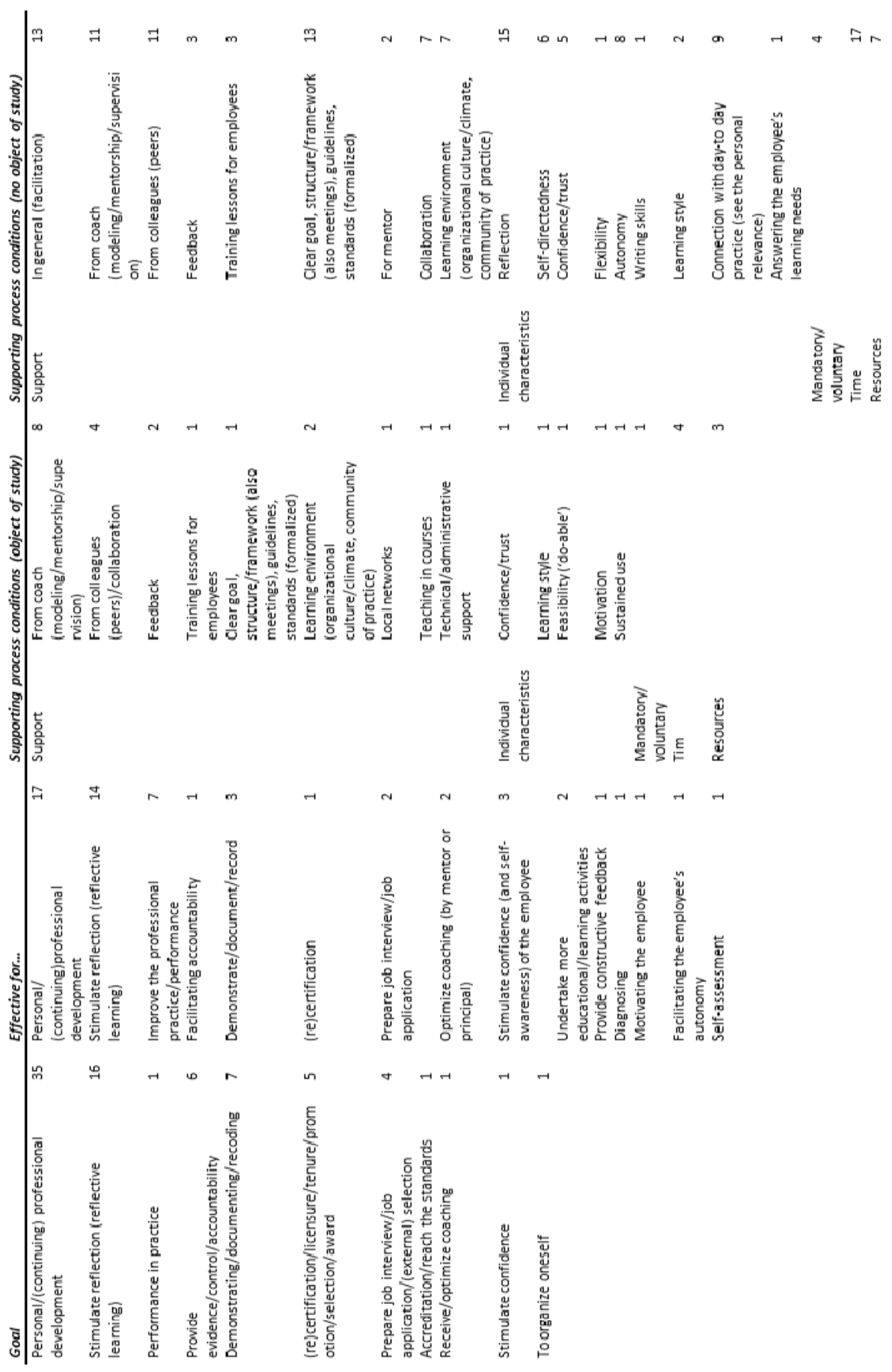




\section{Conclusion and discussion}

Nowadays employability and talent management of workers is taking a central role in organizations, as companies come to realize that the talent and competencies of the employees drive their business success (Guthridge et al., 2008). To develop those talents and competencies, organizations to a growing extent make use of PDPs as an assessment tool, providing and documenting information on the competencies the employee worked on and is planning to further develop. The purpose of this paper was to explore the literature on the goals PDPs are being used for in the workplace (research question one), on their effectiveness (research question two) and on the conditions which can support their effectiveness (research question three). To this end we conducted a literature review to examine the three research questions. To our knowledge, this is the first systematic review of the empirical literature on PDP assessment in the workplace. As most literature on the topic is descriptive, only 54 relevant empirical studies were included. Furthermore, most studies lacked a detailed description of the research method or data analysis.

Concerning the first research question: For which goals is PDP assessment used? We stated that in general a distinction is made between professional development and certification/selection/accountability (Smith \& Tillema, 2001). Based on literature in this study nine clusters of goals were identified, namely: (1) professional development; (2) reflective learning; (3) providing evidence; (4) documenting; (5) certification, selection and promotion; (6) external mobility; (7) coaching; (8) stimulating confidence; and (9) organizing.

In addition, some authors categorize the aforementioned goals of PDPs according to the purpose of assessment. A distinction is made between the formative and summative purposes of PDP assessment. While some authors state that formative and summative assessment has a different audience, namely colleagues (learning) versus the organization (assessment) (e.g. Wolf \& Dietz, 1998), other authors believe that information gathered during the learning or development process can be very useful for summative evaluation (e.g. Snyder, Pippincott \& Bower, 1998).

Answering the second research question, the effectiveness of PDP assessment, the results of the empirical studies are inconclusive. Some studies indicate that PDP assessment is effective for personal or continuing professional development, stimulating reflection and improving professional practice. However, not all studies support these positive findings. For example, Bunker and Leggett (2005) found that (teaching) PDPs can be effective tools for stimulating promotion, but they are not useful for encouraging reflective practice. These inconclusive results indicate that the effectiveness of PDP assessment is depending on certain supporting process conditions. This brings us to the third research question: Which supporting process conditions enhance the effectiveness of PDP assessment? The literature indicated that the use of PDPs needs to be backed up by sufficient support. Support can be 
offered by different key players, such as a coach, a mentor, a supervisor or a colleague. During conversations with a coach or a colleague, the feedback given plays a crucial role. Additionally, introductory sessions that focus on the goal, structure, use (also technical) and assessment criteria have a positive influence on how the employee makes use of a PDP. It is important to provide the employees with this information in advance. However, the tool should not be too highly organized either because that would prevent the employee from taking the responsibility and personalizing the PDP (Tigelaar et al., 2004). Not only the user of a PDP needs support; the mentor or coach who offers the support needs help in his guiding role as well (e.g. Snyder et al., 1998). To strengthen the PDP process mentors need to be supported in understanding their role. They must assist in selecting and developing documentation, in linking the documentation to one or more standards and in learning from mistakes. Finally, the learning environment that is characteristic for an organization will influence the way the employee perceives PDP assessment and will have an influence on how they use it.

Next to contextual supporting process conditions, several individual supporting process conditions are mentioned in the literature. The idealized type of user is an employee who is motivated and dedicated to his own development, knows how to self-reflect, shows self-directedness and has confidence in his supervisor and the assessment process. However, organizations and supervisors often do not provide the employee with sufficient time to reflect and work on his development plan (Alsop, 2002).

In conclusion, implementing PDP assessment in the organization should not be seen as bringing in a Trojan horse, a tool that is brought in with high expectations but appears to contain unpleasant surprises only. If supporting process conditions are present; such as the support from a coach, the effectiveness of the PDP as an assessment tool to support development, is enhanced.

Nevertheless, there exists a gap when it comes to empirical research on the effectiveness of PDP use in the workplace and the conditions that can support this use, especially in organizational contexts other than educational and health settings. For example, the received support from a coach was the only supporting process condition that was an object of study in more than four studies. The lack of empirical research on this topic may be explained by the fact that PDP assessment has only been implemented in organizations in the course of the last decennium. This is especially the case if the instrument is used for other purposes than evaluation purposes. The small amount of empirical research that is available is mostly qualitative research, with small sample sizes.

This literature review encountered a few limitations that need to be brought into account. First, the search showed that empirical evidence of the effectiveness of PDPs to reach the goals aimed as well as of the necessary supporting conditions, is still scarce. We were able to select 57 references of which three could not be 
found. Moreover, the methodology is often poorly described in the studies and, if the studies are quantitative, the presented results are mainly limited to frequencies and means. Statistical meta-analysis evidencing effects sizes therefore could not yet be performed.

Second, the concept of PDP has many connotations in literature as well as practice. Although in order to enhance comparability of the literature reviewed, only articles where the description of the PDP matches the characteristics we have defined, were included, there are still variations in the implementation of this tool. Given the limited amount of studies, those differences were not taken into account and further analysis to improve differences between organizational implementation and practice were not possible.

This study clearly shows that empirical research on this topic is scarce. Therefore future empirical research should focus on the central question reviewed in this study: Is PDP assessment effective and if so, for which goals and under which conditions? Firstly, there has been no systematic empirical research on the goals PDP assessment is used for. It is not clear for which various goals PDP assessment is used in practice. Next, it can be questioned if the PDP is suitable for all kinds of goals and in which way the tool should be used differently according to the goal it is used for. For example, the role and the task of the supervisor will be different when a PDP is used for a summative purpose (e.g. promotion) than when a PDP is used to accomplish a formative purpose (e.g. professional development). It was also found that the PDP is effective for reflective learning.

Secondly, there is a need for more empirical research on the effectiveness of PDP assessment in organizational settings, other than educational and health contexts. More concretely, from a methodological point of view, studies with research designs that allow the measurement of effects such as pre-test post-test control group designs, need to be conducted. However, identifying organizations that consist of a group of users and non-users of PDPs is not always possible and assessment processes in which the tool takes a central role may vary a lot between different organizations; which puts the external validity under pressure. In addition, to be able to measure the effects of the PDP practice, there is a need for validated instruments. Future research should research the components that are part of the PDP practice (e.g. the format, the assessment process, the supervisor and the delivered feedback) and focus on developing and validating instruments that measure the PDP practice and use. This includes the challenge to get a grip on the different components that are part of the PDP practice. Furthermore, the issue of measuring the effectiveness of a tool like a PDP includes the challenge of operationalizing the construct of effectiveness. Many discussions have been held about how to define effects of professional development interventions. Often is referred to the fourlevels model of Kirkpatrick in which a distinction is made between four levels of evaluation: reactions, learning, behavior and results (Aguinis \& Kraiger, 2009). The 
effectiveness of a PDP, defined as a learning and development tool, can be measured on the same four levels. Is it possible to measure the effects of a PDP in terms of the emplyees' satisfaction (level 1: reaction); the effects on learning activities undertaken by the employee (level 2: learning), expertise growth (level 3: behavior) and performance of the employee (level 4)? Finally, since a PDP is mostly used in order to stimulate learning and development over a longer period of time, longitudinal research could specify the long-term effects of the assessment tool. It may be possible that PDPs are only effective if they are used in a sustainable way.

Thirdly, to provide organizations with guidelines about how to organize the PDP assessment process and consequently make PDP assessment more effective, more research on the contextual and individual supporting process conditions of effective PDP assessment in organizational contexts is needed. While the PDP is claimed to be a tool to enhance learning, it often seems to have the opposite effect. Consequently, questions arise concerning the factors that influence the use of a PDP in a positive and/or a negative way, leading to reflective learning and continuing professional development. This leads to a number of specific questions concerning the contextual supporting process conditions discussed in this literature review that future research could address, such as: How does the PDP assessment process need to be supported? What does a supervisor need to do? How many formal meetings should take place to discuss the PDP? How does the supervisor need to give feedback on the PDP? How can the supervisor motivate the employee to make use of a PDP?

With respect to the individual supporting process conditions, relevant questions for future research are: Is PDP assessment applicable to every type of employee? Is it applicable to knowledge workers and non-knowledge workers, in profit and non-profit organizations? What skills does an employee need to possess to be able to use a PDP in an effective way? Can an employee be trained in using a PDP?

In conclusion, the results of our study provide more insight into the use of PDP assessment in the workplace. However, more studies systematically investigating the effects of PDPs and the supporting process conditions are necessary. 


\section{References}

Aguinis, H., \& Kraiger, K. (2009). Benefits of training and development for individuals and teams, organizations, and society. Annual Review of Psychology, 60, 451-474.

Alsop, A. (2002). Portfolios: Portraits of our professional lives. British Journal of Occupational Therapy, 65 (5), 201-206.

Austin, Z., Marini, A., \& Desroches, B. (2005). Use of a learning portfolio for continuous professional development: A study of pharmacists in Ontario (Canada). Pharmacy Education, 5, 175-181.

Bahrami, J., Rogers, M., \& Singleton, C. (1995). Personal education plan: A system of continuing medical education for general practitioners. Education for General Practice, 6, 342-345.

Beck, R.J., Livne, N.L., \& Bear, S.L. (2005). Teachers' self-assessment of the effects of formative and summative electronic portfolios on professional development. European Journal of Teacher Education, 28, 221-244.

Boud, D., Keogh, R., \& Walker, D. (Eds.) (1985). Reflection: Turning experience into learning. London: Kogan Page.

Bradshaw, L.K., \& Hawk, P.P. (1996). Portfolios for the continuing licensure of beginning teachers: Their development and assessment. Paper presented at the Annual Meeting of the National Staff Development Council (Vancouver, British Columbia, Canada, December 10, 1996).

Brown, R. (1995). Portfolio development and profiling for nurses, $2^{\text {nd }}$ edn. Lancaster: Quay Publications.

Brown, G., \& Irby, B.J. (1998). Recent developments in the use of career advancement portfolios for administrators: Implications for leadership preparation programs. Paper presented at the Annual Meeting of the American Educational Research Association (San Diego, CA, April 13-17, 1998).

Bullock, A., Firmstone, V., Frame, J., \& Bedward, J. (2007). Enhancing the benefit of continuing professional development: A randomized controlled study of personal development plans for dentists. Learning in Health and Social Care, 6 (1), 14-26.

Bunker, A., \& Leggett, M. (2004). Being wise about teaching portfolios: Exploring the barriers to their development and maintenance. Retrieved from http://www.herdsa.org.au/wp-content/uploads/ conference/2004/PDF/P047-jt.pdf on 20/09/2007.

Bunker, A. \& Leggett, M. (2005). Teaching portfolios: rhetoric, reality and reflection. (unpublished).

Challis, M. (2001). Portfolios and assessment: Meeting the challenge. Medical Teacher, 23, 437-440.

Cayne, J.V. (1995). Portfolios: A developmental influence? Journal of Advanced Nursing, 21, 395-405.

CBI (Confederation of British Industry) (1999). Making employability work: An agenda for action. London: $\mathrm{CBI}$.

Daniel, P.L., \& Stallion, B.K. (1995). Research report on the implementation of professional development in Kentucky. Paper presented at the Annual Meeting of the Mid-South Educational Research Association (Biloxi, MS, November 9, 1995).

Daudelin, M. W. (1996). Learning from experience through reflection. Organizational Dynamics, 24 (3), 36-48.

Dixon, R., Dixon, K., \& Pelliccione, L. (2005). The professional electronic portfolio project: The production process. Retrieved from http://www.ascilite.org.au/conferences/brisbane05/blogs/proceedings /19_Dixon.pdf on 20/09/2007.

Dornan, T., Carroll, C., \& Parboosingh, J. (2002). An electronic learning portfolio for reflective continuing professional development. Medical Education, 36, 767-769.

Driessen, E.W., van Tartwijk, J., van der Vleuten, C.P.M., \& Wass, V.J. (2007). Portfolios in medical education: Why do they meet with mixed success? A systematic review. Medical Education, 41, 12241233.

Ekbatani, G., \& Pierson, H. (1997). Teacher portfolios, vehicles of faculty assessment, reflection, and growth. Paper presented at the Annual Meeting of the Teachers of English to Speakers of Other Languages (31st, Orlando, FL, March, 11-15, 1997). 
Evans, A., Ali, S., Singleton, C., Nolan, P., \& Bahrami, J. (2002). The effectiveness of personal education plans in CPD: An evaluation. Medical Teacher, 24 (1), 79-84.

Fenwick, T. J. (2003). Professional growth plans: Possibilities and limitations of an organizationwide employee development strategy. Human Resource Development Quarterly, 14, 59-77.

Firssova O. (2006). ePortfolio as a coaching support tool for workplace learning of teachers. Paper presented at ePortfolio conference October 11-13, 2006, Oxford. Retrieved from: http://www.eifel.org/news/ep2006/programme on 20/09/2007.

Friedman, A, \& Philips, M. (2004). Continuing professional development: Developing a vision. Journal of Education and Work, 17, 361-376.

Grandgenette, R. S. (1999). The professional development 2001 Portfolio. Journal of the American Dietetic Association, 99 (5), 612-614.

Guaglianone, C.L. (1995). Portfolio assessment of administrators. Paper presented at the Annual Meeting of the National Council of Professors of Educational Administration, $49^{\text {th }}$, Williamsburg, VA, August 8-12, 1995.

Guaglianone, C.L., \& Yerkes, D.M. (1998). Administrative portfolios: Current uses and development by prospective and practicing administrators. Paper presented at the Annual Meeting of the American Educational Research Association

Guthridge, M., Komm, A.B., \& Lawson, E. (2008). Making talent a strategic priority. The McKinsey Quarterly, No. 1.

Hart, C. (1998). Doing a literature review. Releasing the social science research imagination. London: Sage Publications.

Hrisos, S., Illing, J.C., \& Burford, B.C. (2008). Portfolio learning for foundation doctors: Early feedback on its use in the clinical workplace. Medical Education, 42, 214-223.

Irby, B.J., \& Brown, G. (1999). A gendered dichotomy in written reflections in professional development portfolios. Paper presented at the Annual Meeting of the American Educational Research Association (Montreal, Quebec, Canada, April 19-23, 1999)

Johnston, M., \& Thomas, M. (2005). Riding the wave of administrator accountability: A portfolio approach. Journal of Educational Administration, 43 (4), 368-386.

Joo, B.-K. (2005). Executive Coaching: A Conceptual Framework From an Integrative Review of Practice and Research. Human Resource Development Review, 4, 462-488.

Joyce, P. (2005). A framework for portfolio development in postgraduate nursing practice. Journal of Clinical Nursing, 14, 456-463.

Jun, M.-K., Anthony, R., Achrazoglou, J., \& Coghill-Behrends, W. (2007). Using eportfolio for the assessment and professional development of newly hired teachers. Techtrends, 51 (4), 45-50.

Kicken, W., Brand-Gruwel, S., van Merriënboer, J., Slot, W. (2008). Design and evaluation of a development portfolio: How to improve students' self-directed learning skills. Instructional Science, 37, 453-473.

Kjaer, N.K., Maagaard, R., \& Wied, S. (2006).Using an online portfolio in postgraduate training. Medical Teacher, 28, 708-712.

Knowles, M. (1975). Self-directed learning: A guide for learners and teachers. Chicago: Follet.

Kolb, D.A. (1984). Experiential learning. Chicago: Prentice Hall.

Korthagen, F.A.J. (2004). In search of the essence of a good teacher: Towards a more holistic approach in teacher education. Teaching and Teacher Education, 20, 77-97.

Lammintakanen, J., Saranto, K., Kivinen, T., \& Kinnunen, J. (2002). The digital portfolio: A tool for human resource management in health care? Journal of Nursing Management, 10, 321-328.

Leggett, M., \& Bunker, A. (2006). Teaching portfolios and university culture. Journal of Further and Higher Education, 30, 269-282.

Little, P., \& Hayes, S. (2003). Continuing professional development (CPD): GPs' perceptions of postgraduate education-approved (PGEA) meetings and personal professional development plans (PDPs). Family Practice, 20 (2), 192-198. 
London, M., Larsen, H. H., \& Thisted, L. N. (1999). Relationships between feedback and selfdevelopment. Group \& Organizational Management, 24, 5-27.

London, M., \& Smither, J.W. (1999). Empowered self-development and continuous learning. Human Resource Management, 38, 3-15.

Lyons, N., \& Evans, L. (1997). Portfolio: A tool for self-directed learning at work. Paper presented at the Self-directed Learning: Past and Future Symposium (Montreal, Quebec, Canada, September 1997).

Lyons, N. (1998). Reflection in teaching: Can it be developmental? A portfolio perspective. Teacher Education Quarterly 25, 115-127.

Marcoux, J., Brown, G., Irby, B. J., Lara-Alecio, R. (2003). A case study on the use of portfolios in principal evaluation. Paper presented at the Annual Meeting of the American Educational Research Association, Chicago, IL, (21-25 April 2003)

Mathers, N.J., Challis, M.C., Howe, A.C., \& Field, N.J. (1999). Portfolios in continuing medical education effective and efficient? Medical Education, 33, 521-530.

McMullan, M., Endacott, R., Gray, M.A., Jasper, M., Miller, C.M.L., Scholes, J., \& Webb, C. (2003). Portfolios and assessment of competence: A review of the literature. Journal of Advanced Nursing, 41, 283-294.

Moore, Z., \& Bond, N. (2002). The use of portfolios for in-service teacher assessment: A case study of foreign language middle-school teachers in Texas. Foreign Language Annals, 35 (1), 85-92.

Noe, R. A. (1988). An investigation of the determinants of successful assigned mentoring relationships. Personnel Psychology, 41, 457-479.

Noe, R. A. (1996). Is Career Management Related to Employee Development and Performance? Journal of Organizational Behavior, 17, 119-133.

O'Connor, C.R., \& Herrelko, J. (2000). Individual professional development plans: In search of learning for teaching. Paper presented at the Mid-Western Educational Research Association's Annual Conference (27 October 2000).

Orland-Barak, L. (2005). Portfolios as evidence of reflective practice: What remains "untold". Educational Research, 47 (1), 25-44.

Pearson, D.J., \& Heywood, P. (2004). Portfolio use in general practice vocational training: A survey of GP registrars. Medical Education, 38, 87-95.

Pinder, H., \& Turnbull, M. (2003). Teacher professional development; A portfolio approach. Paper presented at the British Educational Research Association Annual Conference, Heriot-Watt University, Edinburgh, 11-13 September 2003.

Pitts, J., Curtis, A., While, R., Holloway, I. (1999). Practice professional development plans: General practitioners' perspectives on proposed changes in general practice education. British Journal of General Practice, 49, 959-962.

Redman, W. (1994). Portfolios for development: A guide for trainers and managers. London: Kogan Page.

Riggs, I.M., Sandlin, R.A., Scott, L.D., Childress, L., \& Mitchell, D.E. (1997). The new teacher portfolio: $A$ bridge to professional development. Paper presented at the Annual Meeting of the American Educational Research Association. Chicago, IL, 24-28 March 1997.

Roberts, G. E. (2003). Employee Performance Appraisal System Participation: A Technique that Works. Public Personnel Management, 32, 89-98.

Schmitz, C.C., \& Schillo, B.A. (2005). Report Carding: A model for foundation portfolio assessment. Americain Journal of Evaluation, 26, 518-531.

Seng, S.H., \& Seng, T.O. (1996). Reflective teaching and the portfolio approach in early childhood staff development. Paper presented at the Joint Conference of the Educational Research Association of Singapore and the Australian Association for Research in Education. Singapore, 25-29 November 1996.

Smith, K., \& Tillema, H. (1998). Evaluating portfolio use as a learning tool for professionals. Scandinavian Journal of Educational Research, 42, 193-205.

Smith, K., \& Tillema, H. (2001). Long-term influences of portfolios on professional development. Scandinavian Journal of Educational Research, 45, 183-202. 
Smith, K., \& Tillema, H. (2003). Clarifying different types of portfolio use. Assessment \& Evaluation in Higher Education, 28, 625-648.

Snadden, D., \& Thomas, M.L. (1998). Portfolio learning in general practice vocational training does it work? Medical Education, 32, 401-406.

Snadden, D., Thomas M.L., Griffin, E.M., \& Hudson, H. (1996). Portfolio-based learning and general practice vocational training. Medical Education, 30, 148-152.

Snyder, J., Pippincott, A., \& Bower, D. (1998). The inherent tensions in the multiple use of portfolios in teacher education. Teacher Education Quarterly, 25 (1), 45-60.

Spence, W., El-Ansari, W. (2004). Portfolio assessment: practice teacher's early experience. Nurse Education Today, 24, 388-401.

Stewart, I. (2004). Using portfolios to improve teaching quality: The case of a small business school. Journal of Education for business, 80 (2), 75-79.

Stone, B.A. (1998). Problems, pitfalls, and benefits of portfolios. Teacher Education Quarterly, 25 (1), 105-114.

Sullivan, J.H. (2004). Identifying the best foreign language teachers: Teacher standards and professional portfolios. The Modern Language Journal, 88, 390-402.

Swallow, V., Clarke, C., lles, S., \& Harden, J. (2006). Work based, lifelong learning through professional portfolios: Challenge or reward? Pharmacy Education, 6 (2), 77-89.

Tigelaar, D.E.H., Dolmans, D.H.J.M., de Grave, W.S., Wolfhagen, I.H.A.P., van der Vleuten, C.P.M. (2006). Participants' opinions on the usefulness of a teaching portfolio. Medical Education, 40, 371-378.

Tigelaar, D.E.H., Dolmans, D.H.J.M., Wolfhagen, I.H.A.P., \& van der Vleuten, C.P.M. (2004). Using a conceptual framework and the opinions of portfolio experts to develop a teaching portfolio prototype. Studies in Educational Evaluation, 30, 305-321.

Tigelaar, D.E.H., Dolmans, D.H.J.M., De Grave, W.S., Wolfhagen, I.H.A.P., \& Van der Vleuten, C.P.M. (2006b). Portfolio as a tool to stimulate teachers' reflections. Medical Teacher, 28, 277-282.

Tillema, H.H. (2001). Portfolios as developmental assessment tools. International Journal of Training and Development, 5 (2), 126-135.

Tillema, H.H. (1998). Design and validity of a portfolio instrument for professional training. Studies in Educational Evaluation, 24, 263-278.

Tillema, H., \& Smith, K. (2000). Learning from portfolios; differential use of feedback in portfolio construction. Studies in Educational Evaluation, 26, 193-210.

Tisani, N. (2008). Challenges in producing a portfolio for assessment: In search of underpinning educational theories. Teaching in Higher Education, 13, 549-557.

Tucker, P.D., Stronge, J.H., Gareis, C.R., \& Beers, C.S. (2003). The efficacy of portfolios for teacher evaluation and professional development: Do they make a difference? Educational Administration Quarterly, 39, 572-602.

Van der Schaaf, M., Stokking, K., Verloop, N. (2005). Cognitive representations in raters' assessment of teacher portfolios. Studies in Educational Evaluation, 31, 27-55.

Wasylyshyn, K. M. (2003). Executive Coaching: an outcome study. Consulting Psychology Journal: Practice and Research, 55, 94-106.

Webb, T.P., Aprahamian, C., Weigelt, J.A., \& Brasel, K.J. (2006). The surgical learning and instructional portfolio (SLIP) as a self-assessment educational tool demonstrating practice-based learning. Current Surgery, 63, 444-447.

Wildy, H., \& Wallace, J. (1998). Professionalism, portfolios and the development of school leaders. School Leadership and Management, 18 (1), 123-140.

Wolf, K., \& Dietz, M. (1998). Teaching portfolios: Purposes and possibilities. Teacher Educational Quarterly, 25 (1), 9-22.

Wright, W. A., Knight, P. T., \& Pomerleau, N. (1999). Portfolio people: Teaching and learning dossiers and the future of higher education. Innovative Higher Education, 24 (2), 89-102.

Zeichner, K., \& Wray, S. (2001). The teaching portfolio in US teacher education programs: what we know and what we need to know. Teaching and teacher education, 17, 613-621. 


\section{Appendices}

Table 4

Number of references according to the research method

Number of

references

Research

1. Quantitative research

- Survey/questionnaire

Austin, Marini, \& Desroches, 2005; Dixon,

35 unique hits

Dixon, \& Pelliccione, 2005; Grandgenette, 1999;

Guaglianone, 1995; Guaglianone \& Yerkes, 1998; Hrisos, Illing, \& Burford, 2008; Johnston

\& Thomas, 2005; Jun, Anthony, Achrazoglou, \& Coghill-Behrends, 2007; Seng \& Seng, 1996;

Tisani, 2008; Tucker, Stronge, Gareis, \& Beers, 2003; Webb, Aprahamian, Weigelt, \& Brasel, 2006; Wildy \& Wallace, 1998.

- Questionnaire

Bahrami, Rogers, \& Singleton, 1995; Beck, Livne, \& Bear, 2005; Bunker \& Leggett, 2004; Cayne, 1995; Dornan, Carroll, \& Parboosingh, 2002; Evans, Shahid, Singleton, Nolan, \& Bahrami, 2002; Firssova, 2006, Joyce, 2005; Kjaer, Maagaard, Wied, 2006; Lammintakanen, Saranto, Kivinen, \& Kinnunen, 2002; Little \& Hayes, 2003; Marcoux, Brown, Irby, \& Lara-Alecio, 2003; Mathers, Challis, Howe, \& Field, 1999; Pearson \& Heywood, 2004; Smith \& Tillema, 1998/2001/2003/2007; Spence \& El-Ansari, 2004; Sullivan, 2004; Tillema, 2001/1998.

- Experiment

2.Qualitative research

- Interviews
Ekbatani \& Pierson, 1997.

Bradshaw \& Hawk, 1996; Brown \& Irby, 1998; Bullock, Firmstone, Frame, \& Bedward, 2007; Cayne, 1995; Daniel \& Stallion, 1995; Dixon, Dixon, \& Pelliccione, 2005; Evans, Shahid, Singleton, Nolan, \& Bahrami, 2002; Fenwick, 2003; Firssova, 2006; Hrisos, Illing, \& Burford, 2008; Johnston \& Thomas, 2005; Lyons, 1998; Marcoux, Brown, Irby, \& Lara-Alecio, 2003; Mathers, Challis, Howe, \& Field, 1999; Pearson \& Heywood, 2004; Pitts, Curtis, While, \& Holloway, 1999; Smith \& Tillema, 1998//2001/2003/2007; Snadden \& Thomas, 1998; Snadden, Thomas, Griffin, \& Hudson, 1996; Sullivan, 2004; Swallow, Clarke, lles, \& Harden, 2006; Tigelaar, Dolmans, de Grave, Wolfhagen, \& van der Vleuten, 2006; Tisani, 2008. 


\begin{tabular}{|c|c|c|}
\hline - Focus groups & $\begin{array}{l}\text { Bunker \& Leggett, 2004; Johnston \& Thomas, } \\
\text { 2005; Joyce, 2005; Marcoux, Brown, Irby, \& } \\
\text { Lara-Alecio, 2003; Tucker, Stronge, Gareis, \& } \\
\text { Beers, 2003. }\end{array}$ & 5 \\
\hline - Participant observation & $\begin{array}{l}\text { Mathers, Challis, Howe, \& Field, 1999; Stewart, } \\
\text { 2004; Wildy \& Wallace, } 1998 .\end{array}$ & 3 \\
\hline - Portfolio analysis & $\begin{array}{l}\text { Bullock, Firmstone, Frame, \& Bedward, 2007; } \\
\text { Dixon, Dixon, \& Pelliccione, 2005; Evans, Sha- } \\
\text { hid, Singleton, Nolan, \& Bahrami, 2002; Firsso- } \\
\text { va, 2006, Irby \& Brown, 1999; Johnston \& } \\
\text { Thomas, 2005; Mathers, Challis, Howe, \& Field, } \\
\text { 1999; Moore \& Bond, 2002; O’ Connor \& Her- } \\
\text { relko, 2000; Orland-Barak, 2005; Pinder \& } \\
\text { Turnbull, 2003; Riggs, Sandlin, Scott, Childress, } \\
\text { \& Mitchell, 1997; Smith \& Tillema, 1998; Sulli- } \\
\text { van, 2004; Tigelaar et al., 2006b; Wildy \& } \\
\text { Wallace, 1998. }\end{array}$ & 16 \\
\hline - Anecdotal feedback & $\begin{array}{l}\text { Austin, Marini, \& Desroches, 2005; Dornan, } \\
\text { Carroll, \& Parboosingh, } 2002 .\end{array}$ & 2 \\
\hline - (semi-structured) & Austin, Marini, \& Desroches, 2005; Bunker \& & 3 \\
\hline Discussion session /workshops & Leggett, 2004; Cayne, 1995. & \\
\hline - Case analysis & Beck, Livne, \& Bear, 2005; Lyons \& Evans, 1997. & 2 \\
\hline - Field notes & Cayne, 1995. & 1 \\
\hline - Meetings & Dixon, Dixon, \& Pelliccione, 2005. & 1 \\
\hline
\end{tabular}

Table 5

Studies sorted according to the number of participants

\begin{tabular}{lr}
\hline Number of participants & Number of references \\
\hline $0<N>50$ & 37 \\
$N<10$ & 7 \\
$10<N>20$ & 11 \\
$20<N>30$ & 7 \\
$30<N>40$ & 9 \\
$40<N>50$ & 3 \\
$50<N>100$ & 11 \\
$N>100$ & 14 \\
$100<N>200$ & 6 \\
$200<N>500$ & 5 \\
$N>500$ & 3 \\
\hline
\end{tabular}


Table 6

PDP Assessment in the workplace: Which goals are being strived for?

\begin{tabular}{|c|c|}
\hline Goal $^{9}$ & References \\
\hline $\begin{array}{l}\text { Personal/(continuing) profes- } \\
\text { sional development }(42)\end{array}$ & $\begin{array}{l}\text { Austin, Marini, \& Desroches, 2005; Bahrami, Rogers, \& Singleton, } \\
\text { 1995; Beck, Livne, \& Bear, 2005; Bullock, Firmstone, Frame, \& } \\
\text { Bedward, 2007; Cayne, 1995; Daniel \& Stallion, 1995; Dixon, Dixon, } \\
\text { \& Pelliccione, 2005; Dornan, Carroll, \& Parboosingh, 2002; Ekbatani } \\
\text { \& Pierson, 1997; Evans, Ali, Singleton, Nolan, \& Bahrami, 2002.; } \\
\text { Fenwick, 2003; Firssova, 2006; Grandgenette, 1999; Hrisos, Illing, \& } \\
\text { Burford, 2008; Irby \& Brown, 1999; Johnston \& Thomas, 2005; } \\
\text { Joyce, 2005; Jun, Anthony, Achrazoglou, \& Coghill-Behrends, 2007; } \\
\text { Lammintakanen, Saranto, Kivinen, \& Kinnunen, 2002; Little \& } \\
\text { Hayes, 2003; Lyons \& Evans, 1997; Marcoux, Brown, Irby \& Lara- } \\
\text { Alecio, 2003; Mathers, Challis, Howe, \& Field, 1999; Moore \& Bond, } \\
\text { 2002; O’Connor \& Herrelko, 2000; Pinder \& Turnbull, 2003; Pitts, } \\
\text { Curtis, While, \& Holloway, 1999; Riggs, Sandlin, Scott, Childress, \& } \\
\text { Mitchell, 1997; Seng \& Seng, 1996; Smith \& Tillema, } \\
\text { 1998/2001/2003; Snadden, Thomas, Griffin, \& Hudson, 1996; } \\
\text { Spence \& El-Ansari, 2004; Stewart, 2004; Swallow, Clarke, Iles, \& } \\
\text { Harde, 2006; Tigelaar, Dolmans, De Grave, Wolfhagen, Van der } \\
\text { Vleuten, 2006; Tigelaar et al., 2006; Tillema, 2001; Tisani, } \\
\text { 2008;Tucker, Stronge, Gareis, Beers, 2003; Wildy \& Wallace, 1998. }\end{array}$ \\
\hline $\begin{array}{l}\text { Stimulate reflection/(reflective) } \\
\text { learning (21) }\end{array}$ & $\begin{array}{l}\text { Bunker \& Leggett, 2004; Dixon, Dixon, \& Pelliccione, 2005; Dornan, } \\
\text { Carroll, \& Parboosingh, 2002; Ekbatani \& Pierson, 1997; Guaglia- } \\
\text { none \& Yerkes, 1998; Kjaer, Maagaard, \& Wied, 2006; Lyons, 1998; } \\
\text { Lyons \& Evans, 1997; Marcoux, Brown, Irby \& Lara-Alecio, 2003; } \\
\text { Mathers, Challis, Howe, \& Field, 1999; Orland-Barak, 2005; Pearson } \\
\text { \& Heywood, 2004; Riggs, Sandlin, Scott, Childress, \& Mitchell, 1997; } \\
\text { Seng \& Seng, 1996; Smith \& Tillema, 1998/2003; Snadden \& Tho- } \\
\text { mas, 1998; Spence \& El-Ansari, 2004; Swallow, Clarke, lles, \& Har- } \\
\text { den, 2006; Tigelaar et al., 2006; Webb, Aprahamian, Weigelt, \& } \\
\text { Brasel, 2006. }\end{array}$ \\
\hline $\begin{array}{l}\text { Provide evidence/control/ } \\
\text { accountability (5) }\end{array}$ & $\begin{array}{l}\text { Dixon, Dixon, \& Pelliccione, 2005; Johnston \& Thomas, 2005; Orl- } \\
\text { and- Barak, 2005; Tucker, Stronge, Gareis, Beers, 2003; Wildy \& } \\
\text { Wallace, } 1998 .\end{array}$ \\
\hline $\begin{array}{l}\text { Demonstrating/documenting/ } \\
\text { recording (5) }\end{array}$ & $\begin{array}{l}\text { Bunker \& Leggett, 2004; Dixon, Dixon, \& Pelliccione, 2005; Jun, } \\
\text { Anthony, Achrazoglou, \& Coghill-Behrends, 2007; Lyons \& Evans, } \\
\text { 1997; Tucker, Stronge, Gareis, Beers, } 2003 .\end{array}$ \\
\hline $\begin{array}{l}\text { (re)Certification/Licensure/ } \\
\text { Tenure/promotion/selection/ } \\
\text { award (5) }\end{array}$ & $\begin{array}{l}\text { Bradshaw \& Hawk, 1996; Bunker \& Leggett, 2004; Grandgenette, } \\
\text { 1999; Smith \& Tillema, 2001/2003. }\end{array}$ \\
\hline $\begin{array}{l}\text { Prepare job interview/job appli- } \\
\text { cation/(external) selection or } \\
\text { mobility (4) }\end{array}$ & $\begin{array}{l}\text { Guaglianone, 1995; Brown \& Irby, 1998; Bunker \& Leggett, 2004; } \\
\text { Sullivan, 2004. }\end{array}$ \\
\hline Receive/optimize coaching (1) & Firssova, 2006 \\
\hline Stimulate confidence (1) & Jun, Anthony, Achrazoglou, \& Coghill-Behrends, 2007. \\
\hline To organize oneself (1) & Wildy \& Wallace, 1998 \\
\hline
\end{tabular}

9 Some studies are classified under more than one goal. 
Table 7

Which goals is PDP assessment effective for?

\begin{tabular}{l} 
Effective for... \\
\hline Personal/ (continuing) professional \\
development (17) \\
Stimulating (self-)reflection (reflective \\
learning) (18)
\end{tabular}

Improving the professional practice/performance (5)

Demonstrating/documenting/recording (4)

Stimulating confidence (and selfawareness) of the employee (3) Undertaking more educational/learning activities (2)

Preparing job interviews/job applications (2)

Optimizing coaching (by men-

tor/principal) (3)

Providing constructive feedback (2)

Diagnosing (1)

(re)certification (1)

Facilitating accountability (1)

Facilitating the employee's autonomy

(2)

Self-assessment (1)

Organizing (1)
References

Beck, Livne, \& Bear, 2005; Bullock, Firmstone, Frame, \& Bedward, 2007; Cayne, 1995; Evans, Ali, Singleton, Nolan, \& Bahrami, 2002; Fenwick, 2003; Grandgenette, 1999; Guaglianone \& Yerkes, 1998; Jun, Anthony, Achrazoglou, \& Coghill-Behrends, 2007; Lyons \& Evans, 1997; Marcoux, Brown, Irby, \& Lara-Alecio, 2003; Pitts, Curtis, While, \& Holloway, 1999; Riggs, Sandlin, Scott, Childress, \& Mitchell, 1997; Seng \& Seng, 1996; Stewart, 2004; Swallow, Clarke, lles, \& Harden, 2006; Webb, Aprahamian, Weigelt, \& Brasel, 2006; Wildy \& Wallace, 1998.

Bradshaw \& Hawk, 1996; Cayne, 1995; Dornan, Carroll, \& Parboosingh, 2002; Fenwick, 2003; Joyce, 2005; Kjaer, Maagaard, \& Wied, 2006; Lyons, 1998; Lyons \& Evans, 1997; Marcoux, Brown, Irby, \& Lara-Alecio, 2003; Mathers, Challis, Howe, \& Field, 1999; Pearson \& Heywood, 2004; Pinder \& Turnbull, 2003; Riggs, Sandlin, Scott, Childress, \& Mitchell, 1997; Seng \& Seng, 1996; Snadden, Thomas, Griffin, \& Hudson, 1996; Spence \& El-Ansari, 2004; Tigelaar et al., 2006; Tillema, 2001.

Bahrami, Rogers, \& Singleton, 1995; Evans, Ali, Singleton, Nolan, \& Bahrami, 2002; Joyce, 2005; Marcoux, Brown, Irby, \& Lara-Alecio, 2003; Wildy \& Wallace, 1998.

Jun, Anthony, Achrazoglou, \& Coghill-Behrends, 2007; Lammintakanen, Saranto, Kivinen, \& Kinnunen, 2002; Pinder \& Turnbull, 2003; Tucker, Stronge, Gareis, \& Beers, 2003. Evans, Ali, Singleton, Nolan, \& Bahrami, 2002; Guaglianone \& Yerkes, 1998; Snadden \& Thomas, 1998.

Bahrami, Rogers, \& Singleton, 1995; Bullock, Firmstone, Frame, \& Bedward, 2007.

Guaglianone, 1995; Sullivan, 2004.

Bradshaw \& Hawk, 1996; Firssova, 2006; Kjaer, Maagaard, \& Wied, 2006.

Ekbatani \& Pierson, 1997; Smith \& Tillema, 1998.

Ekbatani \& Pierson, 1997.

Grandgenette, 1999.

Joyce, 2005.

Joyce, 2005; Kjaer, Maagaard, \& Wied, 2006.

Smith \& Tillema, 2001.

Kjaer, Maagaard, \& Wied, 2006. 
Table 8

Supporting process conditions: Object of study.

\begin{tabular}{|c|c|c|}
\hline \multicolumn{2}{|c|}{ Supporting process conditions } & \multirow[b]{2}{*}{$\begin{array}{l}\text { References } \\
\text { Bullock, Firmstone, Frame, \& Bedward, } \\
\text { 2007; Daniel \& Stallion, 1995; Evans et al., } \\
\text { 2002; Fenwick, 2003; Firssova, 2006; Kjaer, } \\
\text { Maagaard, Wied, 2006; Pearson \& Hey- } \\
\text { wood, 2004; Riggs, Sandlin, Scott, Childress, } \\
\text { \& Mitchell, 1997; Snadden, Thomas, Griffin, } \\
\text { \& Hudson, 1996; Tigelaar, Dolmans, De } \\
\text { Grave, Wolfhagen, \& Van der Vleuten, } \\
\text { 2006; Tisani, 2008. }\end{array}$} \\
\hline Support & $\begin{array}{l}\text { From coach (modeling/mentorship/ } \\
\text { supervision) (11) }\end{array}$ & \\
\hline & From col- & Beck et al., 2005; Moore \& Bond, 2002; \\
\hline & leagues(peers)/collaboration (4) & Seng \& Seng, 1996; Tigelaar et al., 2006. \\
\hline & Feedback (4) & $\begin{array}{l}\text { Ekbatani \& Pierson, 1997; Hrisos, Illing, \& } \\
\text { Burford, 2008; Smith \& Tillema, 1998, } \\
\text { Webb, Aprahamian, Weigelt, \& Brasel, } \\
2006 .\end{array}$ \\
\hline & Training lessons for employees (1) & Dornan, Carroll, \& Parboosingh, 2002. \\
\hline & $\begin{array}{l}\text { Clear goal, structure/framework } \\
\text { (also meetings), guidelines, stan- } \\
\text { dards (formalized) (2) }\end{array}$ & $\begin{array}{l}\text { Guaglianone, 1995; Kjaer, Maagaard, Wied, } \\
\text { 2006; }\end{array}$ \\
\hline & $\begin{array}{l}\text { Learning environment (organiza- } \\
\text { tional culture/climate, community } \\
\text { of practice) ( } 2 \text { ) }\end{array}$ & $\begin{array}{l}\text { Johnston \& Thomas, 2005; Daniel \& Stal- } \\
\text { lion, } 1995 .\end{array}$ \\
\hline & Local networks (1) & Bahrami, Rogers, \& Singleton, 1995. \\
\hline & Teaching in courses (1) & Dornan, Carroll, \& Parboosingh, 2002. \\
\hline & $\begin{array}{l}\text { Technical/administrative support } \\
\text { (4) }\end{array}$ & $\begin{array}{l}\text { Kjaer, Maagaard, Wied, 2006; Moore \& } \\
\text { Bond, 2002; Tisani, 2008; Wildy \& Wallace, } \\
\text { 1998. }\end{array}$ \\
\hline \multirow[t]{9}{*}{$\begin{array}{l}\text { Individual cha- } \\
\text { racteristics }\end{array}$} & Confidence/trust (4) & $\begin{array}{l}\text { Fenwick, 2003; Joyce, 2005; Snadden \& } \\
\text { Thomas, 1998; Tisani, } 2008 .\end{array}$ \\
\hline & Learning style (1) & Dornan, Carroll, \& Parboosingh, 2002. \\
\hline & Feasibility ('do-able') (2) & $\begin{array}{l}\text { Hrisos, Illing, \& Burford, 2008; Smith \& } \\
\text { Tillema, } 2003 .\end{array}$ \\
\hline & Motivation (2) & $\begin{array}{l}\text { Bahrami, Rogers, \& Singleton, 1995; Kjaer, } \\
\text { Maagaard, Wied, 2006; }\end{array}$ \\
\hline & Sustained use (2) & $\begin{array}{l}\text { Hrisos, Illing, \& Burford, 2008; Smith \& } \\
\text { Tillema, 1998. }\end{array}$ \\
\hline & Perceived importance (2) & $\begin{array}{l}\text { Tisani, 2008; Webb, Aprahamian, Weigelt, } \\
\text { \& Brasel, } 2006 .\end{array}$ \\
\hline & Age (1) & $\begin{array}{l}\text { Webb, Aprahamian, Weigelt, \& Brasel, } \\
2006 .\end{array}$ \\
\hline & Reflection (1) & Tisani, 2008. \\
\hline & Self-efficacy (1) & Tisani, 2008. \\
\hline \multicolumn{2}{|l|}{$\begin{array}{l}\text { Mandatory/ } \\
\text { voluntary (1) }\end{array}$} & Beck, Livne \& Bear, 2005. \\
\hline Time (7) & & $\begin{array}{l}\text { Bahrami, Rogers, \& Singleton, 1995; Daniel } \\
\text { \& Stallion, 1995; Hrisos, Illing, \& Burford, } \\
\text { 2008; Kjaer, Maagaard, Wied, 2006; Pitts, } \\
\text { Curtis, While, \& Holloway, 1999; Seng \& }\end{array}$ \\
\hline
\end{tabular}




\begin{tabular}{ll}
\hline & Seng, 1996; Tisani, 2008. \\
Resources (3) & Bahrami, Rogers, \& Singleton, 1995; Dor- \\
& nan, Carroll, \& Parboosingh, 2002; Pitts, \\
& Curtis, While, \& Holloway, 1999. \\
\hline
\end{tabular}

Table 9

Supporting process conditions mentioned in literature

\begin{tabular}{|c|c|c|}
\hline \multicolumn{2}{|c|}{ Supporting process conditions } & \multirow[b]{2}{*}{$\begin{array}{l}\text { References } \\
\text { Austin, Marini, \& Desroches, 2005; Beck, } \\
\text { Livne, \& Bear, 2005; Bradshaw \& Hawk, } \\
\text { 1996; Bunker \& Leggett, 2004; Cayne, } \\
\text { 1995; Daniel \& Stallion, 1995; Dornan, } \\
\text { Carroll, \& Parboosingh, 2002; Mathers, } \\
\text { Challis, Howe, \& Field, 1999; Moore \& } \\
\text { Bond, 2002; Pinder \& Turnbull, 2003; } \\
\text { Pitts, Curtis, While, \& Holloway, 1999. }\end{array}$} \\
\hline Support & In general (facilitation)(11) & \\
\hline & \multirow[t]{2}{*}{$\begin{array}{l}\text { From coach (modeling/mentorship/ } \\
\text { supervision) (10) }\end{array}$} & $\begin{array}{l}\text { Bahrami, Rogers, \& Singleton, 1995; } \\
\text { Brashaw \& Hawk, 1996; Joyce, 2005; Little }\end{array}$ \\
\hline & & $\begin{array}{l}\text { \& Hayes, 2003; Lyons, 1998; Marcoux, } \\
\text { Brown, Irby \& Lara-Alecio, 2003; Moore \& } \\
\text { Bond, 2002; Snadden \& Thomas, 1998; } \\
\text { Swallow, Clarke, lles, \& Harden, 2006; } \\
\text { Tigelaar et al., 2006b. }\end{array}$ \\
\hline & From colleagues(peers) (9) & $\begin{array}{l}\text { Austin, Marini, \& Desroches, 2005; Bah- } \\
\text { rami, Rogers, \& Singleton, 1995; Beck, } \\
\text { Livne, \& Bear, 2005; Firssova, 2006; Little } \\
\text { \& Hayes, 2003; Lyons, 1998; Stewart, } \\
\text { 2004; Tigelaar, Dolmans, De Grave, Wolf- } \\
\text { hagen, \& Van der Vleuten, 2006; Tigelaar } \\
\text { et al., 2006b. }\end{array}$ \\
\hline & Feedback (5) & $\begin{array}{l}\text { Pitts, Curtis, While, \& Holloway, 1999; } \\
\text { Tillema, 2001; Tillema \& Smith, 2000; } \\
\text { Smith \& Tillema, 2001/2003. }\end{array}$ \\
\hline & Training lessons for employees (3) & $\begin{array}{l}\text { Austin, Marini, \& Desroches, 2005; Beck, } \\
\text { Livne, \& Bear, 2005; Swallow, Clarke, lles, } \\
\text { \& Harden, 2006. }\end{array}$ \\
\hline & $\begin{array}{l}\text { Clear goal, structure/framework (also } \\
\text { meetings), guidelines, standards } \\
\text { (formalized) (13) }\end{array}$ & $\begin{array}{l}\text { Austin, Marini, \& Desroches, 2005; Beck, } \\
\text { Livne, \& Bear, 2005; Bradshaw \& Hawk, } \\
\text { 1996; Bullock, Firmstone, Frame, \& Bed- } \\
\text { ward, 2007; Cayne, 1995; Daniel \& Stal- } \\
\text { lion, 1995; Dixon, Dixon, \& Pelliccione, } \\
\text { 2005; Pinder \& Turnbull, 2003; Snadden \& } \\
\text { Thomas, 1998; Snadden, Thomas, Griffin, } \\
\text { \& Hudson, 1996; Tillema, 2001; Smith \& } \\
\text { Tillema, 2001/1998. }\end{array}$ \\
\hline & For mentor (2) & $\begin{array}{l}\text { Bradshaw \& Hawk, 1996; Jun, Anthony, } \\
\text { Achrazoglou, \& Coghill-Behrends, } 2007 .\end{array}$ \\
\hline & Collaboration (7) & $\begin{array}{l}\text { Bradshaw \& Hawk, 1996; Daniel \& Stal- } \\
\text { lion, 1995; Moore \& Bond, 2002; Pinder \& }\end{array}$ \\
\hline
\end{tabular}


Turnbull, 2003; Pitts, Curtis, While, \& Holloway, 1999; Riggs, Sandlin, Scott, Childress, \& Mitchell, 1997; Smith \& Tillema, 2001.

Learning environment (organizational culture/climate, community of practice) (7)

Individual characteristics

Reflection (13)
Self-directedness (6)

Confidence/trust (5)

Flexibility (2)

Autonomy (7)

Writing skills (1)

Learning style (1)

Connection with day-to-day practice (see the personal relevance) (9)

Answering the employee's learning needs (1)

Beliefs (2)
Dixon, Dixon, \& Pelliccione, 2005; Dornan, Carroll, \& Parboosingh, 2002; Joyce, 2005; Little \& Hayes, 2003; Pinder \& Turnbull, 2003; Swallow, Clarke, Iles, \& Harden, 2006; Wildy \& Wallace, 1998.

Austin, Marini, \& Desroches, 2005; Beck, Livne, \& Bear, 2005; Bradshaw \& Hawk, 1996; Joyce, 2005; Jun, Anthony, Achrazoglou \& Coghill-Behrends, 2007; Lyons \& Evans, 1997; Mathers, Challis, Howe, \& Field, 1999; Moore \& Bond, 2002; Pinder \& Turnbull, 2003; Seng \& Seng, 1996; Snadden, Thomas, Griffin, \& Hudson, 1996; Swallow, Clarke, lles, \& Harden, 2006; Smith \& Tillema, 1998.

Austin, Marini, \& Desroches, 2005; Joyce, 2005; Little \& Hayes, 2003; Lyons \& Evans, 1997; Pinder \& Turnbull, 2003; Smith \& Tillema, 1998.

Pitts, Curtis, While, \& Holloway, 1999; Stewart, 2004; Smith \& Tillema, 2003; Swallow, Clarke, lles, \& Harden, 2006; Tigelaar, Dolmans, De Grave, Wolfhagen, Van der Vleuten, 2006.

Evans, Ali, Singleton, Nolan, \& Bahrami, 2002; Fenwick, 2003.

Austin, Marini, \& Desroches, 2005; Joyce, 2005; Moore \& Bond, 2002; Orland-Barak, 2005; Tigelaar, Dolmans, De Grave, Wolfhagen, Van der Vleuten, 2006; Tillema, 2006; Smith \& Tillema, 1998; Wildy \& Wallace, 1998.

Cayne, 1995.

Snadden \& Thomas, 1998.

Austin, Marini, \& Desroches, 2005; Bahrami, Rogers, \& Singleton, 1995; Bradshaw \& Hawk, 1996; Bullock, Firmstone, Frame, \& Bedward, 2007; Daniel \& Stallion, 1995; Evans, Ali, Singleton, Nolan, \& Bahrami, 2002; Joyce, 2005; Little \& Hayes, 2003; Snadden, Thomas, Griffin, \& Hudson, 1996.

Bullock, Firmstone, Frame, \& Bedward, 2007.

Pearson \& Heywood, 2004; Tigelaar et al., 2006. 
Mandatory/

voluntary (6)

Time (11)

Resources (4)
Bunker \& Leggett, 2004; Daniel \& Stallion, 1995 (M); Joyce, 2005 (M); Jun, Anthony, Achrazoglou \& Coghill-Behrends, 2007; Swallow, Clarke, lles, \& Harden, 2006 (not M); Smith \& Tillema, 2001 (should be V). Bullock, Firmstone, Frame, \& Bedward, 2007; Bunker \& Leggett, 2004; Firssova, 2006; Joyce, 2005; Little \& Hayes, 2003; Lyons \& Evans, 1997; Pearson \& Heywood, 2004; Riggs, Sandlin, Scott, Childress, \& Mitchell, 1997; Tigelaar, Dolmans, De Grave, Wolfhagen, Van der Vleuten, 2006; Tucker, Stronge, Gareis, \& Beers, 2003; Smith \& Tillema, 1998. Austin, Marini, \& Desroches, 2005; Bradshaw \& Hawk, 1996; Bullock, Firmstone, Frame, \& Bedward, 2007; Daniel \& Stallion, 1995. 


\section{CHAPTER 3}

\section{THE EFFECT OF USING A PERSONAL DEVELOPMENT PLAN (PDP) ON THE PHARMACY ASSISTANT'S LEARNING AND PERFORMANCE ${ }^{10}$}

In order to promote continuing professional development, more and more companies are starting to implement Personal Development Plans (PDPs). Empirical studies researching the effectiveness of PDPs in the workplace are scarce, however. To help rectify this, this study examines the effects of using a PDP on the undertaking of learning activities and the employee's job competencies. To that end data from Dutch pharmacy assistants was collected $(\mathrm{N}=2271)$. ANOVAs as well as regression analyses were conducted on this dataset. The results indicate that the effects of using a PDP in the workplace are not straightforward. PDP users undertook more learning activities in the past than non-users, but using a PDP does not stimulate users to plan more learning activities in the future. Furthermore, PDP users do not score themselves significantly higher on job competencies than non-PDP users. It can be concluded that PDPs are especially used as feedback tools and not as feed forward tools that stimulate individuals to invest in their development.

\section{Introduction}

Since the nineties health services (pharmacists, general practitioners, dentists, consultants, nurses) have come to realize the importance of investing in the development of their professionals and have put continuing professional development (CPD) high on their strategic agendas. The International Pharmaceutical Federation defines CPD as: "the responsibility of individual pharmacists for systematic maintenance, development and broadening of knowledge, skills and attitudes, to ensure continuing competence as a professional, throughout their careers." (International

10 Based upon Beausaert, S., Segers, M., Fouarge, D., \& Gijselaers, W. (submitted). The effect of using a Personal Development Plan (PDP) on the pharmacy assistant's learning and performance. 
Pharmaceutical Federation; in Rouse, 2004, p. 2069). In order to support the CPD of professionals in health service, organizations started to implement Personal Development Plans (PDPs). In the UK for example, the government even suggested to make use of the tool the way forward for CPD (Evans, Ali, Singleton, Nolan, \& Bahrami, 2002). In the Dutch pharmacy business, the PDP has only recently been introduced. In 2004, only $2 \%$ of the pharmacy assistants had a PDP. This percentage had risen to $45 \%$ by 2008 (Fouarge, de Grip, Nelen, \& van Breugel, 2008).

In general a PDP can be described as an assessment tool embedded in a larger assessment cycle of development and performance interviews. It is used to gather and document information about the competencies the employee worked on and is planning to further develop in the near future (Beausaert, Segers, van der Rijt, \& Gijselaers, 2011). Although PDPs are widely used, especially within health services, the empirical evidence on its impact and effects is scarce (e.g. Austin, Marini, \& Desroches, 2005; Evans et al., 2002; Zeichner \& Wray, 2001). Moreover, the results of the empirical studies are inconclusive. Our review study (Beausaert et al., 2011) indicates that most empirical studies show that PDP assessment is effective for learning, personal or professional development and improving professional practice; other studies, however, (e.g. Bunker \& Leggett, 2005) do not support these positive findings. Furthermore, previous studies on PDPs are often based on small sample sizes (e.g. Bullock, Firmstone, Frame, \& Bedward, 2007) and therefore do not lead to generalizable results. Similarly, to date, hardly any studies on the effects of PDPs have been using a control group design and therefore could not draw any valid conclusions on the effectiveness of PDPs.

Our study aims to contribute to the current insights on the effects of PDPs in different ways. First, we focus on two effect measures. Given that undertaking learning activities is highly valued in current human resources management (Kraiger, McLinden, \& Casper, 2004) and PDPs are theorized to be supportive in employees' learning, we investigate whether or a PDP effectively supports and stimulates employees to undertake learning activities. Next, we investigate to what extent PDPs are effective in supporting the development of the general and specific key competencies that are defined by the relevant industry as indicators of the employees' performance. Second, by using a large data set, we aim to measure effects which we eventually will be able to generalize to the total population of pharmacy assistants. Third, we use a quasi-experimental design, comparing users to non-users (control group) of PDPs. To control for sectoral differences in the use of PDP and occupational differences in training and competence development, we focus our study on a homogeneous group of employees from a specific health care service sector. We collected data from employees who work in the Dutch pharmacy sector, more precisely from pharmacy assistants, who are the core workers in this industry. They prepare the medication and have personal contact with the clien- 
tele. All members of the group that we collected our date from are female and share the same vocational education background.

In sum, this empirical study examines the effects of using a PDP on learning and job competencies. This leads to the following research question: "Does using a PDP effectively stimulate the undertaking of learning activities and improve job competencies?" More specifically, this study measures three different effects of using a PDP: the correlation with the employee's learning activities undertaken in the past and the effects on the employee's plans to undertake further training and on his/her competence level. A distinction is made between undertaking learning activities in the past and intended future learning activities since PDPs are designed as feedback tools to reflect on past performance and activities as well as identify training needs of the worker and plan future development accordingly (feed forward) (McMullan et al., 2003).

\section{A Personal Development Plan (PDP)}

In general a PDP can be defined as an assessment tool embedded in a larger assessment cycle of development and performance interviews (also called traditional top-down assessment; Hagan, Konopaske, Bernardin, \& Tyler, 2006), used to gather and document information about the competencies the employee has already worked on and the ones that $s /$ he is planning to further develop (Beausaert et al., 2011). More concretely, a PDP can be characterized as a tool that (Brown, 1995; McMullan et al., 2003; Redman, 1994; Seng \& Seng, 1996; Smith \& Tillema, 1998; Snadden, Thomas, Griffin, \& Hudson, 1996):

Gives an overview of the competencies the employee worked on in the past (looking back) and of the competencies the employee is planning to work on in the future (looking forward);

- Is composed by the employee himself (self-direction by the employee), although the format of the PDP is mostly fixed;

- Is used as basis for or to structure the conversations with the supervisor or the coach, who provides the employee with feedback and stimulates the employee's reflection;

- $\quad$ Serves different decision-making processes, ranging from planning an individual training-program to whether or not giving an employee a promotion.

Using a PDP requires the user to act as a reflective learner (Schön, 1987). Reflection signifies a critical analysis of previous experiences and aims at intensifying cognitive elaboration on those experiences; it is expected to lead to behavioral changes (Anseel, Lievens, \& Schollaert, 2009; McMullan et al., 2003; Seng \& Seng, 1996; Smith \& Tillema, 1998). In other words, reflecting provides insight into the employee's 
own processes of learning (McMullan et al., 2003). Moreover, Schön makes a distinction between two ways of engaging in reflection: reflection-in-action and reflection-on-action. Reflection-in-action refers to reflecting during the experience, without interrupting it. It allows the reflective practitioner to adjust what $s /$ he is doing while s/he is doing it. To reflect on action means that we think back on what we have done, after the initial experience (Schön, 1987). This reflection on action can be supported by making use of a PDP. Similarly, Kolb states in his model of experiential learning that learning occurs through 'concrete experiences' and the reflection on those experiences (Kolb, 1984), for which a PDP can be used. By thinking over what happened, the learner is able to draw a more general conclusion (abstraction) and build up concepts (conceptualization). Finally, the learner can use previous experiences and what he learned from those experiences as a basis for new active experimentation, and, finally, an improved performance.

In the PDP, three major feedback questions take up a central role: 'Where am I now?', 'Where am I going?', and 'Where to go next?' The third has a feed-forward function. When a discrepancy is detected between the competencies an employee possesses and the competencies $s /$ he should possess, further learning can be stimulated (Hattie \& Timperley, 2007).

Gathering and documenting information by means of a PDP is used to serve two main purposes. Commonly a distinction is made between professional development and certification/selection/accountability (Smith \& Tillema, 2001). When a PDP is used to support reflection and development, the feedback is mainly used to scaffold learning. Conversely, when a PDP is used for certification, presenting oneself in a positive light is more important (Beck, Livne, \& Bear, 2005; Bradshaw \& Hawk, 1996; Bunker \& Leggett, 2004; Lyons \& Evans, 1997; Orland-Barak, 2005; Smith \& Tillema, 2001). Although there is a trend towards using the tool for certification and performance appraisal, the tool is especially powerful to support employees' professional development (Smith \& Tillema, 2003).

Besides differentiating the aforementioned purposes of a PDP, Smith and Tillema (2001) make a second distinction, between composing a PDP on a voluntary basis and on a mandatory basis. The authors reported that there was more professional development when sustained PDP use was voluntary than when it was mandatory (Smith \& Tillema, 2001). Other research, on the other hand, showed that staff members did not develop a PDP unless this was mandatory (Bunker \& Leggett, 2004).

\section{The effectiveness of PDPs: Previous research and hypotheses}

PDPs are used to steer employees' competence development (see definition). Employees should undertake learning activities and improve their competencies exact- 
ly as a result of using the PDP as a reflection tool. A recent review of 54 studies on the effectiveness of PDP assessment in organizations showed that different studies led to different, often contradicting findings (Beausaert et al., 2011). While most researchers found that in the health care services PDPs facilitate learning activities, help develop professional knowledge and skills, promote reflection and improve performance, others demonstrated that it is difficult for PDPs to stimulate reflection and influence professional practices at the workplace (Beausaert et al., 2011).

Evans et al. (2002), for example, analyzed general practitioners' PDP use by means of a questionnaire, analyses of PDP reports and in-depth interviews. The majority of general practitioners (GPs) felt that the PDP helped them to enhance their personal and professional development effectively as well as to improve their service to patients. Similarly, Mathers and colleagues (1999) concluded that through using PDPs, GPs become more proactive in their learning. In their study, 32 volunteer GPs were divided into two groups. Each group followed a 'traditional' route to postgraduate educational accreditation (PGEA) in the first six months, and then a PDP-based learning route in the next six months. It was found that making a PDP provided proof of the application of learning into professional practice and facilitated the completion of learning cycles. Other researchers measured whether the use of an electronic PDP is effective for reflective CPD of physicians (Dornan, Carroll, \& Parboosingh, 2002). They surveyed physicians who registered with the Royal College of Physicians to work on their CPD. They concluded that although the use of electronic PDPs is influenced and sometimes hampered by individual learning styles, resources and technical support, it does indeed lead to reflective learning.

In contrast, other research suggests that implementing a PDP system in a profession can be quite complex. Because of time pressure, practitioners' self-directed learning inclination and self-monitoring abilities cannot be brought into full play. Although the PDP is consistent with the idea of continuous professional development theoretically, there seems to be a lack of impact from PDPs on professional practice. One study found that pharmacists did not closely and successfully connect documentation and self-reflection with practice improvement (Austin et al., 2005). This is in line with the research of Kostrzewski, Dhillon, Goodsman and Taylor (2009) who undertook an in-depth research to examine the effect of PDPs on the pharmacy practice in hospitals by conducting semi-structured face-to-face interviews. The participants were four male and five female pharmacists, and were divided into three groups. Three key themes kept returning in the participants' answers: lack of contribution to practice, tacit contribution and mentality. Furthermore, it was found that having a PDP has little if any influence on the professional practice, especially for the more experienced participants. The study consequently concluded that it is hard to demonstrate positive effects of the use of PDPs in practice. 
Similarly, Bunker and Leggett (2005; in Leggett \& Bunker, 2006) argued that in the context of higher education teaching PDPs can be effective tools for stimulating promotion, but that they are not useful to encourage reflective practice, because the PDP focuses on success more than on how to avoid failure. Orland-Barak (2005) also doubted the effectiveness of portfolios for reflection. For her research she analyzed 32 teachers' portfolios in two in-service courses for mentors of teachers in Israel. In both product $(n=20)$ and process portfolios $(n=12)$ the documentation of critical reflection was problematic.

Based on these insights, still indicating more positive than negative effects of PDP use (Beausaert et al., 2011), the following hypotheses can be formulated:

- Hypothesis 1 (H1). In contrast to pharmacy assistants who don't use a PDP, pharmacy assistants who use a PDP undertook more learning activities in the past.

- Hypothesis 2 (H2). In contrast to pharmacy assistants who don't use a PDP, pharmacy assistants who do are planning to undertake more learning activities in the future.

- Hypothesis 3 (H3). In contrast to pharmacy assistants who don't use a PDP, pharmacy assistants who do will score themselves higher on job competencies.

\section{Data and method}

\subsection{Participants}

We use data from a large scale survey conducted among Dutch pharmacy employees in 2008. We focus on the pharmacy assistants - the core workers - who account for $80 \%$ of total employment in the pharmacy sector (De Grip \& Sieben, 2009). The advantage of focusing on the core workers is that they are key employees in the branch, and that it is a homogeneous group in terms of educational background and profession.

In March 2008, 6000 pharmacy assistants were asked to take part in the survey. Pharmacy assistants were sampled from the administrative database of the Dutch pension fund for pharmacy workers (PMA), where all pharmacy assistants are required to register. The participants were invited by means of an e-mail which contained a link to the online version of the questionnaire. 2156 pharmacy assistants in total were contacted this way. Because their e-mail address was unknown a further 3844 pharmacy assistants were invited by regular mail which contained a printed version of the questionnaire and a link to the online version. 
2271 females answered the full questionnaire (response rate $38 \%$ ). ${ }^{11}$ The sample is representative for all pharmacy assistants in the branch with respect to their background characteristics. They are, however, on average somewhat older and the data is weighted accordingly. Of the 2247 pharmacy assistants who provided us with their highest level of education, the largest group, 2025 had a degree from secondary vocational education, 141 had a degree from higher vocational education or higher, and 81 had another certificate. The average age of the pharmacy assistants was 39.54 years (SD $=10.17)$. The average number of years of working experience was 19.35 years $(S D=8.80)$. On average an employee had been working as a pharmacy assistant for 16.70 years $(S D=9.26)$, worked or had been working part-time for 11.61 years $(S D=8.62)$ and works 25.27 hours per week $(S D=7.62)$. 1335 had children who are still living at home.

\subsection{Measures}

This study focuses on the effects of using a PDP on three dependent variables: having undertaken learning activities in the past, planning further training, and perceived job competencies. In addition, the analyses control for a number of the assistants' background characteristics: age, total working experience, working experience as a pharmacy assistant, children living at home, number of working hours/week, number of years working part-time.

PDP use. Pharmacy assistants were asked if they make use of a PDP or not. The question "Do you have a PDP?" could be answered by yes (1) or no (0) and consequently indicated two groups, the users and the non-users.

Learning activities. We measured the undertaking of learning activities in the past and in the future. The undertaking of learning activities in the past was measured with a yes/no-question: "Did you undertake one or more learning activities in the past year?". Next, a list was given of 19 competencies relevant for pharmacy assistants and defined as key competences by the sector. The list included general competencies (e.g. communication skills, problem solving, leadership...) as well as occupation-specific skills (e.g. preparation of medicines, knowledge of regulations...). The assistants were asked to indicate the competencies which they are planning to work on by undertaking training courses in the future. A sum score was calculated by counting the number of competencies the pharmacy assistants indicated she wanted to train.

Job competencies. To measure job competencies, a list was given of 19 relevant competencies for pharmacy assistants as defined by the business. They included

11 Only a few pharmacy assistants are male. 
general competencies (e.g. communication skills, problem solving, leadership...) as well as occupation-specific skills (e.g. preparation of medicines, knowledge of regulations...). The participants had to score themselves on each competency on a scale from 1 to 10. A single measure was calculated, based on the average of the respondents' answers.

\subsection{Data analysis}

Firstly, descriptive statistics (means and standard deviations) are compared for users and non-users of a PDP. Secondly, ANOVAs were performed in order to detect significant differences between the two distinct groups concerning the undertaking of learning activities (past and future) and the scoring of their job competencies.

Preliminary analyses of the data involved inspection of normality and homogeneity of variance assumptions. Normal plots, box-plots and the calculation of skewness and kurtosis were used to check the normality of distribution. In order to test the equality of group variances the Levene's statistics were calculated. To distinguish between "practically" significant results and results being "statistically" significant, the effect sizes are reported and the results of the statistical analyses were named significant by a $p$ value of $<.05$.

\section{Results}

Table 1

Descriptive statistics and ANOVAs for users and non-users of a PDP

\begin{tabular}{lccccccc}
\hline \multicolumn{7}{l}{ Variable } & \multicolumn{7}{c}{ PDP users (N=861) } \\
\hline & $\mathrm{N}$ & $\mathrm{M}$ & $\mathrm{SD}$ & $\mathrm{N}$ & $\mathrm{M}$ & $\mathrm{SD}$ & $\mathrm{F}$ \\
\hline $\begin{array}{l}\text { Undertaking learn- } \\
\text { ing activities (past) }\end{array}$ & 1034 & .80 & .40 & 1226 & .92 & .28 & $68.07^{* * *}$ \\
$\begin{array}{l}\text { Undertaking learn- } \\
\text { ing activities (fu- }\end{array}$ & 1040 & 3.35 & 2.06 & 1231 & 3.11 & 1.81 & $8.67^{* *}$ \\
$\begin{array}{l}\text { ture) } \\
\text { Job competencies }\end{array}$ & 1040 & 7.46 & .60 & 1231 & 7.51 & .56 & 3.20 \\
\hline${ }^{*} p<.05 .{ }^{* *} p<.01 .{ }^{* * *} p<.001$. & & & & & & &
\end{tabular}

Table 1 illustrates the descriptives (means and standard deviations) and analysis of variance (ANOVA) for the three outcome variables involved in this study. The results indicate significant differences between the two conditions (PDP use; no PDP use) concerning the undertaking of learning activities in the past $(F(1,2258)=$ $68.07, p<.001)$ and the future $(F(1,2269)=8.67, p<.01)$. In particular, we find that the mean for the undertaking of learning activities in the past is higher for em- 
ployees who use a PDP. In other words, employees who use a PDP undertook significantly more learning activities in the past. This is in line with hypothesis 1 . In contrast, the mean for the undertaking of learning activities in the future is significantly higher for non-users. It seems that PDP users are planning to undertake significant less learning activities in the future than non-PDP users; therefore, we reject hypothesis 2 .

Next, we find that the mean score for the outcome variable job competencies is slightly higher for pharmacy assistants who use a PDP than for employees who do not. The difference between the mean scores of the two groups of employees is not significant $(F(1,2269)=3,20, n s)$, however. This means that hypothesis 3 has to be rejected.

Table 2

Summary of logistic regression analysis for PDP use predicting Undertaking learning activities (past), controlling for background variables (age, total working experience, working experience as pharmacy assistant, children living at home, number of working hours/week, number of years working part-time)

\begin{tabular}{|c|c|c|c|}
\hline \multirow[b]{2}{*}{ Predictor } & \multicolumn{3}{|c|}{$\begin{array}{l}\text { Undertaking learning activities } \\
\text { in the past }\end{array}$} \\
\hline & $B$ & $S E B$ & $e^{B}$ \\
\hline \multicolumn{4}{|l|}{ Background variables } \\
\hline Age & -.01 & .01 & .99 \\
\hline Working experience (total) & .02 & .02 & 1.0 \\
\hline Working experience as pharmacy assistant & .01 & .01 & 1.01 \\
\hline Number of years working part-time & .00 & .01 & 1.00 \\
\hline Number of working hours/week & $.02 *$ & .01 & 1.02 \\
\hline Children living at home & .17 & .15 & 1.19 \\
\hline PDP use & $1.02 * * *$ & .13 & 2.77 \\
\hline Constant & .65 & .48 & 1.91 \\
\hline$\chi^{2}$ & $75.22 * * *$ & & \\
\hline$D f$ & 7 & & \\
\hline $\mathrm{N}$ & 861 & & \\
\hline
\end{tabular}


Table 3

Summary of hierarchical regression analysis for PDP use predicting Planning future learning activities, controlling for background variables (age, total working experience, working experience as pharmacy assistant, children living at home, number of working hours/week, number of years working part-time)

\begin{tabular}{|c|c|c|}
\hline & \multicolumn{2}{|c|}{$\begin{array}{l}\text { Planning future lear- } \\
\text { ning activities }\end{array}$} \\
\hline & $\beta$ & $\Delta R^{2}$ \\
\hline \multicolumn{3}{|l|}{ Step 1} \\
\hline Age & .04 & \\
\hline Working experience (total) & .08 & \\
\hline Working experience as pharmacy assistant & $-.15^{* * *}$ & \\
\hline Number of years working part-time & -.05 & \\
\hline Number of working hours/week & .03 & \\
\hline \multirow[t]{2}{*}{ Children living at home } & -.04 & \\
\hline & & $.02 * * *$ \\
\hline \multicolumn{3}{|l|}{ Step 2} \\
\hline Age & .04 & \\
\hline Working experience (total) & .08 & \\
\hline Working experience as pharmacy assistant & $-.15^{* * *}$ & \\
\hline Number of years working part-time & -.05 & \\
\hline Number of working hours/week & .03 & \\
\hline Children living at home & -.04 & \\
\hline \multirow[t]{2}{*}{ PDP use } & $-.07 * *$ & \\
\hline & & $.00 * *$ \\
\hline
\end{tabular}

Note. The reported regression coefficients are unstandardized (B) and unstandardized $(\beta)$ coefficients. ${ }^{*} p<.05 .{ }^{* *} p<.01 .{ }^{* * *} p<.001$.

Follow-up regression analyses with PDP use as independent variables and both significant outcome variables as dependent variables, controlling for background variables, resulted in the same findings. Although the Nagelkerke $R^{2}$ was low, but significant $\left(R^{2}=.02\right)$, the results indicate that PDP users report having undertaken more learning activities in the past (Table 2 ), however, they seem to plan less learning activities in the future (Table 3). Furthermore, a significant relation was found between number of working hours per week and the undertaking of learning activities in the past. The more hours a pharmacy assistant works per week, the bigger the chance she undertook learning activities in the past $(\theta=.02, p<.05)$. Next, a significant relation was found between working experience as pharmacy assistant and planning future learning activities. The more experience a pharmacy assistant has, the fewer future trainings are planned $(b=-.15, p<.001)$. 


\section{Conclusion and discussion}

As in most other service sectors, PDPs have become increasingly common in the Dutch pharmacy sector as a means to support employees' development. This study analyzed whether Dutch pharmacy assistants' use of PDPs influences the undertaking of learning activities and their job competencies.

The Dutch pharmacy sector is a highly interesting sector for studying the relation between PDP use and the undertaking of learning activities and the development of job competencies, because HRD practices are central to the human resource practices in this sector (De Grip \& Sieben 2009). Moreover, the sector expects increasing shortages of pharmacy assistants within the next few years. This is why the sector needs to invest in the learning and development of the senior pharmacy assistants, to keep them up-to-date. It is of great interest to investigate whether using employee development tools such as personal development plans enhances employees' learning and job competencies.

On the basis of the literature on PDP assessment in organizations we predicted that users of a PDP would generally have undertaken more learning activities in the past as well as undertake more in the future. Our results confirm that users of a PDP undertook more learning activities in the past than non-users. In contrast to our expectations, however, pharmacy assistants who use a PDP do not plan more learning activities in the future, which is to say they do not plan to undertake further training more often than non PDP users. This finding could indicate that PDPs are especially used as feedback tools rather than feed forward tools. In other words, we expect that the tools are often used to discuss learning activities that have already been undertaken, but do not serve as a tool to support employees in the planning of future learning activities. Furthermore, we found that non-users generally plan to undertake significantly more learning activities than PDP-users, which does not support our hypothesis.

With respect to the extent to which pharmacy assistants are competent in their jobs, we have found no significant differences between users and non-users of a PDP. This lack of differences might be explained by the fact that by using a PDP, the pharmacy assistants are more aware of the competencies that are needed to fulfil their jobs because the PDP stimulates them to think about them. They are stimulated to think about the job competencies they already possess and the competencies they still need to develop. This is the core goal of using a PDP: stimulating the employee to think about her/his job profile and the competencies which are needed to fulfil the job and compare them with the competencies s/he already possesses in order to single out those that the employee still needs to develop (Topping, 1998). This reflection process is a crucial element of the learning and development process of the employee, but might also lead to a more negative evaluation by PDP users (compared to non-users) on the extent to which they pos- 
sess the necessary job competencies. This might explain the lack of differences in reported job competencies between both groups.

A second explanation is the influence of the lack of reflection skills. Previous research evidenced that reflection is still a skill that PDP users are unfamiliar with (Smith \& Tillema, 1998); therefore, it is possible that PDP users did not show a significant improvement in job competencies because some of them had difficulties reflecting and further connecting self-reflection and practice improvement (Austin et al., 2005; Orland-Barak, 2005). Not only the reflection plays a central role when using a PDP, also the presence of a supervisor who gives feedback and instructions and motivates the employee to work and reflect on his/her PDP is crucial to the PDP practice. The lack of a supervisor who fulfils this supporting role may also explain not finding the positive effects as hypothesized (London, 1997).

Third, it might be questioned to what extent the lack of effects of PDP use on learning and job competencies is context-specific. Results differ according to the organizational learning culture (Marsick \& Watkins, 2003), sectors or disciplines (e.g. Datta, Guthrie, \& Wright, 2005), type of organizations (market-oriented or not; Baker \& Sinkula, 1999), type of employees (e.g. experienced versus nonexperienced) and organization sizes (small versus large-scale; Saru, 2007). Often larger organizations show a more sophisticated human resource management (Guthrie, 2001).

\subsection{Implications for future research}

This quantitative research has some limitations that need to be taken into account. Based on these limitations, we will now make some suggestions for future research.

First, industry-specific studies such as ours have a great advantage, in that they can focus on a rather homogeneous workforce. However, for crossvalidation purposes our results need to be reproduced in other industries. Future research should investigate the effectiveness of PDPs for the undertaking of learning activities and job competencies in a broader sample which covers professions that differ in labour market perspective (e.g. in terms of current and future shortage of labour force) or in terms of job demand and job control. Moreover, since our data set consisted of female workers only, it would be interesting to reproduce our analysis in industry sectors with a mixed gender population.

Second, this study was a cross-sectional research. However, it has been demonstrated that high quality of a PDP can only be expected after a longer period of time (Smith \& Tillema, 1998). Thus, a similar study within a longitudinal design could more accurately measure the effectiveness of PDP in the workplace.

Third, future research should explore opportunities that not only use selfreport measures, but involve multiple raters as well. This is especially true when 
measuring effects of an intervention such as a PDP on employees' behaviour in terms of undertaking learning activities and improving job competencies. Using self-report measures, however, remains a powerful method for the measurement of the influence of supporting conditions.

Fourth, the predictive value $\left(R^{2}\right)$ is significant, however, rather low. For future research we suggest to focus more closely on one essential feature of the PDP practice: the feedback given by a supervisor and/or colleague or coach when discussing the PDP. The quality of that feedback might influence the use of a PDP to a large extent. The feedback, for example, often focuses on the individual's job performance in terms of how s/he performed the daily tasks and is not explicitly related to the employee's competencies (Hattie \& Timperley, 2007; Smith \& Tillema, 2001). This might explain why in our study there is no measurable effect of the use of a PDP on job competencies. Furthermore, feedback often tends to be retrospective and does not include a prospective aspect (feed forward), discussing the question 'which steps need to be taken next?'. Future research could verify the effect of this 'feed forward' on the undertaking of learning activities and on job performance.

Fifth, it is possible that comparing users and non-users resulted in biased findings. For example, it could be that a PDP is used especially by employees who are very interested in developing and really motivated to learn. Research using randomized samples might solve this problem.

Sixth, the results indicate a very small amount of variance that is accounted for by the variables studied. Future research should also examine other dependent variables, and consequently might shed a light on the practical significance of the research findings.

\subsection{Implications for practice}

This research yields some implications for human resource management in organizations and the supervisors who support employees in using a PDP. The results stress the value of a PDP as a feed-back tool. The tool could add significant value to the learning and development process of the pharmacy assistant, however, if it would be used as a feed-forward instrument as well. In other words, the tool should more often be used to get an overview of desired future plans, plan future careers, and the undertaking of learning activities in order to reach these future goals. In line with the adult learning theories (Schön, 1987; Kolb, 1984; Boud, 1985) it can be stated that only when supervisors stimulate their employees to use the PDP as a tool to look forward, reflection upon action as well as acting upon their reflection is stimulated and it may be expected that employees' job performance quality will improve. 
Based on the results of this study, we suggest setting up programs to introduce supervisors in the use of PDPs. It is only when the supervisors are giving feedback (looking back) as well as feedforward (looking forward) and thereby support their employees with planning learning activities in the future, we expect the PDP practice to really encourage employees' competence development. 


\section{References}

Anseel, F., Lievens, F., \& Schollaert, E. (2009). Reflection as a strategy to enhance task performance after feedback. Organizational Behavior and Human Decision Processes, 110, 23-35.

Austin, Z., Marini, A., \& Desroches, B. (2005). Use of a learning portfolio for continuous professional development: A study of pharmacists in Ontario (Canada). Pharmacy Education, 5, 175-181.

Baker, W.E., \& Sinkula, J.M. (1999). The synergistic effect of market orientation and learning orientation on organizational performance. Journal of the Academy of Marketing Science, 27, 411-427.

Beausaert, S., Segers, M., van der Rijt, J., Gijselaers, W. (2011). The use of Personal Development Plans in the workplace: A literature review. In P. van den Bossche, W. Gijselaers, R. Milter (Eds.), Advances in Business Education and Training III (pp. 235-265). Dordrecht: Springer.

Beck, R.J., Livne, N.L., \& Bear, S.L. (2005). Teachers' self-assessment of the effects of formative and summative electronic portfolios on professional development. European Journal of Teacher Education, 28, 221-244.

Boud, D., Keogh, R., \& Walker, D. (Eds.) (1985). Reflection: Turning Experience into Learning. London: Kogan Page.

Bradshaw, L.K., \& Hawk, P.P. (1996). Portfolios for the continuing licensure of beginning teachers: Their development and assessment. Paper presented at the Annual Meeting of the National Staff Development Council (Vancouver, British Columbia, Canada, December 10, 1996).

Brown, R. (1995). Portfolio development and profiling for nurses, $2^{\text {nd }}$ edn. Lancaster: Quay Publications.

Bullock, A., Firmstone, V., Frame, J., \& Bedward, J. (2007). Enhancing the benefit of continuing professional development: A randomized controlled study of personal development plans for dentists. Learning in Health and Social Care, 6 (1), 14-26.

Bunker, A., \& Leggett, M. (2004). Being wise about teaching portfolios: Exploring the barriers to their development and maintenance. Retrieved from http://www.herdsa.org.au/wpcontent/uploads/ conference/2004/PDF/P047-jt.pdf on 20/09/2007.

Datta, D.K.,Guthrie, J.P., \& Wright, P.M. (2005). Human resource management and labor productivity: Does industry matter? Academy of Management Journal, 48, 135-145.

De Grip, A., \& Sieben, I. (2009). The Effectiveness of More Advanced Human Resource Systems in Small Firms. International Journal of Human Resource Management, 20, 1914-1928.

Dornan, T., Carroll, C., \& Parboosingh, J. (2002). An electronic learning portfolio for reflective continuing professional development. Medical Education, 36, 767-769.

Evans, A., Ali, S., Singleton, C., Nolan, P., \& Bahrami, J. (2002). The effectiveness of personal education plans in CPD: An evaluation. Medical Teacher, 24 (1), 79-84.

Fouarge, D., de Grip, A., Nelen, A., \& van Breugel, G. (2008). Arbeidsmarktmonitor Apotheekbranche 2007-2008. Nieuwegein: SBA [Labour market Monitor for the pharmacy sector 2007-2008].

Guthrie, J.P. (2001). High-Involvement Work Practices, Turnover, and Productivity: Evidence from New Zealand. The Academy of Management Journal, 44, 180-190.

Hagan, C.M., Konopaske, R., Bernardin, H.J., \& Tyler, C.L. (2006). Predicting assessment center performance with 360-degree, top-down, and customer-based competency assessment. Human Resource Management, 45, 357-390.

Hattie, J., \& Timperley, H. (2007). The power of feedback. Review of Educational Research, 77, 81-112.

Kolb, D.A. (1984). Experiential Learning. Chicago: Prentice Hall.

Kostrzewski, A. J., Dhillon, S., Goodsman, D., \& Taylor, K. M. G. (2009). The influence of continuing professional development portfolio records on pharmacy practice. The International Journal of Pharmacy Practice, 17 (2), 107-113.

Kraiger, K., McLinden, D., Casper, W.J. (2004). Collaborative planning for training impact. Human Resource Management, 43, 337-351.

Leggett, M., \& Bunker, A. (2006). Teaching portfolios and university culture. Journal of Further and Higher Education, 30, 269-282. 
London, M. (1997). Job feedback: Giving, seeking, and using feedback for performance improvement. Mahwah, NJ: Erlbaum.

Lyons, N., \& Evans, L. (1997). Portfolio: A tool for self-directed learning at work. Paper presented at the Self-directed Learning: Past and Future Symposium (Montreal, Quebec, Canada, September 1997).

Marsick, V.J. \& Watkins, K.E. (2003). Demonstrating the value of an organization's learning culture: The dimensions of the learning organization questionnaire. Advances in Developing Human Resources, 5, 132-151.

Mathers, N.J., Challis, M.C., Howe, A.C., \& Field, N.J. (1999). Portfolios in continuing medical education effective and efficient? Medical Education, 33, 521-530.

McMullan, M., Endacott, R., Gray, M.A., Jasper, M., Miller, C.M.L., Scholes, J., \& Webb, C. (2003). Portfolios and assessment of competence: A review of the literature. Journal of Advanced Nursing, 41, 283-294.

Orland-Barak, L. (2005). Portfolios as evidence of reflective practice: What remains "untold". Educational Research, 47 (1), 25-44.

Redman, W. (1994). Portfolios for development: A guide for trainers and managers. London: Kogan Page.

Rouse, M. (2004). Continuing professional development in pharmacy. American Journal of Health System Pharmacy, 61 (19), 2069.

Saru, E. (2007). Organisational learning and HRD: how appropriate are they for small firms? Journal of European Industrial Training, 31 (1), $36-51$.

Schön, D. A. (1987). Educating the Reflective Practitioner. San Francisco: Oxford.

Seng, S.H., \& Seng, T.O. (1996). Reflective teaching and the portfolio approach in early childhood staff development. Paper presented at the Joint Conference of the Educational Research Association of Singapore and the Australian Association for Research in Education. Singapore, 25-29 November 1996.

Smith, K., \& Tillema, H. (1998). Evaluating portfolio use as a learning tool for professionals. Scandinavian Journal of Educational Research, 42, 193-205.

Smith, K., \& Tillema, H. (2001). Long-term influences of portfolios on professional development. Scandinavian Journal of Educational Research, 45, 183-202.

Smith, K., \& Tillema, H. (2003). Clarifying different types of portfolio use. Assessment \& Evaluation in Higher Education, 28, 625-648.

Snadden, D., Thomas M.L., Griffin, E.M., \& Hudson, H. (1996). Portfolio-based learning and general practice vocational training. Medical Education, 30, 148-152.

Topping, K. (1998). Peer assessment between students in colleges and universities. Review of Educational Research, 68 (3), 249.

Zeichner, K., \& Wray, S. (2001). The teaching portfolio in US teacher education programs: what we know and what we need to know. Teaching and Teacher Education, 17, 613-621. 


\section{CHAPTER 4}

\section{USING A PDP FOR DIFFERENT PURPOSES: ITS INFLUENCE ON UNDERTAKING LEARNING ACTIVITIES AND JOB PERFORMANCE ${ }^{12}$}

Today, organizations are increasingly implementing assessment tools such as Personal Development Plans. Although the true power of the tool lies in supporting the employee's continuing professional development, organizations implement the tool for various different purposes, professional development purposes on the one hand and promotion/salary raise/selection/accountability purposes on the other (Smith \& Tillema, 2001). The study presented here aims at a better understanding of how the purpose of the Personal Development Plan (PDP), as perceived by the employee, influences the extent to which $s /$ he undertakes learning activities and consequently leads to improved performance. Data were collected from 286 employees working in a regional Dutch governmental office and 81 experts from an international organization that is specialized in medical technology $(N=367)$. Data were analyzed by conducting hierarchical regression analyses. Results indicate that perceiving the PDP either as a learning and development tool or as a promotion and selection tool, positively predicts the undertaking of learning activities and the employee's performance. Follow-up regression analysis indicated that the most powerful predictor of undertaking learning activities and a high-quality performance is the extent to which employees perceive PDPs as serving learning and development purposes. The results of this study suggest that if an organization wants their employees to learn by undertaking learning activities and in turn perform better, the tool should in the first place be introduced and used as a learning and development tool.

12 Based upon Beausaert, S., Segers, M., \& Gijselaers, W. (accepted). Using a Personal Development Plan for different purposes: Its influence on undertaking learning activities and job performance. Vocations and Learning. 


\section{Introduction}

In recent years organizations have been facing and dealing with fundamental changes that have put the issue of supporting employees' learning and development high on the agenda. Examples of changes are the prevalence of a growing number of short-term contracts, shortage of skilled workers in some industries, ageing workforce and increasing employee mobility. As McDowall and Fletcher formulate (2004, p. 8): “It is in an organization's best interest to focus on effective staff development strategies, since companies strong on training and development may be preferred over those offering the greatest rewards. One technique for developing individuals is through the social and motivational aspects of appraisal or review processes." They define appraisal processes in relation to the formal rating of performance, whereas the term review is used to refer to human resource practices with the purpose of supporting employee's professional growth or development. Popular techniques for enhancing professional development are multisource or 360-degree feedback techniques, career discussions with managers and Personal Development Plans (PDPs). However, as McDowall and Flatcher (2004) argue, most studies to date have tended to concentrate on performance or rewards rather than development, perhaps reflecting the traditional practice of retrospective assessment (Nathan, Mohrmann, \& Milliman, 1991).

In this study, we focus on Personal Development Plans, a strategic developmental tool used by Human Resource Departments to stimulate employees' formal (e.g. training) and informal (e.g. reading a book, peer feedback discussions) learning which in turn is assumed to improve performance. In general a PDP can be described as an assessment tool embedded in a larger assessment cycle of development and appraisal interviews; used to gather and document information about the competencies the employee worked on and is planning to further develop. The PDP is used in hopes that employees would intentionally undertake learning activities and in turn improve workplace performance (London, 1997; van de Wiel, Szegedi, \& Weggeman, 2004).

PDPs have come into widespread use, not only in the fields of medicine and education, but also in business contexts and governmental offices (Beausaert, Segers, van der Rijt, \& Gijselaers, 2011). For example, a survey in the Netherlands among companies in the region of Limburg indicated that in the context of talent management $89 \%$ of the companies are using PDPs (GITP, 2008). In the UK, different authors stress the strong recommendation by the government to use PDPs in order to stimulate the continuous professional development of health service (Evans, Ali, Singleton, Nolan, \& Bahrami, 2002; Bullock, Firmstone, Frame, \& Bedward, 2007).

Despite its popularity, empirical evidence on the effectiveness of PDP's is still scare and mainly limited to educational and health care settings. Moreover, there 
are large variations between the different studies with respect to the features of the implementation of the PDP's. One important difference is the purpose for which PDP are used. A distinction has commonly been made between two broad clusters of purposes, learning/development on the one hand and promotion/selection/accountability on the other (Smith \& Tillema, 2001).

Although different authors question whether PDPs can be discussed in development interviews as well as in appraisal interviews, conducted by one and the same supervisor (e.g. Beck, Livne \& Bear, 2005; Wolf \& Dietz, 1998), hardly any research studies address this issue. Nevertheless, research in related fields offers arguments for considering the purpose for which a PDP is used.

First, when PDP's are used for rewarding purposes (such as promotion or salary increase) instead of developmental purposes, it can be assumed that the openness toward critical self-reflection is jeopardized. However, this critical reflection is the starting point of professional development as it makes clear the strengths and weaknesses in past performance (e.g. Riley-Doucet \& Wilson, 1997). Employees' self-protection and fear of underachieving may lead to the collection of unauthentic evidence and the construction of invalid PDPs, instead of PDPs that openly reflect on the employee's learning and development (Smith \& Tillema, 1998, 2001). As stated by Smith and Tillema (2003: p. 626): "The relation between selection of evidence and reflection on work remains intrinsically tense".

Second, when the purpose of PDPs is not transparent for the employee, implying it is not clear which decisions will be taken based upon the PDP, this might lead to a lack of trust or confidence in the review by the supervisor and in turn hinder professional development and improvement of performance. Research on 360degree feedback has presented evidence on the key role of trust in the assessor. The tool of 360-degree feedback "rests on the assumption that performance information about an individual collected from different perspectives and fed back to that same individual will lead to individual development" (Brutus, London \& Martineau, 1999, p. 676). However, the developmental effect of 360-degree feedback is only realized when assessee has trust in the review of the assessor. This is confirmed in a recent study by van Gennip, Segers and Tillema (2010) on the effectiveness of 360-degree feedback. The results show that the assessee's belief of psychological safety predicts significantly the assessee's perception of trust in the assessor and in turn his or her perception of performance improvement. Based on this finding, it can be assumed that when purposes of the PDP are not clear and it might be used for development as well as reward purposes, feelings of psychological safety of the assessee are under pressure.

Despite the arguments for transparency of the purpose of a PDP and for a focus on its developmental purpose, there is hardly any evidence of the influence of the purpose for which a PDP is used on its effects. Therefore, this study will examine whether the purpose of the PDP influences the extent to which professionals 
undertake learning activities and consequently their job performance. This implies this study takes the perspective of a PDP as a powerful tool to stimulate and support employees in their professional development.

\subsection{A Personal Development Plan (PDP): What?}

In an effort to make sure employees develop professionally, the interest of organizations in setting up assessment cycles, consisting of development-, follow-up-, and performance interviews, started to grow in the course of the last ten years (James \& Pedder, 2006). Similar to student evaluation or assessment, assessment within organizational settings was mostly used for purposes of accountability and promotion, finding out if and to what extent formerly defined objectives have been met. This purpose is referred to as assessment of learning or summative assessment. In the past decade, however, a vast amount of studies have evidenced that assessment is a powerful tool to support learning as well, referred to as assessment for learning or formative assessment (Assessment Reform Group, 1999; Dochy \& McDowell, 1997; Gibbs, 1999; Perrenoud, 1998).

A Personal Development Plan (PDP) is often used in organizations as part of these assessment cycles. PDPs take various forms and are presented by different synonyms: portfolio, (continuing professional) development plan, logbook or personal professional profile. The two most commonly used concepts are professional or personal development plan, and portfolio assessment. In this study, we use the term Personal Development Plan, because the term portfolio assessment carries many different connotations in organizational literature. For example, portfolio (assessment) can refer to a report system that is used for organizational accountability (e.g. Schmitz \& Schillo, 2005) or to portfolio management, that aims at the development and implementation of purchasing strategies (e.g. Gelderman, \& van Weele, 2002; Lin, Tan, \& Hsieh, 2005). Another connotation is that of portfolio work, a form of flexible self-employment in which individuals contract their skills and knowledge to different persons and organizations and develop a portfolio of job activities for themselves (Fenwick, 2006).

A PDP is a tool used to present information about the competencies the employee has been working on and is planning to further develop. It can be defined as a tool that (Brown, 1995; McMullan et al., 2003; Redman, 1994; Seng \& Seng, 1996; Smith \& Tillema, 1998; Snadden, Thomas, Griffin, \& Hudson, 1996):

- gives an overview of the competencies the employee worked on in the past and which competencies the employee is planning to work on in the future;

- $\quad$ is composed and written by the employee himself (self-direction by the employee) although the structure of the PDP is mostly fixed; 
- $\quad$ can be used as a basis/structure for conversations with the supervisor or coach who provides the employee with feedback and stimulates the employee's reflection; and

- $\quad$ serves as a decision-making tool, from planning an individual training-program (formative assessment) to assessing the suitability of a promotion (summative assessment).

Note that although the tool is often seen or labelled as an assessment tool (for learning), it is the purpose it is being put to that determines its use and whether or not it constitutes an assessment tool (Smith \& Tillema, 2003). For example, a PDP used as extended curriculum vitae is not an assessment for learning tool. In the next paragraph we will discuss the different purposes the PDP can be used for.

\subsection{Purposes of a PDP?}

In the PDP, four major questions are put forward: 'What have I done so far?', 'Where am I going?', 'How am I going?', and 'What is the next step to take?'. When a discrepancy is detected between the competencies an employee possesses and the competencies the employee should possess, it suggests that learning needs to be stimulated (Lepak, \& Snell, 1999; Hattie \& Timperley, 2007). This indicates that the core purpose of the PDP is supporting the development of the professional in order to improve performance. With respect to the purpose of a PDP, a difference has commonly been made between two broad clusters of purposes, professional development on the one hand and promotion/salary increase/selection (e.g admission to talent management programs)/accountability on the other (Smith \& Tillema, 2001). When a personal development plan is used to develop, learning takes a central part. Conversely, when a PDP is used for promotion and selection, presenting oneself is more important. Similarly, researchers investigating the purpose of performance appraisals made a distinction between using a performance appraisal to compare between and within individuals (Cleveland, Murphy, \& Williams, 1989) or for developmental and evaluative purposes (Boswell \& Boudreau, 2002). A recent literature review on the purposes of PDPs used in the workplace identified nine clusters (Beausaert et al., 2011), which can be categorized into the two broader clusters. The following purposes can be categorized as learning and development purposes:

(1) professional development;

(2) reflective learning;

(3) being coached;

(4) stimulating confidence;

(5) organizing. 
The first cluster, professional development, refers to the broader context in which the PDP is used in the workplace. It can be defined as: "A process of lifelong learning for all individuals [and teams] which enables professionals to expand and fulfil their potential" (Evans et al., 2002). Professional development incorporates personal development. "Personal development cannot be separated from professional development; each rests upon the other. Show me how well you share of yourself, understand your own personal processes and are able to communicate this to others, and I'll know how good or bad your nursing care is" (Barber, 1992: p. 309; in McMullan et al., 2003).

Many authors who mention 'professional development' as a purpose also discuss the purpose of reflective learning, the second cluster. Learning occurs more easily when reflection is stimulated, and (self-)reflection helps the employee develop his professional competencies. Reflection is defined by Daudelin (1996: p. 39) as: "the process of stepping back from an experience to ponder, carefully and persistently, its meaning to the self through the development of inferences; learning is the creation of meaning from past or current events that serves as a guide for future behaviour". In other words, reflection takes place if an employee uses theoretical insights to develop a critical view on his or her own practice, creates insights into his or her own strengths and weaknesses and identifies learning needs. Learning theories such as the experiental learning cycle of Kolb (Oslan, Kolb, Rubin, \& Turner, 2007), stress the importance of reflection as as phase in the professional learning process as well.

The third cluster, coaching, concerns the enhancement of the coaching the employee receives. Making use of a PDP formalizes the coaching the employee gets and subsequently improves the quality of the support. More specifically, making use of a PDP helps the coach to structure the coaching activities and prepare the coaching sessions. The employee's background, educational history and viewpoints are incorporated in the PDP and provide the coach with additional input for coaching activities (Firssova, 2006).

Cluster four, stimulating confidence, refers to the use of the tool as a means to boost the employee's confidence; by using a PDP, the employee becomes aware of his strengths as well as his weaknesses and is able to undertake actions to improve the latter (Snadden \&Thomas, 1998).

Cluster five, organizing, refers to the use of the instrument as an agenda, to organize the learning activities an employee has already undertaken and will undertake in the future (Wildy \& Wallace, 1998).

While professional development, reflective learning, being coached, stimulating confidence and organizing are categorized as learning and development purposes, the following purposes are grouped as promotion and selection purposes:

(6) providing evidence; 

(7) documenting;
(8) certification, selection and promotion;
(9) external mobility.

The sixth cluster, providing evidence, refers to the employee who has to document or demonstrate his competencies (cluster seven) and is considered accountable for his own learning. The difference between cluster 6 and 7 lies in the intention of the employer, who can either use the instrument to control the employee's learning (cluster 6) or not (cluster 7).

Cluster eight, certification, selection and promotion, groups purposes related to various forms of internal mobility, for example the receiving of an award. The goals recertification, licensure and tenure are included in this cluster as well. In contrast to cluster eight, the ninth cluster external mobility refers to an external selection process: the PDP is used to prepare job applications or job interviews.

On top of the distinction between these different purposes, Smith and Tillema (2001) distinguish composing a PDP on a voluntary basis from doing so on a mandatory basis. The authors found more professional development when sustained personal development plan use was voluntary than when it was mandatory (Smith \& Tillema, 2001).Other research added that staff members did not develop a [teaching] PDP unless they were obliged to (mandatory) (Bunker \& Leggett, 2004). Smith and Tillema (2003), however, found that using the PDP in a voluntary way requires a certain amount of professional maturity, as found in experienced and self-confident employees.

\subsection{Is a PDP effective for professional development?}

Although, as described above, the tool is used for various purposes, it is widely agreed that the tool is especially powerful when used to support employees' professional development (formative assessment) (Darling-Hammond \& Snyder, 2000; McDowall \& Fletcher, 2004; Smith \& Tillema, 2003). More specifically, the Beausaert et al. review study (2011) on the effectiveness of PDPs as assessment tools (a total of 54 studies were included in the review) showed that in most empirical studies PDPs are found to be effective for personal or continuing professional development purposes, for stimulating reflection and for improving the professional practice or performance. For example, Evans et al. (2002), by taking questionnaires, analyzing PDPs and in-depth interviews, studied the use of PDPs to stimulate the continuing professional development of General Practitioners (GPs). They concluded that PDPs are effective tools to stimulate the continuing professional development and personal development of GPs, on the basis that it leads to changes in patient care. Tigelaar, Dolmans, De Grave, Wolfhagen and van der Vleuten (2006) 
studied how a PDP stimulates reflection on the various aspects of teaching by analyzing the PDPs of five medical school teachers. They found that PDP assessment is effective for reflection, although the reflection is often not very profound. As a possible solution, the researchers concluded that reflection needs to be stimulated by supportive coaches. Wildy and Wallace (1998) conducted a study that researched whether using a PDP improves the professional knowledge and practice of school leaders $(N=73)$ by conducting portfolio analysis, taking a survey and observations. They found evidence that administrators who use a PDP, develop their professional knowledge and connect it with their own practice.

Despite these studies' findings, research on the effectiveness of PDPs is still scarce (e.g. Austin, Marini, \& Desroches, 2005) and the small amount of empirical research is mostly qualitative in nature and lacks an explicit description of the research method. Next, not all studies led to positive findings. For example, Bunker and Leggett (2005; in Leggett \& Bunker, 2006) argued that within the context of higher education teaching PDPs can be effective tools for stimulating promotion, but that they are not useful for encouraging reflective practice, because the PDP focuses on success and not on how to avoid failure. Orland-Barak (2005) also questioned the effectiveness of portfolios for reflection. For her research she analyzed 32 portfolios of teachers in two in-service courses for mentors of teachers in Israel. In both product $(n=20)$ and process portfolios $(n=12)$ the documentation of critical reflection was problematic. Austin, Marini and DesRoches (2005) surveyed 1415 pharmacists in Ontario, Canada. It was found that although the PDP is consistent with the idea of continuous professional development, there seems to be a lack of impact from PDPs on professional practice. Evidence was found that pharmacists did not make a close connection between documentation, self-reflection and practice improvement successfully.

How can we explain these contrasting results? A variety of problems in the PDP assessment practice might explain differences in effectiveness. Most of the aforementioned studies refer to the way the tool is being used and how the use is influenced by the way the supervisor stimulates and motivates the employee and triggers the employee's reflection with instructions and feedback. However, as Tillema (2003, p. 362) argues, the first question to address when evaluating an assessment practice, is: "What does the management want the assessment to accomplish for the organisation and what does the assessment yield?" Or, in other words: what is the purpose of the assessment activity? The study presented here addresses this first evaluative question in order to better understand the effects of the use of a PDP on professional development and performance. 


\subsection{What is the goal of this study?}

The aforementioned literature indicated different purposes that PDPs are used for and contrasting findings with regards to the effects of PDPs. The study presented here aims at better understanding if the purpose of the PDP, as perceived by the employee, influences the extent to which $s /$ he undertakes learning activities and how this in turn leads to improved performance, for which learning is assumed.

The central research questions are: (1) which purposes of a PDP, as perceived by the employee, predict the undertaking of learning activities and performance significantly positively? and, (2) which is the most powerful predictor of undertaking learning activities and performance? Based on the aforementioned literature, the following working hypotheses are formulated:

$\mathrm{H} 1$. Perceiving the PDP as a learning and development tool will predict the employee's undertaking of learning activities and the employee's performance significantly positively.

$H 2$. Perceiving the PDP as a promotion and selection tool will predict the employee's undertaking of learning activities and the employee's performance significantly negatively.

H3. In the case both learning/development and promotion/selection purposes are taken into account perceiving the PDP as a learning and development tool will be the most powerful predictor of the employee's undertaking of learning activities.

\section{Method}

\subsection{The participating organizations and their employees}

Organization 1. The participants are employees of a regional Dutch governmental office. It employs 1400 people in 5 different offices, located in 4 different cities. Despite the relatively large amount of employees working in different departments, the organization is perceived as a mid-sized organization. The average age of the employees was 49 in 2009 with a very low turn-around of employees.

The employees of the governmental office annually undergo an assessment cycle. The assessment cycle consists of a performance interview, a development interview and an assessment interview with the supervisor. During the assessment process the supervisor and the employee can make use of two different supporting instruments, i.e. the 'Knowledge-ID' and the 'Evaluation-form'. While the Knowledge-ID provides the supervisor with an extended CV of the employee's previous learning experiences, the Evaluation-form not only looks backward, but also determines the competencies an employee still needs to develop. One year ago the central HR-office of the governmental offices in the Netherlands decided to imple- 
ment a new instrument: the Personal Development Plan (PDP). The PDP integrates the Knowledge-ID and the Evaluation-form by listing the competencies the employee still needs to develop (looking forward) through evaluating the strengths and weaknesses of the employee (looking back). The tools are not used as strictly by every supervisor and the HR department as well as the remarks of the employees on the questionnaire indicate that the tool is not well implemented. When we use the term Personal Development Plan in regard to Organization 1 in this article, we refer to all three, meaning the Knowledge-ID, the Evaluation-form and/or the PDP.

Out of the 1400 employees that were contacted, a total of 286 (response rate 20\%, 187 men and 72 female; 27 missing values) participated in the research, spread over at least six different departments and four office locations. Of the 234 employees who provided us with their highest education level, 27 studied WO (university), 81 HBO (non-academic higher education), 16 VWO (academicallyoriented secondary education), 47 HAVO (higher secondary education), 55 MBO (secondary vocational education) and 8 VMBO (lower secondary vocational education). The average employee was 50 years old (SD =7). The average number of years of experience in the organization was 22 years $(S D=13)$ and the average number of years of experience in the current role was 11 years (SD =10). These numbers are in line with the statistics that are available for the total group of employees.

Organization 2. The participants are experts from an international organization that specializes in medical technology. The organization employs approximately 38.000 people in 120 countries. The office that participated employs 200 in total. The assessment cycle in this organization is similar to the one in Organization 1. First, over the course of one year the personal objectives of the employee are determined. Next, the employee has to fill out a performance measure and a PDP; a select group of employees also works on a talent portfolio. The three instruments are linked to each other and each is discussed during a meeting. We treat the three instruments as one. Furthermore, evaluating the effectiveness of the PDP, we do not only refer to the tool, but also to the meetings in which the PDP is discussed. In contrast to Organization 1 the HR indicates that the tool is well implemented and is used strictly.

Out of the 200 employees that were contacted, a final total of 81 (28 men and 49 women; 4 missing values) participated in the research, a response rate of $41 \%$. Of those 81 employees, 7 had a secondary education degree, 20 had a professional bachelor's degree and 50 had an academic master's degree or a PhD. The average age was 38. The number of years of experience in the current role was on average 8 years. 


\subsection{Measures}

The different variables were measured by a questionnaire. The questionnaire consisted of three sections. Section 1 measured the perceived purpose of the PDP. Section 2 measured the dependent variables: Learning activities undertaken and Performance. Section 3 asked for information on the employee's background (highest level of education (certificate), gender and experience in the current role). With the exception of the items concerning the background information, all questions were answered making use of a 5-point Likert scale, going from totally agree to totally disagree or from always to never (undertaking learning activities).

The perceived purpose of the PDP. To measure the perceived purposes of the PDP, the Perceived Nature of the Assessment Goals Questionnaire (PNAGQ) was developed, based on a literature review on PDP assessment in organizations (Beausaert et al., 2011). The questionnaire asks the employees to rate the perceived PDP purposes on a 5-point Likert scale concerning the strength of its pursuit going from 'totally agree' to 'totally disagree'. The questionnaire questions the following 15 purposes of a PDP: personal or professional development, self-assessment, stimulating learning and reflection, to deliver evidence to my supervisor, to demonstrate or document, obtaining a certificate or a license, preparing an external job interview, accreditation/accomplishing the organization's standards, selecting/making promotion, receiving coaching, stimulating collaboration with colleagues, motivating, organize oneself/keeping an agenda of learning activities, stimulate confidence, and stimulating the development of the organization.

The Perceived Nature of the Assessments Goals Questionnaire (PNAGQ) was validated in two steps. To explore the optimal factorial structure of the questionnaire an exploratory factor analysis was conducted on the data collected in Organization 1 ( $n=286$ ). Next, a confirmatory factor analysis was performed on the data collected at Organization $2(n=81)$ to determine the robustness of the factor structure across samples.

First, to assess the validity of the two distinct groups of purposes, learning and development purposes on the one hand and promotion and selection purposes on the other hand, an exploratory factor analysis was performed. In contrast to our expectations, the factor analysis with direct oblimin rotation indicated the existence of three factors with item loads of .46 and more. The first component had an eigenvalue of 7.88 (corresponding to $53 \%$ of the explained variance), the second component had an eigenvalue of 1.49 (corresponding to $10 \%$ of the explained variance) and the third component had an eigenvalue of 1.04 (corresponding to $7 \%$ of the explained variance). The items stimulating collaboration with colleagues, stimulating the development of the organization, accreditation/ accomplishing the organization's standards, motivating, and stimulating confidence loaded on the first 
component, which was labeled the organizational learning and development purposes scale ( 5 items). Preparing an external job interview, to demonstrate or document, obtaining a certificate or a license, to deliver evidence to my supervisor, and selecting/making promotion loaded on the second component. The component was labeled the selection and promotion purposes scale ( 5 items). The items personal or professional development, self-assessment, stimulating learning and reflection and organize oneself/keeping an agenda of learning activities loaded on the third component which was labeled the personal learning and development purposes scale (4 items). One item had a low loading (.397; "Receiving coaching") and as a result did not load on any factor. The Cronbach's alphas were .91 for the personal learning and development purposes scale, .90 for the organizational learning and development purposes scale and .80 for the promotion and selection purposes scale.

Second, in order to test the robustness of the structure of the three components of the PNAGQ that was found in the exploratory factor analysis, a confirmatory factor analysis (CFA) was performed. The model showed a poor fit to the observed data as suggested by the goodness-of-fit-indices $\left(\chi^{2}=177.19\right.$; $S R M R=0.087$, RMSEA $=0.13 ; \mathrm{CFI}=0.82$ ). Next, based on the largest standardized residuals, the LM test and the Wald test, the hypothesized model was optimized. Two items (organizing oneself/keeping an agenda of learning activities, and stimulating collaboration with colleagues) had to be deleted. The two items were deleted especially because of too many cross loadings, and, the items had already demonstrated a lower factor loading in the exploratory factor analysis. Furthermore, in line with the content of the items, the correlation between two items loading on the same factor was accepted, namely between "to demonstrate or document" and "to deliver evidence to my supervisor" and also between "obtaining a certificate or a license" and "preparing an external job interview". This resulted in an acceptable moderate model fit $\left(\chi^{2}=85.36, p=.001 ;\right.$ SRMR $=.06$; RMSEA $\left.=0.10 ; \mathrm{CFI}=0.92\right)$. The Cronbach's alphas were .89 for the personal learning and development purposes scale (3 items), .88 for the organizational learning and development purposes scale (4 items) and .80 for the promotion and selection purposes scale ( 5 items).

Undertaking learning activities. The different types of learning activities undertaken were evaluated with the Learning Activities Scale (6 items). The scale was developed based on a literature review about the effects of PDPs and measures to which extent employees undertake learning activities as a result of working with a PDP on a behavioral level. More specifically, on a 5-point Likert scale going from never to always, the questionnaire asked how often different types of learning activities were undertaken because of the PDP (i.e. trainings, courses, workshops, conferences, intervisions, supervisions, internships and/or self-study). 
A maximum likelihood test on the total data file resulted in one factor with item loadings of .67 and higher. The component had an eigenvalue of 3.95 (corresponding to $66 \%$ of the explained variance). All items that tagged the scale Undertaking learning activities loaded on this component. The Cronbach's alpha was .90.

Performance. To measure the perceived performance we adapted the Output of transfer behavior scale of Xiao (1996), consisting of 6 items. Originally the scale was used to measure the effects of transfer of training. We adapted the questionnaire in order to measure the effects of an assessment tool, the PDP on the employee's performance. For example, the item "Using the new KSA has helped me improve my work" was translated into "Using a PDP has helped me to improve my work".

A maximum likelihood test on the total datafile resulted in one factor with item loadings of .88 and higher. The component had an eigenvalue of 5.12 (corresponding to $85 \%$ of the explained variance). The Cronbach's alpha was .97 .

For an overview of the different scales, example items, and Cronbach's alphas, we refer to Table 1.

Table 1

Overview of the different scales and their descriptives

\begin{tabular}{|c|c|c|c|}
\hline Scale & $\mathrm{N}$ & $\alpha$ & Example items \\
\hline $\begin{array}{l}\text { The perceived nature of the } \\
\text { purposes }\end{array}$ & & & $\begin{array}{l}\text { Indicate on a Likert scale going from } 1 \text { to } 5 \text { in which } \\
\text { way your organization is striving for the following goals } \\
\text { by implementing PDPs: }\end{array}$ \\
\hline $\begin{array}{l}\text { Personal learning and devel- } \\
\text { opment purposes }\end{array}$ & 3 & .89 & Stimulate refletion or learning. \\
\hline $\begin{array}{l}\text { Organizational learning and } \\
\text { development purposes }\end{array}$ & 4 & .88 & Stimulating collaboration with colleagues. \\
\hline $\begin{array}{l}\text { Promotion and selection } \\
\text { purposes }\end{array}$ & 4 & .80 & To delivering evidence to my supervisor. \\
\hline \multicolumn{4}{|l|}{ Outcome variables } \\
\hline Undertaking learning activities & 6 & .90 & $\begin{array}{l}\text { Because of using a PDP I look up things in books, } \\
\text { journal or on the internet. }\end{array}$ \\
\hline Performance & 6 & .97 & $\begin{array}{l}\text { Since I am using a PDP and have related meetings, the } \\
\text { quality of my work improved. }\end{array}$ \\
\hline
\end{tabular}

\subsection{Procedure}

Both organizations were contacted and invited to participate in the research. After an introductory meeting, the questionnaire was adapted to the specific setting of the organization in cooperation with a HR consultant. Finally the questionnaire was distributed by the HR-consultant via email, with a link to the questionnaire. To guarantee the anonymity of the employees, the data were gathered immediately by the software (NetQ). To increase the response rate, the employees received one reminder via email and in Organization 1 a weekly newsletter as well. 


\subsection{Data analysis}

Firstly, descriptives of the different variables in the study were calculated. Secondly, correlation analysis explored the relation between the perceived purpose components (personal learning and development purposes, organizational learning and development purposes, and promotion and selection purposes) and the outcome variables (undertaking learning activities and performance). Thirdly, hierarchical regression-analyses were conducted to identify whether the independent variables (perceived learning and development purposes, perceived promotion and selection purposes) predicts the employee's undertaking of learning activities and performance. Fourthly, a hierarchical regression analysis was conducted, including both purposes at the same time, in order to determine the strongest predictor of undertaking learning activities and performance. Additional analyses of covariance (ANCOVAs) are performed in order to look into the interaction effects of the different purposes scales.

\section{Results}

\subsection{Preliminary analysis}

Table 2

Correlations

\begin{tabular}{|c|c|c|c|c|c|c|c|}
\hline Variable & $\mathrm{M}$ & SD & 1 & 2 & 3 & 4 & 5 \\
\hline $\begin{array}{l}\text { 1. Personal learning and devel- } \\
\text { opment purposes }\end{array}$ & 2.60 & 1.05 & - & & & & \\
\hline $\begin{array}{l}\text { 2. Organizational learning and } \\
\text { development purposes }\end{array}$ & 2.38 & 1.01 & $.68^{* *}$ & - & & & \\
\hline $\begin{array}{l}\text { 3. Promotion and selection } \\
\text { purposes }\end{array}$ & 2.11 & .87 & $.44^{* *}$ & $.52 * *$ & - & & \\
\hline $\begin{array}{l}\text { 4. Undertaking learning activi- } \\
\text { ties }\end{array}$ & 2.62 & .91 & $.13^{*}$ & $.23 * *$ & $.34^{* *}$ & - & \\
\hline 5. Performance & 2.58 & .84 & $.34^{* *}$ & $.36 * *$ & $.29 * *$ & $.34 * *$ & - \\
\hline
\end{tabular}

Note: ${ }^{* *} p<.01{ }^{*} p<.05$.

Table 2 shows the means and the standard deviations and correlations between the three subscales of the PNAGQ (personal learning and development purposes, organizational learning and development purposes and promotion and selection purposes) and the two outcome variables (undertaking learning activities and performance). The results indicate that the three different purposes correlate significantly positively with Undertaking learning activities and Performance. Next, the purposes correlate significantly positively and Undertaking learning activities and Performance correlate significantly positively as well. 


\subsection{Hypothesis 1: Perceiving the PDP as a learning and development tool will predict the employee's undertaking of learning activities and the employee's performance significantly positively}

To examine the effect of the perceived individual (Table 3a) and organizational (Table $3 b$ ) learning and development purposes on the employee's undertaking of learning activities and performance, a hierarchical regression analysis was executed. In Step 1 the background variables organization, highest level of education (certificate), gender and experience in the current role were entered. In Step 2 the purpose variable was entered. The findings indicate that there is a difference between both participating organizations in the undertaking of learning activities. Next, the personal as well as organizational learning and development purposes predict the employee's undertaking of learning activities $(B=.18, p<.001$ and $B=$ $.20, p<.001)$ and performance $(B=.36, p<.001$ and $B=.35, p<.001)$ significantly positively, which confirms Hypothesis 1 . In other words, if the employee perceives the PDP as a learning and development tool, the employee undertakes learning activities and experiences improvement of his or her performance. 


\section{Chapter 4}

Table $3 a$

Hierarchical regression analysis of the independent personal learning and development purposes scale on the dependent variables (undertaking learning activities and performance)

\begin{tabular}{|c|c|c|c|c|}
\hline & \multicolumn{2}{|c|}{$\begin{array}{l}\text { Undertaking learning } \\
\text { activities }\end{array}$} & \multicolumn{2}{|c|}{ Performance } \\
\hline & $\beta$ & $\Delta \mathrm{R}^{2}$ & $\beta$ & $\Delta R^{2}$ \\
\hline \multicolumn{5}{|l|}{ Step 1} \\
\hline Organization & $.37 * * *$ & & .04 & \\
\hline Education & -.09 & & -.08 & \\
\hline Gender & .02 & & .01 & \\
\hline \multirow[t]{2}{*}{ Experience in role } & -.06 & & -.03 & \\
\hline & & $.20 * * *$ & & .01 \\
\hline \multicolumn{5}{|l|}{ Step 2} \\
\hline Organization & $.39 * * *$ & & .07 & \\
\hline Education & $-.13 *$ & & $-.15^{*}$ & \\
\hline Gender & .01 & & -.01 & \\
\hline Experience in role & -.04 & & .00 & \\
\hline \multirow[t]{2}{*}{ Personal learning and development purposes } & $.18^{* * *}$ & & $.36 * * *$ & \\
\hline & & $.03 * * *$ & & $.12 * * *$ \\
\hline
\end{tabular}

Note. The reported regression coefficients are standardized coefficients.

$* \mathrm{p}<.05 . * * \mathrm{p}<.01 . * * \mathrm{p} p<.001$.

Table 3b

Hierarchical regression analysis of the independent organizational learning and development purposes scale on the dependent variables (undertaking learning activities and performance)

\begin{tabular}{|c|c|c|c|c|}
\hline & \multicolumn{2}{|c|}{$\begin{array}{l}\text { Undertaking learning } \\
\text { activities }\end{array}$} & \multicolumn{2}{|c|}{ Performance } \\
\hline & $\beta$ & $\Delta \mathrm{R}^{2}$ & $\beta$ & $\Delta R^{2}$ \\
\hline \multicolumn{5}{|l|}{ Step 1} \\
\hline Organization & $.37 * * *$ & & .04 & \\
\hline Education & -.09 & & -.08 & \\
\hline Gender & .02 & & .01 & \\
\hline \multirow[t]{2}{*}{ Experience in role } & -.06 & & -.03 & \\
\hline & & $.20 * * *$ & & .01 \\
\hline \multicolumn{5}{|l|}{ Step 2} \\
\hline Organization & $.37 * * *$ & & .03 & \\
\hline Education & -.09 & & -.08 & \\
\hline Gender & .00 & & -.01 & \\
\hline Experience in role & -.06 & & -.03 & \\
\hline \multirow{2}{*}{$\begin{array}{l}\text { Organizational learning and development } \\
\text { purposes }\end{array}$} & $.20 * * *$ & & $.35 * * *$ & \\
\hline & & $.04 * * *$ & & $.12 * * *$ \\
\hline
\end{tabular}

Note. The reported regression coefficients are standardized coefficients.

${ }^{*} p<.05 \quad .{ }^{* *} p<.01 .{ }^{* * *} p<.001$. 


\subsection{Hypothesis 2: Perceiving the PDP as a selection and certification tool will predict the employee's undertaking of learning activities and the employee's performance significantly negatively}

To examine the effect of the perceived promotion and selection purposes on the employee's undertaking of learning activities and performance, a hierarchical regression analysis was executed (Table $3 \mathrm{c}$ ). Similar as in 3.2. the background variables organization, highest level of education (certificate), gender and experience in the current role were entered in Step 1. In Step 2 the purpose variable was entered. Again the findings indicate that there is a difference between both participating organizations in the undertaking of learning activities. Next, in contrast to our expectations, the Promotion and selection purposes also predict the undertaking of learning activities $(B=.16, p<.01)$ and the employee's performance $(\theta=.32$, $p<.001)$ significantly positively, which is not in line with Hypothesis 2 . Hypothesis 2 is not confirmed. The results show that perceiving the PDP as a promotion and selection tool leads towards the undertaking of learning activities and a better performance.

Table 3c

Hierarchical regression analysis of the independent Promotion and selection purposes scale on the dependent variables (undertaking learning activities and performance)

\begin{tabular}{|c|c|c|c|c|}
\hline & \multicolumn{2}{|c|}{$\begin{array}{l}\text { Undertaking learning } \\
\text { activities }\end{array}$} & \multicolumn{2}{|c|}{ Performance } \\
\hline & $\beta$ & $\Delta \mathrm{R}^{2}$ & $\beta$ & $\Delta R^{2}$ \\
\hline \multicolumn{5}{|l|}{ Step 1} \\
\hline Organization & $.37 * * *$ & & .04 & \\
\hline Education & -.09 & & -.08 & \\
\hline Gender & .02 & & .01 & \\
\hline \multirow[t]{2}{*}{ Experience in role } & -.06 & & -.03 & \\
\hline & & $.20 * * *$ & & .01 \\
\hline \multicolumn{5}{|l|}{ Step 2} \\
\hline Organization & $.30 * * *$ & & -.11 & \\
\hline Education & -.10 & & -.10 & \\
\hline Gender & .01 & & -.01 & \\
\hline Experience in role & -.04 & & .00 & \\
\hline \multirow[t]{2}{*}{ Promotion and selection purposes } & $.16^{* *}$ & & $.32 * * *$ & \\
\hline & & $.02 * *$ & & $.08 * * *$ \\
\hline
\end{tabular}

Note. The reported regression coefficients are standardized coefficients.

$* \mathrm{p}<.05 \quad . * * \mathrm{p}<.01 . * * * \mathrm{p}<.001$. 


\subsection{Hypothesis 3: In the case both learning/development and promo- tion/selection purposes are taken into account, perceiving the PDP as a learning and development tool will be the most powerful predictor of the employee's undertaking of learning activities}

In order to determine which purpose is the most powerful predictor of the employee's undertaking of learning activities and performance, we conducted two hierarchical regression analyses, one for each dependent variable (Table 4). In Step 1 we again entered the background variables organization, highest level of education (certificate), gender and years of experience in the current role. In Step 2 the two learning and development purpose variables were entered with alternatively undertaking learning activities and performance as the dependent variables. Finally, in Step 3 the promotion and selection purpose variable was entered. The table shows that perceiving organizational learning and development purposes is the strongest predictor of undertaking learning activities $(\beta=.14, p<.05)$, while both perceiving personal $(B=.18, p<.05)$ and organizational $(B=.18, p<.05)$ learning and development purposes are the strongest predictor of an improved performance. No additional variance is explained by perceiving promotion and selection purposes. In conclusion, if you want employees to undertake learning activities and deliver a higher-quality performance when using a PDP, the tool should be introduced as a learning and development tool.

Additional analyses of covariance (ANCOVAs) were performed for both outcome variables. We analyzed the main effects of the three purposes discerned (the personal learning and development purposes, the organizational learning and development purposes, the promotion and selection purposes) and their interaction effects with organization as a covariate. The analyses for the outcome variable the undertaking of learning activities confirmed the results of the regression analysis reported above. In addition, no interaction effects were found. The results of the four interaction analyses indicated that there is no significant effect of the interaction between the different purposes of the PDP on the extent to which employees undertake learning activities. (personal learning and development purposes* organizational learning and development purposes, $F(40,80)=1.02, p=0.46$; personal learning and development purposes*promotion and selection purposes, $F(52,80)=$ 1.03, $p=0.45$; organizational learning and development purposes*promotion and selection purposes, $F(55,80)=1.01, p=0.48$; personal learning and development purposes* organizational learning and development purposes*promotion and selection purposes, $F(1,80)=2.10, p=0.15)$. These results were confirmed when conducting ANCOVA with performance as outcome variable. 
Table 4

Hierarchical regression analysis of all purpose components on the dependent variables (undertaking learning activities and performance)

\begin{tabular}{|c|c|c|c|c|}
\hline & \multicolumn{2}{|c|}{$\begin{array}{l}\text { Undertaking learning } \\
\text { activities }\end{array}$} & \multicolumn{2}{|c|}{ Performance } \\
\hline & $\beta$ & $\Delta R^{2}$ & ß & $\Delta R^{2}$ \\
\hline \multicolumn{5}{|l|}{ Step 1} \\
\hline Organization & $.37 * * *$ & & .04 & \\
\hline Education & -.09 & & -.08 & \\
\hline Gender & .02 & & .01 & \\
\hline \multirow[t]{2}{*}{ Experience in role } & -.06 & & -.03 & \\
\hline & & $.20 * * *$ & & .01 \\
\hline \multicolumn{5}{|l|}{ Step 2} \\
\hline Organization & $.38 * * *$ & & .05 & \\
\hline Education & -.11 & & $-.12 *$ & \\
\hline Gender & .00 & & -.01 & \\
\hline Experience in role & -.05 & & -.01 & \\
\hline Personal learning and development purposes & .07 & & $.22 * *$ & \\
\hline \multirow{2}{*}{$\begin{array}{l}\text { Organizational learning and development } \\
\text { purposes }\end{array}$} & $.15^{*}$ & & $.20 * *$ & \\
\hline & & $.04 * * *$ & & $.14 * * *$ \\
\hline \multicolumn{5}{|l|}{ Step 3} \\
\hline Organization & $.36 * * *$ & & .00 & \\
\hline Education & -.11 & & $-.12^{*}$ & \\
\hline Gender & .00 & & -.02 & \\
\hline Experience in role & -.05 & & -.01 & \\
\hline Personal learning and development purposes & .06 & & $.18^{*}$ & \\
\hline $\begin{array}{l}\text { Organizational learning and development } \\
\text { purposes }\end{array}$ & $.14^{*}$ & & $.18^{*}$ & \\
\hline \multirow[t]{2}{*}{ Promotion and selection purposes } & .04 & & .10 & \\
\hline & & .00 & & .01 \\
\hline
\end{tabular}

Note. The reported regression coefficients are standardized coefficients.

${ }^{*} p<.05 \quad .{ }^{* *} p<.01 .{ }^{* * *} p<.001$.

\subsection{ANOVA: Differences between organizations}

The regression analysis indicated the effect of the organization on the undertaking of learning activities as a result of using a PDP. In an effort to better understand the powerful effect of the organization on the undertaking of learning activities, Table 5 illustrates the descriptives (means and standard deviations) for both groups and reports an analysis of variance (ANOVA) for the three purpose components and both outcome variables (undertaking learning activities and performance). The results of the ANOVA indicate that the employees in Organization 1 perceive the PDP as a tool for personal learning and development to a greater extent than the employees of Organization 2. These results also suggest that, in comparison with Organization 1, the tool in Organization 2 is more used as a promotion and selec- 
tion instrument. Moreover, more learning activities are undertaken in organisation 2 than in organisation 1, although there are no significant differences in performance.

Table 5

Analysis of variance between Organization 1 and Organization 2

\begin{tabular}{|c|c|c|c|c|c|c|c|}
\hline \multirow[t]{2}{*}{ Variable } & \multicolumn{3}{|c|}{ Organization $1(\mathrm{~N}=286)$} & \multicolumn{3}{|c|}{ Organization $2(\mathrm{~N}=81)$} & \multirow[t]{2}{*}{$\mathrm{F}$} \\
\hline & $\mathrm{N}$ & $M$ & SD & $\mathrm{N}$ & $M$ & SD & \\
\hline $\begin{array}{l}\text { Personal learning and } \\
\text { development purposes }\end{array}$ & 286 & 2.67 & 1.10 & 81 & 2.36 & .81 & $5.43^{*}$ \\
\hline $\begin{array}{l}\text { Organizational learning } \\
\text { and development pur- } \\
\text { poses }\end{array}$ & 286 & 2.35 & 1.06 & 81 & 2.48 & .81 & 1.10 \\
\hline $\begin{array}{l}\text { Promotion and selection } \\
\text { purposes }\end{array}$ & 286 & 1.90 & .82 & 81 & 2.86 & .60 & $97.60 * * *$ \\
\hline $\begin{array}{l}\text { Undertaking learning } \\
\text { activities }\end{array}$ & 286 & 2.41 & .87 & 81 & 3.36 & .65 & $84.99 * * *$ \\
\hline Performance & 286 & 2.54 & .86 & 81 & 2.74 & .78 & 3.62 \\
\hline
\end{tabular}

Note. ${ }^{*} p<.05 .{ }^{* *} p<.01 .{ }^{* * *} p<.001$.

\section{Conclusion and discussion}

Personal Development Plans (PDPs) are taking an increasingly central role in the assessment cycles in organizations. A PDP is a tool used to present information about the competencies the employee has been working on and is planning to further develop. The tool can be used for different purposes. A difference is commonly established between professional development and promotion/salary raise/selection/accountability (Smith \& Tillema, 2001). When a personal development plan is used for professional development, learning takes a central role. Conversely, when a PDP is used for promotion and selection, presenting oneself is more important.

Although there is a trend towards using the tool for performance appraisal, the tool is especially powerful to support employees' professional development (Smith \& Tillema, 2003). This study addresses whether the purposes for which the PDP is used affects the extent to which employees undertake learning activities and consequently their performance. Based on the regression analysis results, we can conclude that perceiving the PDP either as a learning and development tool or as a promotion and selection tool positively predicts the undertaking of learning activities and the employee's performance. However, subsequent hierarchical linear regression analyses, including the three discerned purposes of a PDP, indicated that the most powerful predictor of undertaking learning activities and a high-quality performance are (organizational and personal) learning and development purposes 
and not promotion and selection purposes. These results show that when an employee perceives the PDP as a tool for learning and development he or she is more likely to undertake learning activities and in turn perform better as a result of using a PDP than when the assessment tool is perceived as a promotion and selection instrument. The results are in line with previous and similar research conducted by Beck et al. (2005) who compared the effects of formative and summative portfolios on the professional outcomes of pre-service and beginning teachers. They concluded that portfolios focusing on teacher development better supported professional outcomes than did the summative accountability portfolio. The researchers even concluded that the tool should not be used for the summative accountability of teachers. Similarly, Tillema (2001) found that a strong preoccupation with performance appraisal (summative assessment) may counter learning and development purposes. Smith and Tillema (2003) stated that "The higher the stakes of assessment of the PDP [portfolio], the less valuable it becomes for professional development purposes". Similarly, researchers investigated the different effects of process focus appraisals and exclusive results-oriented appraisals on expectations of performance improvement and found more positive effects of process focus appraisals (Lam \& Schaubroeck, 1999). However, the results are in contrast with the research of Tigelaar, Dolmans, Wolfhagen and van der Vleuten (2004) who concluded on the basis of nine interviews with portfolio experts that portfolios can be used for both learning and selection purposes; focusing on learning first and shifting to selection purposes later, for example because the employee's motivation to learn will be triggered. Boswell and Boudreau (2002) who researched the separate use of developmental and evaluative performance appraisal interviews also found contrasting results. They found that the employees in the separated performance appraisal group (versus the traditional performance appraisal group) intended to undertake less development activities in the future.

In addition, the hierarchical regression analyses show the organization has a strong predictive power in terms of undertaking learning activities. The results of the ANOVA indicate that while employees in Organization 1 perceive the tool as a tool for personal and organizational learning and development first and foremost, the employees in Organization 2 especially perceive the tool as a tool for promotion and selection. However, the employees in Organization 2 undertake significantly more learning activities than those in Organisation 1 . The differences between the organisational culture of both organizations might explain these results. Martins \& Terblanche (2003) for example make a distinction between organizations according to the competitive behaviour, vision and mission of the organization, the organizational structure and the freedom and flexibility. First, Organization 1 is a non-profit organization. This means that the organization does not compete with other companies and therefore is not forced to keep up with the newest developments (Martins \& Terblanche, 2003). Consequently in this type of organizations 
learning and development do not play a pivotal role. Next, career perspectives are limited and competition between employees is scarce in Organization 1, while Read (1996) indicates that competitiveness stimulates knowledge creation and continuously updating of knowledge. Second, oral communication with the HR department as well as the remarks of the employees on the questionnaire indicated that in Organization 1 the tool is not closely related to the mission and vision of the organization and that whether or not one uses the tool has no significant consequences. However, HR tools that are not well imbedded within the mission and strategy of an organization lack affectivity (Wintermantel \& Mattimore, 1997). Finally, governmental offices are more hierarchical, stable and bureaucratic organizations in which employees are less stimulated to autonomously use their knowledge and skills in a dynamic way, again leading to little innovation and therefore in turn jeopardizes the importance of the role of continuous learning and development (Martins \& Terblanche, 2003). More specifically, Cleveland and Shore (1992) refer to the age of the employees. The average age of the employees in Organization 1 is 49 . This means that a large group of the employees could be considered experts who are no longer interested in learning and developing systematically.

In contrast, Organization 2 is a profit organization. This entails that Organization 2 is a more dynamic organization which has to keep up with the newest developments in order to compete with other companies. Furthermore, Organization 2 is situated in the medical sector in which knowledge evolves fast. Next, the PDP is part of a better implemented assessment system in which it performs a central task; the use of the tool heavily influences whether or not an employee is selected for promotion. Finally, this organization has a younger staff; the average age is 38 . It is likely that a larger group of novices is interested in learning and developing and consequently making promotion or working on their employability. In sum, Organization 2 has a more competitive external and internal environment, uses HR tools which are better imbedded within the strategy of the organization and has a younger staff. These characteristics of the organizational culture of Organization 2 support innovation and creation which in turn enhances attention for continuous learning and development.

\subsection{Limitations and future research}

When we interpret the previous findings, we need to take into account some limitations which future research might be faced with and should address. A first limitation concerns the generalization of the research. This research incorporated results from two organizations. Future research should further cross-validate our findings across organizations and sectors. Before organizations are chosen, it is important to carefully explore the way the PDP is implemented. Often the manag- 
ers will describe the HR process differently than the way the PDP practice is actually organized.

Furthermore, in addition to the purpose of the PDP, there are various personal, environmental and career related conditions which can influence the PDP practice and in turn the undertaking of learning activities and the employee's performance. For example, the employee's motivation, self-efficacy, need for reflection and age (Beausaert et al., 2011). Moreover, with respect to environmental conditions, how the PDP is introduced and supported by the company, the learning culture and the supervisor can influence the effects of the tool significantly. More specifically, the supervisor - who often communicates the purpose, coaches the process and motivates the employee - and the feedback given by the supervisor play a pivotal role. The feedback stimulates the employee's reflection and is essential to let the employee develop (Beausaert et al, 2011; Smith \& Tillema, 2003). Next, changes within the environment of the employee such as reorganizations, technological innovations and new products, are important triggers for employees to reflect. Finally, pending on the career phase of the employee, s/he may feel a bigger need for reflection. For example, an employee who is retiring may feel a less strong need for reflection than an employee who just started working for the organization.

With respect to the methodology used, the data in this study are based on selfreports by employees. For the measurement of the purposes of a PDP, self-report measures are the most valid method. There is a significant amount of evidence that the assessment practice influences the employee's learning outcomes via the employee's perception of this practice (e.g. Biggs, 2003; Prosser \& Trigwell, 1999). However, questioning employees on their learning activities and their performance can induce socially desirable answers. Using 360-degree assessment, including peers and supervisors of the employees questioned, is advisable in future research. Finally, because of this study's cross-sectional design, changes over time cannot be determined; consequently it does not allow us to draw conclusions about causality.

Finally, for deepening our understanding of how the PDP practice affects the employees' perceptions of the tool, we recommend the additional use of qualitative research methods such as interviews.

\subsection{Practical implications}

This study has a few implications for human resource development in organizations. First, in order to make employees undertake learning activities and improve their performances by using a PDP, introducing and using the PDP as a tool for learning and development is the most effective. This means that the tool is presented as a learning and development tool and used to stimulate the employee's development. In order for this to work the supervisor needs to make the learning and development purpose of the PDP explicit to the employee and support the use 
as a learning tool with appropriate guidance. Furthermore, the supervisor should stimulate the employee to not only look back on what he or she has already learned, but also explicitly pay attention to what still needs to be learned in the future.

Perceiving the tool as a promotion and selection tool, however, also predicts the undertaking of learning activities and employees' performance positively. This supports the opinion of some authors who indicated that the PDP can be used for both purposes (e.g. Snyder, Pippincott \& Bower, 1998). Nevertheless, perceiving learning and development purposes is a stronger predictor than perceiving promotion and selection purposes. This leads to the question: How to balance between promotion and selection purposes on the one hand and learning and development purposes on the other, knowing that learning and development purposes are stronger predictors of undertaking learning activities and performance? The following suggestions might help to answer that question: (1) Keep learning and development interviews separate from performance interviews. The performance interviews can still be based on a selection of evidence which is collected in the PDP and which is used for learning and development interviews. (2) Similarly, have the two different kinds of interviews conducted by a different person in order to split up both purposes completely. The interviews with learning and development purposes should be conducted by a coach, while the interviews with promotion and selection purposes should be performed by the supervisor. In practice the PDP interviews and the appraisal interviews are mostly conducted by one and the same supervisor. (3) Make a distinction between the criteria used for discussing the PDP during the learning and development interviews and the criteria used during performance interviews. While growth indicators, making the growth in competencies visible, should be used in the first type of interviews, attainment indicators, pointing out if the specific level of proficiency that was agreed upon before is reached, should be used in the performance interviews. (4) Make the difference between the two types of interviews and the role of the PDP within those interviews clear by referring to the link between the different interviews and other HRD-activities. For example, the link between discussing a PDP during a learning and development interview on the one hand and training on the other hand should be clearly made. (5) Make a distinction between the different parties involved in the learning and development interviews and the parties involved in the promotion and selection interviews. For instance, colleagues can be a fruitful source of feedback in the employee's developing process. Often colleagues work closer with the employee than the supervisor and they are more aware of the employee's weaknesses and strengths. (6) Make the difference between the two types of interviews clear by referring to possible follow-up activities after the PDP interview. 


\section{References}

Assessment Reform Group. (1999). Position paper on assessment for learning from the Third International Conference on Assessment for Learning, Dunedin, New Zealand, March 2009.

Austin, Z., Marini, A., \& Desroches, B. (2005). Use of a learning portfolio for continuous professional development: A study of pharmacists in Ontario (Canada). Pharmacy Education, 5, 175-181.

Beausaert, S., Segers, M., van der Rijt, J., Gijselaers, W. (2011). The use of Personal Development Plans in the workplace: A literature review. In P. van den Bossche, W. Gijselaers, \& R. Milter (Eds.), Building learning experiences in a changing world, Advances in Business Education and Training III (pp. 235-265). Dordrecht: Springer.

Beck, R.J., Livne, N.L., \& Bear, S.L. (2005). Teachers' self-assessment of the effects of formative and summative electronic portfolios on professional development. European Journal of Teacher Education, 28, 221-244.

Biggs, J. (2003). Teaching for quality learning at university. Maidenhead: McGraw-Hill Education.

Boswell, W.R., \& Boudreau, J.W. (2002). Separating the developmental and evaluative performance appraisal uses. Journal of Business and Psychology, 16, 391-412.

Brown, R. (1995). Portfolio development and profiling for nurses, $2^{\text {nd }}$ edn. Lancaster: Quay Publications.

Brutus, S., London, M., \& Martineau, J. (1999). The impact of 360-degree feedback on planning for career development. Journal of Management Development, 18, 676-693.

Bullock, A., Firmstone, V., Frame, J., \& Bedward, J. (2007). Enhancing the benefit of continuing professional development: A randomized controlled study of personal development plans for dentists. Learning in Health and Social Care, 6 (1), 14-26.

Cleveland, J.N., Murphy, K.R., Williams, R.E. (1989). Multiple uses of performance appraisal: Prevalence and correlates. Journal of Applied Psychology, 74, 130-135.

Cleveland, J., \& Shore, L. (1992). Self- and supervisory perspectives on age and work attitudes and performance. Journal of Applied Psychology, 77, 469-484.

Darling-Hammond, L., \& Snyder, J. (2000). Authentic assessment of teaching in context. Teaching \& Teacher Education, 16, 523-545.

Dochy, F., \& McDowell, L. (1997). Assessment as a tool for learning. Studies in Educational Evaluation, 23, 279-298.

Evans, A., Ali, S., Singleton, C., Nolan, P., \& Bahrami, J. (2002). The effectiveness of personal education plans in CPD: An evaluation. Medical Teacher, 24 (1), 79-84.

Fenwick, T.J. (2006). Contradictions in portfolio careers: work design and client relations. CareerDevelopment International, 11, 66-79.

Gelderman, C.J., \& van Weele, A.J. (2002). Strategic direction through purchasing portfolio management: A case study. The Journal of Supply Chain Management, 38 (2), 30-37.

Gibbs, G. (1999). Using assessment strategically to change the way students learn, In S. Brown \& A. Glasner (eds.), Assessment Matters in Higher Education. Buckingham: Society for Research into Higher Education and Open University Press.

GITP (2008). HR(D) in Limburgs perspectief. [HRD in Limburg, the Netherlands]

Hattie, J., \& Timperley, H. (2007). The power of feedback. Review of Educational Research, 77, 81-112.

James, M., \& Pedder, D. (2006). Beyond method: Assessment and learning practices and values. The Curriculum Journal, 17, 109-138.

Leggett, M., \& Bunker, A. (2006). Teaching portfolios and university culture. Journal of Further and Higher Education, 30, 269-282.

Lam, S.S.K., \& Schaubroeck, J. (1999). Total quality management and performance appraisal: an experimental study of process versus results and group versus individual approaches. Journal of Organizational Behavior, 20, 445-457.

Lepak, D.P., \& Snell, S.A. (1999). The human resource architecture: Toward a theory of human capital allocation and development. The Academy of Management Review, 24, 31-48. 
Lin, C., Tan, B., \& Hsieh, P. (2005). Application of the Fuzzy Weighted Average in Strategic Portfolio Management. Decision Sciences, 36, 489-511.

London, M. (1997). Job feedback: Giving, seeking, and using feedback for performance improvement. Mahwah, NJ: Erlbaum.

Martins, E.C., \& Terblanche, F. (2003). Building organisational culture that stimulates creativity and innovation. European Journal of Innovation Management, 6, 64-74.

McDowall, A., \& Fletcher, C. (2004). Employee development: An organizational justice perspective. Personnel Review, 33, 8-29.

McMullan, M., Endacott, R., Gray, M.A., Jasper, M., Miller, C.M.L., Scholes, J., \& Webb, C. (2003). Portfolios and assessment of competence: A review of the literature. Journal of Advanced Nursing, 41, 283-294.

Nathan, B.R., Mohrmann, A.M., \& Milliman, J. (1991). Interpersonal relations as a context for the effects of appraisal interviews on performance and satisfaction: A longitudinal study. Academy of Management Journal, 34, 352-369.

Orland-Barak, L. (2005). Portfolios as evidence of reflective practice: What remains "untold". Educational Research, 47 (1), 25-44.

Perrenoud, P. (1998). From formative evaluation to a controlled regulation of learning processes. Towards a wider conceptual field. Assessment in Education: Principles, Policy \& Practice, 5, 85-102.

Prosser, M., \& Trigwell, K. (1999). Understanding learning and teaching: The experience in higher education. Buckingham: Open University Press.

Read, W.H. (1996). Managing the knowledge-based organization: Five principles every manager can use. Technology analysis and strategic management, 8, 223-232.

Redman, W. (1994). Portfolios for development: A guide for trainers and managers. London: Kogan Page.

Riley-Doucet, C., \& Wilson, S. (1997). A three-step method of self-reflection using reflective journal writing. Journal of Advanced Nursing, 25, 964-968.

Schmitz, C.C., \& Schillo, B.A. (2005). Report Carding: A model for foundation portfolio assessment. American Journal of Evaluation, 26, 518-531.

Seng, S.H., \& Seng, T.O. (1996). Reflective teaching and the portfolio approach in early childhood staff development. Paper presented at the Joint Conference of the Educational Research Association of Singapore and the Australian Association for Research in Education. Singapore, 25-29 November 1996.

Smith, K., \& Tillema, H. (1998). Evaluating portfolio use as a learning tool for professionals. Scandinavian Journal of Educational Research, 42, 193-205.

Smith, K., \& Tillema, H. (2001). Long-term influences of portfolios on professional development. Scandinavian Journal of Educational Research, 45, 183-202.

Smith, K., \& Tillema, H. (2003). Clarifying different types of portfolio use. Assessment \& Evaluation in Higher Education, 28, 625-648.

Snadden, D., Thomas M.L., Griffin, E.M., \& Hudson, H. (1996). Portfolio-based learning and general practice vocational training. Medical Education, 30, 148-152.

Snyder, J., Pippincott, A., \& Bower, D. (1998). The inherent tensions in the multiple use of portfolios in teacher education. Teacher Education Quarterly, 25 (1), 45-60.

Tigelaar, D.E.H., Dolmans, D.H.J.M., Wolfhagen, I.H.A.P., \& van der Vleuten, C.P.M. (2004). Using a conceptual framework and the opinions of portfolio experts to develop a teaching portfolio prototype. Studies in Educational Evaluation, 30, 305-321.

Tigelaar, D.E.H., Dolmans, D.H.J.M., De Grave, W.S., Wolfhagen, I.H.A.P., \& Van der Vleuten, C.P.M. (2006). Portfolio as a tool to stimulate teachers' reflections. Medical Teacher, 28, 277-282.

Tillema, H.H. (2001). Portfolios as Developmental Assessment Tools. International Journal of Training and Development, 5, 126-135. 
Tillema, H. (2003). Auditing assessment practices in organisations: Establishing quality criteria for appraising competencies. International Journal of Human Resources Development and Management, 3, 359-370.

Van de Wiel, M.W.J., Szegedi, K.H.P., \& Weggeman, M.C.D.P. (2004). Professional learning: Deliberate attempts at developing expertise. In: H.P.A. Boshuizen, R. Bromme, H. Gruber (Eds.) Professional Learning: Gaps and transitions on the way from novice to expert (pp. 181-206). Dordrecht, the Netherlands: Kluwer.

Van Gennip, N., Gijbels, D., Segers, M., \& Tillema, H. (2010). Reactions to $360^{\circ}$ feedback: The role of trust and trust-related variables. International Journal of Human Resource Development and Management, 10, 362-379.

Wildy, H., \& Wallace, J. (1998). Professionalism, portfolios and the development of school leaders. School Leadership and Management, 18 (1), 123-140.

Wintermantel, R.E., \& Mattimore, K.L. (1997). In the changing world of human resources: Matching measures to mission. Human Resource Management, 36, 337-342.

Wolf, K., \& Dietz, M. (1998). Teaching portfolios: Purposes and possibilities. Teacher Educational Quarterly, 25 (1), 9-22.

Xiao, J. (1996). The relationship between organizational factors and the transfer of training in the electronics industry in Shenzhen, China. Human Resource Development Quarterly, 7, 55-72. 



\section{CHAPTER 5}

\section{THE PERSONAL DEVELOPMENT PLAN QUESTIONNAIRE (PPQ): THE DEVELOPMENT AND VALIDATION OF AN INSTRUMENT TO ASSESS EMPLOYEES' PERCEPTION OF THE PDP PRACTICE ${ }^{13,14}$}

Confronted with the speed of technological advancements and increasing global competition, organizations have come to realize that their employees' continuous learning drives business success. A popular tool to support and enhance continuous learning is the Personal Development Plan (PDP). Despite its popularity, empirical evidence of the effectiveness of the PDP is scarce. To date, most of the research on the practice of the PDP is conducted within educational (students) and health (general practitioners) settings and not within business environments. Moreover, research methodology is restricted to qualitative methods and survey research is rare. For that reason we developed and validated a questionnaire to assess personal development plan practices. To develop the questionnaire we relied on literature on portfolio assessment in the educational context and the workplace (health and education); to validate it we collected data in three independent organizations. To assess the factor structure of the questionnaire, exploratory principal component factor analyses with direct oblimin rotation were conducted on data sets from two organizations. Factor reliability was computed using Cronbach's alpha coefficient. The principal component analysis resulted in a 35-item questionnaire containing five factors each with high internal validity. Cronbach's alphas ranged between .63 to .91 . Next, a confirmatory factor analysis was conducted on an independent sample of 287 employees, working in a third organization. The results from this analysis were used to further refine the instrument. The present instrument provides a reliable and valid measure of the employee's perception of the personal development plan practice in the workplace, consisting of four scales and 19 items

13 Based upon Beausaert, S., Segers, M., Gijselaers, W. (accepted). The Personal Development Plan Practice Questionnaire (PPQ): The development and validation of an instrument to assess employees' perception of the personal development plan practice. International Journal of Training and Development, 15 (5).

14 We would like to thank Janine van de Rijt, for her detailed corrections. 


\section{Introduction}

Over the course of the last couple of decades organizations have come to realize that their employees' continuous learning drives business success. With that in mind, they consider ways to stimulate their employees' lifelong learning and make sure employees are able to deal with new situations (Boud, 2001; Bolhuis, 2003). Lifelong learning is not only enhanced by organizing off-the-job training programs or courses, but increasingly by stimulating formal and informal learning activities in the workplace (e.g. learning-by-doing, coaching and team learning) (Bartram \& Roe, 2008). Personal Development Plans (PDP), mostly discussed during performance interviews, have become popular tools in structuring and organizing the employee's learning (Austin, Marini, Desroches, 2005).

The origins of the PDP practice can be traced back to the portfolios that were used as a showcase by photographers, painters, architects and brokers for a long time (Lyons \& Evans, 1997; Mathers, Challis, Howe, \& Field, 1999). Students in secondary schools and higher education have been using portfolios too, with the main purpose of supporting learning or to provide a certificate. Student portfolios in most cases consist of a PDP, a collection of pieces of evidence and a reflection report (Driessen, van Tartwijk, Van der Vleuten, \& Wass, 2007). PDPs have recently been increasingly used in organizations as well. Since the PDP promises to be a useful tool in managing the employee's continuing professional development, it spurs a lot of interest (e.g. Firssova, 2006). This is reflected in the vast amount of theoretical and practice-based literature on the subject (e.g. Wilkinson et al., 2002; Wright, Knight, \& Pomerleau, 1999), conferences and international workshops or seminars on portfolio assessment and/or PDP's specifically (for example, The 5th International ePortfolio Conference 2007, 27-29 September 2007).

While past research focuses on the use of portfolio assessment by students (e.g. Driessen et al., 2007) relatively little is known about the use of the PDP as such and its impact on the employee's learning and development. Empirical evidence on the use and effectiveness of PDPs in organizations is limited (Austin et al., 2005; Evans, Ali, Singleton, Nolan, \& Bahrami, 2002; Zeichner \& Wray, 2001). Furthermore, the small amount of empirical research that does focus on the use of PDPs in organizations, mostly researches it in an educational (teachers) or health setting. It is assumed that the relatively high amount of research on the subject in the educational field and health context compared to that in a business setting is due to the fact that, as is pointed out by Mathers et al. (1999), the instrument has been used in those contexts for much longer than in business contexts.

In order to contribute to the research on the use and effectiveness of PDPs, this article provides a research-based solution for diagnosing the PDP practice in organizations. It provides managers with an instrument to evaluate the PDP practice and Human Resource Development in their organization. This instrument 
screens and evaluates the conditions which support the PDP practice and the Human Resource Development process, and helps determine which supporting conditions, i.e. the role of the supervisor and the way feedback is delivered, need to be improved. The instrument validated in this study can be used for such a general screening of the human resource development in organizations. First, we present the conceptual basis for the construct called 'PDP practice'. Second, the theoretical basis for the PDP practice questionnaire is described. Third, the systematic development and validation of the instrument is explained, which will help managers in organizations to gain insight in and optimize the PDP practice.

\subsection{A Personal Development Plan: Toward a definition}

Definition. Personal Development Plans (PDP) take various forms and are referred to by different synonyms: portfolio, (continuing professional) development plan, logbook or personal professional profile. The two most commonly used concepts are (1) professional development plan, and (2) portfolio assessment. From this point on in this study we will only use the term PDP, however, to avoid all confusion. The term portfolio assessment carries many different connotations separate from the one we mean here in organizational literature. Portfolio (assessment) can refer to a report system that is used for organizational accountability and learning (e.g. Schmitz \& Schillo, 2005), for example, or to portfolio management, aiming at the development and implementation of purchasing strategies (e.g. Gelderman, \& van Weele, 2002; Lin, Tan, \& Hsieh, 2005). Another connotation is that of portfolio work, a form of flexible self-employment in which individuals contract their skills and knowledge to different persons and organizations and develop a portfolio of job activities for themselves (Fenwick, 2006).

The different synonyms mentioned above for what we will from here on refer to as the PDP are often used to indicate instruments with different characteristics and ranges of application. In spite of the differences, those instruments have several characteristics in common ${ }^{15}$. A recent literature review by Beausaert, Segers, Van der Rijt and Gijselaers (2011) identified four characteristics to describe the assessment tool, used in organizational settings:

- gives an overview of the competencies the employee worked on in the past and which competencies the employee is planning to work on in the future and how;

- $\quad$ should be composed by the employee himself, mostly in consultation with the supervisor;

15 For clarity reasons we will use the term 'personal development plan' or 'PDP' in this chapter, even if the literature source we are referring to uses one of the synonyms. 
- $\quad$ can be used as a basis/structure for conversations with the supervisor or coach who provides the employee with feedback and stimulates the employee's reflection; and

- $\quad$ serves as a decision-making tool, from planning an individual training-program to assessing the suitability of a promotion.

Goal. In the PDP, three major questions are put forward: 'Where am I going?', 'How am I going?', and 'Which step to take next?'. When a discrepancy is detected between the competencies an employee possesses and the competencies the employee should possess, it suggests that learning needs to be stimulated (Hattie \& Timperley, 2007). This means that the core purpose of the PDP is supporting employees' development in order to improve performance. With respect to the purpose of a PDP, a difference has commonly been made between professional development and certification/selection/accountability (Smith \& Tillema, 2001). When a personal development plan is used for development purposes, learning takes a central part. Conversely, when a PDP is used for certification, presenting oneself is more important. Additionally, a literature review on the goals of PDPs used in the workplace identified nine clusters of goals (Beausaert et al., 2011), namely:
(1) professional development;
(2) reflective learning;
(3) providing evidence;
(4) documenting;
(5) certification, selection and promotion;
(6) external mobility;
(7) coaching;
(8) stimulating confidence; and
(9) organizing.

Moreover, additional to the distinction made between different goals, Smith and Tillema (2001) make a difference between composing a PDP on a voluntary basis and on a mandatory basis. The results of the Smith and Tillema study (2001) indicated that employees who voluntarily use a personal development plan show more professional development than those who are mandated to do so. In contrast, other research showed that staff members did not develop a [teaching] PDP unless they were obliged to (mandatory) (Bunker \& Leggett, 2004).

Different types of PDPs. In line with the distinctions between the different goals, distinctions have been drawn between several types of portfolios. A working portfolio is used for reflection, while a documentary portfolio and a show portfolio are used for summative assessment. The difference between a documentary portfolio and a show portfolio is that a show portfolio only displays the best of someone's 
work (Bunker \& Leggett, 2004). Similarly, Smith and Tillema (2003) distinguish four types of portfolio use, which can be described as follows:

- The dossier portfolio: mainly for selection purposes, required for entry into a profession or program; a record of achievement or a collection of work (e.g. a photographer's portfolio).

- The training portfolio: a fixed format which helps the person to collect evidence of acquired competencies during a training program. It contains a representative sample of the person's work.

- $\quad$ The reflective portfolio: like the dossier portfolio also used for promotion and selection purposes, but on a voluntary basis. It is personally collected evidence of growth and accomplishments and it reveals best practices. Self-appraisal is important.

- The personal development portfolio: evidence of professional growth during a long-term process. It is voluntary and compiled for learning and development purposes. It stimulates the conversation with colleagues and, consequently, the reflection on experiences and the refinement of one's growth.

In sum, a PDP, as defined above, can be used for different purposes, resulting in different types of PDPs. The tool is mostly used to structure and stimulate the learning and professional development of the employee. In order to accelerate the employee's learning, attention should be paid to several supporting process conditions (Turner, Mavin, \& Minocha, 2006).

\subsection{Supporting process conditions}

The literature on PDP assessment in the workplace indicates that in order for the PDP assessment process in organizations to work smoothly and successfully, several supporting process conditions need to be met, inherent to how we defined a PDP (Beausaert et al., 2011). A distinction is drawn between supporting process conditions related to the implemented assessment process (contextual supporting process conditions) and conditions related to the user of the individual development plan (individual supporting process conditions).

\subsubsection{Contextual supporting process conditions}

The power of a PDP lies in the support the tool provides to professional development, more than the support it provides for certification or selection purposes (Tillema, 2001). In order to stimulate the employee's professional development, the presence of a supervisor that provides sufficient constructive feedback is crucial (Ashford, 1986; Ilgen, Fisher, \& Taylor, 1979; Kluger \& DeNisi, 1996). Those conditions, of supervisor support and feedback, are widely thought to be essential in order to make a PDP work. In other words, the implementation of a PDP does not guarantee that it will stimulate professional development. It has to be embed- 
ded in a learning climate where supervisor support and feedback are part of the equation.

\section{The motivating supervisor}

Literature argues that using a PDP needs to be accompanied by sufficient support (London \& Smither, 1999). For example, researchers found that dentists who received support from a supervisor [tutor] undertook more learning activities (Bullock, Firmstone, Frame, \& Bedward, 2007). Similarly, it was found that employees are more likely to engage in developmental activities such as training when they have supervisors that are supportive of their efforts (London et al., 1999). Employees need to be supported in a non-controlling way that empowers selfdevelopment, and should be provided with behavioral choices for learning. This in turn will lead to increased intrinsic motivation, as employees experience a sense of self-competence and feel they are controlling their own behavior (London, Larsen, \& Thisted, 1999; London \& Smither, 1999). Support can be offered by a coach, a mentor or a supervisor. In organizations, the assessment process in which a PDP is used is mostly supported by the supervisor. This is consistent with earlier findings revealing that in using a dossier, training and reflective PDP, external feedback was most valued when it was given by a superior (Smith \& Tillema, 2003). A supervisor should be readily available and have sufficient contact with the employee (Wasylyshyn, 2003). The lack of interaction with the supervisor is mostly due to time limitations, incompatible work schedules, and physical distance (Noe, 1988). Additionally, the effects of personal development plans were found to be more profound if supervisors provide their employees with a clear goal, a formal framework (including meetings), specific guidelines and standards (e.g. Guaglianone, 1995; Noe, 1996; Roberts, 2003).

\section{Feedback}

Feedback given by a supervisor, coach or mentor to the employee within the context of using PDPs, is a form of communication conveying a message to the employee which contains information about him or her (Ashford, 1986) for the purpose of improving learning and performance (Shute, 2008). That feedback plays a crucial role in making the PDP assessment process effective (e.g. Tigelaar, Dolmans, De Grave, Wolfhagen, \& Van der Vleuten, 2006). However, in a meta-analysis on the effects of feedback interventions on performance, Kluger and DeNisi (1996) found no consistent pattern of results. They concluded that the effects of feedback are not always positive and need to be researched more deeply.

Feedback quality. Employees need to be provided with nonthreatening performance feedback (London \& Smither, 1999). The feedback should focus on employees' attention to the task, giving constructive suggestions on what could be changed to improve employee effort. Moreover, feedback should focus on em- 
ployees' performance behavior and learning and not on the employee as a person or the performance compared with colleagues (Tillema \& Smith, 2000). By focusing on the individual or comparing the employee with his colleagues, employees' motivation and self-efficacy are undermined, while self-efficacy is important to employees' effort and task persistence (Gibbs \& Simpson, 2004).

Kluger and DeNisi (1996) developed the Feedback Intervention Theory (FIT) and made a distinction between three groups of goals on which feedback interventions can focus, namely Meta-task processes (self), Task-motivation processes (focal task) and Task-learning processes (task details). Research has shown that feedback interventions usually focus on task-motivation processes. The interventions shift attention away from the individual as a person and the specific details of the task, which can be performed without much conscious effort. In order for the feedback to be seen as acceptable and valuable by the employee, the supervisor has to be perceived as credible (Ilgen et al., 1979). Furthermore, the quality of feedback is enhanced by focusing on a goal and how to attain that goal (Gibbs \& Simpson, 2004). If employees are able to link the feedback to their performance and the supervisor's expectations of their performance, the feedback becomes meaningful (Ilgen et al., 1979). Feedback should target specific behavior and be clear. Specific feedback not only indicates what is right or wrong; it also indicates ways to improve. In contrast, feedback that is not specific tends to impede learning. It induces uncertainty in responding to feedback and requires greater cognitive processing to understand the feedback. Both inhibit the employee's motivation. As a consequence, the feedback is perceived as useless and it evokes frustration (London et al., 1999; London \& Smither, 1999; Shute, 2008). Finally, it may be ineffective to simply provide corrective feedback and it leads to resistance when given too frequently (Butler \& Winne, 1995).Corrective, negative or extensive feedback seems to be accepted and followed less easily than positive feedback (Yammarino \& Atwater, 1993).

Feedback quantity and timing. While feedback should provide enough details and be clear, the feedback should not be too extensive or too complicated because this kind of feedback loses impact after a while (Shute, 2008). It was found that too much feedback on a task inhibits employees' feeling of control on the job task. Both feelings of control and feelings of competence must be high for employees to be intrinsically motivated (Ilgen et al., 1979). In contrast, a lack of feedback could lead to anxiety, inaccurate self-evaluations, and a diversion of effort towards feedback gathering activities (e.g. performance interviews) (Taylor, Fisher, \& Ilgen, 1984). Lastly, research on the relationship between feedback timing and learning and performance found different results. While many field studies explained the positive effects that immediate feedback seemed to have, laboratory studies showed the positive results of delayed feedback (Shute, 2008). 
Feedback use. Feedback use refers to the employee's response to the feedback. This response is essential for effective PDP assessment, since feedback needs to be received and attended to by employees in order to contribute to development and performance (Gibbs, Simpson, \& MacDonald, 2003; Gibbs \& Simpson, 2004; Tillema, 1998). Furthermore, studies showed that feedback influences performance positively under certain conditions, depending on the characteristics of the feedback (source, message, amount and frequency) (Alder, 2007; Larson, 1989).

\subsubsection{Individual supporting process conditions}

Austin et al. (2005, p. 181) speaks of the PDP as an instrument that requires "an idealized type of individual who knows how to self-reflect, is open to change, interested in his own development and knows how to organize her/himself and her/his environment to support learning". Below we will elaborate on the employee's ability to reflect, a competence an employee needs to possess in order to learn from using a PDP; and on the amount and the distribution of the employee's effort.

\section{Reflection}

Reflection refers to a critical analysis of previous experiences that aims to intensify cognitive elaboration on those experiences and leads to behavioral changes (Anseel, Lievens, \& Schollaert, 2009; McMullan et al., 2003; Seng \& Seng, 1996; Smith \& Tillema, 1998). In other words, reflecting provides insight into the employee's own process of learning (McMullan et al., 2003). That process of individual learning can best be explained by the experiential learning cycle of Kolb, in which reflection on personal experiences plays a central role (Kolb, 1984). According to this theory learning occurs through 'concrete experiences'. Afterwards the employee reflects on what he experienced, potentially by using a PDP. By thinking over what happened, the learner is able to draw more general conclusions (abstraction) and build up concepts (conceptualization). In a fourth step the learner can use those previous experiences and what $s /$ he learned from them as a basis for new active experimentation and improve her/his performance.

\section{Amount and distribution of employee effort}

The amount and distribution of employee effort is the amount and distribution of time spent working on the PDP. The assessment should take sufficient study time and effort and these should be evenly distributed over time (Chickering \& Gamson, 1991; Gibbs \& Simpson, 2004). Research suggests a direct relationship between the quantity and quality of employee effort to learn and develop (Berliner, 1984; Gibbs, Simpson, \& MacDonald, 2003; Gibbs \& Simpson, 2004).

Within the broader perspective of using a PDP to stimulate employees' continuous professional development, an even distribution of time and effort is impor- 
tant. A study focusing on the use of the PDP as a tool for providing functional feedback, Smith and Tillema (1998) concluded that high quality PDPs can only be reached after sustained use. Sustained use could depend on the functional feedback it delivers, and on the perceived usefulness for continuing professional and personal development. In contrast, employees do not maintain a PDP because it is time-consuming, not mandatory or not helpful in short-term professional development (Smith \& Tillema, 2001).

In conclusion, it is these supporting conditions, i.e. the motivating supervisor, the quantity, quality and use of the feedback given by the supervisor and the employee's effort and learning, that the questionnaire we developed and validated will help screen and evaluate.

\section{Questionnaire development: Constructs and measures}

We developed and validated the Personal Development Plan Practice Questionnaire (PPQ) to measure the employee's perception of the personal development plan practice by questioning the supporting process conditions. To our knowledge, this is the first instrument approaching the evaluation of the PDP practice in that way. Most existing portfolio assessment questionnaires are specifically tailored for use in a formal schooling context (students who deliver their portfolio as part of the exam requirements); the purpose of the current study is to develop and validate an instrument that can be used to assess the practice of personal development plans in organizations. To measure the effectiveness of the PDP assessment practice, it is important to gain insight into employees' perception of that practice. Research has shown that employees' perception of the assessment practice influences their eventual learning outcomes (Biggs, 2003; Prosser \& Trigwell, 1999). After all, the 3P model for students' learning of Biggs (2003) and Prosser and Trigwell (1999), which originally discusses the assessment practice as a tool in student learning but is easily adaptable to an organizational setting, shows that the assessment practice influences the student's learning outcomes through the student's perception of that practice.

In developing the questionnaire we drew inspiration from the Assessment Experience Questionnaire (AEQ) and its theoretical basis (Gibbs \& Simpson, 2004). Although this questionnaire measures assessment in an educational context (students), it can be related to the PDP practice in organizations. It is one of the few questionnaires that measure supporting conditions for stimulating learning, in which lies the power of the PDP. Furthermore the questionnaire had already been adapted for portfolio assessment once before, although again for portfolio assessment as used by students (Segers, Gijbels, \& Thurlings, 2008). The questionnaire 
contains 36 items categorized in six five-point Likert scales: amount and distribution of employee effort ( 6 items, e.g. "It is possible to do quite well without studying too much for this training"), the PDP assessment and learning (6 items, e.g. "Filling in the PDP brought things together for me"), quantity and timing of feedback ( 6 items, e.g. "The feedback comes back very quickly"), quality of feedback ( 6 items, e.g. "The feedback helps me to do things better"), what you do with the feedback (use/effect) ( 6 items, e.g. "I tend to only read the end evaluation") and the evaluation (in this case, Constructing the PDP; 6 items, e.g. "To construct the PDP I only summed up some facts"). We found further inspiration in the Feedback Environment Scale (FES; Steelman, Levy, \& Snell, 2004). The FES measures different aspects of the feedback environment in which managers and co-workers play a central role, namely: source credibility (5 items, e.g. "My supervisor is generally familiar with my performance on the job."), feedback quality (5 items, e.g. "My supervisor gives me useful feedback about my job performance."), feedback delivery ( 5 items, e.g. "My supervisor is supportive when giving me feedback about my job performance."), favorable and unfavorable feedback (4 items +4 items, e.g. "When I do a good job at work, my supervisor praises my performance."), source availability ( 5 items, e.g. "My supervisor is usually available when I want performance information.") and the promotion of feedback-seeking behavior (5 items, e.g. "My supervisor is often annoyed when I directly ask for performance feedback.").

Because an employee needs to be supported in a non-controlling way that empowers self-development and should be provided with behavioral choices for learning (London et al., 1999; London \& Smither, 1999), we developed and added a scale to measure the way the supervisor supports the assessment practice and motivates the employee ( 5 items, e.g. "My manager can be seen as involved") (London \& Smither, 1999).

Based on a pilot study in two different service departments of Maastricht University ( $\mathrm{N}=27$ and $\mathrm{N}=15$ ); and consulting with HRM-managers from different organizations $(N=12)$, we adapted the questions of the AEQ that were used in an educational context to the organizational setting. For example, feedback is often given during performance interviews and not on products gathered in the tool, which is what students used to do when constructing a portfolio, which resulted in the adaptation and reformulation of the feedback questions. Next, because employees do not undertake a summative test like students do, the focus in the evaluation scale was on constructing the personal development plan. Consequently, the scale was no longer linked to getting a grade or a judgment as primary goal. The managers also suggested to not only research the PDP as a separate tool, but to link it with the performance assessment cycle, consisting of performance, continuation and judgment interviews in which the PDPs are discussed. Figure 1 and Table 1 
show the different personal development plan practice scales incorporated in the questionnaire.

\section{Figure 1}

Conceptual model

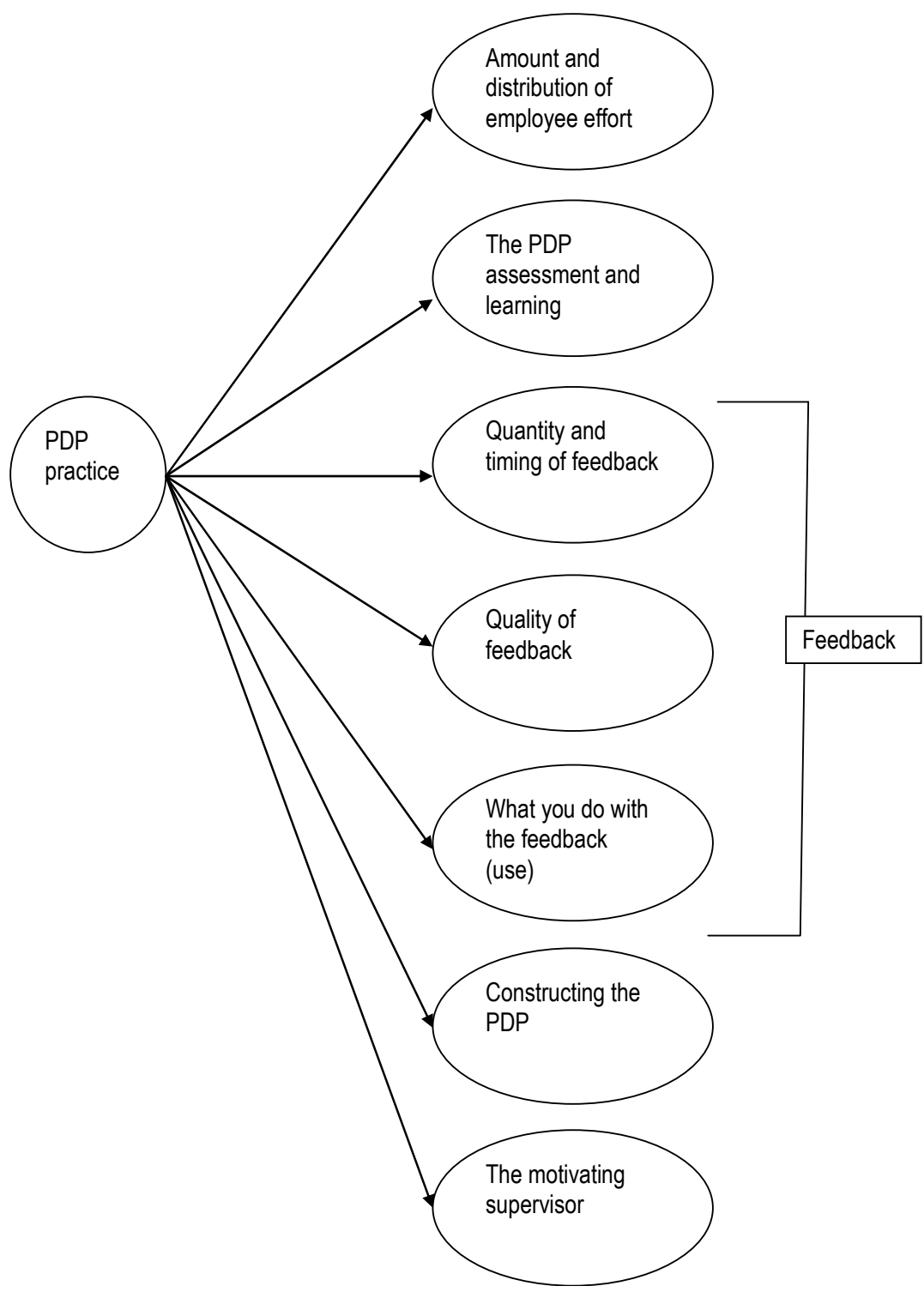


Table 1

Overview of the constructs, variables and instruments used

\begin{tabular}{ll}
\hline Construct & Variable \\
\hline The PDP practice & 1. Amount and distribution of employee effort \\
2. The PDP assessment and learning \\
3. Quantity and timing of feedback \\
4. Quality of feedback \\
5. What you do with the feedback (effect) \\
6. Evaluation (constructing the PDP) \\
7. The motivating supervisor
\end{tabular}

\section{Method}

In order to validate the newly developed questionnaire, first an exploratory factor analysis and next a confirmatory factor analysis were conducted. The exploratory factor analysis was performed on the data collected in Organizations 1 and 2, while the confirmatory factor analysis was performed on the data collected in Organization 3. The latter was done to optimise the observed and theoretical factor structures for the data collected in Organizations 1 and 2. In other words, the "goodness of fit" of the predetermined factor model was determined across different organizational settings (Stapleton, 1997), what renders the instrument useful within more and more diverse organizations. Below we will discuss the different groups of participants, the instrument and the data analysis in more detail.

\subsection{Sample: Contexts and participants}

The participants are employees, working in three different organizations.

Organization 1. The participants are employees of an international organization specialized in global business services and who compile and use a personal development plan. The organization employs approximately 330.000 people across 220 countries. The offices that participated in the study employ 400 people in total. The organization refers to the tool as an 'Individual Development Plan' (IDP) and implemented it approximately three years ago as part of an assessment cycle. The assessment cycle takes one year; the employee has a performance interview, an assessment interview and a career interview with her/his supervisor. On top of those more formal interviews, the employee has five to six informal meetings with her/his supervisor in which the IDP can be discussed.

400 employees were contacted; out of these 400 a final total of 103 (40 men and 60 women; 3 missing values) participated in the research, spread over 12 different departments, yielding a response rate of $26 \%$. Of the 100 employees who provided us with their background characteristics, 24 had an academic master 
degree, 49 had a professional bachelor's degree, 25 had a degree secondary education and two had a degree elementary education. The average employee was between 36 and 40 years old (half aged between 25 and 36 years). The average number of years of experience was six to eight years. 13 of the participants had recently achieved a promotion.

Organization 2. The participants are experts from an international organization that specializes in medical technology. The total organization employs approximately 38.000 people in 120 countries. The office that participated employs 200 people in total. The assessment cycle at this organization is similar to the one in Organization 1. First, over the course of one year, the personal objectives of the employee are determined. Next, the employee has to fill out a performance measure and a PDP; a selected group of employees also works on a talent portfolio. The three instruments are linked to each other and all of them are discussed during a meeting. We treat the three instruments as one. Furthermore, evaluating the effectiveness of the PDP, we do not only refer to the tool, but also to the meetings in which the PDP is discussed.

Out of the 200 employees that were contacted, a final total of 84 (30 men and 50 women; 4 missing values) participated in the research, a response rate of $42 \%$. Seven had a secondary education degree, 21 had a professional bachelor's degree and 52 had an academic master degree or a PhD. The average age was between 35 and 41 . The number of years of experience in the current role was on average between five and 10 years.

Organization 3. The participants are employees of a regional Dutch governmental office. 1400 employees work in five different offices, located in four different cities. Despite the relatively large amount of employees working in different departments, the organization is perceived as a mid-sized organization. Once they have entered the organization, most employees stay put. The organization is considered to be a warm working environment. As a result, the average age of the employees was 49 in 2009. Organization 3 has a very low turn-around of employees.

Employees of the governmental office annually undergo an assessment cycle. The assessment cycle consists of a performance interview, a development interview and an assessment interview with the supervisor. During the assessment process the supervisor and the employee can make use of two different supporting instruments, namely the 'Knowledge-ID' and the 'Evaluation-form'. While the Knowledge-ID provides the supervisor with an extended CV of the employee's previous learning experiences, the Evaluation-form not only looks backward, but also determines the competencies an employee still needs to develop. Recently, the central HR-office of the governmental offices in the Netherlands decided to implement a new instrument, namely a Personal Development Plan (PDP). The PDP inte- 
grates the Knowledge-ID and the Evaluation-form by listing the competencies the employee still needs to develop (looking forward) through evaluating her/his strengths and weaknesses (looking back). The tools are not used as strictly by every supervisor, however. When we use the term Personal Development Plan in regard to Organization 3 in this article, we refer to all three, meaning the Knowledge-ID, the Evaluation-form and/or the PDP.

Out of the 1400 employees contacted, a total of 287 (response rate 21\%, 187 men and 72 female; 28 missing values) participated in the research, spread over at least six different departments and four office locations. Of the 259 employees who provided us with their highest education level, 27 studied WO (university), 81 HBO (non-academic higher education), 16 VWO (academically-oriented secondary education), 47 HAVO (higher secondary education), 55 MBO (higher secondary vocational education) and eight VMBO (lower secondary vocational education). The average employee was 49 years old $(S D=7)$. The average number of years of experience in the organization was 21 years $(S D=12)$ and the average number of years of experience in the current role was 10 years $(S D=9)$. These numbers are in line with the statistics that are available for the total group of employees.

\subsection{Procedure}

The organizations were contacted and invited to participate in the research. After a positive introductory meeting with managers of the HRM-department, the questionnaire was adapted to the specific setting of the organization in cooperation with this department. Lastly, the questionnaires were distributed by the HRdepartment via email, with a link to the online questionnaire. To guarantee the anonymity of the employees, the data were gathered immediately by the software (NetQ). To increase the response rate, the employees received two reminders.

\subsection{The instrument: Measures}

The questionnaire contains three sections. The first section collects demographic data (gender, age, education, department, function) and the second section collects data about the implementation of the instrument. Neither of the sections was included in the statistical analyses. Section 3 specifically questions the personal development plan practice and use and consists of 47 items, distributed among seven scales: Amount and distribution of employee effort, The PDP assessment and learning, Quantity and timing of feedback, Quality of feedback, What you do with the feedback, Constructing the PDP and The motivating supervisor. All questions had to be answered making use of a 5-point Likert scale, ranging from totally agree to totally disagree. 


\subsection{Data analysis}

The questionnaire was validated in two steps: (1) exploratory analysis, to explore the optimal factorial structure of the questionnaire; (2) confirmatory analysis, to determine the robustness of the factor structure across samples. For the exploratory factor analysis, the data collected in Organizations 1 and 2 were used. For the confirmatory analysis, the data collected in Organization 3 was used.

\subsubsection{Exploratory factor analysis ${ }^{16}$}

To explore the structure components of the items and select the strongest items for confirmatory analyses, a Principal Component Analysis (PCA) was used, with SPSS version 15.0 (Gerbing \& Hamilton, 1996). As a starting point in the selection of the number of components, an analysis of the eigenvalue decomposition ( $>1$ criterion) was made and the screeplot was inspected (Fabrigar, Wegener, MacCallum, \& Strahan, 1999). Next, in case more than seven components were retained, a direct oblimin rotation ${ }^{17}$ was conducted with a pre-specified number of components, depending on the principal component analysis.

\subsubsection{Confirmatory factor analysis}

By performing a structural equation modelling in EQS version 6.1, the stability of the proposed factor structure was tested. The following rules were used to interpret the fit of the model: the Root Mean Square Error of Approximation (RMSEA) values should be as small as possible, with perfect fit indicated by an index of zero and values less than 0.05 indicating a good fit (Browne \& Cudeck, 1992). Furthermore, the standardized root mean squared residual (SRMR; Bentler, 1990) should be smaller than 0.08. Finally, a good model fit is indicated by a CFI score above 0.90 (Byrne, 1998). Byrne (1998) indicated that the $\chi^{2}$ statistic becomes increasingly unreliable in sample sizes above 250 participants. Therefore, a slightly less sampledependent statistic was used: $\chi^{2}$ divided by its degree of freedom. Any number below 5 indicates a good model fit (Bollen, 1989).

16 Factor analysis (and principal component analysis) is "a technique for indentifying groups or clusters of variables. This technique has three main uses: (1) to understand the structure of a set of variables; (2) to construct a questionnaire to measure an underlying variable; and (3) to reduce a data set to a more manageable size while retaining as much of the original information as possible." (Field, 2005).

17 When using direct oblimin rotation factors are allowed to correlate. "The degree to which factors are allowed to correlate is determined by the value of a constant called delta. The default value in SPSS is zero and this ensures that high correlation between factors is not allowed." (Field, 2005). 


\section{Results}

\subsection{Exploratory factor analysis}

To assess the validity of the scales mentioned in Table 1, we performed a Principal Component Analysis (Table 2) on the items of the seven scales related to the PDP practice and use. Without specifying how many factors to retain, an initial analysis was run and indicated 12 factors with eigenvalues greater than 1 . However, the eigenvalues, the screeplot and a visual inspection of several trial solutions indicated that five factors had to be extracted. Furthermore, the 5-factor model showed the best interpretable factor pattern. The first five components had an eigenvalue from 11.93 to 1.89 , accounting for $47.94 \%$ of the explained variance. The sixth component had an eigenvalue of 1.75 and explained only $3.72 \%$ of the variance; the screeplot also showed almost no curve drop after the fifth factor. Ten items loaded on Factor 1 , accounting for $25.38 \%$ of the variance and were named the Learning and Reflection scale. Factor 2 consisted of 11 items accounting for $7.68 \%$ of the variance; these were labelled the Instruction and Feedback scale. The third factor, on which six items were loaded, explained $5.97 \%$ of the variance and was named the Motivating Supervisor scale. Five items loaded on factor 4, explaining $4.90 \%$ of the variance and were named the Evaluative Nature of the PDP scale. Five items were loaded on the last factor. Factor 5 explained $4.01 \%$ of the variance and was labelled the Effectiveness of the PDP scale.

Three items ("I would learn more if I received more feedback", "The feedback does not help me to develop my PDP further" and "I learn more from making a PDP than from following training") showed a significant cross-load and seven items had structure coefficients lower than .40. Those factors were removed ("To construct the PDP I only summed up some facts"; "I can work very selectively and still have a positive PDP-meeting with my supervisor"; "The feedback helps me to understand things better"; "The feedback indicates how I can do better next time"; "As soon as I handed in my PDP I forget again everything I have learned by making use of the PDP"; "Mostly I only pay attention to the evaluation that is given to me"; "My PDP can be positively evaluated, even if I did not work on my competencies"). 
Table 2

Exploratory factor analysis PDP practice: Pattern matrix $^{18}$

\begin{tabular}{|c|c|c|c|c|c|}
\hline Item & $\begin{array}{l}\text { Factor } \\
1\end{array}$ & $\begin{array}{l}\text { Factor } \\
2\end{array}$ & $\begin{array}{l}\text { Factor } \\
3\end{array}$ & $\begin{array}{l}\text { Factor } \\
4\end{array}$ & $\begin{array}{l}\text { Factor } \\
5\end{array}$ \\
\hline I learn from the PDP. & .714 & & & & \\
\hline Making the PDP really set me thinking. & .685 & & & & \\
\hline I think the PDP is interesting. & .606 & & & & \\
\hline $\begin{array}{l}\text { The PDP stimulates me to discuss matters with my } \\
\text { supervisor. }\end{array}$ & .581 & & & & \\
\hline $\begin{array}{l}\text { Not working on my competencies has an influence on } \\
\text { my PDP-meeting. }\end{array}$ & .553 & & & & \\
\hline $\begin{array}{l}\text { The PDP stimulates me to discuss matters with my } \\
\text { colleagues. }\end{array}$ & .536 & & & & \\
\hline $\begin{array}{l}\text { When the PDP-meeting is approaching, I spend more } \\
\text { hours on my PDP. }\end{array}$ & .476 & & & & \\
\hline I do not use the feedback to improve something. & -.452 & & & & \\
\hline $\begin{array}{l}\text { With the feedback given, I take another close look at } \\
\text { my PDP. }\end{array}$ & .442 & & & & \\
\hline $\begin{array}{l}\text { I know to what attention is paid when evaluating the } \\
\text { PDP. }\end{array}$ & .422 & & & & \\
\hline $\begin{array}{l}\text { No matter what kind of feedback I am receiving, I } \\
\text { receive it too late to be useful feedback. }\end{array}$ & & .614 & & & \\
\hline $\begin{array}{l}\text { When I'm working on my PDP it is not clear what is } \\
\text { good. }\end{array}$ & & 612 & & & \\
\hline Often the feedback is given too quickly. & & .605 & & & \\
\hline The PDP-form nearly contains no feedback. & & .603 & & & \\
\hline $\begin{array}{l}\text { I can not derive from the feedback what I should do } \\
\text { to improve something. }\end{array}$ & & .603 & & & \\
\hline $\begin{array}{l}\text { If I make a mistake or do not understand something, I } \\
\text { receive not much support to make sure do it well or } \\
\text { understand it. }\end{array}$ & & .574 & & & \\
\hline I receive enough feedback on my PDP. & & -.543 & & & \\
\hline I do not understand everything of the feedback given. & & .542 & & & \\
\hline What you have to do for the PDP is clearly indicated. & & -.500 & & & \\
\hline I spend time on my PDP on a regular basis. & & -.441 & & & \\
\hline $\begin{array}{l}\text { It is clear for me what I should know and be able to } \\
\text { do for the PDP. }\end{array}$ & & -.400 & & & \\
\hline $\begin{array}{l}\text { My supervisor leaves me enough space to determine } \\
\text { the content of the PDP and the related meetings. }\end{array}$ & & & .868 & & \\
\hline My supervisor shows understanding for my situation. & & & .851 & & \\
\hline $\begin{array}{l}\text { My supervisor leaves me enough choice concerning } \\
\text { the content of the PDP and the related meetings (e.g. } \\
\text { 'Which learning activities am I going to undertake?). }\end{array}$ & & & .821 & & \\
\hline My supervisor can be seen as empathic. & & & .814 & & \\
\hline $\begin{array}{l}\text { My supervisor has realistic and do-able expectations } \\
\text { about my PDP. }\end{array}$ & & & .745 & & \\
\hline While developing the PDP, things fell into place. & & & .416 & & \\
\hline
\end{tabular}

18 Pattern matrix: Direct oblimin rotation; 5 factors extracted; maximum iterations for convergence: 25 and absolute values less than .40 suppressed. 
I only cover what should be incorporated in the PDP.

691

.576

The feedback usually indicates how good I am doing,

in relation to my colleagues.

When I have heard/read the feedback, I understand

why I received a certain grade or evaluation.

I have to work on my PDP regularly to get good

evaluations.

If I received feedback, I take another close look at the

competencies we discussed earlier.

Without working a lot on your PDP, you can still have

a positive PDP-meeting.

I read the feedback closely and I try to understand

what the feedback means.

While developing the PDP I learned new things.

After developing a PDP I understand things better.

The PDP is not really challenging.

The alpha coefficients, resulting from the reliability analysis ranged from .63 to .91. Table 3 shows the alpha coefficients for the scales and descriptive statistics. Based on this table, several revisions were made.

The scale Learning and Reflection consisted of three recoded items. However, the internal consistency analysis showed that the items "Not working on my competencies has an influence on my PDP-meeting" and "When the PDP-meeting is approaching, I spend more hours on my PDP" were positively interpreted from an organizational perspective, in contrast to the students' perspective, which meant that the items were not recoded. The scale showed a Cronbach's alpha of .84. The Motivating Supervisor scale, consisting of six items, showed a Cronbach's alpha of .88. All items, except for "While developing the PDP, things fell into place" were related to the supervisor. Because of the different meaning of the latter item in comparison with the other items loading on the factor, this item was excluded. As a consequence, the Cronbach's alpha rose from .88 to .91. The scale the Evaluative Nature of the PDP consisted of one item that was again positively interpreted while it was meant to be recoded, namely the item "I only cover what should be incorporated in the PDP". The Cronbach's alpha was .63. In the last scale, Perceived Effectiveness of the PDP, the poorly performing item "Without working a lot on your PDP, you can still have a positive PDP-meeting" was deleted. As a result, the Cronbach's alpha rose from .52 to .64 . The scale Instruction and Feedback was not changed. The Cronbach's alpha was .86.

In sum, the reliability analysis resulted in the not recoding of three items, the removing of two items and delivered us a questionnaire of 35 items, consisting of five factors, with Cronbach's alphas ranging from .63 to .91. 
Table 3

Descriptives (mean, standard deviation and Cronbach's alpha) for the different scales, resulting from the exploratory factor analysis

\begin{tabular}{lllll}
\hline Scale & $\boldsymbol{N}$ & $\boldsymbol{M}$ & SD & $\boldsymbol{\alpha}$ \\
\hline Learning and reflection (P1) & 10 & 3.18 & .50 & .84 \\
Instruction and feedback (P2) & 11 & 3.21 & .59 & .86 \\
The motivating supervisor (P3) & 5 & 3.56 & .78 & .91 \\
Evaluative nature of the PDP (P4) & 5 & 3.00 & .43 & .63 \\
Perceived effectiveness of the PDP (P5) & 4 & 3.23 & .59 & .64 \\
\hline
\end{tabular}

\subsection{Confirmatory factor analysis}

First, to test the hypothesized model that was found in the exploratory factor analysis, confirmatory factor analysis (CFA, Table 4) was used on another dataset (Organization 3). The hypothesized model that was found in the exploratory factor analysis had five factors that corresponded to five different scales. It was hypothesized that the different factors correlated with each other. The items for each scale function as indicators for the respective factor. One item, "I consider my Personal Development Plan as personalized" was added by Organization 3 and was expected to load on 'the Evaluative Nature of the PDP' construct.

The model showed a moderate fit to the observed data as suggested by the goodness-of-fit-indices $\left(\chi^{2} / \mathrm{df}=2.66\right.$; SRMR $=0.085$, RMSEA $\left.=0.07 ; \mathrm{CFI}=0.78\right)$. Correlations among the five factors varied from 0.44 to 0.70 , which is consistent with the theory that the factors are expected to have large positive correlations. Second, based on the largest standardized residuals, the hypothesized model was optimized: 12 items were deleted. The 12 items were deleted because of too many cross-loadings with items loading on the other factors. Items which already showed a lower factor loading during the exploratory factor analysis in particular were deleted. Furthermore, the loading of one item "I only cover what should be incorporated in the PDP" (Instruction and Feedback scale) remained insignificant and was deleted. Next, in line with the content of the items, the following correlations between items loading on the same factor, were accepted: "Not working on my competencies has an influence on the PDP-conversation", "The PDP and the related meetings stimulate me to discuss matters with my colleagues"; "When the PDPmeeting is approaching, I spend more hours on my PDP"; "I only handle what should be incorporated in the PDP" and "I consider my PDP as something personal"; "No matter what kind of feedback I do receive, I receive it too late to be useful feedback" and "If I work on my PDP, it is not clear what is expected" (see Figure 2). This resulted in a strong model fit $\left(\chi^{2} / \mathrm{df}=1.87\right.$; SRMR $=0.051$, RMSEA $=0.055$; CFI $=0.944)$. Table 5 reports the remaining latent variables and the items that load on those variables. However, because of the continuous aspect of learning and the importance of the sustained use of a PDP (Smith \& Tillema, 1998, 2001), and because of the importance of receiving feedback and handling feedback on a regular basis, we decided to keep the item "I spend time on my PDP on a regular basis" 
Chapter 5

within the Instruction and Feedback scale. This resulted in a less strong, but still strong model fit $\left(\chi^{2} / \mathrm{df}=1.99 ; \mathrm{SRMR}=0.067\right.$, RMSEA $\left.=0.059 ; \mathrm{CFI}=0.93\right)$. 


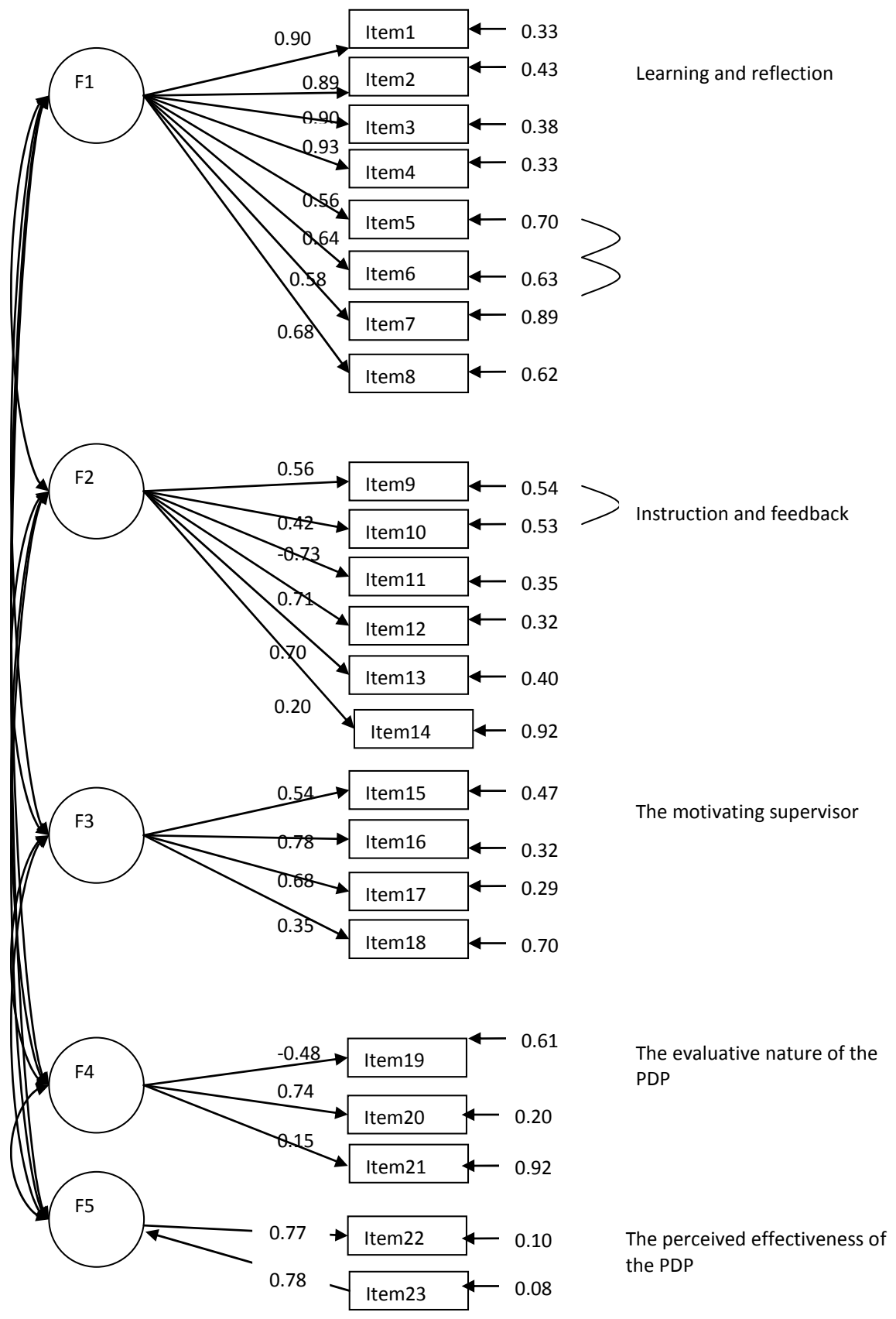

Figure 2

Confirmatory factor analysis: Factors and their items Note. Standardized Beta's are reported $(\beta)$ 
Table 4

Confirmatory factor analysis: Factors and their items

\begin{tabular}{|c|c|c|c|c|c|}
\hline Item & $\begin{array}{l}\text { Factor } \\
1\end{array}$ & $\begin{array}{l}\text { Factor } \\
2\end{array}$ & $\begin{array}{l}\text { Factor } \\
3\end{array}$ & $\begin{array}{l}\text { Factor } \\
4\end{array}$ & $\begin{array}{l}\text { Factor } \\
5\end{array}$ \\
\hline \multicolumn{6}{|l|}{ Learning and reflection } \\
\hline I learn from the PDP. & .895 & & & & \\
\hline Making the PDP really set me thinking. & .892 & & & & \\
\hline I think the PDP is interesting & .903 & & & & \\
\hline $\begin{array}{l}\text { The PDP stimulates me to discuss matters with my } \\
\text { supervisor. }\end{array}$ & .933 & & & & \\
\hline $\begin{array}{l}\text { Not working on my competencies has an influence } \\
\text { on my PDP-meeting. }\end{array}$ & .561 & & & & \\
\hline $\begin{array}{l}\text { The PDP stimulates me to discuss matters with my } \\
\text { colleagues. }\end{array}$ & .641 & & & & \\
\hline $\begin{array}{l}\text { When the PDP-meeting is approaching, I spend more } \\
\text { hours on my PDP. }\end{array}$ & .584 & & & & \\
\hline $\begin{array}{l}\text { With the feedback given, I take another close look at } \\
\text { my PDP. }\end{array}$ & .684 & & & & \\
\hline \multicolumn{6}{|l|}{ Instruction and feedback } \\
\hline $\begin{array}{l}\text { No matter what kind of feedback I am receiving, I } \\
\text { receive it too late to be useful feedback. }\end{array}$ & & .561 & & & \\
\hline $\begin{array}{l}\text { When I'm working on my PDP it is not clear what is } \\
\text { good. }\end{array}$ & & .423 & & & \\
\hline Often the feedback is given too quickly. & & -.727 & & & \\
\hline $\begin{array}{l}\text { I can not derive from the feedback what I should do } \\
\text { to improve something. }\end{array}$ & & .715 & & & \\
\hline $\begin{array}{l}\text { If I make a mistake or do not understand something, } \\
\text { I receive not much support to make sure I do it well } \\
\text { or understand it. }\end{array}$ & & .703 & & & \\
\hline I spend time on my PDP on a regular basis. & & .200 & & & \\
\hline \multicolumn{6}{|l|}{ The motivating supervisor } \\
\hline $\begin{array}{l}\text { My supervisor leaves me enough space to determine } \\
\text { the content of the PDP and the related meetings. }\end{array}$ & & & .537 & & \\
\hline $\begin{array}{l}\text { My supervisor shows understanding for my situa- } \\
\text { tion. }\end{array}$ & & & .778 & & \\
\hline $\begin{array}{l}\text { My supervisor leaves me enough choice concerning } \\
\text { the content of the PDP and the related meetings } \\
\text { (e.g. 'Which learning activities am I going to under- } \\
\text { take?). }\end{array}$ & & & .677 & & \\
\hline My supervisor can be seen as empathic. & & & .354 & & \\
\hline \multicolumn{6}{|l|}{ The evaluative nature of the PDP } \\
\hline $\begin{array}{l}\text { The feedback usually indicates how good I am doing, } \\
\text { in relation to my colleagues. }\end{array}$ & & & & -.481 & \\
\hline $\begin{array}{l}\text { When I have heard/read the feedback, I understand } \\
\text { why I received a certain grade or evaluation. }\end{array}$ & & & & .740 & \\
\hline $\begin{array}{l}\text { I consider my PDP as personalized (item added by } \\
\text { the third organization) }\end{array}$ & & & & .146 & \\
\hline
\end{tabular}


The perceived effectiveness of the PDP

While developing the PDP I learned new things.

The reliability analysis showed that the Cronbach's alpha coefficient for the Evaluative Nature of the PDP scale was -.12 and not valid. The scale was deleted. Furthermore, the analysis indicated that by deleting the item "Often the feedback is given too quickly", the Instruction and Feedback scale went from .27 to .70. As a result the Cronbach's alpha's resulting from the reliability analysis ranged from .70 to .93. Table 5 shows the alpha coefficients for the scales and descriptive statistics. In sum, the reliability analysis resulted in the deletion of the Evaluative Nature of the PDP scale and one other item.

Table 5

Descriptives (mean, standard deviation and Cronbach's alpha) for the different scales, resulting from the confirmatory factor analysis

\begin{tabular}{lllll}
\hline Scale & $\mathrm{n}$ & $\mathrm{M}$ & $\mathrm{SD}$ & $\alpha$ \\
\hline Learning and reflection (P1) & 8 & 2.79 & .81 & .89 \\
Instruction and feedback (P2) & 5 & 3.12 & .62 & .70 \\
The motivating supervisor (P3) & 4 & 3.41 & .67 & .74 \\
Perceived effectiveness of the PDP (P4) & 2 & 2.42 & .81 & .93 \\
\hline
\end{tabular}

\section{Discussion and conclusion}

Lifelong learning of employers is taking a central role in today's rapidly changing organizations. The employee's formal and informal learning can be enhanced by using a Personal Development Plan (PDP). The question is, does the PDP practice work? This study focused on the development and validation of an instrument to assess the employee's perception of the personal development plan practice within different organizational settings. To our knowledge the Personal Development Plan Practice Questionnaire (PPQ) is the first questionnaire to assess the Personal Development Plan practice in organizational settings.

The findings from the exploratory factor analysis indicated that the questionnaire consists of five factors: Learning and Reflection (10 items), Instruction and Feedback (11 items), the Motivating Supervisor (5 items), the Evaluative Nature of the PDP (5 items) and the Perceived Effectiveness of the PDP (4 items), all with satisfactory reliability and validity. Contradictory to what we expected the theoretical constructs were not found, with the exception of the Motivating Supervisorscale. This can be explained by the strong interdependence between the different scales (e.g. the three different feedback scales). A further explanation could be that the portfolio practice in educational settings is too different from the use of per- 
sonal development plans in organizations. As a consequence, using questionnaires researching the portfolio assessment practice in educational settings and translating those questionnaires to the organizational settings is difficult and in some cases impossible. For example, concerning the scale Amount and Distribution of Effort, putting a lot of effort into the portfolio as a student can be interpreted as positive. In contrast, spending too much time on your personal development plan as an employee can be interpreted as poor time-management. Furthermore, the analysis of the questionnaire indicates that filling out the form is not seen as an assessment activity per se, in contrast to the assessment interviews in which the tool is used.

Next, the confirmatory factor analysis reduced the questionnaire to 4 factors: Learning and Reflection (8 items), Instruction and Feedback (5 items), the Motivating Supervisor ( 4 items) and the Perceived Effectiveness of the PDP ( 2 items). In the last scale one item was added by the third organization. This analysis resulted in a good model fit and the deletion of 16 items. Different potential explanations can be given for the deletion of the various items. First, we assume that all items of the scale 'the Evaluative Nature of the PDP' were deleted because the scale was an intrinsic feature of the other scales or, in other words, the meaning of the scale showed a lot of overlap with the other scales (cross-loadings). Second, it is possible that the meaning of certain items was not obvious or insufficiently clear. For example, certain items were possibly formulated in a too general way (e.g. "The PDP is not really challenging" and "I have to work on my PDP regularly to get good evaluations"; What is 'a good evaluation'?). Third, based on the literature that indicates that the sustained use and the continuous aspect of learning are important (Smith \& Tillema, 1998, 2003), the item "I spend time on my PDP on a regular basis" was kept, while the confirmatory factor analysis suggested deletion of the item.

It can be argued that the Perceived Effectiveness of the PDP scale concerns an output measure, which can easily be replaced by other output measures, such as undertaking learning activities, expertise growth and performance (competencies). Those kinds of output measures can be combined with the PPQ. Nevertheless, we hypothesized that the perceived effects of the PDP would have an effect on the PDP practice as well. This is in accordance with the literature on transfer of training that indicates that seeing the training as relevant or valuing the training outcomes has a positive influence on transfer of training and/or the employees' performance (Burke \& Hutchins, 2007).

\subsection{Limitations and suggestions for future research}

The findings from this study must be carefully interpreted in line with the extent of the study's external validity. The study was conducted in three different settings, with large enough samples. Nevertheless, it is possible that the factor patterns vary when the questionnaires are filled out by different employees (e.g. experts versus 
novices), in different organizational settings (e.g. profit versus non-profit), in different disciplines/branches, with various organization sizes (small versus large scale organizations), different focuses on competencies, various learning structures and different purposes. Because of different uses, interpretations of the items may differ according to the setting. For example, it is not clear if the components are still applicable to the PDP practice if the tool is used for different goals than learning and continuing professional development, such as promotion and selection. Future research should review the factor structure of the tool in other settings and focus on assessing the effectiveness of those practices for undertaking learning activities, expertise growth and performance.

Next, the response rate in Organization 3 was low (21\%), while the response rate in Organizations 1 and 2 was $26 \%$ and $42 \%$ respectively. The procedure of data collection, however, was similar in all three organizations. The following two arguments may explain the difference in response rate. First, Organization 3 is a nonprofit organization while Organizations 1 and 2 are profit organizations, which stimulate their employees to work hard at a career and advance, and consequently positively influence the employee's motivation to make use of a PDP in order to learn and achieve that goal as well. Second, the employees in Organization 3 are older and have more work experience. For older, more experienced employees it may be less relevant to make use of a PDP.

\subsection{Implications for practice}

A literature review of articles on the effectiveness of the use of PDPs in the workplace has shown that empirical studies on the effectiveness of PDPs in the workplace are scarce. Furthermore, these studies reflect varying degrees of success of PDP practices (Kostrzewski, Dhillon, Goodsman, \& Taylor, 2009). It is crucial for Human Resource Management to optimize the PDP process.

This questionnaire, the PPQ, can be used by Human Resource Management and consultants to evaluate the PDP practice in a general way and to optimize the use. The questionnaire indicates which supporting process conditions are lacking and require more attention. This evaluation can then be used at a later stage to focus on certain conditions in more depth and to set up training for supervisors that deals with those supporting conditions that are not being paid enough attention to. Training could teach supervisors how to give good feedback, for example, or how to support the employee's learning or conduct a performance interview in which the PDP is discussed. 


\section{References}

Alder, G.S. (2007). Examining the relationship between feedback and performance in a monitored environment: A clarification and extension of feedback intervention study. Journal of High Technology Management Research, 17, 157-174.

Anseel, F., Lievens, F., \& Schollaert, E. (2009). Reflection as a strategy to enhance task performance after feedback. Organizational Behavior and Human Decision Processes, 110, 23-35.

Ashford, S. J. (1986). Feedback-seeking in individual adaptation: a resource perspective. Academy of Management Journal, 29 (3), 465-487.

Austin, Z., Marini, A., \& Desroches, B. (2005). Use of a learning portfolio for continuous professional development: A study of pharmacists in Ontario (Canada). Pharmacy Education, 5, 175-181.

Bartram, D., \& Roe, R.A. (2008). Individual and organizational factors in competence acquisition. In: W.Nijhof (Ed.). The Learning Potential of the Workplace (pp. 71-96). Rotterdam: Sense Publishers.

Beausaert, S., Segers, M., van der Rijt, J., Gijselaers, W. (2011). The use of Personal Development Plans in the workplace: A literature review. In P. van den Bossche, W. Gijselaers, \& R. Milter (Eds.), Building learning experiences in a changing world, Advances in Business Education and Training III (pp. 235-265). Dordrecht: Springer.

Bentler, P.M. (1990). Comparative fit indexes in structural models, Psychological Bulletin, 107, 238-246.

Berliner, D.C. (1984). The half-full glass: A review of research on teaching. In P.L. Hosford (ed.) Using what we know about teaching (pp. 51-77). Alexandria, Va.: Association for Supervision and Curriculum Development.

Biggs, J. (2003). Teaching for quality learning at university. Maidenhead: McGraw-Hill Education.

Bolhuis, S. (2003). Towards process-oriented teaching for self-directed lifelong learning: A multidimensional perspective. Learning and Instruction, 13, 327-347.

Bollen, K.A. (1989). Structural equations with latent variables. New York: John Wiley \& Sons.

Boud, D. (2001). Knowledge at work: Issues of learning. In D. Boud, \& S. Solomon (Eds.), Work-based learning. A new higher education? (pp.34-43). Buckingham: Open University Press.

Browne, M.W., \& Cudeck, R. (1992). Alternative ways of assessing model fit. Sociological Methods and Research, 21, 230-258.

Bullock, A., Firmstone, V., Frame, J., \& Bedward, J. (2007). Enhancing the benefit of continuing professional development: A randomized controlled study of personal development plans for dentists. Learning in Health and Social Care, 6 (1), 14-26.

Bunker, A., \& Leggett, M. (2004). Being wise about teaching portfolios: Exploring the barriers to their development and maintenance, retrieved from http://www.herdsa.org.au/wp-content/uploads/ conference/2004/PDF/P047-jt.pdf on 20/09/2007.

Burke, L.A., \& Hutchins, H.M. (2007). Training transfer: An integrative literature review. Human Resource Development Review, 6, 263-296.

Butler, D.L., \& Winne, P.H. (1995). Feedback and self-regulated learning: A theoretical synthesis. Review of Educational Research, 65 (3), 245-281.

Byrne, B.M. (1998). Structural equation modeling with lisrel, prelis, and simplis. Mahwah, NJ: Erlbaum.

Chickering, A.W., \& Gamson, Z.F. (1991) Applying the Seven Principles to Good Practice in Undergraduate Education. San Francisco: Jossey-Bass.

Driessen, E.W., van Tartwijk, J., van der Vleuten, C.P.M., \& Wass, V.J. (2007). Portfolios in medical education: Why do they meet with mixed success? A systematic review. Medical Education, 41, 12241233.

Evans, A., Ali, S., Singleton, C., Nolan, P., \& Bahrami, J. (2002). The effectiveness of personal education plans in CPD: An evaluation. Medical Teacher, 24 (1), 79-84.

Fabrigar, L.R., Wegener, D.T., MacCallum, R.C., \& Strahan, E.J. (1999). Evaluating the use of exploratory factor analysis in psychological research. Psychological Methods, 4, 272-299. 
Fenwick, T.J. (2006). Contradictions in portfolio careers: work design and client relations. Career Development International, 11, 66-79.

Firssova O. (2006). ePortfolio as a coaching support tool for workplace learning of teachers. Paper presented at ePortfolio conference October 11-13, 2006, Oxford. Retrieved from: http://www.netwerkopenhogeschool.com/Docs/Expertise/RdMC/Congresbijdragen\%202006/06_1 0_19_PaperE_portfolioincoaching.pdf on 20/09/2007.

Field, A. (2005). Discovering Statistics using SPSS; (and sex, drugs and rock ' $n$ ' rol)I ( $2^{\text {nd }}$ ed.). London: Sage Publications.

Gelderman, C.J., \& van Weele, A.J. (2002). Strategic direction through purchasing portfolio management: A case study. The Journal of Supply Chain Management, 38 (2), 30-37.

Gerbing, D.W., \& Hamilton, J.G. (1996). Viability of exploratory factor analysis as a precursor to confirmatory factor analysis. Structural Equation Modeling, 3, 62-72.

Gibbs, G., \& Simpson, C. (2004). Does your assessment support your student's learning? Journal of Learning and Teaching in Higher Education, 1, 3-21.

Gibbs, G., Simpson, C., \& MacDonald, R. (2003). Improving student learning through changing assessment - a conceptual and practical framework. Paper presented at the European Association for Research into Learning and Instruction Conference, August, Padova, Italy.

Guaglianone, C.L. (1995). Portfolio assessment of administrators. Paper presented at the Annual Meeting of the National Council of Professors of Educational Administration, $49^{\text {th }}$, Williamsburg, VA, August 8-12, 1995.

Hattie, J., \& Timperley, H. (2007). The power of feedback. Review of Educational Research, 77, 81-112.

Ilgen, D.R., Fisher, C.D., \& Taylor, M.S. (1979). Consequences of Individual Feedback on Behavior in Organizations. Journal of Applied Psychology, 64, 349-371.

Kluger, A.N., \& DeNisi, A. (1996). The effects of feedback interventions on performance: A historical review, a meta-analysis, and a preliminary feedback intervention theory. Psychological Bulletin, 119, 254-284.

Kolb, D.A. (1984). Experiential Learning. Chicago: Prentice Hall.

Kostrzewski, A. J., Dhillon, S., Goodsman, D., \& Taylor, K. M. G. (2009). The influence of continuing professional development portfolio records on pharmacy practice. The International Journal of Pharmacy Practice, 17 (2), 107-113.

Larson, J.R., Jr. (1989). The dynamic interplay between employees' feedback-seeking strategies and supervisors' delivery of performance feedback. Academy of Management Review, 14, 408-422.

Lin, C., Tan, B., \& Hsieh, P. (2005). Application of the Fuzzy Weighted Average in Strategic Portfolio Management. Decision Sciences, 36, 489-511.

London, M., Larsen, H.H., \& Thisted, L.N. (1999). Relationships between feedback and self-development. Group \& Organizational Management, 24 (1), 5-27.

London, M., \& Smither, J.W. (1999). Empowered self-development and continuous learning. Human Resource Management, 38, 3-15.

Lyons, N., \& Evans, L. (1997). Portfolio: A tool for self-directed learning at work. Paper presented at the Self-directed Learning: Past and Future Symposium (Montreal, Quebec, Canada, September 1997).

Mathers, N.J., Challis, M.C., Howe, A.C., \& Field, N.J. (1999). Portfolios in continuing medical education effective and efficient? Medical Education, 33, 521-530.

McMullan, M. et al. (2003). Portfolios and assessment of competence: A review of the literature. Journal of Advanced Nursing, 41, 283-294.

Noe, R.A. (1988). An investigation of the determinants of successful assigned mentoring relationships. Personnel Psychology, 41, 457-479.

Noe, R.A. (1996). Is Career Management Related to Employee Development and Performance? Journal of Organizational Behavior, 17, 119-133.

Prosser, M., \& Trigwell, K. (1999). Understanding learning and teaching: The experience in higher education. Buckingham: Open University Press. 
Roberts, G.E. (2003). Employee Performance Appraisal System Participation: A Technique that Works. Public Personnel Management, 32, 89-98.

Segers, M., Gijbels, D., \& Thurlings, M. (2008). The relationship between students' perceptions of portfolio assessment practice and their approaches to learning. Educational Studies, 34 (1), 35-44.

Seng, S.H., \& Seng, T.O. (1996). Reflective teaching and the portfolio approach in early childhood staff development. Paper presented at the Joint Conference of the Educational Research Association of Singapore and the Australian Association for Research in Education. Singapore, 25-29 November 1996.

Schmitz, C.C., \& Schillo, B.A. (2005). Report Carding: A model for foundation portfolio assessment. Americain Journal of Evaluation, 26, 518-531.

Shute, V. J. (2008). Focus on Formative Feedback. Review of Educational Research, 78 (1), 153-189.

Smith, K., \& Tillema, H. (1998). Evaluating portfolio use as a learning tool for professionals. Scandinavian Journal of Educational Research, 42, 193-205.

Smith, K., \& Tillema, H. (2001). Long-term influences of portfolios on professional development. Scandinavian Journal of Educational Research, 45, 183-202.

Smith, K., \& Tillema, H. (2003). Clarifying Different Types of Portfolio Use. Assessment \& Evaluation in Higher Education, 28, 625-646.

Stapleton, C.D. (1997). Basic concepts and procedures of confirmatory factor analysis. Paper presented at the Annual Meeting of the Southwest Educational Research Association (January 23-25, 1997), Austin, TX.

Steelman, L. A., Levy, P. E., \& Snell, A. F. (2004). The Feedback Environment Scale: construct definition, measurement, and validation. Educational and Psychological Measurement, 64, 165-184.

Taylor, M.S., Fisher, C.D., \& Ilgen, D.R. (1984). Individuals reactions to performance feedback in organizations. A control theory persepective. In K.M. Rowland \& G.R. Ferris (Eds.). Research in personnel and human resources management (pp. 81-124). Greenwich, CT: JAI Press.

Tigelaar, D.E.H., Dolmans, D.H.J.M., de Grave, W.S., Wolfhagen, I.H.A.P., van der Vleuten, C.P.M. (2006). Participants' opinions on the usefulness of a teaching portfolio. Medical Education, 40, 371-378.

Tillema, H.H. (1998). Design and validity of a portfolio instrument for professional training. Studies in Educational Evaluation, 24, 263-278.

Tillema, H.H. (2001). Portfolios as Developmental Assessment Tools. International Journal of Training and Development, 5, 126-135.

Tillema, H.H., Smith, K. (2000). Learning from portfolios: Differential use of feedback in portfolio construction. Studies in Educational Evaluation, 26, 193-210.

Turner, J., Mavin, S., \& Minocha, S. (2006). We will teach you the steps but you will never learn to dance. The learning organization, 13, 398-412.

Wasylyshyn, K.M. (2003). Executive Coaching: an outcome study. Consulting Psychology Journal: Practice and Research, 55, 94-106.

Wilkinson et al. (2002). The use of portfolios for assessment of the competence and performance of doctors in practice. Medical Education, 36, 918-924.

Wright, W.A., Knight, P.T., \& Pomerleau, N. (1999). Portfolio people: Teaching and learning dossiers and the future of higher education. Innovative Higher Education, 24 (2), 89-102.

Yammarino, F.J., \& Atwater, L.E. (1993). Self perception accuracy: Implications for human resource management. Human Resource Management, 32, 231-247.

Zeichner, K., \& Wray, S. (2001). The teaching portfolio in US teacher education programs: what we know and what we need to know. Teaching and teacher education, 17, 613-621. 


\section{CHAPTER 6}

\section{USING A PDP: THE ROLE OF SUPPORTING ASSESSMENT CONDITIONS ${ }^{19,20}$}

In the current search for tools which encourage and assess development, personal development plans (PDPs) are being used ever more frequently by organizations. A PDP is an assessment tool used by the employee to document the competencies $s /$ he has been working on and to present his/her plans for further development. This study focuses on the PDP practice as conceptualized by three supporting conditions: Learning and reflection, Information and feedback and the Motivating supervisor. It examines which of these features enhance the undertaking of learning activities, expertise-growth, flexibility towards changing circumstances and performance. The results indicate that a motivating supervisor, information and feedback and reflection by the employee on the basis of his/her PDP influence whether or not a PDP contributes to the three measured output variables. Next, evidence was found for the mediating role of undertaking learning activities in the relation between the PDP practice and two output variables.

\section{Introduction}

Given the highly competitive environment, for organizations it is essential to keep up and to react quickly to the dynamics of the business world. The influx of new knowledge and technologies makes it challenging to keep up. In order to keep on delivering high-quality performance, it is of vital importance to invest in the employees in terms of enabling and supporting them to continuously learn and develop. Edmondson (2008) made a good point when she argued that experimentation and reflection by the employees are vital to the success of organizations. In her view many management systems suffer from over-emphasizing efficient production, which challenges the importance of learning quickly while maintaining high-

19 Based upon Beausaert, S., Segers, M., \& Gijselaers, W. (accepted with revisions). Using a PDP: The role of supporting assessment conditions. We would like to thank Prof. Dr. Kari Smith from the University of Bergen, Norway for her fruitful comments and advices on this paper. 
quality standards. To accomplish that, organizations need to support employees' expertise development in terms of adapting to fit new parameters, new scenarios, and new challenges. As Grenier and Kehrhahn (2008, p. 199) argue: "Experts today operate within volatile territories in which the conditions change in both subtle and obvious ways, forcing experts back into development modes to adapt their competence in the setting". In this respect, Van der Heijden (2000) evidenced flexibility is a dimension of expertise development.

In addition to high quality performance and expertise development, employability is a key issue in the field of Human Resource Development. In order to meet fluctuating demands, organizations need highly employable workers. For the employee, employability enables him/her to cope with fast-changing job requirements. At the same time, given that careers are less predictable and show fewer boundaries, it fosters a successful career development. (Van der Heijde \& Van der Heijden, 2006)

In order to reach expertise development, flexibility, better employability as well as high-quality performance, employees need to undertake learning activities intentionally (van de Wiel et al., 2004). Researchers have been examining organizational conditions which foster learning (e.g. Egan, Yang, \& Bartlett, 2004; Macneil, 2001), and have been looking for tools that encourage employees to undertake intentional learning activities (van de Wiel et al., 2004). Particular attention has been paid to training design, assessment of training, and tools that encourage knowledge workers to maintain and improve their expertise (Ericsson et al., 2006; Kozlowski \& Salas, 2010). With respect to the latter, tools such as $360^{\circ}$-feedback, self- and/or peer-assessment, and Personal Development Plans (PDPs) have been argued to be successful in stimulating learning and development (e.g. Evans et al., 2002; Wildy \& Wallace, 1998). However, evidence has been showing that implementing these tools does not guarantee effectiveness in terms of fostering employees' learning and development. The tools should be facilitated by the presence of various supporting conditions, (Beausaert et al., 2011; Pham, Segers, \& Gijselaers, 2010). A review study including 54 empirical studies was recently conducted on the effectiveness of PDPs (Beausaert et al., 2011). A PDP is used in assessment cycles to present information about the competencies the employee has been working on and is planning to further develop. The findings of the review indicated that how employees react to a PDP is influenced by several conditions. First, given the reflective nature of the PDP, the extent to which the employee experiences the PDP as a learning and reflection tool, is a necessary individual condition. Second, the PDP is the basis for conversations with the supervisor or coach who provides the employee with feedback, stimulates the employee's reflection and motivates the employee to learn. Therefore, the role of the supervisor as feedback provider and motivator is a relevant contextual condition for a PDP to be effective. 
The study presented here addresses which of the aforementioned conditions in terms of features of the PDP practice are related to employees' positive reactions to this tool. Studies in the area of $360^{\circ}$-feedback (e.g., Atwater \& Brett 2005; London \& Smither, 1995) point to the importance of people's reactions to feedback in terms of cognitive and emotional evaluations, because these have great influence on how managers will ultimately respond. As Atwater and Brett (2005) argue: "The immediate reactions managers have to $360^{\circ}$-feedback are important because the ways an individual 'feels' about and reacts to the feedback may influence how or whether the individual changes his or her behavior in response to the feedback" ( $p$. 533). Therefore, in our study the reactions to PDP were conceptualized (following Atwater and Brett 2005) as the undertaking of learning activities, perceived improvement in performance, expertise development and flexibility as a result of using a PDP.

\subsection{PDPs and their purposes}

In an effort to make sure employees develop professionally, the interest of organizations in setting up assessment cycles, consisting of development-, follow-up-, and performance interviews, started to grow over the course of the last ten years (James \& Pedder, 2006). In this respect PDPs are increasingly implemented as a tool in assessment cycles. The PDP can be characterized as a tool that:

- gives an overview of the competencies the employee worked on in the past and which competencies the employee is planning to work on in the future;

- $\quad$ is composed and written by the employee himself (self-direction by the employee) although the structure of the PDP is mostly fixed;

- $\quad$ is used as a basis/structure for conversations with the supervisor or coach who provides the employee with feedback and stimulates the employee's reflection; and

- $\quad$ serves as a decision-making tool, from planning an individual training-program (formative assessment) to assessing the suitability of a promotion (summative assessment).

In the workplace PDPs are used for different purposes. A distinction has commonly been established between professional development (formative assessment) and certification/selection/accountability (summative assessment) (Smith \& Tillema, 2001). When a PDP is used to support development, reflection and feedback, learning takes a central part. Conversely, when a PDP is used for certification, presenting oneself is more important (Bunker \& Leggett, 2004; Smith \& Tillema, 2001). 


\subsection{The PDP practice: Supporting conditions}

In this study we discern three supporting conditions: The employee's learning and reflection, the motivating role of the supervisor and the information and feedback $\mathrm{s} /$ he provides.

\subsubsection{Supporting condition 1: The employee's learning and reflection}

Austin, Marini and Desroches (2005, p. 181) describe a PDP as an instrument that requires "an idealized type of individual who knows how to self-reflect, is open to change, interested in her/his own development and knows how to organize her/himself and her/his environment to support learning". Reflection is considered a key element in professional development. Reflection signifies a critical analysis of previous experiences and aims at intensifying cognitive elaboration on those experiences; it ideally eventually leads to behavioral changes (Anseel, Lievens, \& Schollaert, 2009; McMullan et al., 2003; Seng \& Seng, 1996; Smith \& Tillema, 1998). In other words, reflecting provides insight into the employee's own processes of learning (McMullan et al., 2003). This process of individual learning can best be explained by referring to Kolb's model of experiential learning, in which reflection on personal experiences plays a central role (Kolb, 1984). According to this theory learning occurs through 'concrete experiences'. Afterwards the employee reflects on what he experienced, by using a PDP. By thinking over what happened, the learner is able to draw a more general conclusion (abstraction) and build up concepts (conceptualization). Finally, the learner can use previous experiences and what he learned from those experiences as a basis for new active experimentation, and, finally, an improved performance. Research indicated that when an employee does not reflect and learn (e.g. through completing and using a PDP), s/he will not undertake subsequent learning activities. This in turn will hinder expertise-growth, including flexibility, and high-quality performance (Anseel et al., 2009; McMullan et al., 2003; Seng \& Seng, 1996; Smith \& Tillema, 1998).

In line with previous research the following work hypotheses are formulated:

- $\quad H 1$. Learning and reflecting within a PDP practice is related to employees' reaction to the PDP (PDP component 1).

- $\quad H 2$. The relation between the learning and reflection within a PDP practice and the employees' reaction to the PDP is mediated by undertaking learning activities (mediation 1).

\subsubsection{Supporting condition 2: Providing information and feedback}

Motivating employees to use PDPs and to take advantage from its use, demands clear information on how to use the tool first and feedback during the use of it second. 
Transparency is a key feature of any assessment practice. This implies that supervisors provide their employees with an explicit and clear purpose, a formal framework (including meetings), specific guidelines and standards or criteria. Many authors have argued that the effects of PDPs would be larger if employees received this information (e.g. Guaglianone, 1995; Noe, 1996; van der Heijden \& Nijhof, 2004).

In addition to providing transparent information, feedback plays a crucial role in stimulating PDP-users to undertake learning activities and to develop their competencies (e.g. Maurer, Mitchell, \& Barbeite, 2002; Tigelaar et al., 2006a). Feedback gives information about the employee's work (Ashford, 1986) with the purpose of improving learning and performance (Shute, 2008). More specifically, the three major feedback questions are: 'Where am I now?', 'Which steps to take?', and 'Where to go next?'. When a discrepancy is detected between the competencies an employee possesses and the competencies the employee should possess, learning can be stimulated (Hattie \& Timperley, 2007). In order to be effective, feedback needs to meet some requirements (Kluger \& DeNisi, 1996).

First, London and Smither (1999) argued on the importance of providing employees with nonthreatening performance feedback (London \& Smither, 1999). This means that the feedback focuses on the employee's tasks, giving constructive suggestions on what might be needed or changed to better fulfil the task's goals (Gibbs \& Simpson, 2004). In other words, feedback focuses on the employee's performance behavior and learning and not on the employee as a person or the performance compared with colleagues (Tillema \& Smith, 2000). If employees are able to link the feedback to their performance and the supervisor's expectations of their performance, the feedback becomes meaningful and employee effort will increase (Gibbs \& Simpson, 2004; Ilgen, Fisher, \& Taylor, 1979). In contrast, by focusing on the individual or comparing the employee with his or her colleagues, the employee's motivation and self-efficacy are undermined, which is undesirable since the latter is related to the employee's effort and task persistence (Gibbs \& Simpson, 2004).

Second, feedback that is not specific (e.g. "Well done", not saying what exactly was well done) induces uncertainty in responding to the feedback and requires greater cognitive processing to understand. As a consequence, the feedback is perceived as useless, evokes frustration and inhibits the employee's motivation (London, Larsen, \& Thisted, 1999; London \& Smither, 1999; Shute, 2008). Providing corrective, negative or too extensive feedback may be ineffective as well. Corrective feedback leads to resistance when given too frequently (Butler \& Winne, 1995). Negative feedback appears to be accepted and followed less easily than positive feedback and too extensive feedback inhibits the employee's feeling of control on the task (Shute, 2008; Yammarino \& Atwater, 1993), while both feelings of control and feelings of competence have to be high in order for an employee to 
be intrinsically motivated (Ilgen et al., 1979). Finally, a lack of feedback may lead to anxiety, inaccurate self-evaluations, and a diversion of effort towards feedback gathering activities (e.g. performance interviews) (Taylor, Fisher, \& Ilgen, 1984).

Third, feedback timing has been addressed in former feedback research. However, it showed conflicting results on the relationship between feedback timing and learning and performance. While many field studies found positive effects of immediate feedback, laboratory studies showed the positive results of delayed feedback (Shute, 2008).

In line with previous research the following work hypotheses are formulated:

- H3. Information and feedback given in relation to a PDP influences employees' reaction to the PDP (PDP component 2).

- $\quad$ H4. The relation between information and feedback given within a PDP practice and the employees' reaction to the PDP is mediated by undertaking learning activities (mediation 2 ).

\subsubsection{Supporting condition 3: The motivating supervisor}

Former research has been elaborating on the role of the supervisor. Based on a comparison of different types of PDPs, Tillema (2001) concludes that the power of the PDP lies in the support it provides for stimulating professional development, more than the support for certification or selection purposes. In order to stimulate the employee's professional development, the presence of an involved supervisor that provides sufficient support and leaves room for self-determination is crucial. This in turn will lead to increased intrinsic motivation, as employees experience a sense of self-competence and feel they are controlling their own behavior (Deci \& Ryan, 2000; London, Larsen, \& Thisted, 1999; London \& Smither, 1999). Therefore a supervisor should be available and have sufficient contact with the employee (Wasylyshyn, 2003). For example, the contact with the supervisor can be established by arranging fixed meetings. The lack of interaction with the supervisor is mostly due to time limitations, incompatible work schedules, and physical distance (Noe 1988). For example, research found that dentists, who received help from a supervisor [tutor] in developing a PDP, undertook more learning activities (Bullock et al., 2007). Similarly, it was found that employees are more likely to engage in developmental activities such as training when they have supervisors who are supporting their employees' efforts (Noe \& Wilk, 1993).

In line with previous research the following work hypotheses are formulated:

- H5. Having a supervisor who motivates an employee within a PDP practice is related to the employees' reaction to the PDP (PDP component 3). 
- $\quad$ H6. The relation between having a motivating supervisor within a PDP practice and the employees' reaction to the PDP is mediated by undertaking learning activities (mediation 3).

Figure 1 gives an overview of the different hypotheses.

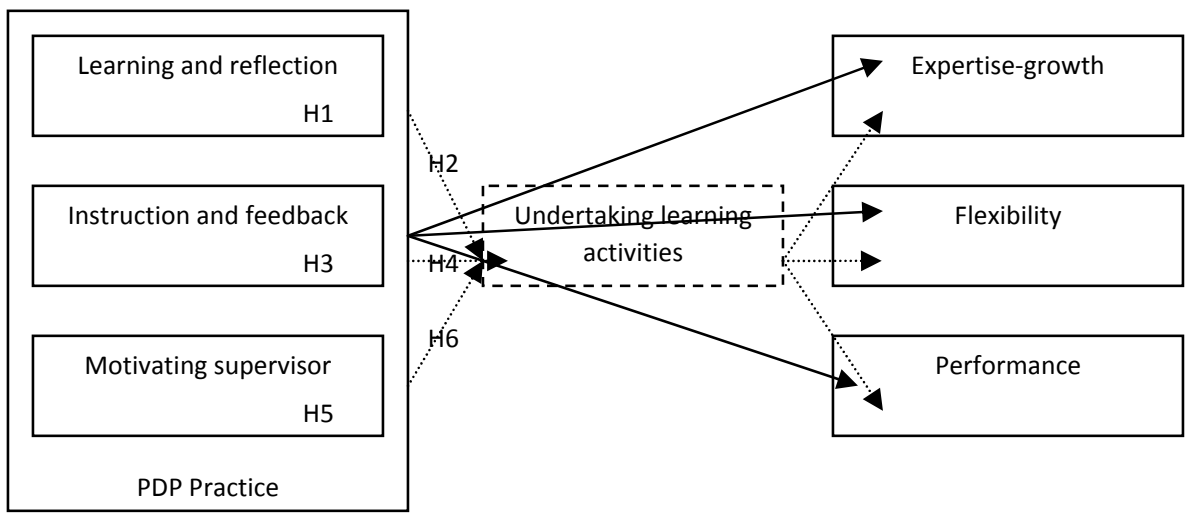

Figure 1.

Hypothetical model for the effect the PDP practice components on the outcome variables: undertaking learning activities, expertise-growth, flexibility and performance.

Note: The dashed box represents the mediating variable.

\section{Method}

\subsection{The participating organization and its employees}

Participants are employees of a regional Dutch governmental office. This office employs 1400 people in 5 different offices, located in 4 different cities. Despite the relatively large amount of employees working in different departments, the organization is perceived by the employees as a mid-sized organization. The average age of the employees was 49 in 2009 and the office has a very low turn-around of employees, both facts which make it a very stable organization.

Employees of the governmental office undergo an annual assessment cycle. The assessment cycle consists of a performance interview, a development interview and an assessment interview with the supervisor. During that assessment process the supervisor and the employee can make use of a PDP. The PDP lists the competencies the employee still needs to develop (looking forward) through evaluating his or her strengths and weaknesses (looking back). The PDP is used for various purposes, especially aiming at professional development, flexibility and mobility and certification and selection. Based on the PDP the employee and the supervisor can decide on whether or not planning a learning activity in the future. The tool is not used as strictly by every supervisor. 
Out of the 1400 employees that were contacted, a total of 287 (response rate 21\%, 187 men and 72 female; 28 missing values) participated in the research, spread over at least six different departments and four office locations. Of the 259 employees who provided us with their highest education, 27 studied WO (university), 81 HBO (non-academic higher education), 16 VWO (academically-oriented secondary education), 47 HAVO (higher secondary education), 55 MBO (secondary vocational education) and 8 VMBO (lower secondary vocational education). The average employee was 49 years old $(S D=7)$. The average number of years of experience in the organization was 21 years $(S D=12)$ and the average number of years of experience in the current role was 10 years $(S D=9)$. These numbers are in line with the statistics that are available for the total group of employees.

\subsection{Measures}

The different variables were measured with a questionnaire. The questionnaire consisted of three sections. The first section collected demographic data and the second section asked for the employee's perceptions of the PDP practice (the 3 supporting conditions). Section 3 measured employees' reaction to the PDP in terms of learning activities undertaken, expertise-growth and flexibility and improved performance. All questions were answered making use of a 5-point Likert scale, going from totally agree to totally disagree or from always to never (undertaking learning activities).

The supporting conditions of the PDP practice. To measure the employee's perception of the PDP practice we used the validated PDP Practice Questionnaire (PPQ) (Beausaert et al., 2011). The questionnaire drew inspiration from the Assessment Experience Questionnaire (AEQ) and its theoretical basis (Gibbs \& Simpson, 2004).

The questionnaire consists of the following scales: learning and reflection scale, information and feedback scale and the motivating supervisor scale. First, the learning and reflection scale measures to which extent employees perceive the reflection and learning value of a PDP. Second, the information and feedback scale asks for the employees' perception of the supervisor's guidelines on how to work with the PDP and his or her feedback in connection with the PDP. It asks whether the criteria are clear and whether the feedback is specific, timely, providing constructive suggestions and given on a regular basis. Third, the motivating supervisor scale asks for the employees' perception of the extent to which their supervisors leave room for self-determination and are involved (Deci \& Ryan, 2000; London \& Smither, 1999).

To assess the validity of the three PDP practice components, a Confirmatory Factor Analysis (CFA) was performed with the EQS 6.1 software (Bentler, 1989). The CFA showed a strong model fit for the PPQ $\left(\chi^{2} / d f=1.99 ;\right.$ SRMR $=0.067, \mathrm{RMSEA}=$ 
0.059; CFI = 0.93). Cronbach's alphas were respectively .89 for the learning and reflection scale, .77 for the information and feedback scale and .74 for the motivating supervisor scale.

\section{Employees' reactions to the PDP practice}

Undertaking learning activities. The Different types of learning activities undertaken were evaluated with the Learning Activities Scale (6 items). The scale measures to which extent employees undertake learning activities as a result of working with a PDP on a behavioral level. More specifically, on a 5-point Likert scale going from never to always it was asked how often different types of learning activities were undertaken because of the PDP (i.e. trainings, courses, workshops, conferences, intervisions, supervisions, internships and/or self-study).

Maximum Likelihood analysis resulted in one factor with item loads of .67 and higher. The component had an eigenvalue of 3.98 (corresponding to $66 \%$ of the explained variance). All items that tagged the scale Undertaking Learning Activities loaded on this component. The Cronbach's alpha was .90.

Expertise-growth and flexibility. To measure the employee's expertise-growth and flexibility toward changing circumstances, we made use of the Van der Heijden (2000) validated growth and flexibility scale (e.g. "I think it is likely that when I introduce ideas or suggestions, I show originality."). The scale ( $=19$ items) was adapted to the context of using PDPs. For example, the item "During that particular period, I concerned myself with the latest developments in the domain of my work" was reformulated as "Since I started making use of a PDP, I concern myself with the latest developments in the domain of my work".

Initially the Maximum Likelihood analysis showed the existence of three factors. However, only two items loaded on the third component. When forced to two components, the analysis with oblimin rotation resulted in two factors with item loads of .41 and higher. Eleven items loaded on the first component which had an eigenvalue of 7.26 (corresponding to $38 \%$ of the explained variance) and was labeled the employee's expertise-growth scale. Seven items loaded on the second component which had an eigenvalue of 3.30 (corresponding to $17 \%$ of the explained variance) and was labeled the employee's flexibility scale. One item had a low loading (-.28; "I think it is possible that if I did not have all the information I needed, I would feel restricted in my work" (R)) and was deleted. The Cronbach's alphas were .90 and .80 .

Performance. To measure the perceived performance we adapted the validated Output of transfer behavior scale of Xiao (1996), consisting of 6 items. Originally the scale was used to measure the effects of transfer of training. We adapted the questionnaire in order to measure employees' reaction to the PDP in terms of im- 
proved performance. For example, the item "Using the new KSA has helped me improve my work" was reformulated as "Using a PDP has helped me to improve my work".

Maximum Likelihood analysis resulted in one factor with item loads of .87 and higher. The component had an eigenvalue of 5.23 (corresponding to $87 \%$ of the explained variance). The Cronbach's alpha was .93.

For an overview of the different scales, example items, descriptives and Cronbach's alphas, we refer to Table 1.

Table 1

Overview of the different scales and their descriptives

\begin{tabular}{|c|c|c|c|c|c|}
\hline Scale & $\mathrm{N}$ & $\mathrm{M}$ & SD & $\alpha$ & Example items \\
\hline \multicolumn{6}{|l|}{ The PDP practice } \\
\hline $\begin{array}{l}\text { Learning and Reflection } \\
\text { (P1) }\end{array}$ & 8 & 2.79 & .81 & .89 & $\begin{array}{l}\text { I learn from the PDP. / Making the PDP } \\
\text { really set me thinking. }\end{array}$ \\
\hline $\begin{array}{l}\text { Instruction and Feedback } \\
\text { (P2) }\end{array}$ & 5 & 3.12 & .62 & .70 & $\begin{array}{l}\text { When I'm working on my PDP it is not } \\
\text { clear what is good. / No matter what kind } \\
\text { of feedback I am receiving, I receive it too } \\
\text { late to be useful feedback. }\end{array}$ \\
\hline $\begin{array}{l}\text { The motivating supervisor } \\
\text { (P3) }\end{array}$ & 4 & 3.41 & .67 & .74 & $\begin{array}{l}\text { My supervisor leaves me enough space to } \\
\text { determine the content of the PDP and } \\
\text { the related meetings. / My supervisor } \\
\text { leaves me enough choice concerning the } \\
\text { content of the PDP and the related meet- } \\
\text { ings (e.g. 'Which learning activities am I } \\
\text { going to undertake?). }\end{array}$ \\
\hline \multicolumn{6}{|l|}{ Outcome variables } \\
\hline $\begin{array}{l}\text { Undertaking learning } \\
\text { activities }\end{array}$ & 6 & 2.41 & .87 & .90 & $\begin{array}{l}\text { Because of using a PDP I look up things in } \\
\text { books, journal or on the internet. }\end{array}$ \\
\hline Expertise-growth & 11 & 2.63 & .61 & .90 & $\begin{array}{l}\text { Since I am using a PDP, I am focused on } \\
\text { new challenges as far as my work is } \\
\text { concerned. }\end{array}$ \\
\hline Flexibility & 7 & 3.49 & .69 & .80 & $\begin{array}{l}\text { I consider myself being able to deal with } \\
\text { future automation flexibly. }\end{array}$ \\
\hline Performance & 6 & 2.54 & .86 & .93 & $\begin{array}{l}\text { Since I am using a PDP and have related } \\
\text { meetings, the quality of my work im- } \\
\text { proved. }\end{array}$ \\
\hline
\end{tabular}

\subsection{Method of analysis}

First, descriptives and correlations between the variables were calculated. Second, we conducted hierarchical regression analyses to focus on the relative contribution of using a PDP in the prediction of employees' reaction to the PDP in terms of undertaking learning activities, expertise-growth \& flexibility, and improved performance, controlling for background variables. Third, based on the regression analysis, the mediation analyses were put forward. To verify the mediation hypotheses, 
regression analyses were executed using a procedure that is based on the four steps of Kenny, Kashy, and Bolger (1998).

\section{Results}

\subsection{Preliminary analysis}

Table 2

Correlations

\begin{tabular}{|c|c|c|c|c|c|c|c|}
\hline Variable & 1 & 2 & 3 & 4 & 5 & 6 & 7 \\
\hline 1. Learning and reflection & - & & & & & & \\
\hline 2. Instruction and feedback & $.48^{* *}$ & - & & & & & \\
\hline 3. Motivating supervisor & $.30 * *$ & $.40^{* *}$ & - & & & & \\
\hline $\begin{array}{l}\text { 4. Undertaking learning } \\
\text { activities }\end{array}$ & $.39 * *$ & $.14^{*}$ & $.20 * *$ & - & & & \\
\hline 5. Expertise-growth & $.50 * *$ & $.25^{* *}$ & $.19 * *$ & $.40 * *$ & - & & \\
\hline 6. flexibility & $.22 * *$ & .06 & $.23^{* *}$ & $.38 * *$ & $.32 * *$ & - & \\
\hline 7. Performance & $.50 * *$ & $.23^{* *}$ & $.28 * *$ & $.44^{* *}$ & $.69 * *$ & $.23 * *$ & - \\
\hline
\end{tabular}

Table 2 shows the correlations between the three subscales of the PDP practice (Learning and reflection, Information and feedback and the Motivating supervisor) and the four dependent variables (Undertaking learning activities, Expertisegrowth, Flexibility, and Performance). All scales correlate significantly and positively, except for the scale Information and feedback which did not correlate with the scales Undertaking learning activities and Flexibility.

\subsection{The influence of the PDP components on the outcome variables}

To determine the relative contribution of the independent PDP practice components in predicting the undertaking of learning activities, expertise-growth, flexibility and performance, we executed hierarchical regression analyses; controlling for the background variables gender, education, office, department (within the office), and experience in the employee's current role. The findings show that the PDP component learning and reflection (PDP component 1 ) predicts all outcome variables, the undertaking of learning activities $(\theta=.38 ; p<.001)$, expertise-growth ( 6 $=.52 ; p<.001)$, flexibility $(B=.21 ; p<.01)$, and performance $(B=.48 ; p<.001)$, significantly positively. Hypothesis 1 is confirmed (Table $3 a$ ). 
Table 3a

Hierarchical regression analyses of the learning and reflection component (PDP practice component) on the dependent variables: undertaking learning activities, expertise-growth and performance

\begin{tabular}{|c|c|c|c|c|c|c|c|c|}
\hline $\begin{array}{l}\text { PDP practice com- } \\
\text { ponent }\end{array}$ & $\begin{array}{l}\text { Undert } \\
\text { learning } \\
\text { ties }\end{array}$ & activi- & $\begin{array}{l}\text { Expertise } \\
\text { growth }\end{array}$ & & Flexibilit & & Perform & nce \\
\hline & $\beta$ & $\Delta R^{2}$ & $\beta$ & $\Delta R^{2}$ & $\beta$ & $\Delta R^{2}$ & $\beta$ & $\Delta R^{2}$ \\
\hline \multicolumn{9}{|l|}{ Step 1} \\
\hline Gender & .03 & & .07 & & -.01 & & .02 & \\
\hline Education & -.11 & & -.10 & & .08 & & $-.14^{*}$ & \\
\hline Office & .07 & & .08 & & .13 & & .11 & \\
\hline Department & -.08 & & -.06 & & -.02 & & $-.16^{*}$ & \\
\hline \multirow[t]{2}{*}{ Experience in role } & -.04 & & .01 & & -.18 & & -.02 & \\
\hline & & .02 & & .02 & & $.05^{*}$ & & .04 \\
\hline \multicolumn{9}{|l|}{ Step 2} \\
\hline Gender & -.03 & & -.02 & & -.04 & & -.06 & \\
\hline Education & -.12 & & -.11 & & .07 & & $-.15^{* *}$ & \\
\hline Office & .02 & & .01 & & .10 & & .05 & \\
\hline Department & -.00 & & .05 & & .02 & & -.06 & \\
\hline Experience in role & .01 & & .06 & & -.16 & & .03 & \\
\hline \multirow{2}{*}{$\begin{array}{l}\text { Learning and } \\
\text { reflection }\end{array}$} & $.38 * * *$ & & $.52 * * *$ & & $.21^{* *}$ & & $.48^{* * *}$ & \\
\hline & & $.13^{* * *}$ & & $.25 * * *$ & & $.04 * * *$ & & $.21^{* * *}$ \\
\hline Total $\mathbf{R}^{2}$ & & $.15^{* * *}$ & & $.27 * * *$ & & $.09 * * *$ & & $.25^{* * *}$ \\
\hline
\end{tabular}

Note. The reported regression coefficients are standardized coefficients.

${ }^{*} p<.05,{ }^{* *} p<.01,{ }^{* * *} p<.001$.

Furthermore, Instruction and feedback (PDP component 2 ) predicts the employee's expertise-growth $(B=.22 ; p<.01)$ and performance $(b=.20 ; p<.01)$ significantly positively. The component does not predict the undertaking of learning activities ( $B$ $=.11 ; n s)$ or flexibility $(B=-.00 ; n s)$ significantly. It can be concluded that information and feedback given in relation to the PDP is beneficial for the employee's expertise-growth and performance. Since Information and feedback does not predict the two other outcome variables, hypothesis 2 is only partially confirmed (Table 3b). 
Table 3b

Hierarchical regression analyses of the information and feedback component (PDP practice component) on the dependent variables: undertaking learning activities, expertise-growth and performance

\begin{tabular}{|c|c|c|c|c|c|c|c|c|}
\hline \multirow[t]{2}{*}{$\begin{array}{l}\text { PDP practice com- } \\
\text { ponent }\end{array}$} & \multicolumn{2}{|c|}{$\begin{array}{l}\text { Undertaking } \\
\text { learning activi- } \\
\text { ties }\end{array}$} & \multicolumn{2}{|c|}{$\begin{array}{l}\text { Expertise- } \\
\text { growth }\end{array}$} & \multicolumn{2}{|c|}{ Flexibility } & \multicolumn{2}{|c|}{ Performance } \\
\hline & $\beta$ & $\Delta R^{2}$ & $\beta$ & $\Delta R^{2}$ & $\beta$ & $\Delta R^{2}$ & $\beta$ & $\Delta R^{2}$ \\
\hline \multicolumn{9}{|l|}{ Step 1} \\
\hline Gender & .03 & & .07 & & -.01 & & .02 & \\
\hline Education & -.11 & & -.10 & & .08 & & $-.14^{*}$ & \\
\hline Office & .07 & & .08 & & $.13^{*}$ & & .11 & \\
\hline Department & -.08 & & -.06 & & -.02 & & $-.16^{*}$ & \\
\hline \multirow[t]{2}{*}{ Experience in role } & -.04 & & .01 & & $-.18^{* *}$ & & -.02 & \\
\hline & & .02 & & .02 & & $.05^{*}$ & & .04 \\
\hline \multicolumn{9}{|l|}{ Step 2} \\
\hline Gender & .01 & & .04 & & -.01 & & -.01 & \\
\hline Education & -.11 & & -.10 & & .08 & & $-.15^{*}$ & \\
\hline Office & .06 & & .06 & & .13 & & .09 & \\
\hline Department & -.06 & & -.01 & & -.02 & & -.11 & \\
\hline Experience in role & -.02 & & .03 & & $-.18^{* *}$ & & .00 & \\
\hline \multirow{2}{*}{$\begin{array}{l}\text { Information and } \\
\text { feedback }\end{array}$} & .11 & & $.22^{* *}$ & & -.00 & & $.20 * *$ & \\
\hline & & .01 & & $.04 * *$ & & .00 & & $.04^{* *}$ \\
\hline Total $\mathbf{R}^{2}$ & & .03 & & $.06 * *$ & & $.05^{*}$ & & $.08^{* *}$ \\
\hline
\end{tabular}

Note. The reported regression coefficients are standardized coefficients.

$* p<.05,{ }^{* *} p<.01,{ }^{* * *} p<.001$.

Finally, similar to the learning and reflection component, the motivating supervisor (PDP component 3 ) predicts all outcome measures, undertaking learning activities $(\beta=.22 ; p<.01)$, expertise-growth $(\beta=.19 ; p<.01)$, flexibility $(\beta=.21 ; p<.01)$ and performance $(\beta=.30 ; p<.001)$, significantly positively. Therefore, it can be concluded that when the supervisor supports and motivates the employee within a PDP practice, the employee will undertake learning activities, show expertisegrowth and flexibility and an improved performance. Hypothesis 3 is confirmed (Table 3c). 
Table 3c

Hierarchical regression analyses of the motivating supervisor component (PDP practice component) on the dependent variables: undertaking learning activities, expertise-growth and performance

\begin{tabular}{|c|c|c|c|c|c|c|c|c|}
\hline $\begin{array}{l}\text { PDP practice com- } \\
\text { ponent }\end{array}$ & $\begin{array}{l}\text { Undert } \\
\text { learnin } \\
\text { ties }\end{array}$ & activi- & $\begin{array}{l}\text { Expertise } \\
\text { growth }\end{array}$ & & Flexibili & & Perform & nce \\
\hline & $\beta$ & $\Delta R^{2}$ & $\beta$ & $\Delta R^{2}$ & $\beta$ & $\Delta R^{2}$ & $\beta$ & $\Delta \mathrm{R}^{2}$ \\
\hline \multicolumn{9}{|l|}{ Step 1} \\
\hline Gender & .03 & & .07 & & -.01 & & .02 & \\
\hline Education & -.11 & & -.10 & & .08 & & $-.14^{*}$ & \\
\hline Office & .07 & & .08 & & $.13^{*}$ & & .11 & \\
\hline Department & -.08 & & -.06 & & -.02 & & $-.16^{*}$ & \\
\hline \multirow[t]{2}{*}{ Experience in role } & -.04 & & .01 & & $-.18^{* *}$ & & -.02 & \\
\hline & & .02 & & .02 & & $.05^{*}$ & & .04 \\
\hline \multicolumn{9}{|l|}{ Step 2} \\
\hline Gender & .03 & & .07 & & -.01 & & .02 & \\
\hline Education & -.13 & & -.11 & & .06 & & $-.16^{*}$ & \\
\hline Office & .05 & & .06 & & .10 & & .08 & \\
\hline Department & -.07 & & -.05 & & -.01 & & $-.14^{*}$ & \\
\hline Experience in role & -.00 & & .04 & & $-.15^{*}$ & & .03 & \\
\hline \multirow{2}{*}{$\begin{array}{l}\text { Motivating super- } \\
\text { visor }\end{array}$} & $.22 * *$ & & $.19 * *$ & & $.21 * *$ & & $.30 * * *$ & \\
\hline & & $.04 * *$ & & $.03 * *$ & & $.04 * *$ & & $.09 * * *$ \\
\hline Total $\mathbf{R}^{2}$ & & $.06 * *$ & & $.05 * *$ & & $.09 * *$ & & $.13^{* * *}$ \\
\hline
\end{tabular}

Note. The reported regression coefficients are standardized coefficients.

${ }^{*} p<.05,{ }^{* *} p<.01,{ }^{* * *} p<.001$.

\subsection{The mediating role of the extent to which employees undertake learning activities}

First, the mediating role of the undertaking of learning activities between the learning and reflection component (PDP component 1 ) and the three outcome variables (expertise-growth, flexibility and performance) was researched. To that end 3 different mediation analyses were conducted. As mentioned in the preliminary analysis, there is a significant and positive relation between learning and reflection (independent variable) and the outcome variables (dependent variables) (step 1). In step 2 we found a significant relation between the independent variable and the undertaking of learning activities (mediating variable). In step 3 we found a significant relation between the mediating variable (undertaking learning activities) and the dependent variables (expertise-growth, flexibility and performance), while controlling for the independent variable. When the mediating variable was taken into account in step 4, we found a significant decrease of the relation between learning and reflection and expertise-growth and between learning and reflection and performance. The decrease of the relation between learning and reflection and flexibility was not significant. The Sobel test (Sobel, 1982) showed that the indirect 
effect of learning and reflecting by using a PDP through undertaking learning activities on expertise-growth and performance was significant. Therefore, we can conclude that the relation between the learning and reflecting within a PDP practice and the employee's expertise-growth and performance is partially mediated by the undertaking of learning activities (Table 4). This result partially confirms hypothesis 4.

Table 4

Mediation analysis with the PDP component learning and reflection as independent variable

\begin{tabular}{|c|c|c|c|c|c|c|c|c|}
\hline $\begin{array}{l}\text { Independent } \\
\text { variable: }\end{array}$ & Mediator & $\begin{array}{l}\text { Dependent } \\
\text { variable }\end{array}$ & Step 1 & Step 2 & Step 3 & Step 4 & Sobel & $\begin{array}{l}R \text { - } \\
\text { square }\end{array}$ \\
\hline \multirow[t]{3}{*}{$\begin{array}{l}\text { Learning } \\
\text { and reflec- } \\
\text { tion }\end{array}$} & $\begin{array}{l}\text { Undertaking } \\
\text { learning } \\
\text { activities }\end{array}$ & $\begin{array}{l}\text { Expertise- } \\
\text { growth }\end{array}$ & $.50 * * *$ & $.38 * * *$ & $.24 * * *$ & $.41^{* * *}$ & $3.73^{* * *}$ & $.30 * * *$ \\
\hline & & Flexibility & $.22^{* * *}$ & $.38 * * *$ & $.35^{* * *}$ & .08 & $4.48^{* * *}$ & $.15^{* * *}$ \\
\hline & & Performance & $.50 * * *$ & $.38 * * *$ & $.29 * * *$ & $.39 * * *$ & $4.29 * * *$ & $.32 * * *$ \\
\hline
\end{tabular}

Note: Step 1 = Pad from the independent variable (learning and reflectioin) to the dependent variable (expertise-growth, flexibility or performance). Step 2 = Pad from the independent variable to the mediating variable (undertaking learning activities). Step $3=$ Pad from the mediating variable to the dependent variable (controlled for the independent variable). Step $4=$ Pad from the independent variable to the dependent variable (controlled for the mediating variable).

$* p<.05, * * p<.01, * * * p<.001$.

Second, the mediating role of the undertaking of learning activities between the motivating supervisor component (PDP component 3) and the outcome variables (expertise-growth, flexibility and performance) was researched. These analyses led to similar results as the previous mediation analyses. It can be concluded that the relation between the motivating supervisor within a PDP practice and the employee's expertise-growth and performance is partially mediated by the undertaking of learning activities (Table 5). This result confirms hypothesis 5 partially.

Table 5

Mediation analysis with the PDP component the motivating supervisor as independent variable

\begin{tabular}{|c|c|c|c|c|c|c|c|c|}
\hline $\begin{array}{l}\text { Independent } \\
\text { variable }\end{array}$ & Mediator & $\begin{array}{l}\text { Dependent } \\
\text { variable }\end{array}$ & Step 1 & Step 2 & Step 3 & Step 4 & Sobel & $\begin{array}{l}R \text { - } \\
\text { square }\end{array}$ \\
\hline \multirow[t]{3}{*}{$\begin{array}{l}\text { Motivating } \\
\text { supervisor }\end{array}$} & $\begin{array}{l}\text { Undertaking } \\
\text { learning act. }\end{array}$ & $\begin{array}{l}\text { Expertise- } \\
\text { growth }\end{array}$ & $.19 * *$ & $.20 * *$ & $.37 * * *$ & $.11^{*}$ & $3.13^{* *}$ & $.17^{* * *}$ \\
\hline & & Flexibility & $.23 * * *$ & $.20 * *$ & $.35 * * *$ & $.16^{* *}$ & $3.07^{* *}$ & $.17^{* * *}$ \\
\hline & & Performance & $.28 * * *$ & $.20 * *$ & $.40 * * *$ & $.19 * * *$ & $3.19 * *$ & $.23^{* * *}$ \\
\hline
\end{tabular}

Note: Step 1 = Pad from the independent variable (motivating supervisor) to the dependent variable (expertise-growth, flexibility or performance). Step 2 = Pad from the independent variable to the mediating variable (undertaking learning activities). Step $3=$ Pad from the mediating variable to the dependent variable (controlled for the independent variable). Step $4=$ Pad from the independent variable to the dependent variable (controlled for the mediating variable).

$* p<.05, * * p<.01, * * * p<.001$. 
The information and feedback component did not predict the undertaking of learning activities significantly. For that reason, no mediations were calculated with the information and feedback component as independent variable.

\section{Conclusion and discussion}

The results of this study indicate that if the employee perceives the PDP as a reflection and learning tool, and if the supervisor is perceived as motivating, the employee is far more likely to react positively to the PDP by undertaking more learning activities, showing more expertise-growth and flexibility, and reporting improved performance.

First, while we researched the PDP practice in an organizational context, our results are in line with previous research conducted within a school setting (students). Tillema (2001) stated: "Putting PDPs [portfolios] to use essentially means setting the goals for learning and reflection first". The supervisor can motivate the employee in this by creating a learning environment where the learner takes control over his/her learning. The supervisor communicates the goals of the use of the tool, introduces in which way it should be used, supports and motivates the employee, has meetings with the employee on a regular basis, is autonomysupportive, takes care of the follow-up and gives high-quality feedback. Next, Tillema (2001) for example found reflective portfolios, focusing on documenting professional growth and determining learning goals, to be effective for supporting learning and a better performance. Furthermore, Tigelaar and colleagues (2006b) found a positive relation between using a PDP and reflection in teachers. However, the research showed that this reflection is often not very profound and teachers do not easily reflect on their motivation, feelings, thoughts and their personality. The support of a coach, colleague or supervisor who stimulates the reflection is necessary, for example during a formal professional development meeting every six months.

Second, contrary to our hypothesis, the results show that, although the information and feedback given within a PDP practice is related to the employee's expertise-growth and performance, there is no significant relation with the extent to which employees undertake learning activities and how flexible $s /$ he is. Various explanations for these contrasting findings are explored in literature. Of specific interest are the studies on the effects of $360^{\circ}$-feedback. For example, the study of London and Smither (1995) showed that the characteristics of the feedback recipients (attitudes, personality or self-efficacy) influence how the one that receives the feedback reacts to and uses it. In relation to this study, one possible hypothesis is that undertaking learning activities and flexibility may be more dependent on the recipient's characteristics. Additionally, the feedback employees receive from their 
supervisors, which we addressed in our study, might be more focused on the employee's development and performance than on supporting employees in undertaking learning activities.

Third, the mediation hypotheses concerning the partial mediation of undertaking learning activities between the learning and reflection component and the dependent variables (expertise-growth and performance), and between the motivating supervisor and the dependent variables, were confirmed. This confirms former research of for example Davis et al. (1999) who found that when employees participate in activating learning activities, this can effect change in professional practice of physicians. Moreover, these results are in line with research evidencing the role of the supervisor in organising a positive learning climate where employees are stimulated to work on their professional development (Noe \& Wilk, 1993).

Fourth, in contrast to our hypothesis, no partial mediation effect was found for undertaking learning activities between learning and reflection and flexibility and between the motivating supervisor and flexibility. However, we believe that flexibility might be explained by whether or not having conducted various tasks in your job which might be a more powerful predictor of flexibility than the undertaking of learning activities. Therefore supervisors need to organize the environment in such a way that employees can learn from a variety of tasks in their job (Van der Heijden, 2000).

\subsection{Implications for future research}

First, Smith and Tillema (1998) indicated that, to have high quality PDPs, it is important that one uses the tool for a longer period of time, since that influences the effects positively. Longitudinal research could shed more light on this matter.

Second, this research focused on one organization. It is relevant for future research to validate our findings across different organizations. Supporting conditions and effects may differ according to organizational learning culture (Marsick \& Watkins, 2003), sectors or disciplines (e.g. Datta, Guthrie, \& Wright, 2005), type of organizations (market-oriented or not; Baker \& Sinkula, 1999), type of employees (e.g. experienced versus non-experienced) and organization sizes (small versus large scale; Saru, 2007). Often larger organizations show a more sophisticated human resource management (Guthrie, 2001).

Third, in this study, we measured employees' reactions to the PDP. A next step in the research on PDPs should focus on the extent to which these reactions lead to change in behaviour and in employability in terms of mobility. However, if you would be interested in researching employees' behavior in terms of PDP use, learning activities, expertise development and performance should be studied using observable measures. In this respect, there is a plea for using multi-raters methods and for example question peers and supervisors as well. However, using multi- 
raters methods is probably not the solution. For example, Jellema and colleagues (2006) conducted three quasi-experimental studies and found that multisource feedback is not an efficient method for training evaluation.

Fourth, the organization indicated that the tool is used for both learning/development and certification/selection purposes. The question is if using a PDP for both purposes might have influenced employees' reactions. Research indicated that in case of using a PDP for selection purposes employees' self-protection and fear of underachieving may lead to the collection of unauthentic evidence and the construction of invalid PDPs (Smith \& Tillema, 1998; 2001). This in turn might jeopardize the employees' reactions to a PDP in that sense that $s /$ he does not use the PDP as a tool for his/her professional development. Research on assessment argued that formative (learning/development purposes) and summative assessment (certification/selection purposes) should not be combined and conducted by the same supervisor (e.g. Wolf \& Dietz, 1998). Therefore we suggest for follow-up research to take into account the nature of the purposes of the PDP as perceived by the employee, as well as how this influences the PDP's effectiveness.

Fifth, no partial mediation effect was found for undertaking learning activities between learning and reflection and flexibility and between the motivating supervisor and flexibility. Future research should look into the variety of tasks the employee fulfilled in the past and its effects on flexibility.

Finally, for deepening our understanding of how the PDP practice affects the employees' reactions to the tool, we recommend the additional use of qualitative research methods such as interviews.

\subsection{Implications for practice}

This study has a few implications for human resource development in organizations. This study undermines the sceptics in a lot of organizations by showing employees can react positively to PDP assessment in case supporting conditions are perceived. As an organization it is important to measure employees' perceptions of the PDP practice since employees need to perceive the PDP in such a manner so that they react positively to it. In case employees do not perceive the PDP as a tool which stimulates their reflection and learning, causes need to be researched and changes in the implementation of the PDP need to be made. A reason can be that the PDP is used for both learning/development purposes and certification/selection purposes at the same time. An organization scores high on the Information and feedback scale if the organization communicates explicit and clearly in relation to the PDP. The criteria and the format used should be clear. Next, feedback meetings need to be explicitly planned. Finally, the supervisor (and the format) needs to provide the employee with sufficient freedom in order to make the employee feel responsible for his/her learning. It is important to find a balance between guiding 
and providing the employee with a structure on the one hand and leaving enough choices to the employee in order s/he can personalize the PDP on the other. In that way using a PDP will contribute to the undertaking of learning activities and, consequently, expertise-growth and flexibility and an improved performance.

The supervisor plays a pivotal role in this process, by supporting the learning and reflection by the employee. During meetings in which the supervisor discusses the employee's PDP with the employee him/herself, the most attention should go to the learning of the employee and stimulating his or her reflection on the learning process. Based on the results of this study, we suggest to set up trainings in which supervisors are brought together to introduce them to the underlying theory of the assessment cycles and the related tools. It is only when the supervisors are aware of the underlying theory and the assumptions that it makes, that they will be able to make them explicit to their employees. Next, participating in collective training will give them the opportunity to learn from each other. By making the supervisors talk about how they introduce the PDP to their employees and how they conduct the performance meetings, they will become aware of good and bad ways of handling this. Consequently, the assessment process and the use of PDPs as part of the continuing process of performance evaluations will be more effective. 


\section{References}

Anseel, F., Lievens, F., \& Schollaert, E. (2009). Reflection as a strategy to enhance task performance after feedback. Organizational Behavior and Human Decision Processes, 110, 23-35.

Ashford, S. J. (1986). Feedback-seeking in individual adaptation: a resource perspective. Academy of Management Journal, 29, 465-487.

Atwater, L.E., \& Brett, J.F. (2005). Antecedents and consequences of reactions to developmental $360^{\circ}$ feedback. Journal of Vocational Behavior, 66, 532-548.

Austin, Z., Marini, A., \& Desroches, B. (2005). Use of a learning portfolio for continuous professional development: A study of pharmacists in Ontario (Canada). Pharmacy Education, 5, 175-181.

Baker, W.E., \& Sinkula, J.M. (1999). The synergistic effect of market orientation and learning orientation on organizational performance. Journal of the Academy of Marketing Science, 27, 411-427.

Beausaert, S., Segers, M., van der Rijt, J., Gijselaers, W. (2011). The use of Personal Development Plans in the workplace: A literature review. In P. van den Bossche, W. Gijselaers, \& R. Milter (Eds.), Building learning experiences in a changing world, Advances in Business Education and Training III (pp. 235-265). Dordrecht: Springer.

Bentler, P.M. (1989). EQS structural equations program manual. Los Angeles: BM Software.

Bullock, A., Firmstone, V., Frame, J., \& Bedward, J. (2007). Enhancing the benefit of continuing professional development: A randomized controlled study of personal development plans for dentists. Learning in Health and Social Care, 6 (1), 14-26.

Bunker, A., \& Leggett, M. (2004). Being wise about teaching portfolios: Exploring the barriers to their development and maintenance, retrieved from http://www.herdsa.org.au/wp-content/uploads /conference/2004/PDF/P047-jt.pdf, on 20/09/2007.

Butler, D.L., \& Winne, P.H. (1995). Feedback and self-regulated learning: A theoretical synthesis. Review of Educational Research, 65 (3), 245-281.

Datta, D.K., Guthrie, J.P., \& Wright, P.M. (2005). Human Resource Management and labor productivity: Does industry matter? Academy of Management Journal, 48, 135-145.

Davis, D.M.D., Thomson O'Brien, M.A., Freemantle, N., Wolf, F.M., Mazmanian, P. \& Taylor-Vaisey, A. (1999). Impact of formal continuing medical education. Do conferences, workshops, round, and other traditional continuing education activities change physician behaviour or health care outcomes? American Medical Association, 282, 867-874.

Deci, E. L. \& Ryan, R. M. (2000). The "What" and "Why" of Goal Pursuits: Human Needs and the SelfDetermination of Behavior. Psychological Inquiry: An International Journal for the Advancement of Psychological Theory, 11 (4), 227-268.

Edmondson, A.C. (2008). The competitive imperative of learning. Harvard Business review, 86 (7/8), 6067.

Egan, T.M., Yang, B., Bartlett, K.R. (2004). The effects of organizational learning culture and job satisfaction on motivation to transfer learning and turnover intention. Human Resource Development Quarterly, 15, 279-301.

Ericsson, A., Charness, N., Feltovich, P.J., \& Hoffman, R.R. (2006). Cambridge Handbook of Expertise and Expert Performance. Cambridge: Cambridge University Press.

Evans, A., Ali, S., Singleton, C., Nolan, P., \& Bahrami, J. (2002). The effectiveness of personal education plans in CPD: An evaluation. Medical Teacher, 24 (1), 79-84.

Gibbs, G., \& Simpson, C. (2004). Does your assessment support your student's learning? Journal of Learning and Teaching in Higher Education, 1, 3-21.

Grenier, R.S., Kehrhahn, M. (2008). Toward an integrated model of expertise redevelopment and its implications for HRD. Human Resource Development Review, 7, 198-217.

Guaglianone, C.L. (1995). Portfolio assessment of administrators. Paper presented at the Annual Meeting of the National Council of Professors of Educational Administration, $49^{\text {th }}$, Williamsburg, VA, August 8-12, 1995. 
Guthrie, J.P. (2001). High-Involvement Work Practices, Turnover, and Productivity: Evidence from New Zealand. The Academy of Management Journal, 44, 180-190.

Hattie, J., \& Timperley, H. (2007). The power of feedback. Review of Educational Research, 77, 81-112.

Ilgen, D.R., Fisher, C.D., \& Taylor, M.S. (1979). Consequences of individual feedback on behaviour in organizations. Journal of Applied Psychology, 64, 349-371.

James, M., \& Pedder, D. (2006). Beyond method: Assessment and learning practices and values. The Curriculum Journal, 17, 109-138.

Jellema, F., Visscher, A., \& Scheerens, J. (2006). Measuring change in work behavior by means of multisource feedback. International Journal of Training and Development, 10, 121-139.

Kenny, D. A., Kashy, D. A., \& Bolger, N. (1998). Data analysis in social psychology. In D. Gilbert, S. Fiske, G. Lindzey (Eds.), The handbook of social psychology (4 ${ }^{\text {th }}$ ed., Vol. 1, pp. 233-265). Boston, MA: McGraw-Hill.

Kluger, A.N., \& DeNisi, A. (1996). The effects of feedback interventions on performance: A historical review, a meta-analysis, and a preliminary feedback intervention theory. Psychological Bulletin, 119, 254-284.

Kolb, D.A. (1984). Experiential Learning. Chicago: Prentice Hall.

Kozlowski, S.W., Salas, E. (2010). Learning, Training, and Development in Organizations. New York: Routledge.

London, M., Larsen, H.H., \& Thisted, L.N. (1999). Relationships between feedback and self-development. Group \& Organizational Management, 24 (1), 5-27.

London, M., \& Smither, J.W. (1995). Can multi-source feedback change perceptions of goal accomplishments, self-evaluation and performance-related outcomes? Personnel Psychology, 48, 803-839.

London, M., \& Smither, J.W. (1999). Empowered self-development and continuous learning. Human Resource Management, 38, 3-15.

Macneil, C. (2001). The supervisor as a facilitator of informal learning in work teams. Journal of workplace learning, 13, 246-253.

Marsick, V.J., \& Watkins, K.E. (2003). Demonstrating the value of an organization's learning culture: The dimensions of the learning organization questionnaire. Advances in Developing Human Resources, 5, 132-151.

Maurer, T.J., Mitchell, D.R.D., \& Barbeite, F.G. (2002). Predictors of attitudes toward a 360-degree feedback system and involvement in post-feedback management development activity. Journal of Occupational and Organizational Psychology, 75, 87-107.

McMullan, M. et al. (2003). Portfolios and assessment of competence: A review of the literature. Journal of Advanced Nursing, 41, 283-294.

Noe, R.A. (1988). An investigation of the determinants of successful assigned mentoring relationships. Personnel Psychology, 41, 457-479.

Noe, R.A. (1996). Is Career Management Related to Employee Development and Performance? Journal of Organizational Behavior, 17, 119-133.

Noe, R.A.,\& Wilk, S.L. (1993). Investigation of the factors that influence employees' participation in development activities. Journal of Applied Psychology, 78, 291-302.

Pham, N.T.P., Segers, M.S.R. \& Gijselaers, W.H. (2010). Understanding Training Transfer Effects from a Motivational Perspective: A Test of MBA Programs. Business Leadership Review, 7, 1-25.

Saru, E. (2007). Organisational learning and HRD: how appropriate are they for small firms? Journal of European Industrial Training, 31, 36 - 51.

Seng, S.H., \& Seng, T.O. (1996). Reflective teaching and the portfolio approach in early childhood staff development. Paper presented at the Joint Conference of the Educational Research Association of Singapore and the Australian Association for Research in Education. Singapore, 25-29 November 1996.

Shute, V. J. (2008). Focus on Formative Feedback. Review of Educational Research, 78 (1), 153-189.

Smith, K., \& Tillema, H. (1998). Evaluating portfolio use as a learning tool for professionals. Scandinavian Journal of Educational Research, 42, 193-205. 
Smith, K., \& Tillema, H. (2001). Long-term influences of portfolios on professional development. Scandinavian Journal of Educational Research, 45, 183-202.

Sobel, M. E. (1982). Asymptotic intervals for indirect effects in structural equations models. In S. Leinhart (Ed.), Sociological methodology 1982 (pp. 290-312). San Francisco: Jossey-Bass.

Taylor, M.S., Fisher, C.D., \& Ilgen, D.R. (1984). Individuals reactions to performance feedback in organizations. A control theory persepective. In K.M. Rowland \& G.R. Ferris (Eds.). Research in personnel and human resources management (pp. 81-124). Greenwich, CT: JAI Press.

Tigelaar, D.E.H., Dolmans, D.H.J.M., de Grave, W.S., Wolfhagen, I.H.A.P., van der Vleuten, C.P.M. (2006a). Participants' opinions on the usefulness of a teaching portfolio. Medical Education, 40, 371-378.

Tigelaar, D.E.H., Dolmans, D.H.J.M., De Grave, W.S., Wolfhagen, I.H.A.P., \& Van der Vleuten, C.P.M. (2006b). Portfolio as a tool to stimulate teachers' reflections. Medical Teacher, 28, 277-282.

Tillema, H.H. (2001). Portfolios as Developmental Assessment Tools. International Journal of Training and Development, 5, 126-135.

Tillema, H.H., Smith, K. (2000). Learning from portfolios: Differential use of feedback in portfolio construction. Studies in Educational Evaluation, 26, 193-210.

Van der Heijde, C., \& Van der Heijden, B.I.J.M. (2006). A competency-based and multidimensional operationalization and measurement of employability. Human Resource Management, 45, 449-476.

Van der Heijden, B.I.J.M. (2000). The development and psychometric evaluation of a multi-dimensional measurement instrument of professional expertise. High Ability Studies. The Journal of the European Council for high ability, 11, 9-39.

Van der Heijden, B.I.J.M., \& Nijhof, A.H.J. (2004). The value of subjectivity: Problems and prospects for 360-degree appraisal systems. International Journal of Human Resource Management, 15, 493-511.

Van de Wiel, M.W.J., Szegedi, K.H.P., \& Weggeman, M.C.D.P. (2004). Professional learning: Deliberate attempts at developing expertise. In: H.P.A. Boshuizen, R. Bromme, H. Gruber (Eds.) Professional Learning: Gaps and transitions on the way from novice to expert (pp. 181-206). Dordrecht, the Netherlands: Kluwer.

Wasylyshyn, K.M. (2003). Executive Coaching: an outcome study. Consulting Psychology Journal: Practice and Research, 55, 94-106.

Wildy, H., \& Wallace, J. (1998). Professionalism, portfolios and the development of school leaders. School Leadership and Management, 18 (1), 123-140.

Wolf, K., \& Dietz, M. (1998). Teaching portfolios: Purposes and possibilities. Teacher Educational Quarterly, 25 (1), 9-22.

Xiao, J. (1996). The relationship between organizational factors and the transfer of training in the electronics industry in Shenzhen, China. Human Resource Development Quarterly, 7, 55-72.

Yammarino, F.J., \& Atwater, L.E. (1993). Self perception accuracy: Implications for human resource management. Human Resource Management, 32, 231-247. 


\section{CHAPTER 7}

\section{General conclusion and discussion}

Within the context of implementing effective staff development strategies, organizations use a variety of assessment (appraisal or review) methodologies that have the aim of promoting both the professional and personal growth of individuals in the workplace, encompassing techniques such as formal performance ratings, multi-source feedback techniques, and personal development plans (McDowall \& Fletcher, 2004). In general a PDP can be described as an assessment tool embedded in a larger assessment cycle of development and performance interviews, used to gather and document information about the competencies the employee worked on and is planning to further develop. The PDP is used in the hope that employees would intentionally undertake learning activities and in turn improve workplace performance (London, 1997; van de Wiel, Szegedi, \& Weggeman, 2004). Although there is a trend towards using the tool for performance appraisal (summative assessment), the power of the tool lies in supporting employees' professional development (formative assessment) (Darling-Hammond \& Snyder, 2000; Smith \& Tillema, 2003). Questions which are central in the PDP are: How would you evaluate your competencies? What are your strengths and weaknesses? How are you going to work on your weaknesses (learning activities)? Did you reach your learning goal (result) or what is the status? The questions are meant to stimulate the employee's reflection on his/her competencies. When an employee reflects on his/her strengths and weaknesses, s/he becomes aware of the competencies s/he still needs to work on in order to reach a certain purpose. In order to fill the gap, the employee starts undertaking learning activities of different kinds (e.g. reading a book, looking something up on the internet, having discussions with colleagues, looking for a mentor, doing a course or training), in order to improve his or her performance.

In this concluding chapter we bring together the studies which have been discussed in this PhD-thesis by firstly discussing previous research on PDPs. Secondly, we will 
sum up and relate the findings of the studies described in this book. Finally, we will elaborate on the practical implications and make a couple of suggestions for future research.

\section{Beyond popularity: Looking for empirical evidence}

In the past a great deal has been written on Personal Development Plans or portfolio assessment (the latter term is found the most in literature). This has been proven by the literature review, discussed in Chapter 2. The review indicated that the search term portfolio (in title) which was looked for in ten different databases resulted in more than $\mathbf{3 0 0 0}$ hits. Furthermore, the search terms 'portfolio (title) and organization' resulted in $\mathbf{5 8 0}$ hits. However, most of the literature is situated within an educational context (students or teachers) or in health service (general practitioners, nurses, dentists, pharmacy assistants or therapists). In addition, most of the literature is descriptive, describing different cases in which a PDP is effectively used. Empirical evidence on its impact or effects is scarce (e.g. Austin, Marini, \& Desroches, 2005; Evans, Ali, Singleton, Nolan, \& Bahrami, 2002; Zeichner \& Wray, 2001). For the literature review of empirical research on the use of Personal Development Plans, 54 relevant references were retrieved. The lack of empirical research on this topic might be explained by the fact that PDP assessment has been implemented in organizations in the course of the last decennium only. Furthermore, previous studies on PDPs have focused on small groups of PDP users (e.g. Tigelaar, Dolmans, De Grave, Wolfhagen, van der Vleuten, 2006) and have the disadvantage of not being suitable to generalize the results to other settings. Next, to date hardly any studies on the effects of PDPs have used a control group design and therefore no conclusions could be drawn on the effectiveness of PDPs. Moreover, the results of the empirical studies are inconclusive. Most studies indicate that PDP assessment is effective for learning, personal or professional development and improving professional practice, but others (e.g. Bunker and Leggett, 2005; Little \& Hayes, 2003) do not support these positive findings. It is argued, however hardly evidenced, that the effectiveness of PDP assessment is dependent on certain supporting process conditions (e.g. the support of a supervisor). In sum, despite the popularity of the tool, there is a gap in the research on the effectiveness of PDPs and the supporting conditions which make the tool effective. This PhD-thesis wants to fill that gap and contribute to the optimization of PDP practices to enhance their effectiveness. Therefore, we have systematically researched three questions: (1) Is the PDP effective for the undertaking of learning activities and the employee's performance? (2) Which goals is a PDP used for and how does the employee's perception of those goals influence the employee's undertaking of learning activities and performance? (3) Does the PDP practice, characterized by three supporting 
conditions (the employee's learning and reflection, the instruction and feedback given by the supervisor, and the way the supervisor motivates the employee) influence the undertaking of learning activities, expertise-growth and performance? In other words, firstly we question whether using a PDP is effective. Secondly, we research if the perceived purpose and supporting conditions influence the effectiveness of using a PDP for the undertaking of learning activities, expertise-growth and consequently, performance.

\section{Results: Empirical evidence on the effects, purposes and support- ing conditions of PDPs}

In this paragraph we will formulate an answer to the three main research questions. We will argue the results based on the insights from the four studies included in this thesis.

\subsection{Is the PDP effective for the undertaking of learning activities and the em- ployee's performance?}

While it is assumed that when the employee works on his or her competencies necessary for the job by using a PDP, his or her performance will improve, the literature review (Chapter 2 ) reported inconclusive research findings. While five studies indicated ineffectiveness for learning, providing evidence, reflection or documenting, the other studies mostly indicated effectiveness for personal or continuing professional development $(\mathrm{N}$ studies $=17$ ), stimulating reflection ( $\mathrm{N}$ studies $=$ 18 ) and improving professional practice or performance ( $N$ studies $=5$ ). Most studies were conducted by using qualitative research methods $(\mathrm{N}=51)$ or a combination of qualitative and quantitative research methods $(N=23)^{21}$. More specifically, interviews ( $\mathrm{N}$ studies $=27$ ), portfolio analysis ( $\mathrm{N}$ studies $=16$ ) and focus groups $(\mathrm{N}$ studies $=5$ ) were the most used qualitative research methods. In contrast, questionnaires ( $\mathrm{N}$ studies $=23$ ) and surveys ( $\mathrm{N}$ studies $=13$ ) were used less, although they were the most used quantitative methods. Next, previous studies on PDPs are often based on small sample sizes (e.g. Tigelaar, et al., 2006) and therefore do not lead to generalizable results. Furthermore, to date, hardly any studies on the effects of PDP's have used a control group design and therefore could not draw valid conclusions on the effectiveness of PDPs. Therefore a quasi-experimental study was set up (Chapter 3), comparing PDP and non-PDP users in the extent to which they participate in training activities - learning activities in the past, planning fur-

21 The 23 studies are included in the sample of 51 qualitative studies as well. 
ther learning activities - and the competence development. The study was based on a large data set of pharmacy assistants $(N=2271)$.

It was found that users of a PDP undertook more learning activities (in the past) than non-users. However, in contrast to our expectations, pharmacy assistants who use a PDP did not plan more learning activities. They do not plan to undertake further training more often than non PDP users. This finding might indicate that PDPs are especially used as feedback tools and not as feed forward tools. In other words, it is expected that the tools are often used for looking back, to discuss learning activities that have been undertaken. They do not serve as a tool to look forward and to support employees in the planning of future learning activities. However, in comparison to other assessment tools such as 360 degree-feedback, which put an emphasis on reviewing past performance, the PDP also incorporates the opportunity to look forward at learning and development (Tillema \& Smith, 2000).

With respect to the extent to which pharmacy assistants are competent in their job, we have found no significant differences between users and non-users of a PDP. This lack of difference might be explained by the fact that by using a PDP, the pharmacy assistants are more aware of their competencies which are needed to fulfil their job, exactly because the PDP stimulates them to think about those. This critical self-reflection on the competencies PDP-users possess might have influenced their rating in the way that they underrated themselves. As a result of this, possible differences between both groups might have disappeared.

A second explanation could be the lack of reflection skills. Former research has evidenced that reflection, a vital process for professional development when working with a PDP, is still an unfamiliar skill for PDP users (Smith \& Tillema, 1998). As a consequence, it is possible that PDP users did not show a significant improvement in job competencies because of the lack of reflection and in turn the lack of connection between self-reflection and practice improvement (Austin, et al., 2005; Orland-Barak, 2005).

Third, it might be questioned to what extent the lack of effects of PDP use on learning and job competencies is context-specific. Results can differ according to the organizational learning culture (Marsick \& Watkins, 2003), sectors or disciplines (e.g. Lee, 2003), type of organizations (market-oriented or not; Baker \& Sinkula, 1999), type of employees (e.g. experienced versus non-experienced) and organization sizes (small versus large scale). Often larger organizations show a more sophisticated human resource management (Guthrie, 2001). 


\subsection{Which goals is a PDP used for and how does the employee's perception of those goals influences the employee's undertaking of learning activities and per- formance?}

The literature review (Chapter 2) indicated that the PDP is used for different purposes. A difference is commonly made between professional development and certification/selection/accountability (Smith \& Tillema, 2001). When a personal development plan is used for professional development, learning takes a central role. Conversely, when a PDP is used for certification, presenting oneself is more important. In addition, the literature review resulted in the identification of nine clusters of goals. On the one hand the following learning and development purposes were identified: (1) professional development; (2) reflective learning; (3) coaching; (4) stimulating confidence; and (5) organizing. On the other hand the following certification and selection purposes were clustered: (6) providing evidence; (7) documenting; (8) certification, selection and promotion; (9) external mobility. In Chapter 4 we researched the influence of the perceived purpose of a PDP on the undertaking of learning activities and performance among experts from an international organization that specializes in medical technology and employees of a regional Dutch governmental organization. The results indicated that although PDPs are valid tools for performance appraisal, its power lies in supporting employees in their professional development. More concretely, the use of a PDP stimulates employees to reflect on the extent to which they possess the competencies necessary for their job and on the learning activities undertaken to enhance the level of proficiency of the job competencies. Furthermore, PDPs lead to a diagnosis of the gaps in job competencies and stimulate employees to plan further learning activities.

These results imply that in order to make employees undertake learning activities and improve their performances by using a PDP, introducing and using the PDP as a tool for learning and development is the most effective. To that end, the supervisor needs to make the learning and development purpose of the PDP explicit to the employee and support the use as a learning tool with appropriate guidance. However, the fact that the perception of the tool as a certification and selection tool also predicts the undertaking of learning activities and employees' performance positively, leads to the question: How to balance between certification and selection purposes on the one hand and learning and development purposes on the other, knowing that learning and development purposes are stronger predictors of undertaking learning activities and performance? For example, Brutus, London and Martineau (1999) researched 360 degree-feedback and its relation with setting development goals. They found that ratings given by supervisors are less important than feedback received from subordinates and peers. Moreover, it seems that subordinates and colleagues are better able to influence an employee's learning and development, than the rating given by the supervisor. Keeping this in 
mind, supervisors could focus on using the PDP as a certification and selection tool, while the tool could be discussed with peers in order to reach the learning and development purposes.

\subsection{Do three supporting conditions - the employee's learning and reflection, the instruction and feedback given by the supervisor, and the way the supervisor motivates the employee - influence the undertaking of learning activities, exper- tise-growth and performance?}

In the study discussed in Chapter 6 we researched which conditions enhance the effectiveness of PDP assessment. The literature review on PDP assessment in the workplace (Chapter 2) indicated that in order to optimize the PDP assessment process in organizations, several supporting conditions need to be present. A distinction is made between conditions related to how the PDP is embedded in the environment (contextual supporting conditions) and those related to the user of the personal development plan (individual supporting conditions).

In order to measure the supporting conditions' influence on the PDP practice, we have developed and validated the PDP Practice Questionnaire (PPQ). The validation study (Chapter 5) was conducted in three different organizations: among employees of an international organization that specializes in global business services, experts from an international organization that specializes in medical technology and employees of a regional Dutch governmental organization. The study evidenced the construct validity of the questionnaire and showed a 3-factor structure: Learning and reflection ( 8 items, $\alpha=.89$ ), Instruction and feedback (5 items, $\alpha=.70$ ) and the motivating supervisor ( 4 items, $\alpha=.74$ ). First, the learning and reflection scale measures to which extent employees appreciate the reflection and learning value of a PDP. Second, the instruction and feedback scale asks for the employees' appreciation of the supervisor's guidelines on how to work with the PDP and his or her feedback in connection with the PDP. It asks whether the criteria are clear and whether the feedback is specific, timely, providing constructive suggestions and given on a regular basis. Third, the motivating supervisor scale asks for the employees' perception of the extent to which their supervisors leave room for taking initiative and are involved, both of which influence the employee's motivation positively (Deci \& Ryan, 2000).

Addressing the influence of the three aforementioned conditions, a study among employees in a government office (Chapter 6) proved that if the employee reflects on the PDP and learns from it and if the supervisor is perceived as motivating, the employee is far more likely to undertake more learning activities, show more expertise-growth and flexibility towards changing circumstances, and perform better. In sum, indications for the effectiveness of the PDP as an assessment tool can be found in the way the assessment process is set up and organized and/or 
in the presence of various supporting process conditions (Learning and reflection and Motivating supervisor). Although we researched the PDP practice in an organizational context, our results are in line with the previous research conducted within a school setting (students), from Bullock et al. (2007), Tillema (2001) and Tigelaar and colleagues (2006b). In contrast to what we hypothesized in our study, the instruction and feedback that are given did not seem to play a crucial role. This may possibly explained by the fact that many supervisors find it hard to give the right feedback in a good way. How feedback is given by supervisors is still a major concern. Next, professionals are reluctant to receive feedback from their supervisor (Smith \& Tillema, 2003). Using $360^{\circ}$-feedback, for example, in which colleagues (and customers) also participate in the feedback process, could be a solution to this problem (Brutus et al., 1999).

In addition, our results indicate that the effect of perceiving a PDP as a learning and reflection tool on expertise-growth and performance is stronger when employees undertake more learning activities. Moreover, the undertaking of learning activities mediates the effect of the perception of a motivating supervisor and the dependent variables. This illustrates that using a PDP as learning and reflection tool leads towards the undertaking of learning activities, which in its turn leads to expertise-growth and a better performance. Taking together these findings and the results of the study researching the purposes of assessment (Chapter 4), it can be concluded that when employees perceive the PDP as a learning and development tool and also use the PDP in that way, positive effects on the undertaking of learning activities and performance are to be expected. Next, and in line with the previous research of London et al. (1999), having a supervisor that motivates the employee in the assembly and use of a PDP has a positive effect on the undertaking of learning activities and consequently positively influences the employee's expertisegrowth and performance.

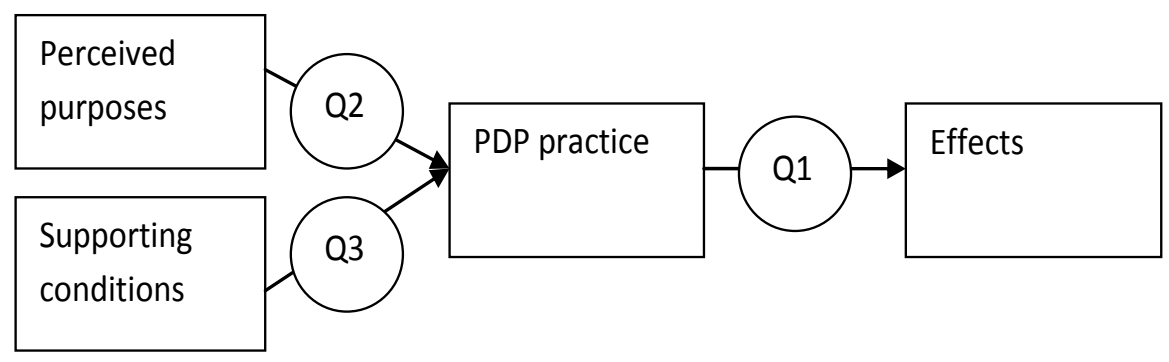

Figure 1

Overview and integration of the three main research questions

In sum, bringing the three answers to the three different research questions together (Figure 1); it can be concluded that PDP users generally undertook more learning activities in the past, but that apparently using a PDP is not effective for 
planning future learning activities when used by pharmacy assistants. Furthermore, it does not seem to be effective in order to improve general and specific job competencies of pharmacy assistants. However, in order to optimize the use of the tool, the purpose and the supporting conditions have to be taken into account. As found by a study conducted in a governmental organization in the Netherlands and a company specialized in medical technology, perceiving the tool as a learning and development tool is the strongest predictor of the undertaking of learning activities and performance, in contrast to perceiving it as a selection and certification tool. Next, the presence of a motivating supervisor, who stimulates the employee's reflection by giving clear instructions and high-quality feedback, may positively influence the effects of using a PDP within a governmental organization.

\section{Practical implications for Human Resource Management and De- velopment}

This study has a few implications for human resource management and development departments, for supervisors who are supporting employees in using a PDP and for employees who are using a PDP. Table 1 gives an overview of the practical implications for all three parties.

First, in order to make employees undertake learning activities and improve their performance, it is important to make the purpose of the PDP explicit and use it accordingly. Introducing and using a PDP is the most effective when it is used for developmental purposes. In that case, it supports employees to undertake learning activities and consequently improve their performance. Our results indicate that when employees perceive the tool as a certification and selection tool, however, it also predicts their undertaking of learning activities and performance although to a lesser extent. This leads to the question: How to balance between certification and selection purposes on the one hand and learning and development purposes on the other hand, knowing that learning and development purposes are stronger predictors of undertaking learning activities and performance? First, it is important to make the distinction between the two purposes explicit. This can be done by, for example, conducting learning and development interviews explicitely separate from performance interviews. Furthermore, different raters can be used for appraisal (selection and certification purposes) and review techniques (learning and development purposes). For PDPs with a developmental purpose, the use of multiple sources such as the employee himself, peers and subordinates is more powerful, because those are more powerful sources of feedback and feedforward information than supervisors. 
Second, it was found that using the PDP as a reflection tool is a crucial step towards learning and development. However, the results of our study among pharmacy assistants using a PDP confirms the findings of Smith and Tillema (1998) that reflection has been proved to be an unfamiliar skill for PDP-users. PDP-users find it hard to reflect on their strengths and weaknesses and to formulate the competencies they still need to work on. Therefore, it is important that supervisors or coaches help their employees with reflecting, for example during interviews in which the PDP is discussed, so that employees can translate their new knowledge into practice. The supervisors or coaches also need to help their employees in connecting reflection with practice improvement further (Austin et al., 2005; Orland-Barak, 2005). During meetings in which the supervisor discusses the employee's PDP with the employee him/herself, the most attention should go to the future learning of the employee and stimulating his or her reflection on the past learning process. In this way, the supervisor acts as a motivator and a stimulator.

Based on the implications formulated above, we suggest to set up workshops in which supervisors are brought together to introduce them to the underlying theory of the assessment cycles and the related tools. It is only when the supervisors are aware of the underlying theory and assumptions, that they will be able to make them explicit to their employees. Next, participating in a collective program will give them the opportunity to learn from each other. By making the supervisors talk about how they introduce the PDP to their employees and how they conduct the performance meetings, they will become aware of the strengths and the pitfalls, too, of this tool. Consequently, the assessment process and the use of PDPs as part of the continuing process of performance appraisals will become more effective.

Third, the results indicated that the pharmacy assistants use a PDP as a feedback tool especially, while the tool could add significant value to the learning and development process of the employee if it would be used as a feed-forward instrument as well. In other words, the tool should more often be used in order to get an overview of desired future plans, plan future careers, and plan future learning activities in order to reach set goals.

Finally, the PDP Practice Questionnaire (Chapter 5) can be used as an evaluation tool by Human Resource Management and consultants to monitor and optimize the PDP practice. When administering the instrument to all involved in the PDPpractice, the results can indicate the extent to which supporting process conditions (Learning and reflection, Instruction and feedback, Motivating supervisor) are present and where improvements can be made. The results could be used to organize training programs to support supervisors as well as employees in the optimisation of the use of PDP's as a developmental tool. 
In table 1, we summarize the critical steps to take to effectively implement a PDP. We categorized the steps in a sequential order, starting with the first step, i.e. the definition of the purpose of using a PDP.

Table 1

Steps to take when implementing a PDP

... for Human Resource Management and Development
1. What is the purpose of using a PDP?

2. How does the PDP fit within the organizational strategy?

3. How will the PDP be implemented within the organization?

4. How does the PDP form will look like?

5. Are the supporting conditions present?
- Learning and development

- Selection and certification

- Combination of both; importance of finding a balance between the two purposes - Aging employees, innovations and competition

- Reorganizations

- Selection of high-potentials

- Performance appraisal

- When is the PDP discussed?

- How often is the PDP discussed?

- Who is the PDP discussed with? (coach versus supervisor)

- Which questions are asked?

1. How would you evaluate your competencies?

2. What are your strengths and weaknesses?

3. How are you going to work on your weaknesses (which learning activities are you going to undertake)?

4. Did you reach your learning goal (result) or what is the status?

- Is the purpose, the rationale and the set-up of the PDP communicated to the supervisors and the employees?

- Does the employee reflect and learn?

- Is the employee given the responsibility to fill in the PDP (autonomy-support)? - Is there a motivating supervisor or coach who guides the employee and stimulates reflection?

- Are the supervisors trained in supporting the use of PDPs? 


\begin{tabular}{l} 
tool to the employee \\
2. Find a balance between \\
learning and development \\
purposes and selection and \\
certification purposes \\
3. Not only use the tool to look \\
back at learning activities \\
undertaken, but also to look \\
forward at future plans. What \\
are the employee's future \\
aspirations and which compe- \\
tencies does s/he need to work \\
on? \\
4. Motivate and support the \\
employee by stimulating his or \\
her reflection (focus on the \\
reflection questions). Help the \\
employee with reflecting. \\
1. Take initiative, prepare the \\
PDP interview and fill in the \\
PDP yourself. \\
2. Use the questions on the \\
PDP form to think about your \\
learning and development and \\
future career. \\
\hline for employees
\end{tabular}

\section{What is next?}

This research project is a step in building empirical evidence on the effectiveness of PDPs and on the role of different conditions to enhance that effectiveness. Future research can take this project a few steps further.

First, the concept of the PDP has many connotations in literature as well as in practice. In order to enhance comparability, only literature and practices in accordance with our definition of a PDP (Chapter 2) were taken into account. Future research should not only focus on different definitions and purposes of PDPs, but also on the different formats that are used. Some formats include a proficient analysis of strengths and weaknesses, for instance, while others only focus on defining goals, the way in which the goals are going to be reached and in which timeframe. Furthermore, some PDPs already indicate the competencies needed for the job profile, while others do not indicate any competency. Next, the implementation and procedure of using and discussing the tool vary a lot between companies. For example, the number of formal meetings in which the PDP is discussed differ. Future research should research the effectiveness of the different procedures. 
Table 2

Comparing the organizational characteristics of the organizations involved in the research project

\begin{tabular}{|c|c|c|c|}
\hline $\begin{array}{l}\text { Organization 1: Phar- } \\
\text { macy assistants } \\
\text { (Chapter } 3 \text { ) }\end{array}$ & $\begin{array}{l}\text { Organization 2: Gov- } \\
\text { ernment offices (Chap- } \\
\text { ter } 4,5 \text { and } 6 \text { ) }\end{array}$ & $\begin{array}{l}\text { Organization 3: Medi- } \\
\text { cal technology (Chap- } \\
\text { ter } 4 \text { and 5) }\end{array}$ & $\begin{array}{l}\text { Organization 4: Global } \\
\text { Business Services } \\
\text { (Chapter 5) }\end{array}$ \\
\hline Profit & Non-profit & Profit & Profit \\
\hline Small & Large & Large & Large \\
\hline $\begin{array}{l}\text { No career perspec- } \\
\text { tives }\end{array}$ & $\begin{array}{l}\text { No career perspec- } \\
\text { tives }\end{array}$ & Career perspectives & Career perspectives \\
\hline$?$ & $\begin{array}{l}\text { Not well implemented } \\
\text { in formal assessment } \\
\text { system (recently } \\
\text { reorganized) }\end{array}$ & $\begin{array}{l}\text { Well implemented in } \\
\text { formal assessment } \\
\text { system ( } 5 \text { years) }\end{array}$ & $\begin{array}{l}\text { Well implemented in } \\
\text { formal assessment } \\
\text { system ( } 5 \text { years) }\end{array}$ \\
\hline$M_{\text {age }}=39.54$ & $\begin{array}{l}M_{a g e}=49 \text { (aging work- } \\
\text { force) }\end{array}$ & $M_{\text {age }}=35-41$ & $M_{\text {age }}=36-40$ \\
\hline
\end{tabular}

Second, industry specific studies such as ours have a great advantage, in that they can focus on a rather homogeneous workforce. Furthermore, it allows us to control for sectoral differences in terms of labour market features (aging, mobility, employability, selection...). The four empirical studies discussed in this book were conducted in four different organizations. Although we did not conduct comparative studies, the comparison of the results obtained in those four settings provides us with indications of possible differences in PDP use related to the type of organization. The differences between the organizations might be explained as follows (Table 2). First, Organization 2 is a non-profit organization. This means that the organization does not compete with other companies and therefore is not forced to keep up with the newest developments. Furthermore, learning and development does not always play a central role in the different departments in Organization 2. In contrast, the other organizations are profit organizations. This entails that these organizations are more dynamic organizations which have to keep up with the newest developments in order to compete with other companies. As a result, learning and development is part of the organizational culture and always very present. Second, Organization 1 concerns employees working in different small firms. In small-sized organizations the need for formal assessment systems which structure and support the continuing professional development of employees is less necessary because the employee has a close relationship with the supervisor, which serves to replace the formal control system. Consequently, informal training is preferred here (Saru, 2007) and PDPs which often focus on formal learning are not always perceived as necessary and effective. In contrast, Organizations 2, 3 and 4 are large organizations in which a more formal assessment system is necessary to structure the learning and development of employees. However, PDPs do not seem to have the effect they are supposed to have. Whether or not supporting conditions are present may already explain part of the difference between an effective and an ineffective use of a PDP. Third, government offices (Organization 2) are 
more stable and bureaucratic organizations in which the skills and knowledge that employees need are less dynamic. Career perspectives are also limited, which means competition between employees is scarce and knowledge is not seen as a competitive advantage. In contrast, Organizations 1 and 3 are situated in the pharmaceutical and medical sector and Organization 4 is situated in the global business services business. These are sectors in which knowledge evolves fast (industry dynamism; Datta, Guthrie, \& Wright, 2005). Next, they provide much more career perspectives in which learning and having expertise is perceived as a competitive advantage. In Organization 3 for example it was found that employees perceive the tool as a tool for certification and selection especially and employees there undertake significantly more learning activities than those in Organization 2 (Chapter 4). Moreover, in Organization 3 the PDP is part of a better implemented assessment system in which it performs a central task: the use of the tool heavily influences whether or not an employee is selected for promotion. Fourth, the average age of the employees working in Organization 2 is 49 . This means that a large group of the employees could already be considered experts, who are no longer interested in learning and developing systematically (Cleveland, \& Shore, 1992). In contrast, Organization 1, 3 and 4 have a younger staff; the average age is between 35 and 41. It is likely that a larger group of novices is interested in learning and developing and consequently making promotion or working on their employability. All of these findings and suggestions need to be further reproduced in other industries for validation. Future research should investigate the effectiveness of PDPs for the undertaking of learning activities and job performance in a broader sample which covers different sectors and professions, ages, levels of expertise and career possibilities. Furthermore, supporting conditions and effects can differ according to organizational learning culture (Marsick \& Watkins, 2003), sectors or disciplines (e.g. Datta et al., 2005), type of organizations (market-oriented or not; Baker \& Sinkula, 1999), type of employees (e.g. experienced versus non-experienced) and organization sizes (small versus large scale; Saru, 2007). Often larger organizations show more sophisticated human resource management (Guthrie, 2001).

Third, it has been demonstrated that a high quality PDP can only be expected after being used for a longer period of time (Smith \& Tillema, 1998). The organizations discussed in this book have been using the tool for different periods of time. Organization 2 has been using the tool for a few years already and recently came up with an updated format. Organization 3 and 4 have been using the tool for five years. In line with Smith and Tillema (1998) it was found that the employees of Organization 3 undertook significantly more learning activities than the employees working in Organization 2. However, similar studies within a longitudinal design could measure the effectiveness of the PDP in the workplace more accurately. This research design would also allow us to draw conclusions about causality. 
Fourth, since it has been demonstrated that assessment practices have an impact on learning results by influencing employees' perception (Biggs, 2003), we believe questioning the employees' perception is the best way to measure the effects of assessment. However, for those interested in researching employees' behavior in terms of PDP use, learning and performance, it would be advisable to involve multi- raters and for example question supervisors as well.

In conclusion, it is not the instrument itself that makes employees learn and develop; it is the way it is implemented and used that make the assessment practice work, no matter which assessment tool is used. Research should focus on the assessment practices and supporting conditions within those practices. Assessment tools like PDPs should be used as learning and development tools especially, since their real power lies in that purpose. 


\section{References}

Austin, Z., Marini, A., \& Desroches, B. (2005). Use of a learning portfolio for continuous professional development: A study of pharmacists in Ontario (Canada). Pharmacy Education, 5, 175-181.

Baker, W.E., \& Sinkula, J.M. (1999). The synergistic effect of market orientation and learning orientation on organizational performance. Journal of the Academy of Marketing Science, 27, 411-427.

Biggs, J. (2003). Teaching for quality learning at university. Maidenhead: McGraw-Hill Education.

Brutus, S., London, M., \& Martineau, J. (1999). The impact of 360-degree feedback on planning for career development. Journal of Management Development, 18, 676-693.

Bullock, A., Firmstone, V., Frame, J., \& Bedward, J. (2007). Enhancing the benefit of continuing professional development: A randomized controlled study of personal development plans for dentists. Learning in Health and Social Care, 6 (1), 14-26.

Bunker, A. \& Leggett, M. (2005). Teaching portfolios: rhetoric, reality and reflection (unpublished).

Cleveland, J., \& Shore, L. (1992). Self- and supervisory perspectives on age and work attitudes and performance. Journal of Applied Psychology, 77, 469-484.

Darling-Hammond, L., \& Snyder, J. (2000). Authentic assessment of teaching in context. Teaching \& Teacher Education, 16, 523-545.

Datta, D.K., Guthrie, J.P., \& Wright P.M. (2005). Human Resource Management and labor productivity: Does industry matter? Academy of Management Journal, 48, 135-145.

Deci, E. L. \& Ryan, R. M. (2000). The "What" and "Why" of Goal Pursuits: Human Needs and the SelfDetermination of Behavior. Psychological Inquiry: An International Journal for the Advancement of Psychological Theory, 11 (4), 227-268.

Evans, A., Ali, S., Singleton, C., Nolan, P., \& Bahrami, J. (2002). The effectiveness of personal education plans in CPD: An evaluation. Medical Teacher, 24 (1), 79-84.

Guthrie, J.P. (2001). High-Involvement Work Practices, Turnover, and Productivity: Evidence from New Zealand. The Academy of Management Journal, 44, 180-190.

Lee, C.H., \& Bruvold, N.T. (2003). Creating Value for Employees: Investment in Employee Development. International Journal of Human Resource Management, 14, 981-1000.

Little, P., \& Hayes, S. (2003). Continuing professional development (CPD): GPs' perceptions of postgraduate education-approved (PGEA) meetings and personal professional development plans (PDPs). Family Practice, 20 (2), 192-198.

London, M. (1997). Job feedback: Giving, seeking, and using feedback for performance improvement. Mahwah, NJ: Erlbaum.

London, M., Larsen, H.H., \& Thisted, L.N. (1999). Relationships between feedback and self-development. Group \& Organizational Management, 24 (1), 5-27.

Marsick, V.J. \& Watkins, K.E. (2003). Demonstrating the value of an organization's learning culture: The dimensions of the learning organization questionnaire. Advances in Developing Human Resources, 5, 132-151.

McDowall, A., \& Fletcher, C. (2004). Employee development: An organizational justice perspective. Personnel Review, 33: 8-29.

Orland-Barak, L. (2005). Portfolios as evidence of reflective practice: What remains "untold". Educational Research, 47 (1), 25-44.

Saru, E. (2007). Organisational learning and HRD: how appropriate are they for small firms? Journal of European Industrial Training, 31 (1), 36 - 51.

Smith, K., \& Tillema, H. (1998). Evaluating portfolio use as a learning tool for professionals. Scandinavian Journal of Educational Research, 42, 193-205.

Smith, K., \& Tillema, H. (2001). Long-term influences of portfolios on professional development. Scandinavian Journal of Educational Research, 45, 183-202.

Smith, K., \& Tillema, H. (2003). Clarifying different types of portfolio use. Assessment \& Evaluation in Higher Education, 28, 625-648. 
Tigelaar, D.E.H., Dolmans, D.H.J.M., de Grave, W.S., Wolfhagen, I.H.A.P., van der Vleuten, C.P.M. (2006). Participants' opinions on the usefulness of a teaching portfolio. Medical Education, 40, 371-378.

Tigelaar, D.E.H., Dolmans, D.H.J.M., De Grave, W.S., Wolfhagen, I.H.A.P., \& Van der Vleuten, C.P.M. (2006b). Portfolio as a tool to stimulate teachers' reflections. Medical Teacher, 28, 277-282.

Tillema, H.H., \& Smith, K. (2000). Learning from portfolios: Differential use of feedback in portfolio construction. Studies in Educational Evaluation, 26, 193-210.

Tillema, H.H. (2001). Portfolios as developmental assessment tools. International Journal of Training and Development, 5 (2), 126-135.

Van de Wiel, M.W.J., Szegedi, K.H.P., \& Weggeman, M.C.D.P. (2004). Professional learning: Deliberate attempts at developing expertise. In: H.P.A. Boshuizen, R. Bromme, H. Gruber (Eds.) Professional Learning: Gaps and transitions on the way from novice to expert (pp. 181-206). Dordrecht, the Netherlands: Kluwer.

Zeichner, K., \& Wray, S. (2001). The teaching portfolio in US teacher education programs: what we know and what we need to know. Teaching and teacher education, 17, 613-621. 


\section{ABOUT THE AUTHOR}

\section{Biography}

In 2008 Simon Beausaert obtained his Masters degree in Educational Sciences at the Catholic University of Leuven. In September 2007 he joined the department Educational Research and Development of the School of Business and Economics, Maastricht University as a junior researcher. Since Augustus 2008 he started his $\mathrm{PhD}$ research project that focuses on the effectiveness of using Personal Development Plans in the workplace. Besides doing research he teaches the course "Schooling and Learning in organizations" and gives trainings in problem based learning. Furthermore he is involved in several consultancy projects on teaching and assessment.

\section{List of publications and presentations}

Beausaert, S., Segers, M., \& Gijselaers, W. (2011). Using a Personal Development Plan for different purposes: Its influence on undertaking learning activities and job performance. Vocations and Learning. Published online.

Beausaert, S., Segers, M., Gijselaers, W. (2011). The Personal Development Plan Practice Questionnaire (PPQ): The development and validation of an instrument to assess employees' perception of the personal development plan practice. International Journal of Training and Development, 15 (5).

Beausaert, S., Segers, M., van der Rijt, J., Gijselaers, W. (2011). The use of Personal Development Plans in the workplace: A literature review. In P. van den Bossche, W. Gijselaers, \& R. Milter (Eds.), Building learning experiences in a changing world, Advances in Business Education and Training III (pp. 235-265). Dordrecht: Springer.

Ketels, N., Beausaert, S., \& Segers, M. (2011). The Use of Personal Development Plans (PDPs) in organizations and the role of its perceived goal. In P. van den Bossche, W. Gijselaers, \& R. Milter (Eds.), Advances in Business Education and Training IV. Dordrecht: Springer.

Rienties, B., Beausaert, S., Grohnert, T., Niemantsverdriet, S., \& Kommers, P. (accepted). Understanding academic performance of international students: The role of ethnicity, academic and social integration.

Van den Bossche, P., \& Beausaert, S. (2011). The reflective practitioner - D. Schön. In (Eds.) F. Dochy, D. Gijbels, M. Segers, \& P. Van den Bossche, Theories of learning for the workplace. Routledge.

Beausaert, S., Sierens, E., Soenens, B., \& Dochy, F. (2009). On the link between perfectionism in teachers and psychological control in teaching: Does burnout play a mediating role? Pedagogische Studiën, 86, 281-295.

Beausaert, S., Segers, M., Fouarge, D., \& Gijselaers, W. (submitted). Is there a difference in undertaking learning activities and performance between pharmacy assistants who are using a Personal Development Plan (PDP) and pharmacy assistants who are not? 
Beausaert, S., Segers, M., \& Gijselaers, W. (accepted with revisions). The use of a Personal Development Plan and the undertaking of learning activities, expertise-growth, flexibility and performance: The role of supporting assessment conditions.

Beausaert, S., Segers, M, Wiltink, D. (submitted). The influence of teachers' teaching approaches on students' learning approaches: The student perspective.

Beausaert, S., Segers, M., \& Gijselaers, W. (2011). The Use of Personal Development Plans (PDPs) in organizations and the role of its perceived goal. Presented at the EARLI 2011, Education for a global networked society, Exeter, United Kingdom, 28 August - 3 September 2011.

Beausaert, S., Segers, M., \& Gijselaers, W. (2011). De lerende werknemer ontPOPt. POPs en de rol van ondersteunende assessment condities. Presented at the Onderwijs Research Dagen (ORD) 2011, Passie voor leren, Maastricht, 8-10 juni 2011.

Beausaert, S., Segers, M., \& Gijselaers, W. (2010). The Personal Development Plan Practice Questionnaire (PPQ): The development and validation of an instrument to assess the employee's perception of the personal development plan practice. Presented at the JURE 2010, Connecting diverse perspectives on learning and instruction, A conference of synergy, Frankfurt am Main, Germany, 19-22 July 2010.

Ketels, N., Beausaert, S., \& Segers, M. (2010). The Use of Personal Development Plans (PDPs) in organizations and the role of it's perceived goal. In S. Halley, C. Birch, D. T. Tempelaar, M. McCuddy, N. Hernández Nanclares, S. Reeb-Gruber, W. H. Gijselaers, B. Rienties \& E. Nelissen (Eds.), 17th EDINEB Conference: Crossing borders in Education and Work-based Learning (pp. 178-186). Maastricht: FEBE ERD Press.

Rienties, B., Beausaert, S., Grohnert, T., Niemantsverdriet, S., Kommers, P., \& Jacobi, R. (2010). The Influence of Academic and Social Integration on Academic Performance for International and Local students, a cross-institutional comparison. In S. Halley, C. Birch, D. T. Tempelaar, M. McCuddy, N. Hernández Nanclares, S. Reeb-Gruber, W. H. Gijselaers, B. Rienties \& E. Nelissen (Eds.), 17th EDINEB Conference: Crossing borders in Education and Work-based Learning (pp. 178-186). Maastricht: FEBE ERD Press.

Beausaert, S., Segers, M., van der Rijt, J., \& Gijselaers, W. (2009). Portfolio-assessment in the workplace: Which goal(s) is it used for, is it effective and which supporting conditions are desirable? A literature review. Presented at the EDINEB conference, Baltimore, United States, 3-5 June 2009. 


\section{SAMENVATTING}

Organisaties maken gebruik van verschillende assessmentvormen die tot doel hebben om de professionele en persoonlijke ontwikkeling van werknemers te promoten (McDowall \& Fletcher, 2004). Voorbeelden van dergelijke assessmentvormen zijn formele functioneringsgesprekken, $360^{\circ}$-feedback en persoonlijke ontwikkelingsplannen (POPs). Over het algemeen kan een POP beschreven worden als een evaluatie-instrument ingebed in een bredere evaluatiecyclus van voortgangs-, functionerings- en beoordelingsgesprekken, bedoeld om informatie te verzamelen over de competenties waaraan de werknemer heeft gewerkt en waaraan de werknemer in de toekomst plant te werken. Het gebruik van een POP wordt aangemoedigd in de hoop dat werknemers intentioneel leeractiviteiten gaan ondernemen en bijgevolg meer kennis verwerven en beter gaan presteren (London, 1997; van de Wiel, Szegedi, \& Weggeman, 2004).

Alhoewel het de trend is om POPs veeleer voor beoordelingsdoeleinden te gebruiken (summatieve assessment), ligt de kracht van het instrument in het ondersteunen van de professionele ontwikkeling van de werknemer (formatieve assessment) (Darling-Hammond \& Snyder, 2000; Smith \& Tillema, 2003). Vragen die centraal staan in het POP zijn immers: Hoe zou jij je competenties evalueren? Wat zijn je sterktes en zwaktes? Hoe wil je je zwaktes aanpakken (welke leeractiviteiten wil je ondernemen)? Heb je je leerdoelen bereikt of wat is de status van je leerdoelen? De vragen zijn bedoeld om de reflectie van de werknemer op zijn/haar competenties te stimuleren. Wanneer een werknemer op zijn/haar sterktes en zwakte reflecteert, wordt hij/zij zich gewaar van de competenties waaraan nog moet gewerkt worden met het oog op het bereiken van een gesteld doel. Om zijn/haar competenties verder te ontwikkelen, gaat de werknemer verscheidene leeractiviteiten ondernemen (bv. het lezen van een boek, iets opzoeken op internet, het raadplegen van een collega, op zoek gaan naar een mentor, het volgen van een training) om vervolgens beter te presteren in zijn/haar job.

In deze samenvatting zullen we eerst voorgaand onderzoek naar POPs bespreken. Vervolgens zullen we de onderzoeksresultaten die in dit boek behandeld werden bespreken en met elkaar in verband brengen. Tot slot zullen we dieper ingaan op de praktische implicaties van dit onderzoek en enkele suggesties doen voor vervolgonderzoek. 


\section{De populariteit voorbij: Op zoek naar empirisch bewijs}

In het verleden werd heel wat geschreven over Persoonlijke ontwikkelingsplannen of portfolio assessment (de laatste term wordt het vaakst teruggevonden in de literatuur). Dit is bewezen in de literatuurstudie (Chapter 2). De literatuurstudie gaf aan dat de zoekterm 'portfolio' waarnaar gezocht werd in tien verschillende databases (in de titel van het artikel) resulteerde in meer dan 3000 hits. Verder resulteerde de zoekterm 'portfolio (in de titel van het artikel) en organisatie' in 580 hits. De meeste literatuur handelt over een schoolse setting (portfolios gebruikt door studenten of leerkrachten) of over de zorgsector (portfolios gebruikt door dokters, verplegers, tandartsen, apothekersassistenten of therapeuten). Daarenboven is de meeste literatuur over portfolios van een beschrijvende aard. Veelal worden cases beschreven waarbij effectief POP-gebruik geïllustreerd wordt. Echter, empirisch bewijs over de effecten van POP-gebruik is beperkt (e.g. Austin, Marini, \& Desroches, 2005; Evans, Ali, Singleton, Nolan, \& Bahrami, 2002; Zeichner \& Wray, 2001). Bij een literatuurstudie over empirisch onderzoek naar het gebruik van POP's werden 54 relevante referenties teruggevonden. Het gebrek aan empirisch onderzoek over dit onderwerp kan verklaard worden door het feit dat POP assessment nog maar sinds het laatste decennium met toenemende mate wordt geïmplementeerd in organisaties. Verder hebben eerdere studies zich vooral geconcentreerd op kleine groepen POP-gebruikers (e.g. Tigelaar, Dolmans, De Grave, Wolfhagen, van der Vleuten, 2006) op basis waarvan resultaten niet kunnen veralgemeend worden naar andere settings. Daarnaast zijn er tot op de dag van vandaag nauwelijks studies over de effecten van POP's die gebruik maken van een controlegroepdesign en kunnen er daardoor geen conclusies worden getrokken over de effectiviteit van POP's. Tot slot zijn de resultaten van de empirische studies tegenstrijdig. De meeste studies geven aan dat POP assessment effectief is voor leren, persoonlijke of professionele ontwikkeling en het verbeteren van de professionele praktijk, terwijl andere studies (e.g. Bunker and Leggett, 2005; Little \& Hayes, 2003) deze positieve bevindingen niet bevestigen. Het wordt beargumenteerd, echter nauwelijks empirisch onderbouwd, dat de effectiviteit van POP assessment afhankelijk is van bepaalde ondersteunende condities, inherent aan de POP praktijk (b.v. begeleiding van een mentor of leidinggevende). Ondanks de populariteit van het instrument bestaat er een niche in de onderzoeksliteratuur naar de effectiviteit van POP's en de noodzakelijke ondersteunende condities die de tool effectief maken. Dit boek wil deze kloof overbruggen en bijdragen aan de optimale inzet van POP's. Daarom werden systematisch drie vragen onderzocht: (1) Is het gebruik van een POP effectief voor het stimuleren van het ondernemen van leeractiviteiten en de prestatie van de werknemer? (2) Voor welke doelen wordt een POP gebruikt en hoe beïnvloedt de perceptie van de werknemer van deze doelen het ondernemen van leeractiviteiten en de prestatie? (3) Beïnvloedt de POP-praktijk, gekenmerkt door drie 
inherente ondersteunende condities (leren en reflecteren van de werknemer, instructie en feedback gegeven door de leidinggevende en de manier waarop de leidinggevende motiveert) het ondernemen van leeractiviteiten, expertisegroei en prestatie? Met andere woorden, eerst vragen we ons af of het gebruik van een POP effectief is. Ten tweede onderzoeken we of de gepercipieerde doelen en ondersteunende condities het effectief gebruik van een POP voor het ondernemen van leeractiviteiten, expertisegroei en prestatie beïnvloeden.

\section{Resultaten: Empirisch bewijs voor effectiviteit, doelen en onder- steunende condities}

In deze paragraaf zullen we een antwoord formuleren op de drie onderzoeksvragen. We zullen de resultaten beargumenteren, gebaseerd op de inzichten verworven aan de hand van de vier empirische studies beschreven in dit boek.

\subsection{Is het gebruiken van een POP effectief voor het ondernemen van leeractivi- teiten en de prestatie van de werknemer?}

Terwijl er wordt verondersteld dat de prestatie van de werknemer zal verbeteren wanneer hij/zij door gebruik te maken van een POP aan zijn/haar competenties werkt, wees de literatuurstudie (Hoofdstuk 2) op tegenstrijdige onderzoeksresultaten. Terwijl vijf studies ook ineffectiviteit aantoonden van POPs voor leren of reflectie en verzamelen van evidentie of documenteren, wezen de overige studies meestal op de positieve effecten van POPs op persoonlijke of voortdurende professionele ontwikkeling ( $\mathrm{N}$ studies $=17$ ), het stimuleren van reflectie ( $\mathrm{N}$ studies $=18$ ) en het verbeteren van de professionele praktijk of prestatie ( $N$ studies $=5)$. De meeste studies maakten gebruik van een kwalitatieve onderzoeksmethode $(N=51)$ of een combinatie van kwantitatieve en kwalitatieve onderzoeksmethoden ( $\mathrm{N}=$ $23)^{22}$. Meer specifiek waren interviews ( $\mathrm{N}$ studies $=27$ ), portfolioanalyses ( $\mathrm{N}$ studies $=16$ ) en focusgroepen ( $N$ studies $=5$ ) de meest gehanteerde kwalitatieve onderzoeksmethoden. Er werd minder gebruik gemaakt van vragenlijsten ( $\mathrm{N}$ studies = 36), alhoewel het de meest gebruikte kwantitatieve methode was.

Daarnaast waren de onderzoeksresultaten van voorgaande studies vaak gebaseerd op kleine samples van participanten (e.g. Tigelaar et al., 2006) op basis waarvan de onderzoeksresultaten moeilijk konden veralgemeend worden. Verder zijn er nauwelijks studies die de effecten van POPs onderzoeken en daarbij gebruik maken van een controlegroepdesign, waardoor de meeste studies geen valide conclusies kunnen trekken over de effectiviteit van POPs. Daarom hebben wij een quasi-

22 De 23 studies werden eveneens meegerekend bij het tellen van de kwalitatieve studies. 
experimentele studie opgezet (Hoofdstuk 3) waarin POP-gebruikers vergeleken worden met niet-gebruikers voor wat betreft het ondernemen van leeractiviteiten het ondernemen van leeractiviteiten in het verleden en het plannen van toekomstige leeractiviteiten - en competentieontwikkeling. De studie was gebaseerd op een grote dataset van apothekersassistenten $(N=2271)$.

De resultaten zijn uiteenlopend. Er werd vastgesteld dat POP-gebruikers in het verleden meer leeractiviteiten ondernamen dan niet-gebruikers. Echter, in tegenstelling tot onze verwachtingen planden apothekersassistenten die gebruik maken van een POP niet meer leeractiviteiten in de toekomst. Ze plannen niet vaker training dan hun collega's die geen gebruik maken van een POP. Deze bevinding kan erop wijzen dat POP's voornamelijk gebruikt worden als feedback tools en niet als feedforward tools. Met andere woorden, we veronderstellen dat POP's vaak gebruikt worden om terug te kijken op ondernomen leeractiviteiten. Ze worden niet gebruikt om vooruit te blikken en om werknemers te ondersteunen in het plannen van toekomstige leeractiviteiten. In vergelijking met andere assessment tools zoals $360^{\circ}$-feedback, die de nadruk legt op het evalueren van voorbije prestaties, incorporeert een POP ook de mogelijkheid tot het vooruit blikken op leren en ontwikkeling (Tillema \& Smith, 2000).

Met betrekking tot de mate waarin apothekersassistenten competent zijn in hun job, hebben we geen significante verschillen gevonden tussen gebruikers en niet-gebruikers van een POP. Dit gebrek aan verschil tussen beide groepen kan verklaard worden doordat apothekersassistenten die gebruik maken van een POP zich beter bewust zijn van de competenties die nodig zijn om hun job te vervullen, net omdat het POP hen stimuleert hierover na te denken. Deze kritische reflectie op competenties door POP-gebruikers kan ervoor zorgen dat zij de eigen competenties gaan onderwaarderen. Als gevolg hiervan kunnen de verschillen tussen beide groepen verdwenen zijn.

Een tweede verklaring is de invloed van het ontbreken aan reflectieskills. Eerder onderzoek heeft aangetoond dat reflectie, een cruciale vaardigheid voor professionele ontwikkeling wanneer gewerkt wordt met een POP, nog steeds een weinig vertrouwde vaardigheid is voor POP-gebruikers (Smith \& Tillema, 1998). Het is mogelijk dat POP gebruikers geen significante verbetering in jobcompetenties vertoonden omwille van het gebrek aan reflectievaardigheden. Bijgevolg is er geen direct verband te maken tussen de zelfreflectie van de werknemer en een verbeterde praktijk (Austin, et al., 2005; Orland-Barak, 2005).

Ten derde kan men zich afvragen in welke mate het gebrek aan positieve effecten van het gebruik van een POP op leren en vervolgens de prestatie contextspecifiek is. Resultaten kunnen verschillen naargelang de leercultuur in de organisatie (Marsick \& Watkins, 2003), sectoren of disciplines (vb. Lee, 2003), type organisatie (marktgeoriënteerd of niet; Baker \& Sinkula, 1999), type werknemer (vb. ervaren versus niet-ervaren) en omvang van de organisatie (kleine en middelgrote bedrij- 
ven versus grote bedrijven). Vaak vertonen grotere organisaties een meer gesofisticeerd human resource management (Guthrie, 2001).

\subsection{Voor welke doelen wordt een POP gebruikt en hoe beïnvloedt de perceptie van de werknemer van deze doelen het ondernemen van leeractiviteiten en de prestatie?}

De literatuurstudie (Hoofdstuk 2) gaf aan dat POP's voor verschillende doeleinden worden gebruikt. Vaak wordt een onderscheid gemaakt tussen professionele ontwikkeling enerzijds en certificatie/selectie/verantwoording anderzijds (Smith \& Tillema, 2001). Wanneer een POP wordt gebruikt voor professionele ontwikkelingsdoeleinden, speelt leren een centrale rol. Indien een POP wordt gehanteerd voor certificatie/selectie/verantwoording, is zichzelf presenteren belangrijker. Bovendien resulteerde de literatuurstudie in de identificatie van negen clusters van doelen. Enerzijds werden de volgende leer- en ontwikkeldoelen geïdentificeerd: (1) professionele ontwikkeling; (2) reflectief leren; (3) coaching; (4) versterken van vertrouwen; en (5) organiseren van leren. Anderzijds werden de volgende certificatie- en selectiedoelen geclusterd: (6) aanvoeren van bewijs; (7) documenteren; (8) certificeren, selecteren en promoten; (9) externe mobiliteit. In Hoofdstuk 4 onderzochten we de invloed van de gepercipieerde doelen op het ondernemen van leeractiviteiten en prestatie bij kenniswerkers van een internationale organisatie die gespecialiseerd is in medische apparatuur en werknemers die werkzaam zijn in een regionale Nederlandse overheidsinstelling. De resultaten gaven aan dat hoewel POP's kunnen gebruikt worden voor het evalueren van jobprestaties, ligt de kracht van het POP in het ondersteunen van werknemers in hun professionele ontwikkeling. Meer specifiek, het gebruik van een POP stimuleert werknemers om te reflecteren op de mate waarin zij de competenties bezitten die noodzakelijk zijn voor het uitvoeren van hun job en op de leeractiviteiten die ze ondernemen om deze competenties aan te scherpen. Daarnaast leidt het gebruik van een POP tot het diagnosticeren van niches in jobcompetenties en stimuleert het bijgevolg werknemers om meer leeractiviteiten te plannen in de toekomst.

Deze resultaten impliceren dat om werknemers leeractiviteiten te laten ondernemen en hun jobprestatie te laten verbeteren door gebruik te maken van een POP, de tool het best als een leer- en ontwikkelinstrument kan geïntroduceerd worden. Daarvoor dient de leidinggevende de leer- en ontwikkeldoelen van het POP expliciet te maken naar de werknemer toe en het gebruik van het leerinstrument te ondersteunen met gepaste begeleiding. Echter, het feit dat ook het percipiëren van het POP als een selectie- en certificatietool het ondernemen van leeractiviteiten en de jobprestatie positief beïnvloedt, leidt tot de vraag: Hoe te balanceren tussen certificatie- en selectiedoelen enerzijds en leer- en ontwikkeldoelen anderzijds, wetende dat de leer- en ontwikkeldoelen sterke voorspellers zijn van 
het ondernemen van leeractiviteiten en prestatie? Brutus, London en Martineau bijvoorbeeld onderzochten $360^{\circ}$-feedback en de relatie ervan met het vooropstellen van ontwikkeldoelen. Uit hun onderzoek bleek dat beoordelingen gegeven door leidinggevenden als minder belangrijk ervaren worden bij het vooropstellen van ontwikkeldoelen dan de feedback gegeven door ondergeschikten en collega's.

\subsection{Beïnvloedt de POP-praktijk, gekenmerkt door drie inherente ondersteunende condities (leren en reflecteren van de werknemer, instructie en feedback gegeven door de leidinggevende en de manier waarop de leidinggevende motiveert) het ondernemen van leeractiviteiten, expertisegroei en prestatie?}

In de studie besproken in Hoofdstuk 6 onderzochten we welke condities de effectiviteit van POP-assessment bevorderen. De literatuurstudie over POP-assessment in organisaties (Hoofdstuk 2) gaf aan dat om de POP-praktijk in organisaties te optimaliseren, er nood is aan verschillende ondersteunende condities. Daarbij wordt een onderscheid gemaakt tussen condities gerelateerd aan hoe het POP geïmplementeerd is in de omgeving (contextuele ondersteunende condities) en condities gerelateerd aan de gebruiker van het POP (individuele ondersteunende condities).

Om de ondersteunende condities inherent aan de POP-praktijk te kunnen meten, hebben we een vragenlijst ontwikkeld en gevalideerd, de POP Praktijk Vragenlijst (PPV). De valideringsstudie (Hoofdstuk 5) werd voltrokken in drie verschillende organisaties: onder werknemers van een internationale organisatie die gespecialiseerd is in logistieke dienstverlening aan bedrijven, experts van een internationale organisatie die gespecialiseerd is in het vervaardigen van medische apparatuur en werknemers van een regionale Nederlandse overheidsinstelling. De studie toonde de constructvaliditeit van de vragenlijst aan die bestond uit drie factoren: Leren en reflecteren ( 8 items, $\alpha=.89$ ), Instructie en feedback ( 5 items, $\alpha=.70$ ) en De motiverende leidinggevende ( 4 items, $\alpha=.74$ ). Ten eerste, de schaal "leren en reflecteren" meet de mate waarin werknemers de leer- en reflectiewaarde van het POP appreciëren. Ten tweede, de instructie- en feedbackschaal vraagt naar de appreciatie van de werknemer ten aanzien van de richtlijnen van de leidinggevende over hoe te werken met het POP en zijn/haar feedback in relatie tot het POP. Er wordt nagegaan of de criteria duidelijk zijn en of de feedback specifiek genoeg en op tijd verkregen is, constructieve suggesties bevat en op regelmatige basis wordt verstrekt. Ten derde, de schaal "motiverende leidinggevende" vraagt naar de perceptie van de werknemer van de mate waarin de leidinggevende voldoende ruimte laat voor initiatief van de werknemer en of de leidinggevende voldoende betrokken is. Beiden hebben een positieve invloed op de motivatie van de werknemer (Deci \& Ryan, 2000).

De drie voorheen genoemde condities onderzoekend, heeft een studie onder werknemers in een overheidsorganisatie (Hoofdstuk 6) aangetoond dat als de 
werknemer reflecteert op het POP en ervan leert en indien de leidinggevende als motiverend wordt gezien, het waarschijnlijker is dat de werknemer leeractiviteiten zal ondernemen, meer expertisegroei en flexibiliteit ten aanzien van veranderende omstandigheden zal vertonen en vervolgens beter zal presteren. Samengevat, redenen voor de effectiviteit van POP's als assessment tool kunnen gevonden worden in de manier waarop het assessmentproces is opgezet en georganiseerd en/of in de aanwezigheid van verschillende ondersteunende condities (leren en reflectie en motiverende leidinggevende). Hoewel we de POP-praktijk onderzocht hebben in organisaties, stemmen onze resultaten overeen met voorgaand onderzoek van Bullock et al. (2007), Tillema (2001) en Tigelaar en collega's (2006b), die de ondersteunende condities onderzochten voor de POP-praktijk in scholen (studenten). In tegenstelling tot wat we verondersteld hadden in onze studie, bleek de instructie en feedback van de leidinggevende geen cruciale rol te spelen. Dit kan verklaard worden door het feit dat veel leidinggevenden het moeilijk vinden om de juiste feedback op de goede manier te geven. Hoe leidinggevenden feedback geven, blijft een hekel punt. Daarnaast staan professionals weigerachtig ten aanzien van feedback van hun leidinggevende (Smith \& Tillema, 2003). Gebruik maken van bijvoorbeeld $360^{\circ}$-feedback, waarbij ook collega's (en klanten) betrokken zijn in het feedbackproces, kan een oplossing zijn voor dit probleem (Brutus et al., 1999).

Verder toonden de resultaten aan dat het effect van het percipiëren van het POP als een leer- en reflectietool op expertisegroei en prestatie sterker is wanneer de werknemer meer leeractiviteiten onderneemt. Verder medieert ook het ondernemen van leeractiviteiten de relatie tussen het percipiëren van een motiverende leidinggevende en de afhankelijke variabelen. Dit illustreert dat het gebruik van een POP als een leer- en reflectietool leidt tot het ondernemen van leeractiviteiten, welke op hun beurt leiden tot expertisegroei en een betere prestatie. Wanneer we deze resultaten samennemen met de resultaten van de studie die de doelen van POP's onderzocht (Hoofdstuk 4), kunnen we concluderen dat wanneer werknemers het POP als een leer- en ontwikkelinstrument percipiëren en de tool ook zo gaan gebruiken, positieve effecten op het ondernemen van leeractiviteiten en prestatie vastgesteld worden. Daarnaast en in lijn met voorgaand onderzoek van London et al. (1999) heeft de begeleiding van een leidinggevende die de werknemer motiveert in het gebruik van een POP een positief effect op het ondernemen van leeractiviteiten en beïnvloedt het bijgevolg de expertisegroei en de prestatie van de werknemer. 


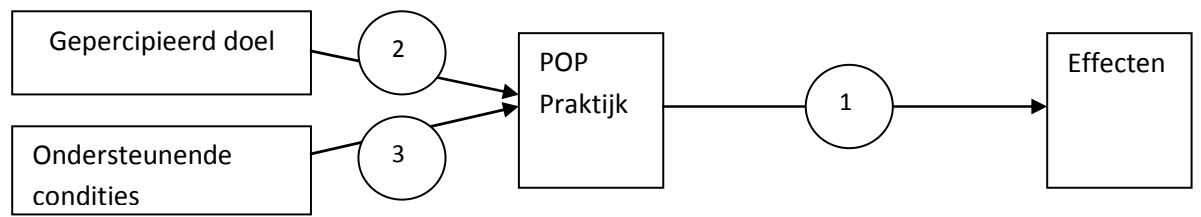

Figuur 1

Overzicht en integratie van de drie centrale onderzoeksvragen.

Samengevat, wanneer we de drie antwoorden op de drie centrale onderzoeksvragen samenbrengen (Figuur 1), kan er geconcludeerd worden dat het gebruik van een POP tot meer ondernomen leeractiviteiten leidt (in het verleden), maar niet aanzet tot het plannen van meer toekomstige leeractiviteiten. Verder lijkt het gebruik van een POP niet effectief om generieke en specifieke jobcompetenties te verbeteren. Echter, om het gebruik van het POP te optimaliseren, dienen het gepercipieerde doel en de ondersteunende condities in rekening te worden gebracht. Het percipiëren van het POP als een leer- en ontwikkelingsinstrument is de sterkste voorspeller van het ondernemen van leeractiviteiten en een betere prestatie, in tegenstelling tot het percipiëren van het POP als een selectie- en certificatietool. Daarnaast kan het hebben van een motiverende leidinggevende die de reflectie door en het leren van de werknemer stimuleert door het geven van duidelijke instructies en feedback, de effecten van het POP-gebruik positief beïnvloeden.

\section{Praktische implicaties voor Human Resource Management en development}

Deze studie heeft enkele implicaties voor human resource management- en development- afdelingen, voor leidinggevenden die werknemers ondersteunen bij het gebruik van een POP en voor werknemers die gebruik maken van de tool. Tabel 1 geeft een overzicht van de praktische implicaties voor de drie partijen met het oog op het zo effectief mogelijk gebruik maken van een POP. De verschillende stappen dienen in volgorde van opsomming doorlopen te worden bij het implementeren van POP-assessment in een organisatie. 
Tabel 1

Te nemen stappen bij het implementeren van een POP

... voor Human Resource 1. Wat is het doel van het Management en Development

$$
\text { gebruik van een POP? }
$$

. Hoe sluit het gebruik maken van een POP aan bij de strategie van de organisatie?

3. Hoe zal het POP geïmplementeerd worden in de organisatie?

4. Hoe zal het POP-formulier er uitzien?

5. Zijn de ondersteunende condities aanwezig?
- Leren en ontwikkelen

- Selecteren en certificeren

- Combinatie van beide doelen; belang van het vinden van een balans tussen beide doelen - Ouder wordende werknemers, innovaties en competitie

- Reorganiseren

- Selecteren van high-potentials

- Voeren van beoordelingsgesprekken

- Wanneer bespreken we het POP?

- Hoe vaak wordt het POP besproken?

- Met wie wordt het POP besproken? (coach versus leidinggevende) - Welke vragen worden gesteld in het POP?

1. Hoe zou jij je competenties evalueren?

2. Wat zijn jouw sterktes en zwaktes?

3. Hoe ga je aan je zwaktes werken (ga je hierbij leeractiviteiten ondernemen en welke)?

4. Heb je je leerdoelen bereikt en/of wat is de status van je leerdoelen?

- Werd het doel, de grondgedachte en de structuur van het POP gecommuniceerd met leidinggevenden en werknemers?

- Is de werknemer aan het reflecteren?

- Krijgt de werknemer de verantwoordelijkheid om het POP in te vullen (autonomie-ondersteuning)? - Is er een leidinggevende of een coach aanwezig die de werknemer begeleidt, motiveert en stimuleert om te reflecteren?

- Zijn de leidinggevenden getraind in het ondersteunen van POPgebruikers?

\footnotetext{
... voor leidinggevenden
}

\section{Introduceer het doel, de} grondgedachte en de structuur van het assessment tool aan de werknemer 


2. Vind een balans tussen
leer- en ontwikkeldoelen
enerzijds en selectie- en
certificatiedoelen anderzijds
3. Gebruik het instrument
niet alleen als feedbacktool,
om terug te kijken op leerac-
tiviteiten, maar ook als
feedforwardtool, om toe-
komstige leeractiviteiten te
plannen. Welke aspiraties
heeft de werknemer en aan
welke competenties dient
hij/zij te werken?
4. Motiveer de werknemer
door zijn/haar reflectie aan
te wakkeren (focus op de
reflectievragen). Help de
werknemer met reflecteren.
1. Neem het initiatief, bereid
de POP-gesprekken voor en
vul het POP-formulier zelf in
2. Gebruik de vragen gesteld
in het POP-formulier om na
te denken over je ontwikke-
ling en toekomstige loop-
baan.

\section{Wat nu?}

Dit onderzoeksproject is een eerste stap in het verzamelen van empirisch bewijs voor de effectiviteit van POP's en de rol van verschillende condities die de effectiviteit ervan bevorderen. Toekomstig onderzoek kan dit project enkele stappen verder brengen.

Ten eerste, het concept POP of portfolio heeft vele connotaties, zowel in de literatuur als in de praktijk. Om de vergelijkbaarheid te bevorderen werden enkel referenties en praktijken die gebruik maken van een POP zoals wij de tool definieerden (Hoofdstuk 2), in rekening gebracht. Toekomstig onderzoek zou niet enkel moeten focussen op de verschillende definities en doelen van een POP, maar ook op het gebruik van verschillende formats. Sommige POP-sjablonen sporen aan tot een diepgaande analyse van de sterktes en zwaktes van de werknemers, bijvoorbeeld, terwijl andere formulieren enkel aandacht hebben voor het definiëren van leerdoelen, hoe de doelen te bereiken en in welk tijdskader. Of terwijl sommige POPs een overzicht geven van de verschillende jobprofielen en de daaraan gerelateerde competenties, is dit bij andere POP-formulieren niet het geval. Verder kan 
de implementatie en procedure bij het gebruik van een POP erg verschillen tussen organisaties. Zo kan bijvoorbeeld het aantal formele gesprekken waarin het POP besproken wordt, variëren. Toekomstig onderzoek zou de effectiviteit van verschillende procedures moeten onderzoeken.

Ten tweede, sectorspecifieke studies zoals de studies beschreven in dit boek hebben als voordeel dat ze focussen op een vrij homogene groep werknemers. Meer bepaald laat het ons toe om te controleren voor sectorale verschillen inzake arbeidsmarktkenmerken (gemiddelde leeftijd van de werknemers, mobiliteit, inzetbaarheid van werknemers, selectie en promotie...). De vier empirische studies in dit boek werden volbracht in vier verschillende organisaties. Hoewel we geen comparatieve studies hebben uitgevoerd, levert het vergelijken van de resultaten van de vier organisaties toch enige indicaties op over de mogelijke verschillen in POP gebruik naargelang het type organisatie. Verschillen tussen de organisaties kunnen als volgt worden verklaard (Tabel 2 ).

Tabel 2

Vergelijking van de karakteristieken van de organisaties betrokken in het onderzoeksproject

\begin{tabular}{|c|c|c|c|}
\hline $\begin{array}{l}\text { Organisatie 1: Apo- } \\
\text { thekerassistenten } \\
\text { (Hoofdstuk 3) }\end{array}$ & $\begin{array}{l}\text { Organisatie 2: Gou- } \\
\text { vernementele organi- } \\
\text { satie (Hoofdstuk 4, } 5 \\
\text { en 6) }\end{array}$ & $\begin{array}{l}\text { Organisatie 3: Medi- } \\
\text { sche apparatuur } \\
\text { (Hoofdstuk } 4 \text { en 5) }\end{array}$ & $\begin{array}{l}\text { Organisatie 4: Logis- } \\
\text { tieke bedrijfsverlening } \\
\text { (Hoofdstuk 5) }\end{array}$ \\
\hline Profit & Non-profit & Profit & Profit \\
\hline Klein & Groot & Groot & Groot \\
\hline $\begin{array}{l}\text { Weinig carrièrepers- } \\
\text { pectieven }\end{array}$ & $\begin{array}{l}\text { Geen carrièreperspec- } \\
\text { tieven }\end{array}$ & Carrièreperspectieven & Carrièreperspectieven \\
\hline$?$ & $\begin{array}{l}\text { Niet goed geïmple- } \\
\text { menteerd in het } \\
\text { assessment-systeem } \\
\text { (recent geherstructu- } \\
\text { reerd) }\end{array}$ & $\begin{array}{l}\text { Goed geïmplemen- } \\
\text { teerd in het asses- } \\
\text { sment-systeem ( } 5 \text { jaar) }\end{array}$ & $\begin{array}{l}\text { Goed geïmplemen- } \\
\text { teerd in het asses- } \\
\text { sment-systeem ( } 5 \text { jaar) }\end{array}$ \\
\hline$M_{\text {leeftijd }}=39.54$ & $M_{\text {leeftijd }}=49$ & $M_{\text {leeftijd }}=35-41$ & $M_{\text {leeftijd }}=36-40$ \\
\hline
\end{tabular}

Ten eerste, Organisatie 2 is een non-profitorganisatie. Dit betekent dat de organisatie geen competitie hoeft te voeren met andere bedrijven en daarom niet gedwongen wordt om mee te zijn met de nieuwste ontwikkelingen. Leren en ontwikkeling speelt niet altijd een centrale rol in de verschillende departementen in Organisatie 2. De andere organisaties daarentegen zijn profitorganisaties. Dit betekent dat deze organisaties meer dynamische organisaties zijn die steeds moeten meegroeien met de nieuwste ontwikkelingen om zo te kunnen concurreren met andere bedrijven. Bijgevolg is leren en ontwikkelen deel van de organisatiecultuur en altijd tegenwoordig.

Ten tweede, de werknemers van Organisatie 1 zijn werkzaam in verschillende kleine organisaties. In kleine organisaties is de nood aan formele assessmentsystemen die de continue professionele ontwikkeling van werknemers structureren en 
ondersteunen minder groot, omdat de werknemer een nauw contact heeft met de leidinggevende die in principe het formele controlesysteem vervangt. Bijgevolg wordt de voorkeur gegeven aan informeel leren (Saru, 2007) en POP's, die vaak focussen op formeel leren, worden daardoor niet altijd gepercipieerd als noodzakelijk en effectief. Organisatie 2, 3 en 4 zijn grote organisaties waar een meer formeel assessmentsysteem noodzakelijk is om de ontwikkeling van de werknemers te structureren. Echter, POP's lijken niet het effect te hebben dat ze verondersteld worden te hebben. Of ondersteunende condities al dan niet aanwezig zijn, kan alvast gedeeltelijk de verschillen tussen een effectief en een minder effectief gebruik van POP's verklaren. Ten derde, gouvernementele organisaties (Organisatie 2) zijn meer stabiele en bureaucratische organisaties waarbij de kennis en vaardigheden die werknemers nodig hebben minder dynamisch zijn. Loopbaanperspectieven zijn ook beperkt, wat betekent dat competitie tussen werknemers beperkt is en kennis niet wordt gezien als een competitief voordeel. Organisatie 1 en 3 situeren zich in de farmaceutische en medische sector en Organisatie 4 situeert zich in de logistieke dienstverleningssector. Het betreft sectoren waar kennis sterkt evolueert (hoge industriedynamiek; Datta, Guthrie, \& Wright, 2005). Verder bieden ze meer carrièreperspectieven waarbij leren en het hebben van expertise gepercipieerd wordt als een competitief voordeel. In Organisatie 3 bijvoorbeeld percipiëren werknemers het POP voornamelijk als een certificatie- en selectietool en werknemers ondernemen er dan ook significant meer leeractiviteiten dan de werknemers in Organisatie 2 (Hoofdstuk 4). Daarenboven maakt het POP in Organisatie 3 deel uit van een beter geïmplementeerd assessmentsysteem waarbij het al dan niet gebruiken van de tool bepalend kan zijn voor het al dan niet krijgen van een promotie. Ten vierde, de gemiddelde leeftijd van de werknemer in Organisatie 2 is vandaag 49 jaar. Dit betekent dat een grote groep werknemers kan beschouwd worden als experts die niet langer geïnteresseerd zijn in systematisch leren en ontwikkelen (Cleveland, \& Shore, 1992). Organisatie 1, 3 en 4 beschikken over een jongere staf; de gemiddelde leeftijd ligt tussen 35 en 41 jaar. Het is waarschijnlijk dat een grotere groep beginners geïnteresseerd is in leren en ontwikkelen en bijgevolg promotie maken en/of hun inzetbaarheid vergroten. Deze voorlopige resultaten en suggesties dienen ter validering gereproduceerd te worden in andere sectoren. Toekomstig onderzoek zou de effectiviteit van POP's voor het ondernemen van leeractiviteiten en een betere prestatie moeten onderzoeken bij een bredere sample participanten die verschillen wat betreft beroep, land van afkomst, type contract, teams, werkschema's, expertiseniveaus en inzetbaarheid. Verder kunnen effecten en ondersteunende condities verschillen naargelang de leercultuur in een organisatie (Marsick \& Watkins, 2003), sectoren of disciplines (e.g. Datta, Guthrie, \& Wright, 2005), type organisatie (marktgeoriënteerd of niet; Baker \& Sinkula, 1999), type werknemer (vb. ervaren versus niet ervaren) en organisatiegrootte 
(klein versus groot bedrijf; Saru, 2007). Vaak vertonen grotere organisaties een meer gesofisticeerd human resource management (Guthrie, 2001).

Ten derde, er werd aangetoond dat een POP van hoge kwaliteit enkel mag verwacht worden wanneer het al voor een langere periode wordt gebruikt (Smith \& Tillema, 1998). De organisaties die in dit boek aan de orde waren startten op verschillende momenten met het gebruiken van POP's. Organisatie 2 gebruikt de tool al enkele jaren en kwam recent met een geüpdate format voor de dag. Organisatie 3 en 4 gebruiken het POP al vijf jaar. In lijn met het onderzoek van Smith en Tillema (1998) werd vastgesteld dat werknemers in Organisatie 3 significant meer leeractiviteiten ondernemen dan de werknemers in Organisatie 2. Gelijkaardige studies met een longitudinaal onderzoeksdesign zouden de effectiviteit van POP's op de werkplek meer accuraat kunnen meten. Dit onderzoeksdesign zou ons ook toelaten om conclusies te trekken over causale relaties.

Ten vierde, aangezien werd aangetoond dat assessmentpraktijken de leerresultaten beïnvloeden via de perceptie van de werknemer (Biggs, 2003), geloven we dat het meten van de perceptie van de werknemer de beste manier is om effecten van POP-assessment te meten. Echter, voor wie geïnteresseerd zou zijn in het onderzoeken van het gedrag van werknemers bij het gebruik van een POP, het leren ervan en de verbeterde prestatie, is het aan te raden gebruik te maken van meerdere beoordelaars en bijvoorbeeld ook leidinggevenden te bevragen.

Afsluitend, het is niet het instrument op zich dat werknemers laat leren en ontwikkelen, het is de manier waarop het geïmplementeerd is en hoe het gebruikt wordt dat bepalend is voor het al dan niet slagen van een assessmentpraktijk, los van welk assessment tool gebruikt wordt. Onderzoek zou zich moeten focussen op de assessmentpraktijk en de ondersteunende condities binnen die praktijk. Assessment tools zoals POP's zouden vooral gebruikt moeten worden als leer- en ontwikkel-instrumenten, aangezien daar hun kracht ligt. 


\section{Referenties}

Austin, Z., Marini, A., \& Desroches, B. (2005). Use of a learning portfolio for continuous professional development: A study of pharmacists in Ontario (Canada). Pharmacy Education, 5, 175-181.

Baker, W.E., \& Sinkula, J.M. (1999). The synergistic effect of market orientation and learning orientation on organizational performance. Journal of the Academy of Marketing Science, 27, 411-427.

Biggs, J. (2003). Teaching for quality learning at university. Maidenhead: McGraw-Hill Education.

Brutus, S., London, M., \& Martineau, J. (1999). The impact of 360-degree feedback on planning for career development. Journal of Management Development, 18, 676-693.

Bullock, A., Firmstone, V., Frame, J., \& Bedward, J. (2007). Enhancing the benefit of continuing professional development: A randomized controlled study of personal development plans for dentists. Learning in Health and Social Care, 6 (1), 14-26.

Bunker, A. \& Leggett, M. (2005). Teaching portfolios: rhetoric, reality and reflection (unpublished).

Cleveland, J., \& Shore, L. (1992). Self- and supervisory perspectives on age and work attitudes and performance. Journal of Applied Psychology, 77, 469-484.

Darling-Hammond, L., \& Snyder, J. (2000). Authentic assessment of teaching in context. Teaching \& Teacher Education, 16, 523-545.

Datta, D.K., Guthrie, J.P., \& Wright P.M. (2005). Human Resource Management and labor productivity: Does industry matter? Academy of Management Journal, 48, 135-145.

Deci, E. L. \& Ryan, R. M. (2000). The "What" and "Why" of Goal Pursuits: Human Needs and the SelfDetermination of Behavior. Psychological Inquiry: An International Journal for the Advancement of Psychological Theory, 11 (4), 227-268.

Evans, A., Ali, S., Singleton, C., Nolan, P., \& Bahrami, J. (2002). The effectiveness of personal education plans in CPD: An evaluation. Medical Teacher, 24 (1), 79-84.

Guthrie, J.P. (2001). High-Involvement Work Practices, Turnover, and Productivity: Evidence from New Zealand. The Academy of Management Journal, 44, 180-190.

Lee, C.H., \& Bruvold, N.T. (2003). Creating Value for Employees: Investment in Employee Development. International Journal of Human Resource Management, 14, 981-1000.

Little, P., \& Hayes, S. (2003). Continuing professional development (CPD): GPs' perceptions of postgraduate education-approved (PGEA) meetings and personal professional development plans (PDPs). Family Practice, 20 (2), 192-198.

London, M. (1997). Job feedback: Giving, seeking, and using feedback for performance improvement. Mahwah, NJ: Erlbaum.

London, M., Larsen, H.H., \& Thisted, L.N. (1999). Relationships between feedback and self-development. Group \& Organizational Management, 24 (1), 5-27.

Marsick, V.J. \& Watkins, K.E. (2003). Demonstrating the value of an organization's learning culture: The dimensions of the learning organization questionnaire. Advances in Developing Human Resources, 5, 132-151.

McDowall, A., \& Fletcher, C. (2004). Employee development: An organizational justice perspective. Personnel Review, 33: 8-29.

Orland-Barak, L. (2005). Portfolios as evidence of reflective practice: What remains "untold". Educational Research, 47 (1), 25-44.

Saru, E. (2007). Organisational learning and HRD: how appropriate are they for small firms? Journal of European Industrial Training, 31 (1), $36-51$.

Smith, K., \& Tillema, H. (1998). Evaluating portfolio use as a learning tool for professionals. Scandinavian Journal of Educational Research, 42, 193-205.

Smith, K., \& Tillema, H. (2001). Long-term influences of portfolios on professional development. Scandinavian Journal of Educational Research, 45, 183-202.

Smith, K., \& Tillema, H. (2003). Clarifying different types of portfolio use. Assessment \& Evaluation in Higher Education, 28, 625-648. 
Tigelaar, D.E.H., Dolmans, D.H.J.M., de Grave, W.S., Wolfhagen, I.H.A.P., van der Vleuten, C.P.M. (2006). Participants' opinions on the usefulness of a teaching portfolio. Medical Education, 40, 371-378.

Tigelaar, D.E.H., Dolmans, D.H.J.M., De Grave, W.S., Wolfhagen, I.H.A.P., \& Van der Vleuten, C.P.M. (2006b). Portfolio as a tool to stimulate teachers' reflections. Medical Teacher, 28, 277-282.

Tillema, H.H., \& Smith, K. (2000). Learning from portfolios: Differential use of feedback in portfolio construction. Studies in Educational Evaluation, 26, 193-210.

Tillema, H.H. (2001). Portfolios as developmental assessment tools. International Journal of Training and Development, 5 (2), 126-135.

Van de Wiel, M.W.J., Szegedi, K.H.P., \& Weggeman, M.C.D.P. (2004). Professional learning: Deliberate attempts at developing expertise. In: H.P.A. Boshuizen, R. Bromme, H. Gruber (Eds.) Professional Learning: Gaps and transitions on the way from novice to expert (pp. 181-206). Dordrecht, the Netherlands: Kluwer.

Zeichner, K., \& Wray, S. (2001). The teaching portfolio in US teacher education programs: what we know and what we need to know. Teaching and teacher education, 17, 613-621. 



\section{ICO DISSERTATION SERIES}

In the ICO Dissertation Series dissertations are published of graduate students from faculties and institutes on educational research within the following universities: Eindhoven University of Technology, Leiden University, Maastricht University, Open University of the Netherlands, University of Amsterdam, University of Groningen, University of Twente, Utrecht University, VU University Amsterdam, and Wageningen University (and formerly Radboud University Nijmegen and Tilburg University). Below is a list of the most recent dissertations:

Munneke-de Vries, E.L. (11-01-2008). Arguing to learn: Supporting interactive argumentation through computer-supported collaborative learning. Utrecht: Utrecht University.

Nijveldt, M.J. (16-01-2008). Validity in teacher assessment. An exploration of the judgement processes of assessors. Leiden: Leiden University.

Jonker, H.G. (14-02-2008). Concrete elaboration during knowledge acquisition. Amsterdam: VU University Amsterdam.

Schuitema, J.A. (14-02-2008). Talking about values. A dialogue approach to citizenship education as an integral part of history classes. Amsterdam: University of Amsterdam.

Janssen, J.J.H.M. (14-03-2008). Using visualizations to support collaboration and coordination during computer-supported collaborative learning. Utrecht: Utrecht University.

Honingh, M.E. (17-04-2008). Beroepsonderwijs tussen publiek en privaat: Een studie naar opvattingen en gedrag van docenten en middenmanagers in bekostigde en niet-bekostigde onderwijsinstellingen in het middelbaar beroepsonderwijs. Amsterdam: University of Amsterdam.

Baartman, L.K.J. (24-04-2008). Assessing the assessment: Development and use of quality criteria for competence assessment programmes. Utrecht: Utrecht University.

Corbalan Perez, G. (25-04-2008). Shared control over task selection: Helping students to select their own learning tasks. Heerlen: Open University of the Netherlands.

Hendrikse, H.P. (22-05-2008). Wiskundig actief: Het ondersteunen van onderzoekend leren in het wiskunde onderwijs. Enschede: University of Twente.

Moonen, M.L.I. (26-09-2008). Testing the multi-feature hypothesis: Tasks, mental actions and second language acquisition. Utrecht: Utrecht University.

Hooreman, R.W. (18-11-2008). Synchronous coaching of the trainee teacher: An experimental approach. Eindhoven: Eindhoven University of Technology.

Bakker, M.E.J. (02-12-2008). Design and evaluation of video portfolios: Reliability, generalizability, and validity of an authentic performance assessment for teachers. Leiden: Leiden University.

Kicken, W. (12-12-2008). Portfolio use in vocational education: Helping students to direct their learning. Heerlen: Open University of the Netherlands.

Kollöffel, B.J. (18-12-2008). Getting the picture: The role of external representations in simulation-based inquiry learning. Enschede: University of Twente.

Walraven, A. (19-12-2008). Becoming a critical websearcher: Effects of instruction to foster transfer. Heerlen: Open University of the Netherlands.

Radstake, H. (14-05-2009). Teaching in diversity: Teachers and pupils about tense situations in ethnically heterogeneous classes. Amsterdam: University of Amsterdam. 
Du Chatenier, E. (09-09-2009). Open innovation competence: Towards a competence profile for interorganizational collaboration in innovation teams. Wageningen: Wageningen University.

Van Borkulo, S.P. (26-06-2009). The assessment of learning outcomes of computer modelling in secondary science education. Enschede: University of Twente.

Handelzalts, A. (17-09-2009). Collaborative curriculum development in teacher design teams. Enschede: University of Twente.

Nievelstein, F.E.R.M. (18-09-2009). Learning law: Expertise differences and the effect of instructional support. Heerlen: Open University of the Netherlands.

Visser-Wijnveen, G.J. (23-09-2009). The research-teaching nexus in the humanities: Variations among academics. Leiden: Leiden University.

Van der Rijst, R.M. (23-09-2009). The research-teaching nexus in the sciences: Scientific research dispositions and teaching practice. Leiden: Leiden University.

Mainhard, M.T. (25-09-2009). Time consistency in teacher-class relationships. Utrecht: Utrecht University.

Van Ewijk, R. (20-10-2009). Empirical essays on education and health. Amsterdam: University of Amsterdam.

Seezink, A. (18-11-2009). Continuing teacher development for competence-based teaching. Tilburg: Tilburg University.

Rohaan, E.J. (09-12-2009). Testing teacher knowledge for technology teaching in primary schools. Eindhoven: Eindhoven University of Technology.

Kirschner, F.C. (11-12-2009). United brains for complex learning. Heerlen: Open University of the $\mathrm{Ne}-$ therlands.

Wetzels, S.A.J. (18-12-2009). Individualized strategies for prior knowledge activation. Heerlen: Open University of the Netherlands. 